\title{
Phenolic compounds in agri-food by-products, their bioavailability and health effects
}

\author{
Fereidoon Shahidi*, Varatharajan Vamadevan ${ }^{\dagger}$, Won Young Oh and Han Peng
}

\begin{abstract}
Department of Biochemistry, Memorial University of Newfoundland, St. John's, NL, A1B 3X9, Canada
†Present address: Cargill, 14800 28th Avenue N., Plymouth, MN 55447, USA.

*Corresponding author: Fereidoon Shahidi, Department of Biochemistry, Memorial University of Newfoundland, St. John's, NL, A1B 3X9, Canada. Tel: 709-864-8552; Fax: 709-864-2422; E-mail: fshahidi@mun.ca
\end{abstract}

DOI: $10.31665 / J F B .2019 .5178$

Received: February 01, 2019; Revised received \& accepted: March 01, 2019

Citation: Shahidi, F., Vamadevan, V., Oh, W.Y., and Peng, H. (2019). Phenolic compounds in agri-food by-products, their bioavailability and health effects. J. Food Bioact. 5: 57-119.

\begin{abstract}
Phenolic compounds constitute a large and diverse group of secondary metabolites derived from phenylalanine and tyrosine and are widely distributed throughout the plant kingdom. They could be divided into different classes such as simple phenol, phenyl acetic acid, hydroxybenzoic acid, hydroxycinnamic acid, and other phenylpropanoids as well as condensed tannins (proanthocyanidins) and hydrolysable tannins, among others, depending on their basic carbon skeleton structure. Phenolic compounds in plant-based foods have been suggested to have a number of beneficial health effects including prevention of cancer, cardiovascular disease, diabetes, immune disorders, neurogenerative disease and others. These properties are largely attributable to the antioxidant activity of the phenolic compounds as well as other mechanisms of action. Therefore, nutraceuticals of plant origin may evolve to be considered a vital aspect of dietary-disease preventive food components. Agri-food industries generate substantial quantities of phenolic rich by-products, which could serve as an attractive and commercially viable source of nutraceuticals. This contribution mainly summarizes the occurrence of phenolic compounds and some other bioactives in various Agri-food by-products, their bioavailability and health benefits.
\end{abstract}

Keywords: By-products; Bioactive compounds; Phenolics; Nutraceuticals; Health benefits; Bioavailability.

\section{Introduction}

Plants produce a great variety of organic compounds that comprise both major types of chemicals such as carbohydrate, protein, lipid and nucleic acids as well as non-nutritive phytochemicals. Though phytochemicals, by the strictest definition, are chemicals that are produced by the plants, currently the term is being only used for non-nutritive (not considered as essential nutrients), biologically active, chemically derived compounds found in plants (Alasalvar and Shahidi, 2009). Many of the phytochemicals have the capacity to alter enzymatic and chemical reactions, and therefore may impact human health both positively and negatively (Thompson, 1993).

Most phytochemicals can be classified into three major groups of terpenoids, phenolic compounds, and alkaloids as well as other nitrogen containing plant constituents (Harborn, 1999). Apart from this classification, the main bioactive phytochemicals in foods include polyphenols, terpenoids, glucosinolates and other sulphurcontaining compounds (Espín et al., 2007; Tomás-Barberán et al., 2004). Phenolic compounds constitute a very large group of phytochemicals that are widely distributed in higher plants (Macheix et al., 1990). The general break down of non-nutritive plant-base natural products is shown in Figure 1.

Phenolics are secondary metabolites synthesized by plants both during normal development (Harborne, 1982; Pridham, 1960; Shahidi and Naczk, 2004, Shahidi and Peng, 2018) and in response to stress conditions such as infection by pathogens and parasites, wounding, air pollution, exposure to extreme temperatures and UV 


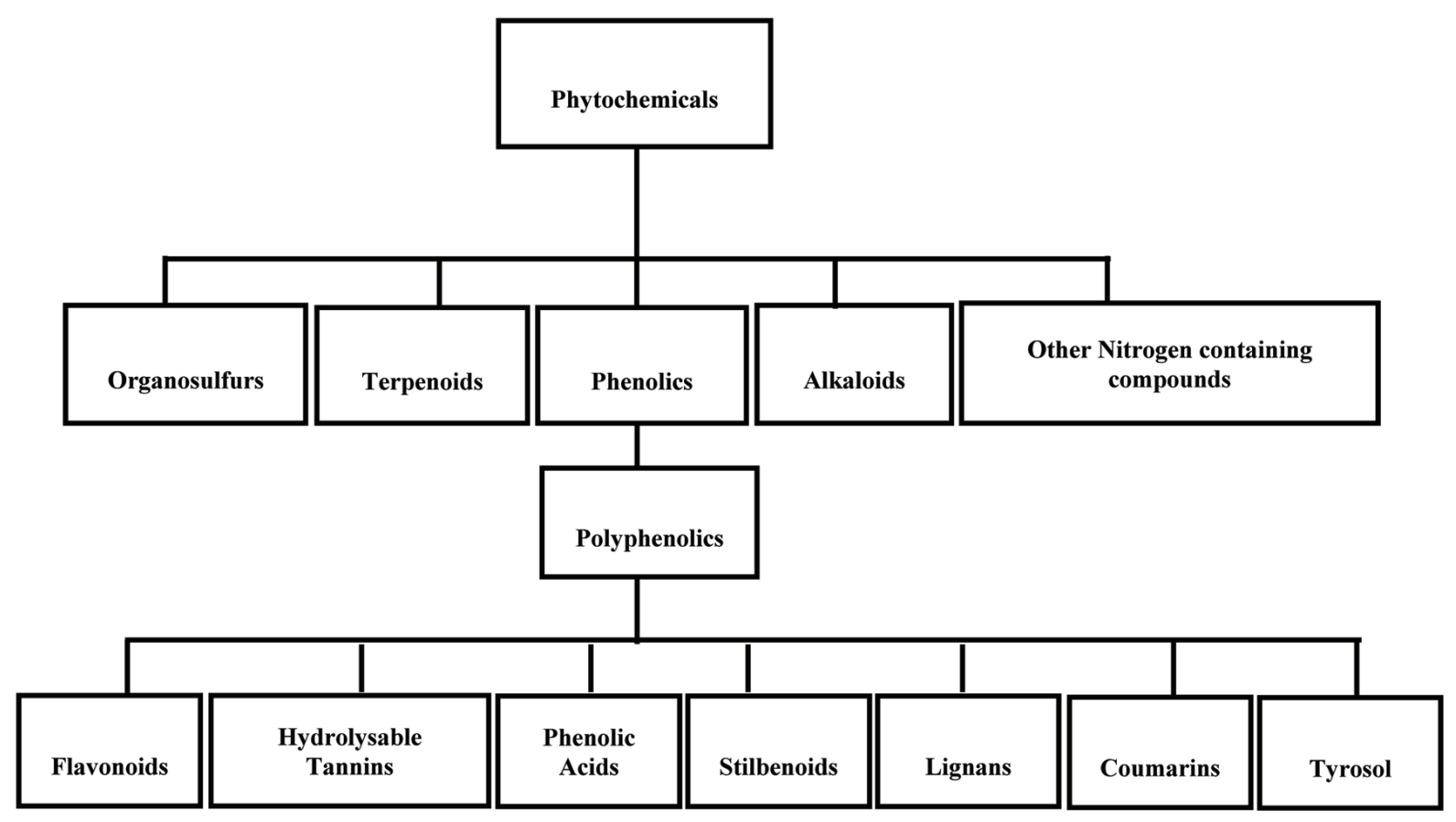

Figure 1. General breakdown of non-nutritive plant-base natural products.

radiation (Beckman, 2000; Nicholson and Hammerschmidt, 1992; Zobel 1997; Shahidi and Yeo, 2016). They are commonly found in both edible and non-edible plant parts and may act as phytoalexins, antifeedants, attractants for pollinators, contributors to plant pigmentation, antioxidants and protective agents against UV light. In addition, phenolics may contribute to the bitterness, astringency, color, flavor, odour, and oxidative stability of food (Shahidi and Naczk, 2004).

Phenolic compounds exhibit a wide range of physiological properties, such as anti-allergenic, anti-artherogenic, anti-inflammatory, anti-microbial, antioxidant, anti-thrombotic, cardioprotective and vasodilatory effects (Benavente-Garcia et al., 1997; Manach et al., 2005; Middleton et al., 2000; Puupponen-Pimiä et al., 2001; Samman et al., 1998, Shahidi and Peng, 2018). Therefore, consumption of plant-based foods is instrumental in health promotion and disease risk reduction. Furthermore, consumers are increasingly aware of diet related health problems, therefore demanding natural ingredients, which are expected to be safe and health promoting (Schieber et al., 2001b).

The agri-food industry produces large volumes of wastes, both solids and liquid, resulting from the production, preparation and consumption of food. In addition to food wastes, food industry uses a large amount of water and proportion of water used may leave as part of the products (blanching water, olive mill wastewater, etc.). In many cases, by-products from agri-food industry are being increasingly recognized as a good and inexpensive source for obtaining bioactive compounds including high-phenolic products (Peng et al., 2018; Shahidi, 2009; Schieber et al., 2001b; Tomás-Barberán et al., 2004). Thus, a number of studies to examine the occurrence of phenolic compounds in various by-products/ residuals of agri-food industry and their antioxidant activity have increased considerably in recent years and the major/selected byproducts of processing industry have been summarized and reviewed as a source of antioxidant (Balasundram et al., 2006; Dimitrios, 2006; Moure et al., 2001; Schieber et al., 2001b; Shahidi,
2008; Peng et al., 2018).

This contribution aims to provide an overview of the findings related to the phenolic profiles of various by-products, their bioavailability and health benefits. Other nutraceuticals in by-products particularly referred to carotenoids and betalains also have been reviewed in this work, but to a lesser extent.

\section{Dietary phenolics and nutraceuticals: overview}

Phenolics are a group of organic compounds with one or more hydroxyl groups on the aromatic ring(s). They range from simple phenols, which are relatively rare (either absent or comprise only a small proportion) in their natural distribution (Cowan, 1999) to complex compounds known as polyphenols (Bravo 1998). These diversified groups of phytochemicals are derived from phenylalanine and tyrosine (Harborne, 1982; Morello et al., 2002; Shahidi, 2000; Shahidi and Naczk, 2004; Shahidi and Yeo, 2018; Shahidi and Peng, 2018). Most naturally occurring phenolic compounds are present as conjugates with mono- and polysaccharides, linked to one or more of the phenolic groups and may also occur as functional derivatives such as methyl and other esters (Harborne, 1989; Harborne et al., 1999; Shahidi and Naczk, 1995). Flavonoids, phenolic acids, and tannins are the most important dietary phenolics (King and Young, 1999).

\subsection{Flavonoids}

The largest group of polyphenols is the flavonoids, which account for $60 \%$ of the total dietary phenolic compounds (Harborne and Williams, 2000; Nichenametla et al., 2006; Shahidi and Naczk, 2004) and there are an estimated 4,000 known flavonoids (Wrolstad, 2005). Flavonoids are $\mathrm{C}_{15}$ compounds all of which have the structure C6-C3-C6 (Harborne and Simmonds, 1964). They gener- 


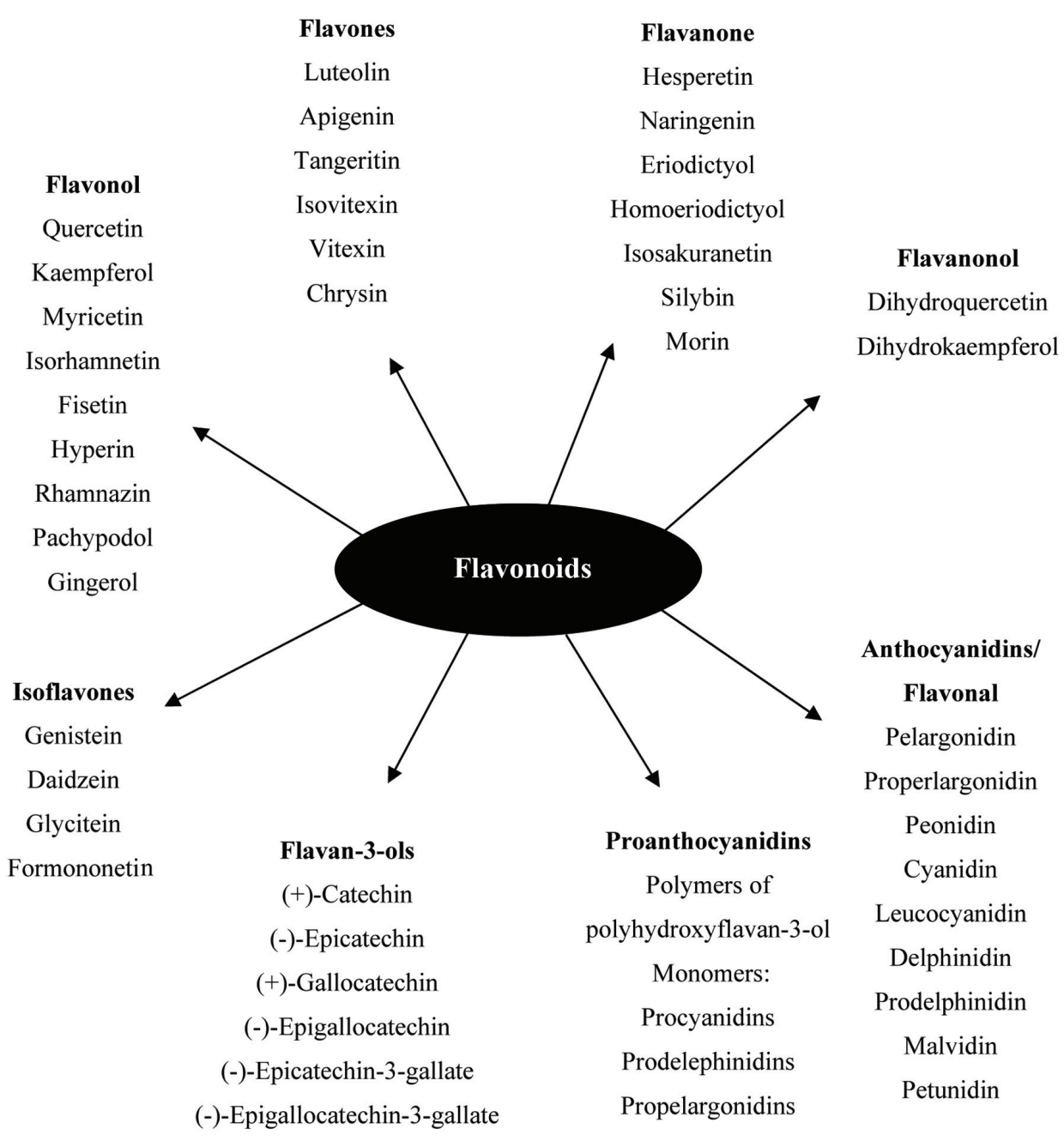

Figure 2. Major classes of flavonoids and its some individual compounds.

ally consist of two aromatic rings, each containing at least one hydroxyl, which are connected through a three-carbon "bridge" and become part of a six-member heterocyclic ring.

The flavonoids are further divided into subclasses based on the connection of an aromatic ring to the heterocyclic ring, as well as the oxidation state and functional groups of the heterocyclic ring. Within each subclass, individual compounds are characterized by specific hydroxylation and conjugation patterns (Merken and Beecher, 2000). Figures 2 and 3 show the major classes of flavonoids and their generic structures, respectively. Among the major classes of flavonoids, flavones and flavonols are the most widely occurring and structurally diverse compounds (Harborne et al., 1999).

Flavonoids occasionally occur in plants as aglycones although they most commonly present as glycoside derivatives. Each hydroxyl group and certain carbon atoms in their structure can be substituted with one or more of a range of different simple carbohydrates which, in turn, may be acylated with a variety of phenolic or aliphatic acids (Harborne and Williams, 2000; Williams and Grayer, 2004). Flavonoids are most commonly known for their antioxidant activity. However, it is now known that the health benefits they provide against cancer and heart disease are the result of other mechanisms (Marais et al., 2006; Shahidi and Yeo, 2018).
Apart from various vegetables and fruits, flavonoids are found in seeds, nuts, grains, spices, and different medicinal plants as well in beverages, such as wine, tea and beer (Shahidi and Naczk, 1995; Yeo and Shahidi, 2017).

\subsection{Phenolic acids}

Phenolic acids, in the form of substituted derivatives of hydroxybenzoic and hydroxycinnamic acids, are the predominant phenolics in food from plant sources, manly grains, legumes and oilseeds. These derivatives differ in the pattern of their hydroxylation and methoxylation on the aromatic ring(s).

Hydroxybenzoic acids (Figure 4) such as gallic acid, $p$-hydroxybenzoic acid and vanillic acid are present in nearly all plants (Robbins, 2003; Shahidi and Naczk, 1995), but foods from plant sources are generally low in hydroxybenzoic acids (Ssonko and Xia, 2005). They are mainly found in the bound state in food and are components of complex structures like hydrolysable tannins and lignin. Aldehydes such as vanillin and $p$-hydroxybenzaldehyde are common flavor compounds derived from the reduction of hydroxybenzoic acids (Shahidi and Naczk, 1995; Shahidi and Yeo, 2016). Dimeric derivative of gallic acid is called ellagic acid 
<smiles></smiles><smiles>O=C1CC(c2ccccc2)Oc2ccccc21</smiles>

Flavanone<smiles>OC1Cc2ccccc2OC1c1ccccc1</smiles>

Flavan-3-ol<smiles>O=C1c2ccccc2OC(c2ccccc2)C1O</smiles>

Flavanonol<smiles></smiles>

Anthocyanidin

Figure 3. Generic structures of major classes of flavonoids.

(Figure 5), which mainly exists in higher plants, including fruits and nuts, combined with its precursor, hexahydroxydiphenic acid or bound in the form of ellagitannins (Amakura et al., 2000). Extracts from red raspberry leaves or seeds, pomegranates are said to contain high levels of ellagic acid (Espín et al., 2007). Gallic acid and ellagic acid (found as hydrolysable tannin) were found as major phenolic compounds in pomegranate peels (Ambigaipalan et al., 2016).

The hydroxycinnamic acids (caffeic, ferulic, sinapic, $p$-coumaric and chlorogenic acid) (Figure 6) are the most widely occurring phenylpropanoids and are precursors to their cyclic derivatives, the coumarins. They are rarely encountered in the free state in nature and are predominantly found as hydroxyacid esters with quinic, shikimic or tartaric acid (Herrmann, 1989), with larger phenolic compounds such as flavonoids or with structural components of the plant such as cellulose, lignin and protein (Clifford, 1999; Kroon and Williamson, 1999; Scalbert and Williamson, 2000). Caffeic acid and, to a lesser extent, ferulic acid are the most prominent phenolic acids occurring in foods of plant origin such as cereals, coffee, fruits and vegetables (Andreasen et al., 2000; Robbins, 2003; Scalbert and Williamson, 2000). Chlorogenic acids (Figure 7) are a family of esters formed between certain trans-cinnamic acids (caffeic acid) and (-)-quinic acid (Clifford, 1999, 2000; Clifford et al., 2003; IUPAC, 1976). Coffee beans are one of the richest dietary sources of chlorogenic acids (Clifford, 1999).

\subsection{Tannins}

Tannins can range from dimers through large polymers and are found in a wide variety of foods, e.g., apples, berries, chocolate, red wines, pomogranate and nuts (Shahidi and Naczk, 2004; de Camargo et al., 2014a; Ambigaipalan et al., 2016). They are polymerized into large molecules, either by the plants themselves or as a result of food processing. Important subclasses of tannins in food include condensed tannins or proanthocyanidins, derived tannins and hydrolysable tannins (Clifford, 2001). Derived tannins (theaflavin and thearubigin) are formed during food handling and processing, and found primarily in black and oolong teas and may be important in the health related properties of these foods (Hakim et al., 2003; Kris-Etherton, and Keen, 2002; Riemersma et al., 2001) (Figure 8).

Hydrolysable tannins are esters of gallic or ellagic acid (gallotannins and ellagitannins) while the proanthocyanidins are polymers of polyhydroxyflavan-3-ol monomers, linked through carbon-carbon and ether linkages (Porter, 1989). Although hydrolysable tannins are widespread in some plant foods, e.g., grapes and wines, and contribute important organoleptic qualities, they have 


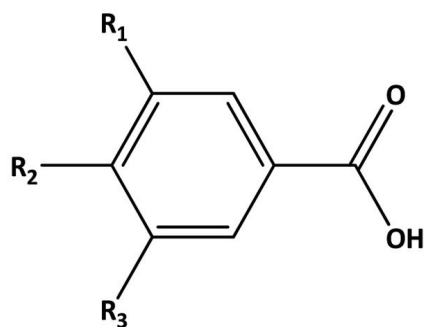

Acid

$\mathrm{R}_{1}$

$\mathrm{R}_{2}$

$\mathrm{R}_{3}$

\begin{tabular}{llll}
\hline -Hydroxybenzoic & $\mathrm{H}$ & $\mathrm{OH}$ & $\mathrm{H}$ \\
3, 4-dihydroxybenzoic & $\mathrm{H}$ & $\mathrm{OH}$ & $\mathrm{OH}$ \\
Vanillic & $\mathrm{OCH} 3$ & $\mathrm{OH}$ & $\mathrm{H}$ \\
Syringic & $\mathrm{OCH} 3$ & $\mathrm{OH}$ & $\mathrm{OCH} 3$ \\
Gallic & $\mathrm{OH}$ & $\mathrm{OH}$ & $\mathrm{OH}$ \\
Protocatechuic acid & $\mathrm{H}$ & $\mathrm{OH}$ & $\mathrm{OH}$
\end{tabular}

Figure 4. Structures of common benzoic acid derivatives.

received little attention in terms of their impact on human health (Beecher, 2003). Catechin and epicatechin are the two important proanthocyanidin monomers in human foods of plant origin $(\mathrm{Gu}$ et al., 2003). These can combine to create esters such as catechin/ epicatechin gallate. Fifteen subclasses of proanthocyanidins have been identified (Porter, 1993). However, only three appear to be prominent in human foods of plant origin, procyanidins ([epi]catechin polymers), prodelephinidins ([epi]gallocatechin polymers) and propelargonidins ([epi]afselechin) polymers or their mixtures (Gu et al., 2003). Tannins can bond with sugars and dietary proteins as well as with enzymes to create glycosides and polyphenolic proteins (Naczk et al., 1996; Naurato, et al., 1999). Due to this complex, the digestibility of protein is reduced either by direct precipitation or by inhibition of enzyme activity (Ferguson, 2001).

\subsection{Lignans}

The lignans comprise a class of natural plant products, which are<smiles>O=c1oc2c(O)c(O)cc3c(=O)oc4c(O)c(O)cc1c4c23</smiles>

Figure 5. Structure of ellagic acid (dimeric derivative of gallic acid).<smiles>[R]c1cc(/C=C/C(=O)O)cc([R3])c1[R]</smiles>

Aci

$\begin{array}{lll}\mathrm{R}_{1} & \mathrm{R}_{2} & \mathrm{R}_{3}\end{array}$

p-Coumaric

$\mathrm{H}$

$\mathrm{OH}$

$\mathrm{H}$

Caffeic

$\mathrm{H}$

$\mathrm{OH}$

$\mathrm{OH}$

Ferulic

$\mathrm{OCH}_{3}$

$\mathrm{OH}$

$\mathrm{H}$

Sinapic

$\mathrm{OCH}_{3}$

$\mathrm{OH}$

$\mathrm{OCH}_{3}$

Figure 6. Structures of common cinnamic acid derivatives.

derived from cinnamic acid derivatives by coupling of two $\mathrm{C}_{6}-\mathrm{C}_{3}$ molecules (Moss, 2000) (Figure 9). Two most extensively studied dietary lignans are secoisolariciresinol and matairesinol (Figure 10). These plant lignans are converted to mammalian lignans by fermentation in the large intestine where matairesinol is converted to enterolactone and secoisolariciresinol to enterodiol, and the latter is, to some degree, oxidized to enterolactone (Setchell and Adlercreutz 1988). Plant lignans have been analysed in fruits, vegetables, cereal products, tea and coffee (Liggins et al., 2000; Mazur, 1998, Mazur et al., 1998; Nesbitt and Thompson, 1997, Nurmi, et al., 2003; Rodríguez-García et al., 2019). The most abundant lignans in sesame seeds (Sesamum indicum Linn, Pedaliaceae), sesamin and sesamolin, are reported to lack any appreciable in vitro antioxidative activity. Rather, the high antioxidative properties of sesame seed appear to be related to lignans, such as sesamol (Budowski 1950), sesamolinol, pinoresinol, (Fukuda et al., 1985, 1986) and sesaminol (Kang et al., 1998a; Kang, et al., 1998b). These compounds also have inhibitory effects on membrane lipid peroxidation, the microsome peroxidation induced by ADP-Fe ${ }^{3+} / \mathrm{NADPH}$ (Kang et al., 1998b) and the oxidation of LDL induced by copper ions (Kang et al., 1999) or 2,2'-azobis (2,4-dimethylvaleronitrile) (AMVN) (Kang et al., 1998b) and show syn-<smiles>O=C(/C=C/c1ccc(O)c(O)c1)OC1CC(O)(C(=O)O)CC(O)C1O</smiles>

Figure 7. Structure of chlorogenic acid. 


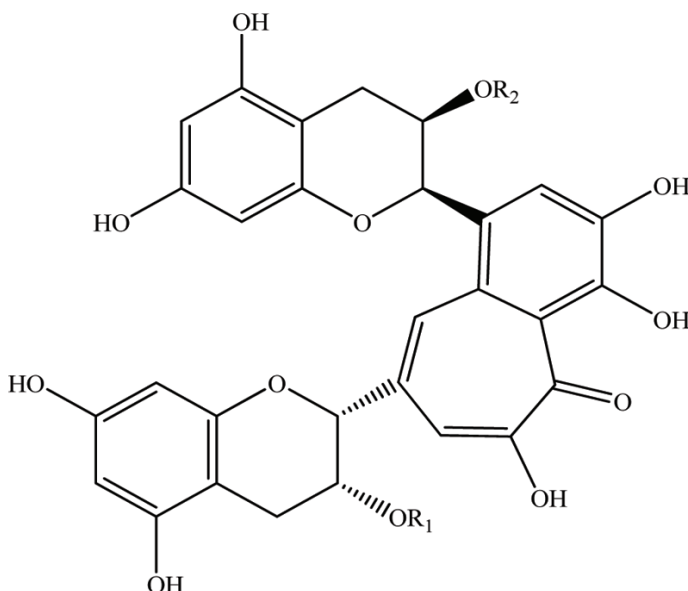

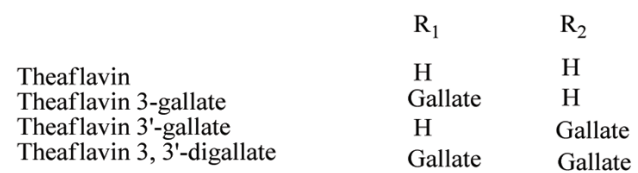<smiles>O=C1CC([C@H]2Cc3cc(O)cc(O)c3C[C@H]2O)CC(O)C1=O</smiles>

Thearubigin

Figure 8. General structures for theaflavins and their specific compounds and thearubigin. Source: Adapted from Beecher, (2003).

ergistic effects in elevating the levels of vitamin $\mathrm{E}$ in rat liver and plasma (Kamal-Eldin et al., 1995; Yamashita et al., 1995). Studies have indicated that products made from wholemeal rye flour, which contains plant lignans, may have protective effects on the development of some hormone-dependent cancers (Adlercreutz et al., 1995).

\subsection{Stilbenoids}

Stilbenes possess a C6-C2-C6 basic carbon skeleton containing 1,2-diphenylethylene as a functional group. Resveratrol (3,5,4'-trihydroxystilbene) (Figure 11) is a member of the stilbene family produced in some fruits occurring in both free and glycoside forms (Nichenametla et al., 2006). These hydroxystilbenes are of particular interest in grapes and red wine (Dercks and Creasy, 1989; Celotti et al., 1996) in which it is considered to impart health benefits such as antioxidant, anti-inflammatory, cardiovascular health and cancer chemopreventive activities (Jang et al., 1997; Donnelly et al., 2004; Oh and Shahidi, 2017; Oh and Shahidi 2018).

\subsection{Hydroxytyrosol}

Tyrosol is well reported in wine and virgin olive oil (Cornwell and Ma, 2008; Fragopoulou et al., 2007; Zhou et al., 2017; Sun et al., 2018). Olive mill wastewater is a rich source for hydroxytyrosol and tyrosol (Feki et al., 2006; Obied et al., 2005) (Figure 12).

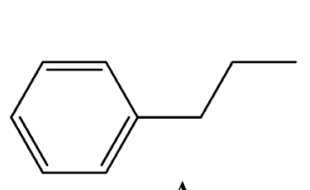

A

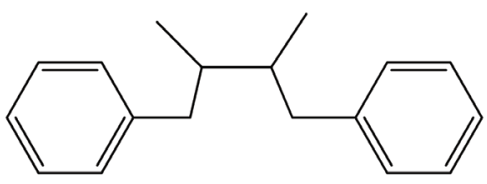

B
Figure 9. Parent structure of lignan [coupling (B) of two C6-C3 molecule (A)].
Although plant-derived polyphenolic compounds have several beneficial effects, they have some negative attributes include offflavour and taste, discolouration, carcinogenic activity and antinutritional activity (Ssonka and Xia, 2005). Phenolic compounds in Agri-food by-products are given in Table 1 .

\section{By-products of fruits}

Fruits belong to food products appreciated by dieteticians and nutritionists. Majority of fruits are rich source of vitamin C, carotenoids and polyphenolic compounds. By-products of fruits generally show a higher antioxidant activity than vegetable by-products (Wijngaard et al., 2009).

\subsection{Apple}

Apples are widely consumed fruit worldwide, and they are one of the best sources of antioxidant and phenolic compounds, including quercetin, catechin, phlorizdin and chlorogenic acid (Boyer, and Liu, 2004; Eberhardt et al., 2000). Apart from among different varieties of apples, contents of phenolic compounds vary greatly between the flesh and the peel of the apple and predominantly localized in the peels (54\%) (Escarpa and Gonzalez, 1998; Schieber et al., 2003a; Vrhosek et al., 2004; Eberhardt et al., 2000; Wolfe et al., 2003).

Apple peel is known to contain several quercetin and phloretin glycosides along with catechins, procyanidins, phloridzin, caffeic acid, chlorogenic acid, cyanidin glycosides (cyanidin-3-glucoside or kuromanin) (Andrade et al., 1998; Burda et al., 1990; Escarpa and Gonzalez, 1998; Golding et al., 2001; van der Sluis et al., 2001; Suarez-Valles et al., 1994; Whale and Zora, 2007; Wijngaard et al., 2009; Sekhon-Loodu et al., 2013). Lommen et al. (2000) individually identified and confirmed the presence of quercetin- $O$ glycosides namely, quercetin-3- $\alpha$-1-rhamnosyl-(1-6)- $\beta$-d-glucoside (rutin), quercetin-3- $\beta$-d-galactoside (hyperin), quercetin-3- $\beta$-d- 


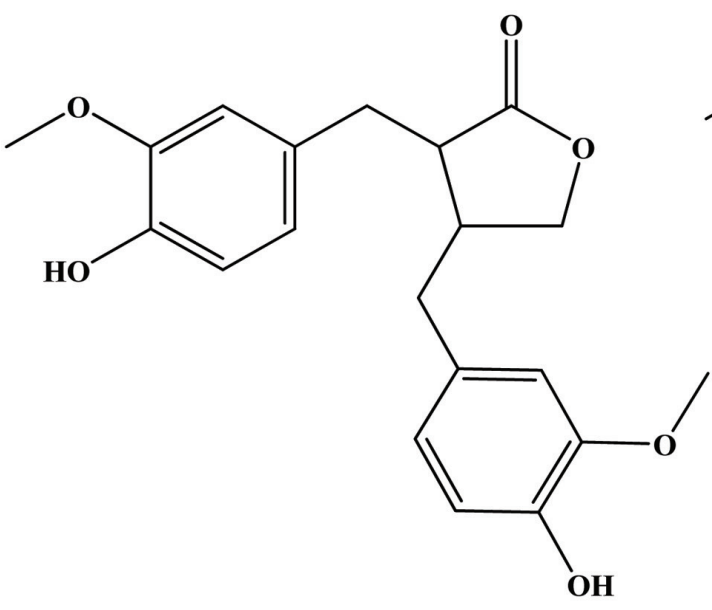

Matairesinol<smiles>COc1cc(CC(CO)C(CO)Cc2ccc(O)c(OC)c2)ccc1O</smiles>

Secoisolariciresinol

Figure 10. Structure of major dietary lignans.

glucoside (isoquercitrin), quercetin-3- $\beta$-d-xyloside (reynoutrin), quercetin-3- $\alpha$-1-arabinofuranoside (avicularin), and quercetin-3$\alpha$-l-rhamnoside (quercitrin) and phloretin $O$-glycosides namely, phloretin-2- $\beta$-d-glucoside (phloridzin) and the 2- $\beta$-d-xylosyl-(1$6)-\beta$-d-glucoside in an extract of apple peel using directly coupled HPLC-NMR-MS. Authors found that concentrations of these individual compounds were between 0.2 and $5 \mathrm{mg} / \mathrm{g}$ of apple peel. According to Wolfe and Liu (2003), total phenolic concentrations of apple peel were $33 \mathrm{mg} / \mathrm{g}$ dry weight. It has been shown that processing methods influence the antioxidant properties of fractionated apple peel polyphenols (Sekhon-loodu et al., 2013).

Apple seeds are recognized as a rich source of valuable compounds (Awad et al., 2000; Fromm et al., 2013; Jham, 1996; Lu and Foo, 1998; Schieber et al., 2003a). They are yielding more than $2 \mathrm{~g}$ of polyphenolics per $\mathrm{kg}$ in total (Schieber et al., 2003a). $\mathrm{Lu}$ and Foo (1998) identified two major bioactive compounds (Figure 13), namely amygdalin and phloridzin, the latter making up to $75 \%$ of the total polyphenols and some minor components namely, chlorogenic acid, $p$-coumarylquinic acid, 3-hydroxyphloridzin, phloretin-2'-xyloglucoside and six quercetin glycosides (arabinoside, galactoside, glucoside, rhamnoside, rutinoside and xyloside) while flavonoids and procyanidins were not detected. In another study, Schieber et al. (2003a) found phloridzin, making up $90 \%$ of total phenolics, along with appreciable amount of chlorogenic acid, phloretin xyloglucoside, epicatechin procyanidin B2 and a number of quercetin glycosides. While quercetin glycosides

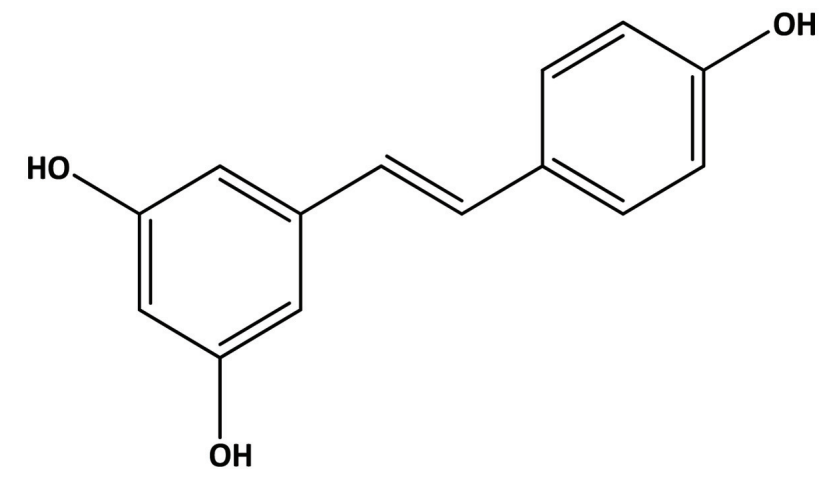

Figure 11. Structure of trans-resveratrol (trans-3,5,4-trihydroxystilbene). were not detected by Awad et al. (2000), they found low levels of cyanidin-3-galactoside.

Apple pomace, a by-product of apple juice industry, has been shown to be a good source of polyphenols, minerals and dietary fibre (Boyer and Liu, 2004; Ćetković et al., 2008; Figuerola et al., 2005; Foo and Lu, 1999; Lommen et al., 2000; Lu and Foo, 1997, 1998; Reis et al., 2012; Schieber et al., 2001a; Sudha et al., 2007; Wijngaard et al., 2009). It mainly consists of peels and cores, which are central part of an apple, contain seeds. When an apple is consumed, often the core is not eaten as it is woodier. Seeds constitute approximately $2-3 \%$ of the total weight of apple pomace (Carson et al., 1994). Lyophilised apple pomace was found to contain to about $118 \mathrm{mg} / \mathrm{g}$ of phenolics (Schieber et al., 2003a).

Predominant polyphenolic compounds in commercial apple pomace are phloridzin and chlorogenic acid, followed by quercetin glycosides (quercetin 3-galactoside, quercetin 3-rhamnoside, quercetin 3-glucoside, quercetin 3-xyloside, quercetin 3-rutinoside, and quercetin 3-arabinoside) (Ćetković et al., 2008; Schieber et al., 2003a; Schieber et al., 2001a) (Figure 14). In addition, authors were able to identify epicatechin, procyanidin B2, catechin, $p$-coumaroylquinic acid, $p$-coumaric acid, ferulic acid, phloretin xyloglucoside, quercetin, and phloretin. While chlorogenic acid was not detected by $\mathrm{Lu}$ and Foo (1997), they found that quercetin-glycoside (quercetin-3-galactoside) and dihydrochalcone (phloridzin) being the most abundant compounds and dihydrochalcone-glycoside (phloretin-2-xyloglucoside) being the least abundant.

Since some phenolic constituents have been demonstrated to exhibit strong antioxidant activity in vitro (Lu and Foo, 2000), commercial exploitation of apple pomace for the recovery of these compounds seems promising. In addition, Alongi et al. (2019) substituted $10-20 \%$ of wheat flour with apple pomace, which contained dietary fiber and phenolic compounds, when they made<smiles>OCCc1ccc(O)cc1</smiles>

Figure 12. Structure of hydroxytyrosol. 
Table 1. Phenolic compounds in agri-food by-products

\begin{tabular}{|c|c|c|c|}
\hline By-products & Major phenolic compounds & Total Phenolic content ${ }^{a}$ & Reference \\
\hline Apple peel & $\begin{array}{l}\text { Quercetin glycosides, phloretin } \\
\text { glycosides, catechins, procyanidins, } \\
\text { phloridzin, caffeic acid, chlorogenic } \\
\text { acid, cyanidin glycosides }\end{array}$ & $33.42 \mathrm{mg} / \mathrm{g}(\mathrm{GAE})$ & $\begin{array}{l}\text { Wijngaard et al. (2009), } \\
\text { Wolfe and Liu (2003) }\end{array}$ \\
\hline Apple seed & Amygdalin, phloridzin & $2.17 \mathrm{mg} / \mathrm{g}$ & $\begin{array}{l}\text { Schieber et al. (2003a), } \\
\text { Lu and Foo (1998) }\end{array}$ \\
\hline Apple pomace & $\begin{array}{l}\text { Phloridzin, chlorogenic acid, } \\
\text { quercetin glycosides }\end{array}$ & $4.22-8.67$ (CGE) & $\begin{array}{l}\text { Schieber et al. (2003a), } \\
\text { Ćetković et al. (2008) }\end{array}$ \\
\hline Berry pomace & $\begin{array}{l}\text { Cyanidin-3-O-glucoside, } \\
\text { quercetin-3-O-glucoside, gallic } \\
\text { acid, protocatechuic acid }\end{array}$ & 21.7-47.4 mg/g (GAE) & Zhou et al. (2009) \\
\hline Grape skin & $\begin{array}{l}\text { Catechin, epicatechin, epicatechin } \\
\text { gallate, epigallocatechin }\end{array}$ & & Souquet et al. (1996) \\
\hline Grape seed & $\begin{array}{l}\text { Catechin, epicatechin, epicatechin-3-O- } \\
\text { gallate, dimeric procyanidins } \mathrm{B} 2 \text { and } \mathrm{B} 3\end{array}$ & 325.37-811.95 mg/g (GAE) & Anastasiadi et al. (2009) \\
\hline Grape stem & trans-Resveratrol, e-viniferin & 367.1-494.2 mg/g (GAE) & $\begin{array}{l}\text { Anastasiadi et al. (2009), } \\
\text { Püssa et al. (2006) }\end{array}$ \\
\hline Grape pomace & $\begin{array}{l}\text { Anthocyanins, catechins, } \\
\text { flavonol glycosides, phenolic } \\
\text { acids, trans-resveratrol }\end{array}$ & 107.12-376.71 mg/g (GAE) & $\begin{array}{l}\text { Anastasiadi et al. (2009), } \\
\text { Yi et al. (2009) }\end{array}$ \\
\hline Citrus by products (peel) & $\begin{array}{l}\text { Hesperidin, narirutin, naringin } \\
\text { eriocitrin and their glycosides, } \\
\text { hydroxycinnamic acid }\end{array}$ & $24 \mathrm{mg} / \mathrm{g}(\mathrm{GAE})$ & $\begin{array}{l}\text { Manthey and Grohmann } \\
\text { (2001), Peterson et al. } \\
\text { (2006), Sultana et al. (2008) }\end{array}$ \\
\hline Mango peel & $\begin{array}{l}\text { Flavonol-O-glycosides, } \\
\text { xanthone- } C \text {-glycosides }\end{array}$ & 55-110 mg/g (GAE) & $\begin{array}{l}\text { Ajila et al. (2007), } \\
\text { Schieber et al. (2003b) }\end{array}$ \\
\hline Mango seed & Gallic acid, ellagic acid, gallates & $117 \mathrm{mg} / \mathrm{g}(\mathrm{GAE})$ & $\begin{array}{l}\text { Puravankara et al. (2000), } \\
\text { Soong and Barlow (2004) }\end{array}$ \\
\hline Pomegranate peel & $\begin{array}{l}\text { Punicalagin, punicalin, ellagic } \\
\text { acid, gallagic acid, quercetin, } \\
\text { kaemferol, myricetin }\end{array}$ & 364 mg/g (GAE) & Sultana et al. (2008) \\
\hline Banana peel & $\begin{array}{l}\text { Dopamine, flavonone } \\
\text { glycoside, naringin, rutin }\end{array}$ & $11 \mathrm{mg} / \mathrm{g}(\mathrm{GAE})$ & $\begin{array}{l}\text { Kanazawa and Sakakibara } \\
\text { (2000), Sultana et al. (2008) }\end{array}$ \\
\hline Banana bracts & Anthocyanin & & Pazmino-Duran et al. (2001) \\
\hline Longan seeds & Gallic acid, ellagic acid & & $\begin{array}{l}\text { Soong and Barlow } \\
(2005,2006)\end{array}$ \\
\hline Almond hull & $\begin{array}{l}\text { Catechin, procatechuic acid, } \\
\text { chlorogenic acid, cryptochlorogenic } \\
\text { acid, prenylated benzoic acid }\end{array}$ & 35.9-166.7 mg/g (GAE) & $\begin{array}{l}\text { Sang et al. (2002a), } \\
\text { Sfahlan et al. (2009) }\end{array}$ \\
\hline Almond green shell cover & $\begin{array}{l}\text { Caffeic acid, } p \text {-coumaric acid, } \\
\text { ferulic acid, sinapic acid }\end{array}$ & $\begin{array}{l}18.5-62.7 \mathrm{mg} / \mathrm{g}(\mathrm{GAE}) \\
71 \mathrm{mg} / \mathrm{g}(\mathrm{CE})\end{array}$ & $\begin{array}{l}\text { Sfahlan et al. (2009), } \\
\text { Wijeratne et al. (2006), } \\
\text { Siriwardhana and } \\
\text { Shahidi (2002) }\end{array}$ \\
\hline Almond skin & $\begin{array}{l}\text { Flavan-3-ols, flavonol } \\
\text { glycosides, dihydroflavonols, } \\
\text { flavonones, phenolic acid }\end{array}$ & 88 mg/g (CE) & $\begin{array}{l}\text { Siriwardhana and } \\
\text { Shahidi (2002) }\end{array}$ \\
\hline Hazelnut skin & Phenolic acids & 577.7 mg/g (CE) & Shahidi et al. (2007) \\
\hline Hazelnut shell & & $214.1 \mathrm{mg} / \mathrm{g}(\mathrm{CE})$ & Shahidi et al. (2007) \\
\hline Hazelnut green leafy cover & & $127.3 \mathrm{mg} / \mathrm{g}(\mathrm{CE})$ & Shahidi et al. (2007) \\
\hline Hazelnut leaf & & $134.7 \mathrm{mg} / \mathrm{g}(\mathrm{CE})$ & Shahidi et al. (2007) \\
\hline Cashew nut shell liquid & Anacardic acids, cardols, cardanols & $353.6 \mathrm{mg} / \mathrm{g}$ & Trevisan et al. (2006) \\
\hline
\end{tabular}


Table 1. Phenolic compounds in agri-food by-products - (continued)

\begin{tabular}{|c|c|c|c|}
\hline By-products & Major phenolic compounds & Total Phenolic content ${ }^{\mathrm{a}}$ & Reference \\
\hline Cashew fibre & Alkyl phenols & $6.1 \mathrm{mg} / \mathrm{g}$ & Trevisan et al. (2006). \\
\hline Cashew skin & Epicatechin, catechin & $243 \mathrm{mg} / \mathrm{g}$ & Kamath and Rajini (2007) \\
\hline Pistachio hull & Gallic acid & 32.8-34.7 mg/g (TAE) & $\begin{array}{l}\text { Goli et al. (2005), } \\
\text { Vahabzadeh et al. (2004) }\end{array}$ \\
\hline Walnut skin (pellicle) & $\begin{array}{l}\text { Juglone, syringic acid, ellagic acid, } \\
\text { hydrolysable tannins, condensed tannins }\end{array}$ & 230-490 mg/g (GAE) & $\begin{array}{l}\text { Colaric et al. (2005), } \\
\text { Labuckas et al. (2008) }\end{array}$ \\
\hline Red onion dry peel & Quercetin, quercetin glycoside & 384.7 mg/g (GAE) & Singh et al. (2009) \\
\hline Tomato skin & $\begin{array}{l}\text { Quercetin, kaempferol, rutin, } \\
\text { phenolic acids, naringenin }\end{array}$ & $0.29 \mathrm{mg} / \mathrm{g}(\mathrm{GAE})$ & Toor and Savage (2005) \\
\hline Potato peel & $\begin{array}{l}\text { Chlorogenic acid, gallic acid, } \\
\text { protocatechuic acid, caffeic acid }\end{array}$ & $2.9-4.2 \mathrm{mg} / \mathrm{g}$ & Singh and Rajini (2008) \\
\hline Red Beet pomace & $\begin{array}{l}\text { l-Tryptophan, } p \text {-coumaric } \\
\text { acid, ferulic acid }\end{array}$ & 87-151 mg/g (GAE) & Peschel et al. (2006) \\
\hline Carrot peel & Chlorogenic acid, caffeic acid & $\begin{array}{l}13.8 \mathrm{mg} / \mathrm{g}(\mathrm{GAE}) \\
9.79 \mathrm{mg} / \mathrm{g}(\mathrm{GAE})\end{array}$ & $\begin{array}{l}\text { Chantaro et al. (2008), Zhang } \\
\text { and Hamauzu (2004) }\end{array}$ \\
\hline Pumpkin peels & $\begin{array}{l}\text { Vanillic acid, } p \text {-coumaric } \\
\text { acid, sinapic acid }\end{array}$ & & $\begin{array}{l}\text { Schmidtlein and } \\
\text { Herrmann (1975) }\end{array}$ \\
\hline Pumpkin seed hull & $\begin{array}{l}p \text {-Hydroxybenzoic acid, protocatechuic } \\
\text { acid, vanillic acid, trans-p-coumaric } \\
\text { acid, ferulic acid, trans-sinapic acids, } \\
\text { syringic acid, } p \text {-hydroxybenzaldehyde }\end{array}$ & & Peričin et al. (2009) \\
\hline Pumpkin oil cake & $\begin{array}{l}p \text {-Hydroxybenzoic acid, protocatechuic } \\
\text { acid, vanillic acid, trans- } p \text {-Coumaric } \\
\text { acid, ferulic acid, trans-sinapic acids, } \\
\text { caffeic acid, } p \text {-hydroxybenzaldehyde }\end{array}$ & & Peričin et al. (2009) \\
\hline Artichoke by-products & $\begin{array}{l}\text { Caffeoylquinic acid derivatives, luteolin } \\
\text { and apigenin glycosides, cynaroside }\end{array}$ & & $\begin{array}{l}\text { Liorach et al. (2002), } \\
\text { Zhu et al. (2004) }\end{array}$ \\
\hline Peanut skin & $\begin{array}{l}\text { Caffeic acid, chlorogenic acid, ferulic } \\
\text { acid and coumaric acid, catechins, } \\
\text { procyanidins, resveratrol }\end{array}$ & $\begin{array}{l}90-125 \mathrm{mg} / \mathrm{g}, \\
144.1-158.6 \mathrm{mg} / \mathrm{g}\end{array}$ & $\begin{array}{l}\text { Nepote et al. (2002), } \\
\text { Yu et al. (2005) }\end{array}$ \\
\hline Peanut hull & Luteolin & $7.3-41.8 \mathrm{mg} / \mathrm{g}$ & Duh et al. (1992) \\
\hline Bean seed coat & $\begin{array}{l}\text { Condensed tannins, anthocyanins, } \\
\text { quercetin, kaempferol glycosides }\end{array}$ & $6.7-270 \mathrm{mg} / \mathrm{g}(\mathrm{CE})$ & $\begin{array}{l}\text { Madhujith and } \\
\text { Shahidi (2005a) }\end{array}$ \\
\hline Corn bran & Ferulic acid $p$-coumeric acid and vannilin & $50 \mathrm{mg} / \mathrm{g}$ (GAE) & Ou and Kwok (2004) \\
\hline Corn tassel & & $0.74-1.57$ mg/g (GAE) & Mohsen and Ammar (2009) \\
\hline Corn cob & & $12-42 \mathrm{mg} / \mathrm{g}(\mathrm{GAE})$ & Sultana et al. (2007) \\
\hline Wheat bran & $\begin{array}{l}\text { Ferulic, syringic, } p \text {-hydroxybenzoic, } \\
\text { vanillic, coumaric, caffeic, } \\
\text { salicylic, trans-cinnamic acids }\end{array}$ & $4 \mathrm{mg} / \mathrm{g}(\mathrm{GAE})$ & $\begin{array}{l}\text { Kim et al. (2006), Ou } \\
\text { and Kwok (2004), } \\
\text { Sultana et al. (2008) }\end{array}$ \\
\hline Rice bran & & 4 mg/g (GAE) & Sultana et al. (2008) \\
\hline Rice hull & $\begin{array}{l}\text { Phytic acid, vannillic acid, syringic } \\
\text { acid, ferulic acid, iosvitexin }\end{array}$ & $2 \mathrm{mg} / \mathrm{g}(\mathrm{GAE})$ & Sultana et al. (2008) \\
\hline Oat hull & $\begin{array}{l}\text { Ferulic, } p \text {-coumaric, } p \text {-hydroxybenzoic, } \\
\text { vanillic, o-coumaric, sinapic, } \\
\text { 4-hydroxyphenylacetic, salicylic } \\
\text { acids, vanillin, catechol }\end{array}$ & $0.56 \mathrm{mg} / \mathrm{g}$ & Xing and White (1997) \\
\hline Buckwheat hull & $\begin{array}{l}\text { Quercetin, rutin, hyperin, vitexin, } \\
\text { isovitexin, protocatechuic acid, } \\
\text { 3,4-dihydroxybenzaldehyde, } \\
\text { proanthocyanidins }\end{array}$ & $39 \mathrm{mg} / \mathrm{g}$ & Watanabe et al. (1997) \\
\hline
\end{tabular}


Table 1. Phenolic compounds in agri-food by-products - (continued)

\begin{tabular}{|c|c|c|c|}
\hline By-products & Major phenolic compounds & Total Phenolic content $\mathrm{t}^{\mathrm{a}}$ & Reference \\
\hline Canola Hull & Phenolic acids, condensed tannin & 94.3-296 mg/g (SE) & $\begin{array}{l}\text { Amarowicz et al. (2000a), } \\
\text { Naczk et al. (2005) }\end{array}$ \\
\hline Olive leaves & $\begin{array}{l}\text { Oleuropein, hydroxytyrosol, } \\
\text { luteolin-7-glucoside, apigenin- } \\
\text { 7-glucoside, verbascoside }\end{array}$ & & Benavente-Garcia et al. (2000) \\
\hline Olive mill waste water & $\begin{array}{l}\text { Hydroxytyrosol, tyrosol, oleuropein, } \\
\text { dimethyloleuropein, verbascoside, } \\
\text { catechol, hydroxycinnamic acids, } \\
\text { 4-hydroxybenzoic acid }\end{array}$ & & $\begin{array}{l}\text { Bianco et al. (2003), Casa } \\
\text { et al. (2003), Feki et al. } \\
\text { (2006), Lesage-Meesen } \\
\text { et al. (2001), Ramos- } \\
\text { Cormenzana et al. (1996) }\end{array}$ \\
\hline Sunflower seed shell & $\begin{array}{l}\text { Chlorogenicacid, o-cinnamic } \\
\text { acid, protocatechuic acid, caffeic } \\
\text { acid, ferulic acid, syringic acid }\end{array}$ & $0.4-0.86 \mathrm{mg} / \mathrm{g}$ & $\begin{array}{l}\text { Leonardis et al. (2005), } \\
\text { Weisz et al. (2009), }\end{array}$ \\
\hline Sesame seed coat & Sesamin, sesamolin & 146.6-29.7 mg/g (CE) & $\begin{array}{l}\text { Chang et al. (2002), } \\
\text { Shahidi et al. (2006) }\end{array}$ \\
\hline Soybean cake & $\begin{array}{l}\text { Daidzein, genistein and } \\
\text { glycitein and their glucosides, } \\
\text { acetylglucoside, malonylglucoside }\end{array}$ & & Kao et al. (2008) \\
\hline Flax shive & $\begin{array}{l}p \text {-Coumaric acid, ferulic acid, } p \text { - } \\
\text { hydroxybenzaldehyde, vanillic acid, } \\
\text { syringic acid, vanillin, acetovanillone }\end{array}$ & & Buranov and Mazza (2009) \\
\hline Cocoa leaves & $\begin{array}{l}\text { Epicatechin, epigallocatechin } \\
\text { gallate, epigallocatechin, gallic } \\
\text { acid, epicatechin gallate }\end{array}$ & $284 \mathrm{mg} / \mathrm{g}$ & Osman et al. (2004) \\
\hline
\end{tabular}

Abbbreviations: GAE: gallic acid equivalents; CE: catechin equivalents; TAE: tannic acid equivalents; SE: sinapic acid equivalents; CGE: chlorogenic acid equivalents. aExpressed as dry weight.

short dough biscuits and evaluated their effect on glycemic index. They found that the apple pomace-added biscuit reduced glycemic index $7-14 \%$ and suggested that apple pomace could be used as a functional bakery ingredient.

\subsection{Berry}

Berry pomace (pressed skins, pulp residue, seeds and stems) is the press residue remaining when fruits are processed for juice, wine or other products. The phenolic composition of by-products mainly depends on processing techniques such as duration of skin contact, crushing, pressing and others (Su and Silva, 2006). Berries such as highbush, lowbush and rabbiteye blue berry have polyphenolic components mainly in the skin. Wide variety of polyphenolic compounds, such as anthocyanin, flavonol and polyphenolic acids (gallic and protocatechuic acid) have been reported in bay berry pomace and the total phenolic content ranged from 21.7 to $47.4 \mathrm{mg}$ (gallic acid equivalents) per gram dry matter (Zhou et al., 2009).

According to Ayoub et al. (2016), gallic acid and its derivatives were one of the major phenolic acid groups in the seed meals of blackberry, black raspberry, and blueberry. Quercetin and its derivatives were the most dominant flavonoids in blackberry and black raspberry seed meals. On the other hand, procyanidin dimer $\mathrm{B}$ was the most abundant flavonoids in the seed meal of blue berry. Among seed meals, blackberry seed meal showed larger amount of phenolics than the other seed meals.

Anthocyanins are especially abundant in some berries, including bilberries and black currants among others (Kähkönen et al., 2003). According to the study of Zhou et al. (2009), the most abun- dant anthocyanin was cyanidin-3-O-glucoside $(3,073-6,219.2 \mathrm{mg} /$ $\mathrm{kg}$ dry matter) and the main flavonol was quercetin-3-O-glucoside (296-908 mg/kg dry matter) in bay berry pomace. In addition, they identified quercetin, myricetin, myricetin deoxyhexoside, quercetin deoxyhexoside, $p$-hydroxybenzoic acid, vannilic caffeic, $p$-coumaric, and ferulic acids. Their study showed that total phenolic acids (180-324 mg/kg dry weight) were much lower than the anthocyanins and total flavonols.

The total phenolic content or total anthocyanins of bay berry pomace exhibits significant positive relationship with antioxidant activity (Zhou et al., 2009). A study of Su and Silva (2006) on rabbiteye blueberry showed that pomace from wine and juice retained important phenolic concentrations and antioxidant activities compare to vinegar pomace. They found that total phenolic content of pomace of these different products ranged from 21 to $29 \mathrm{mg} / 100 \mathrm{~g}$ (gallic acid equivalents).

\subsection{Citrus fruits}

Citrus fruits are acidic fruits, which act as a fabulous source of vitamin $\mathrm{C}$ and a wide range of essential nutrients required by the body. Citrus fruit list is a real long one encompassing innumerable types of citrus fruits like lemon, orange, mandarine, grapefruit, lime, tangelo, pummelo, kumquat, clementine, leech, tangerine, minneola, tangelo, citrons, lavendergem, oroblanco, shaddock, and uglifruit among others. Citrus industry (juice and essential oil) produces large quantities of by-products such as peels and seed residues and peels represent between 50 and $65 \%$ of the total weight of the fruits (Bocco et al., 1998). In general, by-products of the citrus 


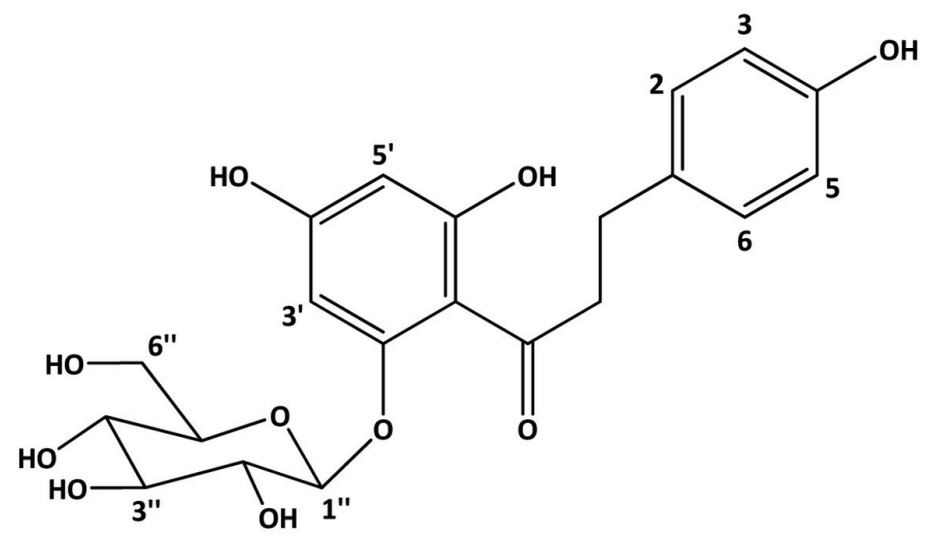

Phloridzin

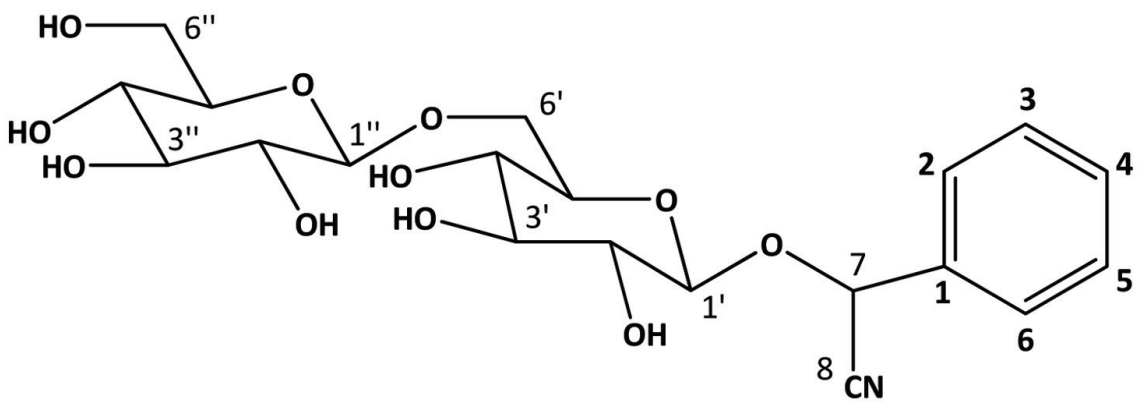

Amygdalin

Figure 13. Major bioactive compounds in Apple seed. Source: Adapted from Lu and Foo, (1998).

fruits contain different polyphenol composition in comparison to apple (Wijngaard et al., 2009).

The citrus peels are an abundant source of natural flavonoids, and contain higher amount of phenolics compared to the edible portions (Braddock, 1995; Manthey and Grohmann, 1996, 2001; Rouseff et al., 1987; Feng and Wang, 2018). Gorinstein et al. (2001) reported that the contents of total phenolics in peels of lemons, oranges, and grapefruit were $15 \%$ higher than those in the peeled fruits. Similarly, the levels of hydroxycinnamic acid were much higher in the peel than in the juice (Manthey and Grohmann, 2001). Therefore, citrus by-products release from processing plants represent rich source of phenolic compounds.

The main flavonoids found in citrus species are hesperidin, narirutin, naringin and eriocitrin (Peterson et al., 2006; Mouly et al., 1994) (Figure 15). Peel and other solid residues of lemon waste mainly contained hesperidin and eriocitrin, while the latter was predominant in liquid residues (Coll et al., 1998). Neoeriocitrin, naringin and neohesperidin are the main flavanones in the peels of sour orange $(C$. aurantium), lemon $(C$. limon) and bergamote $(C$. bergamia Fantastico) (Bocco et al., 1998; Mandalari et al., 2006). Diosmetin derivatives are the major flavones in navel orange, bergamot and lemon peels (Lin and Harnly, 2007; Mandalari et al., 2006; Miyake et al., 1997). Hesperidin is the most abundant flavonoid in valencia, navel, temple and ambersweet orange peels (Manthey and Grohmann, 1996) and naringin is the most abundant flavonoid in grapefruit peel (Wu et al., 2007). The yields of cinnamic acids, including caffeic, $p$-coumaric, ferulic, sinapic acid were generally higher than those of benzoic acids such as protocatechuic, $p$-hydroxybenzoic, vanillic acid and among all phenolic acids, the yield of ferulic acid was highest in citrus peel extract (Ma et al., 2009; Xu et al., 2008).

The total phenolic contents of citrus peels have been affected by the method of peel preparation and extraction, the operating temperature and the type of citrus peel used in the extraction ( $\mathrm{Li}$ et al., 2006a, 2006b; Senevirathne et al., 2009; Ma et al., 2009; Xu et al., 2007; Jeong et al., 2004; Oufedjikh et al., 2000). Studies revealed that heat treatment elevates the antioxidant activity of citrus peels, but the high temperatures of heat treatment destroy the flavanone glycosides (Ho and Lin, 2008; Jeong et al., 2004; Xu et al., 2007). For this reason, Xu et al. (2007) suggested that a heating temperature of less than $100{ }^{\circ} \mathrm{C}$ enhances the antioxidant activity of citrus peels, without a loss of anti-inflammatory flavanoid glucosides, which is optimal for the preparation of chen pi (the dried fruit peels of Citrus reticulate). Ho and Lin (2008) analysed the heat treating conditions for enhancing the anti-inflammatory activity of citrus peel and reported that optimal heat treatment $\left(100{ }^{\circ} \mathrm{C}\right.$ for $2 \mathrm{~h}$ ) boosted the release of some anti-inflammatory compounds, especially, polymethoxy flavones, from an unextractable form to an extractable form and thus elevated the antioxidant and antiinflammatory activities of methanol extracts of citrus fruit peels. Senevirathne et al. (2009) suggested that the high speed drying method $\left(120{ }^{\circ} \mathrm{C}, 90 \mathrm{~min}, 0.6 \mathrm{MPa}\right)$ is an effective and efficient method to transform citrus by-products into dried form and could be used in food and pharmaceutical industry as a natural antioxidant agent. According to their study, high speed drying method extract showed high amount of polymethoxylated flavones (heptamethoxyflavone and nobiletin) and flavanone (hesperidin and naritutin). Rodsamrana and Sothornvit (2019) compared ultrasonic and microwave-assisted extraction of phenolic compounds from lime peel waste, using the optiamum condition of each extraction by response surface methodology. They found that the phenolics from ultrasonic-assisted extraction showed a better antioxidant activity than that of microwave-assisted extraction. Nowadays, more attention is focused on finding an industrially applicable, efficient extraction method by keeping the stability of phenolic compounds during the treatment. 
<smiles>CCCCCCOc1c(-c2ccc(O)c(O)c2)oc2cc(O)cc(O)c2c1=O</smiles><smiles>O=C(CCc1ccc(O)cc1)c1c(O)cc(O)cc1O</smiles>

\section{Quercetin-3-glycosides}

Quercetin 3-galactoside

Quercetin 3-rhamnoside

Quercetin 3-glucoside

Quercetin 3-xyloside

Quercetin 3-rutinoside

Quercetin 3-arabinoside

\section{Phloretin}

Figure 14. Structure of quercetin-3-glycoside and phloretin identified in apple pomace. Source: Adapted from Lommen et al., (2000).

Citrus seeds and peels were found to possess high antioxidant activity (Bocco et al., 1998). Both in vitro and in vivo studies have demonstrated health protecting effects of certain citrus flavonoids (Kuo, 1996; Manthey et al., 2001; Tanaka et al., 1997). Among these flavonoids, the anti-inflammatory activity of citrus peel extract highly correlated with the content of nobiletin and tangeretin (Ho and Lin, 2008).

\subsection{Grapes}

Apart from oranges, grapes (Vitis sp., Vitaceae) are the world's largest fruit crop with a global production of around 69 million tonnes in 2006 (FAOSTAT, 2007). The seeds constitute a considerable proportion of the pomace, amounting to $38-52 \%$ on a dry matter basis (Maier et al., 2009). Apart from grape pomace (skin and seed), stem and leaves are also derived as the by-product of grape-based industries.

Grape pomace and stems contain considerable amount of diverse polyphenols such as flavonoids, phenolic acids and stilbenes (Anastasiadi et al., 2009). In grape berries, phenolic compounds are present mainly in skins and seeds. Flavonols are the most abundant phenolic compounds in grape skins, while grape seeds are rich in flavan-3-ols (Anastasiadi et al., 2009; Cheynier and Rigaud, 1986; Fuleki and Silva, 1997; Maier et al., 2009; Rodríguez Montealegre et al., 2006; Souquet et al., 2000). In particular, stems, a scarcely studied class of grape by-product are characterized as rich in the bioactive stilbenes trans-resveratrol and $\varepsilon$-viniferin (a transresveratrol dimer) (Anastasiadi et al., 2009; Püssa et al., 2006). Resveratrol and oxidation products of resveratrol have been reported in grape leaves (Borie et al., 2004; Langcake et al., 1979; Langcake and Pryce, 1977a, 1977b; Pezet et al., 2003).

Catechin, epicatechin, epicatechin gallate and epigallocatechin are the major constitutive units of grape skin tannins (Souquet et al., 1996). Although grape skins and seeds contain wide variety of condensed tannins (monomeric, oligomeric, and polymeric proanthocyanidins), grape seeds appear to contain only procyani- dins (Prieur et al., 1994) whereas the skin contains both procyanidins and prodelphinidins (Souquet et al., 1996). The polyphenolic compounds in grape seeds are essentially all flavonoids and the content of polyphenols may range from 5 to $8 \%$ by weight (Shi et al., 2003). The presence of flavan-3-ol monomers, dimers and trimers has been extensively reported in grape seeds, but they are especially rich in monomeric flavan-3-ols (+)-catechin, (-)-epicatechin and epicatechin-3-O-gallate, and dimeric procyanidins $\mathrm{B}_{2}$ and $\mathrm{B}_{3}$ (Anastasiadi et al., 2009; Santos-Buelga et al., 1995) (Figure 16). However, the distribution of flavan-3-ols is not uniform among different grape varieties (Santos-Buelga et al., 1995). It has been shown that grape seeds contain greater concentration of phenolic compounds than in the skin (Rockenbach et al. 2011). Since, procyanidins act as strong antioxidants and exert healthpromoting effects (Fuleki and Silva, 1997; Jayaprakasha et al., 2001; Kallithraka et al., 1995; Saito et al., 1998), supplementary quantities of grape seeds are added to grape extract to increase the catechin and procyanidins contents of wines (Kovac et al., 1995).

Since grape and red wine phenolics have been demonstrated to inhibit the oxidation of human low density lipoproteins (Frankel et al., 1995; Teissedre et al., 1996), a large number of investigations on the recovery of phenolic compounds from grape pomace have been initiated. From a nutritional point of view, these phenolics are highly valuable since they are absorbed to a large extent (MartinCarron et al., 1997).

Anthocyanins, catechins, flavonol glycosides, phenolic acids and alcohols and stilbenes are the principal phenolic constituents of grape pomace (Anastasiadi et al., 2009; Mazza, 1995; Mazza and Miniati, 1993; Souquet et al., 1996). The total phenol contents of grape/wine pomace vary in the range of 250 to $590 \mathrm{mg}$ (gallic acid equivalents) per $100 \mathrm{~g}$ fresh weight (Alonso et al., 2002; Murthy et al., 2002; Lafka et al., 2007; Yi et al., 2009) and anthocyanins (131-302 mg/g fresh weight) accounted for about half of the total phenolics in red grapes wine pomace (Yi et al., 2009). This relatively high content of phenolic compounds suggested that the reuse of this wine by-product is of great importance. In chardonnay grape pomace, 17 polyphenolic constituents 
<smiles>COc1ccc(C2CC(=O)c3c(O)cc(O)cc3O2)cc1O</smiles>

Hesperetin<smiles>CCC(Oc1cc(O)cc(O)c1C(=O)CCN(C)CC#N)c1ccc(O)cc1</smiles><smiles>O=C1CC(c2ccc(O)c(O)c2)Oc2cc(O)cc(O)c21</smiles>

Eriodictyol<smiles>COc1cc(O)c2c(c1)OC(c1ccc(O)cc1)CC2=O</smiles>

Narirutin (Rutinose glycoside) Naringin (Neohesperidose glycoside)<smiles>O=C1CC(c2ccc(O)c(O)c2)Oc2cc(OC3CCCCC3)cc(O)c21</smiles>

Eriocitrin (Rutinose glycoside) Neoeriocitrin (Neohesperidose glycoside)

Figure 15. Structures of flavanone aglycones and glycosides identified in citrus peel. Source: Adapted from Harborne et al., (1999).

have been reported by Lu and Foo (1999). In their earlier studies, two unusual dimeric flavanols (biflavonols) have been identified in chardonnay pomace (Foo et al., 1998). Kammerer et al. (2004) characterized the phenolic compounds of different press residues originating from red and white wine making by HPLC-MS. In this study, up to 13 anthocyanins, 11 hydroxybenzoic and hydroxycinnamic acids, and 13 catechins and flavonols as well as 2 stilbenes were identified and quantified in the skins and seeds by HPLCDAD. They observed large variability in all individual phenolic compounds related on cultivar and vintage. Further, grape skins proved to be rich sources of anthocyanins, hydroxycinnamic acids, flavanols, and flavonol glycosides, whereas flavanols were mainly present in the seeds. However, besides the lack of anthocyanins in white grape pomace, no principal differences between red and white grape varieties have been reported. A similar observation has been made by Rodríguez Montealegre et al. (2006), who reported that skins of white grape varieties presented a very similar qualitative and quantitative composition to that of red grape varieties in terms of non-anthocyanic polyphenols and the only qualitative difference was observed in the skins of white grape varieties that lacked the myricetin glycosides present in the skins of red grape varieties. Anastasiadi et al. (2009) quantified the main polyphenols of grape berries, seed, stem and vinification by-products of red and white varieties of Greek island grapes and found that seed extracts contained high concentrations of flavan-3-ols and their derivatives, whereas pomace and stem extracts consisted of significant amounts of flavonoids, stilbenes, and phenolic acids. de Camargo et al. (2014a) studied phenolic compounds present in grape juice and winemaking by-products and compared their free, esterified, and insoluble-bound forms. They reported that the insoluble-bound phenolics comprised the highest amount in both juice and winemaking by-products. Meanwhile winemaking product had a higher content of esterified phenolics than the free form, the opposite trend was found for grape juice by-products. Later, de Camargo et al. (2016) used enzymes to improve the extraction of insoluble-bound phenolics from winemaking by-products and reported that both Pronase and Viscozyme could increase soluble phenolic contents and decrease the amount of insoluble-bound phenolics. Meanwhile, Oliveira et al. (2017) found that phenolics of winemaking by-products could reduce both VLDL-cholesterol and triacylglycerol levels better than that of red wine in Winstar rats. 
<smiles>O=C(O[C@H]1Cc2c(O)cc(O)cc2O[C@@H]1c1ccc(O)c(O)c1)c1cc(O)c(O)c(O)c1</smiles>

(-)-Epicatechin-3-O-gallate<smiles>Oc1cc(O)c2c(c1)O[C@H](c1ccc(O)c(O)c1)[C@H](O)[C@H]2c1c(O)cc(O)c2c1O[C@H](c1ccc(O)c(O)c1)[C@H](O)C2</smiles>

B-type Dimer (B1)

Figure 16. Structures of flavan-3-ol monomer and dimer in grape seed. Source: Adapted from Nawaz et al., (2006).

Extraction of crushed grape pomace with a mixture of ethyl acetate and water yielded phenolic compounds displaying antioxidant activities comparable to BHT in the Rancimat test (Bonilla et al., 1999). However, drying of pomace at high temperatures may cause a significant reduction of extractable polyphenols and may also affect antioxidant activity and free radical scavenging capacity (Larrauri et al., 1997a, 1997b, 1998). Meyer et al. (1998) have showed that enzymatic treatment of grape pomace enhanced release of phenolic compounds. However, the effects of this treatment on individual phenolics were not investigated. Super heated solvents for the extraction of phenolics from grape pomace have been investigated by Palma and Taylor (1999). Furthermore, studies (Ayed et al., 1999) showed that gamma irradiation extended the shelf life of grape pomace and improved anthocyanin yields.

Resveratrol (3,5,4-trihydroxystilbene) (Langcake and Pryce, 1976 and 1977a) is a phytoalexin synthesized by grapevine leaf tissue following fungal infection and UV light irradiation. These authors have also identified oxidation products of resveratrol as $\varepsilon^{-}, \alpha-, \beta$-, and $\gamma$-viniferin, respectively, as a dimer, trimer, tetramer, and a more highly polymerized oligomer (Langcake, and Pryce, 1977b). Pterostilbene (3,5-dimethoxy-4'-hydroxystilbene) was also isolated from Vitis vinifera leaves infected by Plasmopara viticola (downy mildew) (Langcake et al., 1979). This compound was later identified in healthy grapevine berries as a constitutive stilbene (Pezet and Pont 1988).

Püssa et al. (2006) analysed the stem of frost-hardy grapevines and identified the major constituents, trans-resveratrol and its derivatives including oligomers and glucosides and minor components, stilbenoid piceatannol as well as a number of nonstilbenoid polyphenols, mostly flavan-3-ols and phenolic acids glucosides. They noticed that total polyphenol content of the grapevine stems depended on the variety, whereby the stems of cultivar. Resveratrol dimer $\varepsilon$-viniferin is considered to be a biogenetically important precursor of the other dimers like ampelopsins B, D, and F and also tetramers like hopeaphenol and isohopeaphenol, vitisins A, B, and C, and viniferol A (Takaya et al., 2002). Despite this, the overall polyphenolic composition of grapevine lignified stems remains largely unknown.

A study of Maier et al. (2009) confirmed the press residues of grape seed oil production still to be a rich source of polyphenolics with strong antioxidant activity. In this study they measured the individual phenolic compounds of grape seeds and residue of grape oil of seven cultivars by HPLC and found that phenolic profile of the seeds and residues was dominated by flavonoids, whereas phenolic acids were detected in lower amounts. They were able to identify nine flavonoids in the seeds and press residues. Among nine flavan-3-ols catechin, epicatechin, epicatechin gallate and the dimeric procyanidins $\mathrm{B} 1$ and $\mathrm{B} 2$ were the predominant components in all cultivars and quercetin, quercetin 3-O-glucoside, quercetin 3-O-galactoside and quercetin 3-O-glucuronide were also detected in some of the cultivars and they observed large variability in composition of phenolic acids. While phenolic acid profile of the press residues was more complex than that of the intact seeds. Phenolic acid profile showed that gallic acid was the predominant phenolic compound and other compounds namely coutaric acid, caftaric acid, fertaric acid, caffeic, $p$-coumaric and ferulic acids, were not detected in all cultivars and latter three were not detected in seeds.

\subsection{Mango}

Mango (Mangifera indica L. Anacardiacea) is one of the most important tropical fruits in the world and currently ranked 5 th in total world production among the major fruit crops (FAO, 2004). As mango is a seasonal fruit, about $20 \%$ of fruits are processed for products such as puree, nectar, leather, pickles, canned slices and chutney and these products experience worldwide popularity and have also gained increased importance in the US and European markets (Loelillet, 1994; Tyug et al., 2010). Major by-products of mango processing are peels and stones, amounting to $35-60 \%$ of the total fruit weight (Larrauri et al., 1996). Mango peel is constitutes about $15-20 \%$ of total weight of mango fruit. There are about $3 \times 10^{5}$ ton of dry mango seed kernels available annually in India after consumption or industrial processing of mango fruits (Narasimha Char and Azeemoddin, 1989).

The by-product of mango peel in fruit processing are a promising source of phenolic compounds (Larrauri et al., 1996, Schieber et al., 2003b). Schieber, et al. (2003b) extracted flavonol $O$-glycosides (quercetin $O$-glycosides, kaempferol $O$-glycoside) and xanthone $C$-glycosides from mango peels among the fourteen com- 
<smiles>O=c1c2cc(O)c(O)cc2oc2cc(O)c(Cl)c(O)c12</smiles>

Xanthone C-glycosides mangiferin<smiles>O=c1c2cc(O)c(O)cc2oc2c(Cl)c(O)cc(O)c12</smiles>

Isomangiferin

Figure 17. Structures of the xanthone C-glycosides mangiferin and isomangiferin. Source: Adapted from Schieber et al., (2003b).

pounds analyzed (Figures 17 and 18). The content of total phenols was higher in the peel than the pulp at any stage of mango fruit development (Lakshminarayana et al., 1979; Ueda et al., 2000).

Ajila et al. (2007) investigated the potential of peels of both raw and ripe mango of Indian varieties such as badami (alphonso) and raspuri and found that polyphenol contents in acetone extracts of raw and ripe varied from 55 to $110 \mathrm{mg} / \mathrm{g}$ dry peel (gallic acid equivalents) and in both varieties the total polyphenol content was found to be significantly higher in raw peels compared to that of ripe peels. According to Larrauri et al. (1996) the total polyphenol content in aqueous methanol extract of ripe peel of hayden variety was $70 \mathrm{mg} / \mathrm{g}$. Polyphenol content in irwin mango variety showed higher value in ripe peel compared to raw peel (Ueda et al., 2000).

The total polyphenol content in grape pomace extracts was reported to range from 68.8 to $98.3 \mathrm{mg}$ (gallic acid equivalents) per gram dry peel (Gulcan et al., 2004), which is comparable to polyphenol content in mango peels, where as in apple pomace it was reported to be $33.42 \mathrm{mg}$ (gallic acid equivalents) per gram dry peel (Wolfe et al., 2003) much lower than mango peels.

The major phenolic constituents of mango seed kernel are gallic and ellagic acids as well as gallates (Puravankara et al., 2000). Gallotannins and condensed tannin-related polyphenols are also reported in mango kernels (Arogba, 2000). Soong and Barlow (2004) investigated the total phenolic contents of pulp and seed of mango, and other fruits. They found that the seeds showed a much higher phenolic content than the edible portions. Figure 19 shows the total phenolic content of some tropical fruit seeds.

Several studies have been made on the antioxidant activity of the mango seed kernel. Puravankara et al. (2000) reported that the phenolic compounds and phospholipids were the principles of antioxidant activity in mango seed kernel. In another study, Soong et al. (2004) indicated that mango seed kernel has potent antioxidant activity with relatively high phenolic contents. Schieber et al., (2003b) and Nunez-Selles (2005) also referred that the antioxidant effect of the mango seed kernel and bark was due to the high content of polyphenols, sesquiterpenoids, phytosterols, and microelements like selenium, copper and zinc. In addition, ethanolic extracts of mango seed kernels displayed a broad antimicrobial spectrum and were more effective against gram-positive than against gram-negative bacteria. Their active component was shown to be a polyphenolic-type structure; however, its exact nature still remains to be elucidated (Kabuki et al., 2000). Guandalini et al. (2019) investigated the effect of ultrasound on extraction of phenolic compounds from mango peel. They found that the ultrasound-assisted extraction was not effective.

\subsection{Pomegranate}

Pomegranate (Punica granatum L.) is one of the oldest edible fruits widely grown in many tropical and subtropical countries (Çam et al., 2009) and is an important source of bioactive compounds (Li et al., 2006c). The edible portion of pomegranate fruit, called arils, originates from the pith and is well protected by carpellary membrane. Pith and carpellary membrane constitute $13 \%$ of pomegranate fruit composition. Pomegranate fruits are widely consumed fresh and in processed forms as juice, jams and wine peels/husk, pith and carpellary membrane and seeds are the major by-products, derived from pomegranate industry. Figure 20 shows the schematic illustration of pomegranate fruit parts.

Pomegranate seed residue mainly consists of flavol-3-ols, phenolic acids, flavonoid glycosides, and hydrolysable tannin (He et al., 2011). When compared to apple, banana and citrus peel, pomegranate peel exhibits highest phenolic content (Sultana et al., 2008). Kulkarni et al. (2004) reported that total phenolics levels increase at early stage of growth both in peel and arils of fruit, but thereafter generally decrease during maturation and reach to 3.7 and $50 \mathrm{mg}$ per gram of dry weight in arils and peel, respectively, at harvest. Pomegranate peel is the rich source of polyphenolic compounds, including mainly hydrolyzable tannins (ellagitannins), such as oligomers and punicalagin with a lesser amounts of punicalin, ellagic acid, gallagic acid and anthocyanidins (delphinidin, cyanidin, pelargonidin) and their glycosides (Aviram et al., 2008; Fischer et al., 2011; Iqbal et al., 2008; Li et al., 2006c; Chidambara Murthy et al., 2002; Singh et al., 2002; Ben et al., 1996). Next to peel, punicalagin is mainly found in pith and carpellary membrane of pomegranate (Kulkarni et al., 2004). Figure 21 shows the structures of ellagic acid derivatives. Pomegranate leaf tannins containing abundant ellagitannins, mainly ellagic acid (Lan et al., 2009). Ben et al. (1996) showed that the amount of proanthocyanidins 


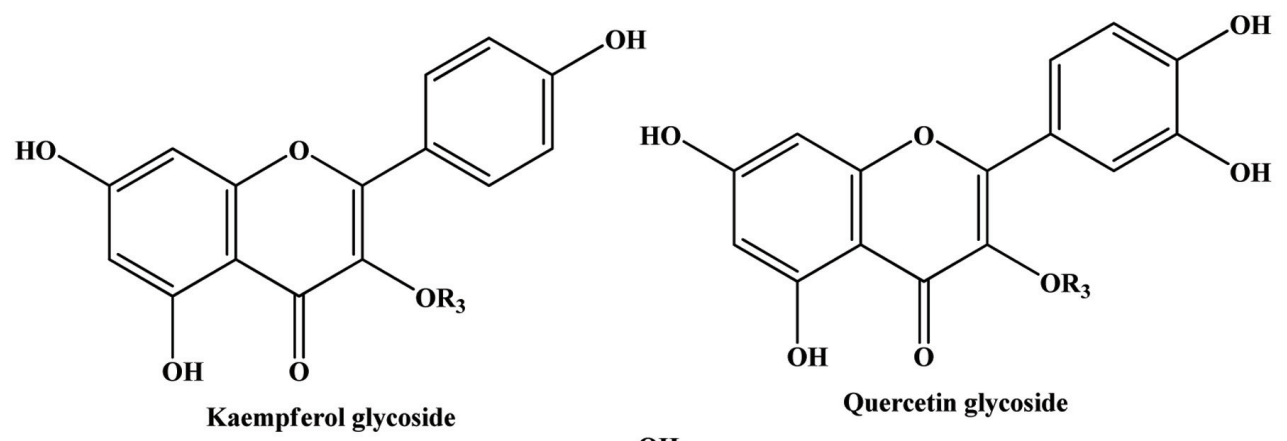<smiles>CCCCCCc1c(-c2ccc(O)c(O)c2)oc2cc(OC)cc(O)c2c1=O</smiles>

\section{$\mathbf{R}_{\mathbf{3}}$ - Glycoside moiety}

Figure 18. General structure of flavonol glycosides detected in mango peels. Source: Adapted from Schieber et al., (2003b).

represents $30 \%$ of the quantity of total polyphenols in pomegranate peel. Furthermore, they examined the influence of lighting patterns in the biosynthesis of phenolics, especially related to anthocyanidins and proanthocyanidins and found that greater exposure to sunlight increased the content of anthocyanidins in pomegranate peels and had no more effect on proanthocyanidins. In the same study, they observed the significant increase in proanthocyanidin in pomegranate peel in irrigated land compare to non-irrigated land. Sultana et al. (2008) showed that total phenolic and flavonoid content of pomegranate peel were $364 \mathrm{mg} / \mathrm{g}$ dry weight (gallic acid equivalents) and $48.9 \mathrm{mg} / \mathrm{g}$ dry weight (catechin equivalents) re- spectively. In the same study they examined the flavonol composition of pomegranate peel and found that total flavonol, myricetin, quercetin and kaempferol content were 2.6, 2.5, 0.024 and 0.102 $\mathrm{mg} / \mathrm{g}$ dry matter respectively. Pomegranate peel demonstrates higher the phenolic content and higher the antioxidant activity as compared to seed (Singh et al., 2002; Guo et al., 2003) and pulp (Li et al., 2006c; Guo et al., 2003). Apart from the antioxidant activity, pomegranate peel shows antimutagenic potency (Ghasemian et al., 2006; Negi et al., 2003). Recently, Ambigaipalan et al. (2016) examined pomegranate peels more closely. They devided the peels into three parts, namely outer skin, mesocarp, and divider mem-

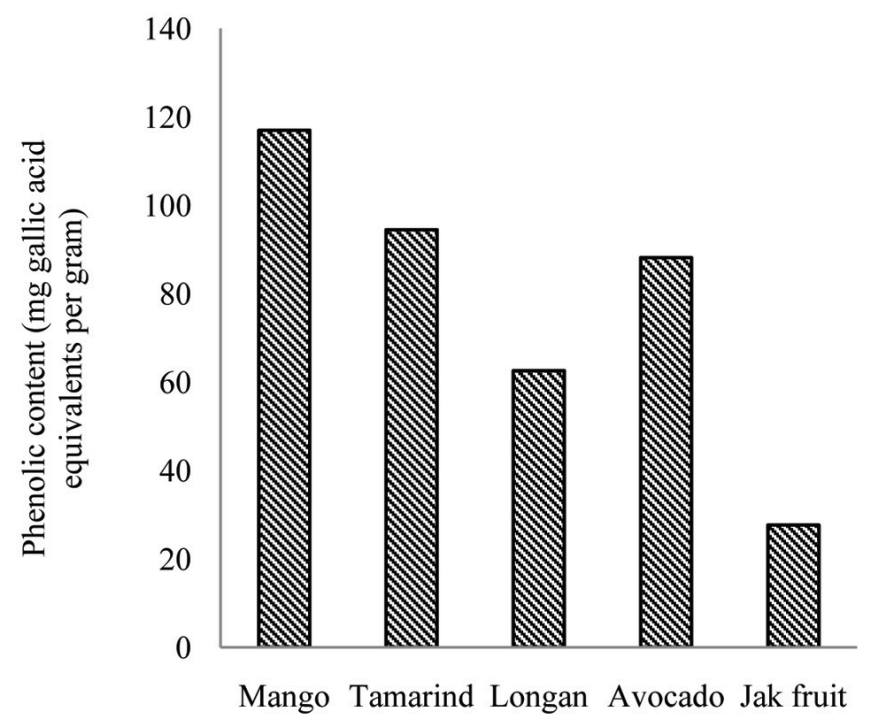

Figure 19. Total phenolic contents of seed of mango, tamarind, longan, avocado and jackfruit. Source: Adapted from Soong and Barlow, (2004). 


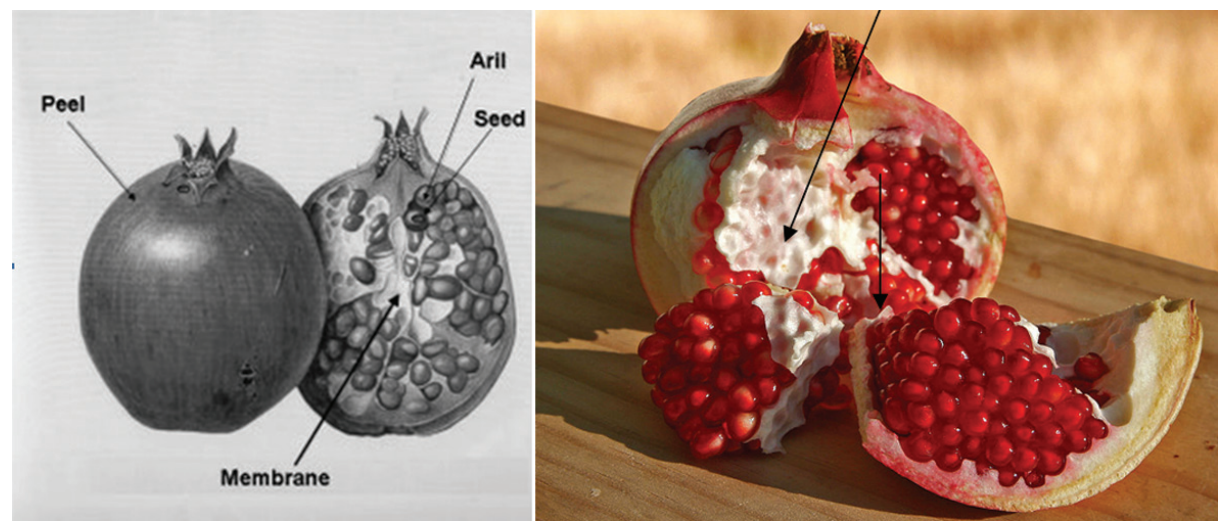

Figure 20. Schematic illustration of pomegranate fruit parts and their terminology.

brane and evaluated the phenolic contents and their antioxidant activities. Gallic acid and kaempferol 3-O-glucoside were the most abundant phenolic acid and flavonoid, respectively. The outer skin contained the highest amount of phenolic compounds followed by divider and mesocarp. In a subsequent study, Ambigaipalan et al. (2017) reported the phenolic compounds of pomegranate seed and compared them with that of the extracted juice. They found that the seed of pomegranate had more phenolic compounds than the extracted juice.

\subsection{Banana}

Banana (Musa paradisiaca L., Musaceae) represents one of the most important fruit crops, with a global annual production of more than 50 million tonnes and worldwide production of cooking bananas (plantains) amounts to nearly 30 million tons per year (Schieber et al., 2001b). Peels and bracts are the important by-products of banana fruit. Peels constitute up to $30 \%$ of the ripe fruit. Dopamine (Figure 22) is the only major phenolic constituent in banana peel and it is reported to occur in high concentration $(700 \mu \mathrm{g} / \mathrm{g}$ fresh weight) in banana peel compared to pulp (8 $\mu \mathrm{g} / \mathrm{g}$ fresh weight) (Wacker et al., 1958). Vu et al. (2019) found optimal condition to extract phenolic compounds from banana peel using microwave. The conditions employed were $\mathrm{pH} 1$ of water, $2: 100 \mathrm{~g} / \mathrm{mL}$ (sample to solvent ratio), 6 min irradiation, and $960 \mathrm{~W}$.

Kanazawa and Sakakibara (2000) showed that dopamine levels ranged from $80-560 \mathrm{mg}$ per $100 \mathrm{~g}$ in peel and $2.5-10 \mathrm{mg}$ in pulp, even in ripened bananas ready to eat. Their study revealed that dopamine had a faster radical-scavenging rate than catechin and was similar to gallocatechin gallate. In addition, they reported the presence of other phenolic constituents in peels include flavanone glycoside naringin and flavonol glycoside rutin. Phenolic constituent of banana peel varies at different ripening stages (classification defines eight stages by colour score: all green, light green, half-green, half-yellow, green chip, full yellow, star, and duffel) (Kanazawa and Sakakibara, 2000).

Anthocyanin pigments in banana bracts could be utilized as natural food colourants. Most bananas have red, purple or violet bracts although a few are cyanic - green or yellow. The variation in bract colour is correlated with the composition of the anthocyanins present, which is distinctive of species and subspecies (Simmonds, 1962). Bracts proved to be a good and abundant source of anthocyanins of attractive appearance, as well as being a useful tool in anthocyanin identification since all six most common anthocyani- dins (delphinidin, cyanidin, pelargonidin, peonidin, petunidin and malvidin) are present (Pazmino-Duran et al., 2001) (Figure 23).

\subsection{Longan}

Gallic and ellagic acids are the major phenolic constituents in longan seeds (Soong and Barlow, 2006). Rangkadilok et al. (2007) examined the antioxidant activities of longan seed and pulp extracts by using the scavenging activity of 2,2-diphenyl-1-picrylhydrazyl (DPPH). Their results showed that the polyphenols in longan seed extracts, such as corilagin, gallic acid, and ellagic acid, exhibited the same scavenging effect as DPPH and superoxide radicals in Japanese green tea extracts. Sun et al. (2007) identified two representative polyphenolic compounds, 4-O-methylgallic acid and (-)-epicatechin, by NMR and ESI-MS analyses in longan pericarp. In this study, when comparing reducing power and DPPH hydroxyl radical and superoxide radical-scavenging activities, it could be found that 4-O-methylgallic acid and (-)-epicatechin possessed antioxidant properties and that 4-O-methylgallic acid exhibited stronger antioxidant capability than (-)-epicatechin. Bai et al. (2019) extracted phenolic compounds from longan pericarp and identified protocatechuic acid, isoscopoletin, quercetin, ellagic acid, corilagin, and proanthocyanidins $\mathrm{C} 1$.

\section{By-products of vegetables}

\subsection{Onions}

Onion (Allium cepa L.), one of the oldest vegetable known to humankind, is found in a large number of recipes and nowadays available in fresh, frozen, canned, pickled, powdered, chopped, and dehydrated form. The major by-products resulting from industrial peeling of onion bulbs are brown skin, the outer two fleshy leaves and the top and bottom bulbs. Onions serve as a major dietary source of flavonoids, particularly rich in quercetin glycosides (Hertog et al., 1992; Waldron, 2001). The major flavonoids of mature onion bulbs are quercetin 3,4- $O$-diglucoside and quercetin 4-O-monoglucoside, accounting for more than $85 \%$ of the total flavonoids (Price and Rhodes, 1997). Since quercetin from onions is rapidly absorbed and slowly eliminated, it could contribute significantly to antioxidant defense (Hollman et al., 1997).

The major by-product of onion is dry peel (outer layer), which contain large amounts of quercetin, quercetin glycoside (Figure 
<smiles></smiles>

Gallagic acid

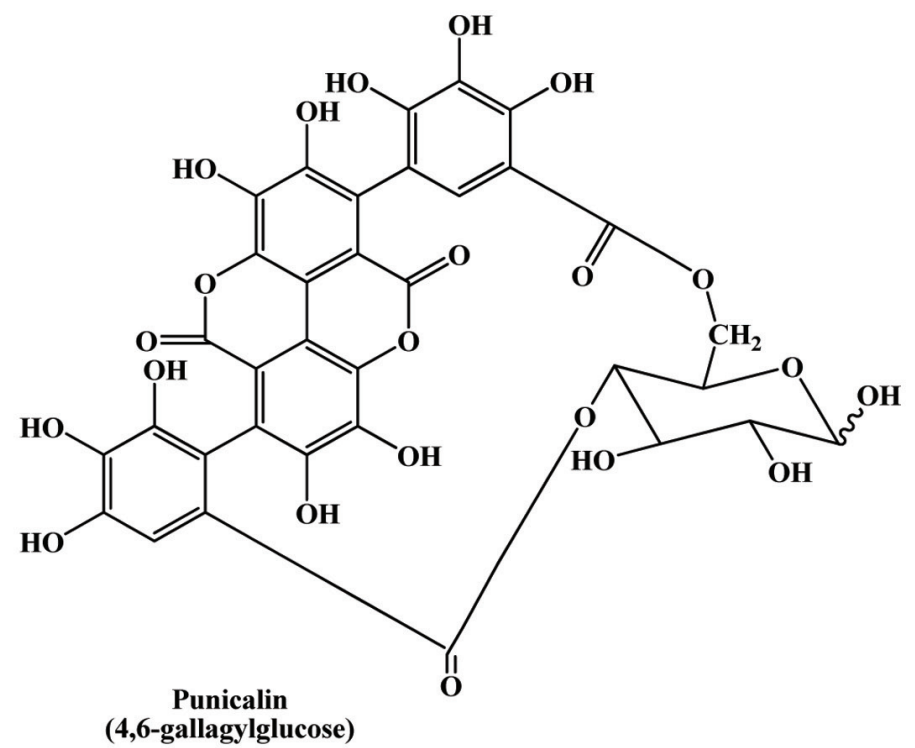

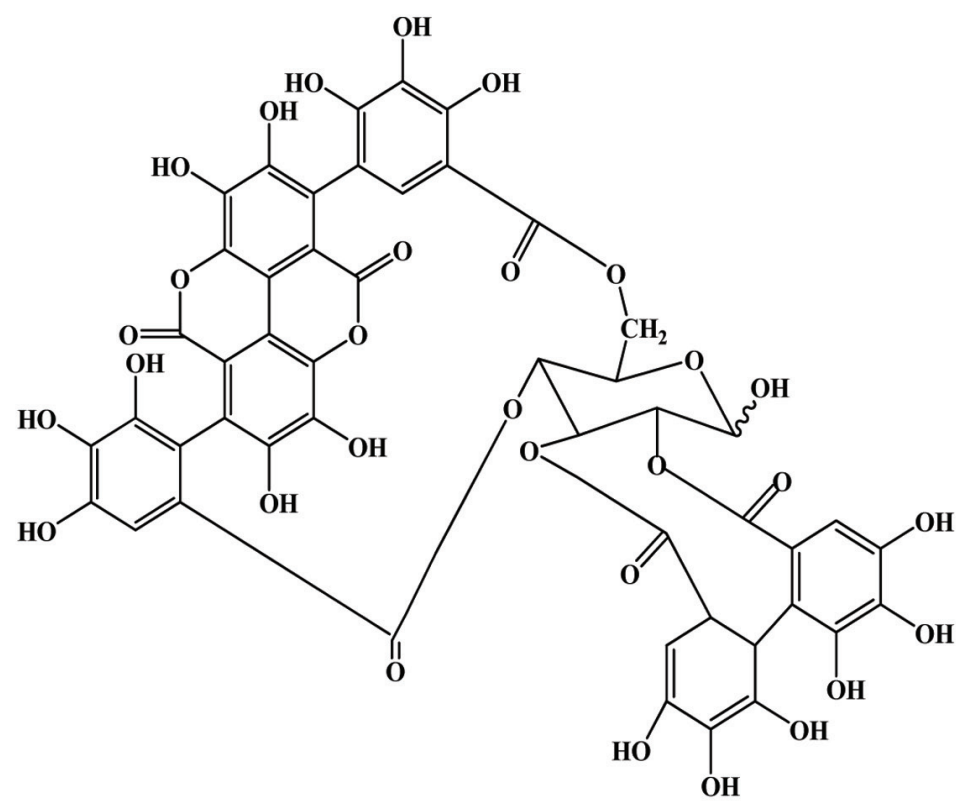

Punicalagin

(2,3-hexahydroxydiphenoyl-4,6-gallagylglucose)

Figure 21. Major phenolic compounds identified in pomegranate peel. Source: Adapted from Seeram et al., (2005).

24) and their oxidative product, which are effective antioxidants against the lethal effect of oxidative stress (Gülsen et al., 2007; Prakash et al., 2007).

In addition to these quercetin compounds, dry onion peels represents kaempferol, ferulic, gallic and protocatechuic acids (Singh et al., 2009). The study of Velioglu et al. (1998) showed that total phenolic content of red onion scale was $105.5 \mathrm{~g} / \mathrm{kg}$ dry matter.

Singh et al. (2009) investigated the different solvent extracts/ fractions (dichloromethane extract and diethyl ether, ethyl acetate, and n-butanol fractions of ethanolic extract) of red onion dry peel and found that ethyl acetate fraction showed high total content of phenolics (385 mg gallic acid equivalents/g) and flavonoids (165 $\mathrm{mg}$ quercetin equivalents $/ \mathrm{g}$ ). Further, ethyl acetate fraction of onion dry peel demonstrated high antioxidant activity, free radical scavenging activity and reducing power compared to other fractions. In another study, the outer layer of red onion showed the highest antioxidants and antioxidant activities than the purple, white, and green varieties of onion, as determined by in vitro antioxidant and free radical scavenging activities (Prakash et al., 2007). In a recent study, Campone et al. (2018) optimized the extraction of phenolic compounds from brown onion. They compared ultrasound-assisted extraction and supercritical carbon dioxide/cosolvent extraction and found that phenolics from the latter method showed better antioxidant activity. Therefore, they suggested using the supercritical carbon dioxide/cosolvent extraction, which is considered as green method, in industrial scale in order to maximize extraction effi- 
<smiles>NCCc1ccc(O)c(O)c1</smiles>

Figure 22. Chemical structure of dopamine.

ciency of phenolic compounds.

\subsection{Tomato}

Tomato is a versatile vegetable that is consumed fresh as well as in the form of processed products. While most tomatoes produced worldwide are used in the production of tomato paste, an ingredient in different processed tomato products such as ketchup, sauces, and soups, a significant number of tomatoes are consumed fresh (Toor and Savage, 2005). However, some consumers remove the skin and seeds of tomatoes before eating them raw, while some fresh tomatoes are cooked with or without the skin and seeds. Approximately one-third of the total weight of tomatoes in the form of skin and seeds is discarded during processing of tomatoes into products like paste, salsa, and sauces (Toor and Savage, 2005). Since tomato is a reservoir of diverse antioxidant molecules, it can provide a significant proportion of the total antioxidants in the diet. These bioactive molecules are largely in the form of carotenes (lycopene) and phenolic compounds (flavonoids and phenolic ac-<smiles>[R10]Oc1ccc(-c2oc3cc(O)cc(O)c3c(=O)c2O[R])cc1O</smiles>

Figure 24. A molecular structure of quercetin and glucosides.

ids) (Beacher, 1998; George et al., 2004; Martínez-Valverde et al., 2002; Toor and Savage, 2005).

Tomato skin contains high levels of phenolic compound compared to pulp and seed (Benakmoum et al., 2008; George et al., 2004; Toor and Savage, 2005). Majority of the flavonols in tomatoes are present in the skin (83\%) (Stewart et al., 2000).

However, phenolic content vary according to fruit variety, size, country of origin (Stewart et al., 2000) agronomical and seasonal factors (Martínez-Valverde et al., 2002). George et al. (2004) in-<smiles></smiles>

\begin{tabular}{llll}
\hline Anthocyanidin & $\mathrm{R} 1$ & $\mathrm{R} 2$ & Colour \\
\hline Cyanidin & $\mathrm{OH}$ & $\mathrm{H}$ & Red \\
Peonidin & $\mathrm{OCH}_{3}$ & $\mathrm{H}$ & Bluish-purple \\
Pelargonidin & $\mathrm{H}$ & $\mathrm{H}$ & Orange-red \\
Malvidin & $\mathrm{OCH}_{3}$ & $\mathrm{OCH}_{3}$ & Purple \\
Delphinidin & $\mathrm{OH}$ & $\mathrm{OH}$ & Purple \\
Petunidin & $\mathrm{OCH}_{3}$ & $\mathrm{OH}$ & Purple \\
\hline
\end{tabular}

Figure 23. Basic structure of anthocyanidins and most common anthocyanidins of banana bracts. Source: Adapted from Pazmino-Duran et al., (2001). 
<smiles>O=C(O)C1(O)CC(O)C(O)C(O)C1</smiles>

Quinic acid<smiles>O=C(O)/C=C/c1ccc(O)c(O)c1</smiles>

Caffeic acid (1,3,4,5-tetrahydroxycyclohexanecarboxylic acid)<smiles>CC(C)C(C)C(=O)O[As]1CC(O)(C(=O)O)CC(O)C1O</smiles>

Chlorogenic acid (5-O-caffeoylquinic acid)

Cryptochlorogenic acid $=(4-\mathrm{O}$-caffeoylquinic acid $)$ Neochlorogenic acid $=(3-\mathrm{O}-$ caffeoylquinic acid $)$ Isochlorogenic acid 'a' $=(4,5$-di-O-caffeoylquinic acid $)$ Isochlorogenic acid ' $b$ ' $=(3,5$-di-O-caffeoylquinic acid $)$

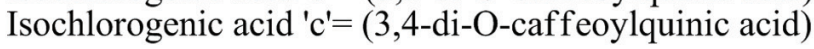

Figure 25. Chlorogenic acid isomers in potato peel. Source: Adapted from Friedman, (1997).

vestigated the different field grown tomato genotypes and found that total phenolic content of peel and pulp ranged from 10-40 and 9-27 mg (catechin equivalents) per 100 gram fresh matter respectively. A similar observation has been made by Toor and Savage (2005) who reported that skin and seeds of tomato contributed $53 \%$ to the total phenolics and $52 \%$ to the total flavonoids in their analysis on three different varieties. In this study, they found that the hydrophilic phenolics in the skin of three cultivars ranged from 26.9 to $30.3 \mathrm{mg}$ (gallic acid equivalents)/100 $\mathrm{g}$ and lipophilic phenolics contributed $14-17 \%$ of the total phenolic content. Furthermore, their study proved that seed fraction of tomatoes as an important reservoir of phenolics.

Flavonoid is found, primarily as conjugates, quercetin and kaempferol and the main conjugate has been reported as rutin (quercetin 3-rhamnosylglucoside) (Crozier et al., 1997a; Crozier et al., 1997b; Stewart et al., 2000; Woldedecke and Herrmann, 1974). In addition, naringenin and hydroxycinnamic acids, such as caffeic, chlorogenic, ferulic and $p$-coumaric acids have been identified in tomato peel and purée. Martínez-Valverde et al. (2002) measured the content of major flavonoid aglycones, and hydroxycinnamic acids in commercial varieties of tomato grown in Spain and found that most abundant hydroxycinnamic acid was chlorogenic acid, with values ranging from 14 to $32 \mathrm{mg} / \mathrm{kg}$ fresh weight, followed by caffeic acid, while $p$-coumaric and ferulic acids showed similar concentrations lower than $5 \mathrm{mg} / \mathrm{kg}$. In the same study they showed that quercetin, the most abundant flavonoid, was found in concentrations ranging between 7.19 and $43.59 \mathrm{mg} / \mathrm{kg}$ fresh weight, while naringenin levels were lower than $12.55 \mathrm{mg}$ per $\mathrm{kg}$. However, in other works the compounds reported to be predominant on the tomato peel and purée were rutin, quercetin and chalconaringenin (Arabbi et al., 2004; Beecher, 1998; Benakmoum et al., 2008;). A study of Stewart et al. (2000) showed that the flavonol contents of tomato fruits independent with their anthocyanin contents. Palomo et al. (2019) recently identified coumaric acid, floridzin, floretin, procyanidin B2, luteolin-7-O-glucoside, kaempferol, and quercetin from tomato pomace extract.

Antioxidant potency of tomato fractions have been reported in numerous work (Martínez-Valverde et al., 2002; George et al., 2004; Toor and Savage, 2005; Benakmoum et al., 2008; Beecher, 1998; Arabbi et al., 2004). Shixian et al. (2005) mentioned that polyphenols contribute to the synergistic effects observed in lycopene. 


\subsection{Potato}

Potato is one of the main vegetables consumed in European and American diets and the production of starch from potatoes produces huge quantities of residual by-products (Pihlanto et al., 2008). Phenolic compounds are distributed mostly between the cortex and skin (peel) tissues of the potato. About $50 \%$ of the phenolic compounds are located in the potato peel and adjoining tissues, while the remainder decrease in concentration from the outside toward the center of potato tubers (Friedman, 1997).

Peels are the major by-products of potato processing. It contains many phenolic compounds, such as chlorogenic, gallic, protocatechuic and caffeic acids some in free form and others that are bound (Albishi et al., 2013a; Al-Weshahy et al., 2013; Kumar et al., 1991; Lyon and Barker, 1984; Malmberg and Theander, 1985; Onyeneho and Hettiarachchy, 1993; Ramamurthy et al., 1992; Rodriguez de Sotillo et al., 1994a; Singh and Rajini, 2008). The different extracts of potato peel displayed phenolic contents ranging from 2.9 to 4.2 $\mathrm{mg} / \mathrm{g}$ potato peel powder (Singh and Rajini 2008). Lisinska and Leszczynski (1987) reported that the largest portion of phenolic acids was chlorogenic acid, a derivative of caffeic acid and quinic acid (Figure 25). In freeze-dried aqueous extract, chlorogenic, caffeic, gallic, and protocatechuic acids, present as 50.3, 41.7, 7.8, and $0.21 \%$, respectively, of the total phenolics (Rodriguez de Sotillo et al., 1994b). Phenolic contents in boiled potato peel were significantly higher than that from boiled whole potato (Mattila and Hellström, 2007; de Ancos et al., 2015).

In another study, Rodriguez de Sotillo et al. (1994a) found that the water reflux method gave the greatest amounts of chlorogenic, gallic, and protocatechuic acid compared to methanolic extract, but gave the lowest amount of caffeic acid. The study of Singh and Rajini (2008) showed higher the amount of caffeic acid and lower amount of chlorogenic acid in ethanolic extract. According to their study the relative composition of the four phenolic acids; caffeic, gallic, protocatechuic, and chlorogenic, were 38.6, 26.5, 18.8, 16\% respectively. Therefore, application of high temperature $\left(100{ }^{\circ} \mathrm{C}\right)$ may degrade the caffeic acid into other compounds.

The antioxidant activity of freeze-dried water extracts of potato peels was comparable to that of butylated hydroxyanisole (Rodriguez de Sotillo et al., 1994b). The extracts displayed species-dependent antibacterial but no mutagenic activity, and concentrations of the glycoalkaloids solanine and chaconine were below toxic threshold levels if peel extracts were added at $200 \mathrm{ppm}$ to a food (Rodriguez de Sotillo et al., 1998). However, methods for the complete separation of steroidal alkaloids from phenolic compounds prior to their use in foodstuff would be desirable to avoid any risk for human health (Rodriguez-Saona et al., 1998, 1999).

\subsection{Red beet}

The pomace from the beetroot juice industry accounting for 15 $30 \%$ of the raw material (Otto and Sulc, 2001) is disposed as feed or manure. Whereas the coloured fraction consisted of betacyanins and betaxanthins, the phenolic portion of the peel showed $l$-tryptophan, $p$-coumaric and ferulic acids, as well as cyclodopa glucoside derivatives (Kujala et al., 2001). Betalains and anthocyanins are mutually exclusive in their natural occurrence, but other flavonoids (e.g., flavonols and flavones) are often produced in betalain-bearing plants (Stafford, 1994). The total phenolics distribution in red beet (Beta vulgaris), root appears to be quite similar to that reported for the potato. Kujala et al. (2001) showed that total phenolics decreased in the order peel (50\%), crown (37\%), and flesh (13\%).
The total phenolic content of beetroot pomace from juice production ranged from 87 to $151 \mathrm{mg}$ gallic acid equivalents per gram dry extract (Peschel et al., 2006). The phenolic compounds found in beet leaves and seeds were syringic acid, caffeic acid, coumaric acid, ferulic acid, vanillic acid, protocatechuic acid, $p$-hydroxybenzoic acid, chlorogenic acid, kaempferol, and apigenin (Pyo et al., 2004; Ninfali and Angelino 2013; de Ancos et al., 2015).

\subsection{Carrot}

Carrot (Daucus carota L.) can be eaten in a variety of ways such as raw carrots, broths, soups, and stews. Grated carrots are used in carrot cakes, as well as carrot puddings. Baby carrots or mini-carrots (after peeling) are a popular ready-to-eat snack food available in many supermarkets. Carrot juice is also widely marketed, especially as a health drink, either stand-alone or blended with fruits and other vegetables. During all these processes large amount of by-products, such as peels and pomace are derived. Peel accounted for only about $11 \%$ of the carrot fresh weight. They contain high contents of beneficial substances, especially bioactive compounds with antioxidant activities (Chantaro et al., (2008); Zhang and Hamauzu, 2004). Carrot peels have been reported to be a rich source of phenolic compounds (Chantaro et al., (2008); Kähkönen et al., 1999; Zhang and Hamauzu, 2004). Zhang and Hamauzu (2004) reported that phenolic composition in different tissues in carrots is similar, but the content of individual phenolic compounds in different tissues decreased in the following order: peel $>$ phloem $>$ xylem. Although carrot peel accounted for only $11.0 \%$ of the amount of the carrot fresh weight, it could provide $54.1 \%$ of the amount of the phenolics in $100 \mathrm{~g}$ fresh weight of carrots.

Chlorogenic acid is the predominant phenolic acid present in carrots along with other hydroxycinnamic acids and its derivatives such as caffeic acid, 3-caffeoylquinic acid, 4-p-coumaroylquinic acid, 3,4-dicaffeoylquinic acid, 3,5-dicaffeoylquinic acid (Zhang and Hamauzu, 2004). The isocoumarins 6-hydroxymellein and 6-methoxymellein have been identified by Harding and Heale (1980). The presence of phenolic compounds in carrot peels under different processing conditions were investigated by Chantaro et al. (2008) and found that the total phenolic content in carrot peels was approximately $1,380 \mathrm{mg}$ (gallic acid equivalents)/100 g dry weight. In another study, Zhang and Hamauzu (2004) showed that total phenolic content in fresh carrot peels was $979 \mathrm{mg}$ (gallic acid equivalents)/100 g dry weight and phenolic content of different tissues of carrot correlated well with antioxidant and radical scavenging ability. Further, its radical scavenging ability was superior to pure chlorogenic acid, vitamin $\mathrm{C}$, and $\beta$-carotene. Kamiloglu et al. (2016) identified several anthocyanidins such as cyanidin3-xylosyl-glucosyl-galactoside, cyanidin-3-xylosyl-galactoside, cyanidin-3-xylosyl-sinapoyl-glucosyl-galactoside, cyanidin-3-xylosyl-feruloyl-glucosyl-galactoside, and cyanidin-3-xylosyl-coumaroyl-glucosyl-galactoside in peels of black carrot. Moreover, phenolic extracts from their peels displayed a high antioxidant potential in total phenolic content, total monomeric anthocyanin content, and total antioxidant capacity. Therefore, the higher level of phenolic compounds in peel could be considered as a promising source of nutraceuticals and other value-added processing industries.

\subsection{Pumpkin}

Pumpkin is a gourd-like squash of the genus Cucurbita and the 
family Cucurbitaceae. Pumpkin seeds, also known as pepitas, are small, flat, green, edible seeds. Most pumpkin seeds are covered by a white husk, and some pumpkin varieties have hull-less seed. Pumpkin is mainly cultivated as a vegetable crop for human consumption and their seeds are a popular snack and a good source of oil. Peels, hull and oil cake meal are the by-products of pumpkin processing industry. In peels, small amounts of vanillic acid, $p$ coumaric acid, and sinapic acid were reported (Schmidtlein and Herrmann 1975). Peričin, et al. (2009) studied the distribution of phenolic acids by high performance liquid chromatography and reported that $p$-hydroxybenzoic acid was the dominant phenolic compound with $52.0 \%$ in oil cake meal and $51.8 \%$ in hulls based on total phenolic acid content $(89 \mathrm{mg} / \mathrm{kg}$ and $158 \mathrm{mg} / \mathrm{kg}$ in dry weight of oil cake meal and hulls, respectively). In this study they isolated and identified protocatechuic, $p$-hydroxybenzoic, vanillic, trans- $p$-coumaric, ferulic, trans-sinapic acids caffeic, syringic and $p$-hydroxybenzaldehyde in hulls and oil cake but caffeic acid in hulls and syringic acid in oil cake were not detected. Furthermore, most of these phenolic acids were present in bound (esterified and insoluble) form; $51.8 \%$ in oil cake and $51.9 \%$ in hulls. Saavedra et al. (2015) studied antioxidant potential of pumpkin byproduct (shell); it showed 2.0-10.6 mg gallic acid equivalents/g dried weight in total phenolic content.

\subsection{Artichoke}

Artichoke (Cynara scolymus L.) is an ancient herbaceous plant, originating from the Mediterranean area, which today is widely cultivated all over the world. Its flower head is eaten as a vegetable and prepared for different value-added products such as salad, jam, concentrate, and canned beverages. Artichoke extract has been widely used as a medicine in many countries for a long time (Zhu et al., 2004). Artichoke-based industry yield residue from both fresh handling, which mainly include solid waste such as bracts, receptacles and stems and industrial canning process, which mainly comprise blanched water and solid wastes.

Caffeic acid and its derivatives are the main phenolic compounds in artichoke head (Lattanzio et al., 1994). Other phenolics, such as flavonoids, apigenin, and luteolin (both glucosides and rutinosides) (Lattanzio and van Sumere, 1987) as well as different cyanidincaffeoylglucoside derivatives (Aubert and Foury, 1981) have been identified. Llorach et al. (2002) analyzed the total phenolic content of blanching water and methanolic and water extract of solid by-products of raw and blanched artichoke and found that methanolic extract yielded more phenolics than water extract. According to their study, total phenolic content of raw and blanched were 15.4 and $24.3 \mathrm{~g} / 100 \mathrm{~g}$ of dry extract (expressed as caffeic acid derivatives) respectively. While blanching, water yielded $11.3 \mathrm{~g}$ of phenolics per $100 \mathrm{ml}$ of blanching water.

Artichoke leaf extract, which is also an important by-product, exhibits antioxidant, anti HIV, liver protective, bile expelling, and lipid lowering properties (Gebhardt, 1997 and 1998; Liorach et al., 2002; McDougall, et al., 1998). Zhu et al. (2004) investigated the antimicrobial constituents from artichoke leaf extract and they isolated and identified eight phenolic compounds, of which four caffeoylquinic acid derivatives, chlorogenic acid, cynarin, 3,5-di- $O$-caffeoylquinic acid, cynarin, and 4,5-di- $O$-caffeoylquinic acid, and the four flavonoids, luteolin-7-rutinoside, cynaroside, apigenin-7-rutinoside and apigenin-7- $O-\beta$-D-glucopyranoside, respectively. Detailed investigation of Sanchez-Rabaneda et al. (2003) on phenolic substances in the artichoke by-product set out the presence of 45 phenolic compounds. Ruiz-Cano et al. (2014) reported a high antioxidant potential of artichoke byproduct from industrial canning processing; namely, total phenolic content (153$729 \mu \mathrm{mol}$ gallic acid equivalents/g dried material), total flavonoid content (6.9-19.2 $\mu \mathrm{mol}$ quercetin equivalents/g dried material), and antioxidant activity (85-234 $\mu \mathrm{mol}$ ascorbic acid equivalents/g dried material).

\subsection{Brassica crops}

Cauliflower, cabbage, and broccoli are the main Brassica crops. Significant amount of antioxidants such as ascorbic acid, phenolic compounds and tocopherols has been reported in brassica crops (Kim et al., 2004; Singh et al., 2006; Wennberg et al., 2004). During the processing of these vegetables about $40 \%$ of outer leaves and core of cabbages are discarded and treated as wastes (Nilnakara et al., 2009) and may only be used for fertilizer or animal feed. Regarding the by-product proportion, leaves constitute about 50\% of the total; the rest is mainly stem (Llorach et al., 2003).

Wijngaard et al. (2009) measured the total phenolic content of freeze dried vegetable by-products (methanolic extract) by Folin Ciocalteu Reagent (FCR). They found that total phenolic content of white cabbage cut-offs, cauliflower cut-offs, and broccoli stems were 341, 402 and $494 \mathrm{mg}$ (gallic acid equivalents) per 100 g dry weight respectively. According to the study of Nilnakara et al. (2009) the total phenolic content of fresh and blanched (2 $\mathrm{min} /$ boil water) white cabbage by-product were 571 and $349 \mathrm{mg}$ (gallic acid equivalents)/100 g of dry matter. Blanching caused about $39 \%$ loss of total phenolic content. Similar observation has been made by Ismail et al. (2004) who reported 20\% loss of total phenolic content in cabbage (Brassica oleracae) after 1 min of blanching in boiling water. Total phenolic content drop after drying and at higher drying temperatures loss is higher (Nilnakara et al., 2009).

Cauliflower by-products (Brassica oleracea L. var. botrytis) mainly consist of leaves and, in fewer amounts, stems (TomásBarberán et al., 2004). Llorach et al. (2003) analysed the phenolic profile of cauliflower by-products by HPLC in water and ethanolic extracts and revealed the presence of both flavonoids and hydroxycinnamic acids (caffeic acid and sinapic acid). Different combinations of flavonols such as kaemferol and quercetin with sinapic acid and glucose were reported as the main phenolic compounds in both ethanol and water extracts. These major compounds have been identified as kaempferol-3-O-sophoroside-7-O-glucoside and its sinapoyl derivative kaempferol-3-O-(sinapoylsophoroside)7-O-glucoside. In addition, neochlorogenic acid, quercetin3-O-sophoroside-7-O-glucoside, kaempferol-3- $O$-sophoroside, 1,2-disinapoylgentiobiose, and 1,2,2-trisinapoylgentiobiose have been detected. The edible part of cauliflower is rather poor in phenolic compounds, consist only hydroxycinnamic acid such as caffeic, sinapic, and ferulic acids but the overall concentration of these compounds $(0.18 \mathrm{~g} / \mathrm{kg}$ fresh weight $)$ was 2 -fold higher than that found in the cauliflower by-products $(0.094 \mathrm{~g} / \mathrm{kg}$ fresh weight $)$ (Llorach et al., 2003). However, the flavonoids concentration in cauliflower by-products was much higher than that found in the edible parts where only trace amounts were detected (Llorach et al., 2003). Furthermore, the study of Llorach et al. (2003) revealed that the cauliflower by-products presented 3-fold higher flavonols content than other Brassica species and the extracts from cauliflower by-products had a good scavenging activity against both DPPH and ABTS radicals. Drabińska et al. (2018) investigated the antioxidant capacity of phenolics in broccoli by-products, and their phenolic extracts and reported total phenolic content of 9.5 $\mathrm{mg}$ gallic acid equivalents (GAE)/g of dried material and ABTS 
radical cation activity of $99.8 \mu \mathrm{mol}$ trolox equivalents (TE)/g of dried material.

\subsection{Lettuce and chicory}

Lettuce and escarole, belonging to the Asteraceae family, are the most popular vegetables in salads which are consumed in increasing amounts due to their perception as being "healthier" foods (Dupont et al., 2000). Lettuce has two methods of commercialization, one as whole lettuce heads and the other as fresh-cut product. Nowadays, there has been a great development of the fresh-cut vegetable industry, fresh-cut lettuce being one of the most important products. The packinghouses dealing with vegetables produce large amounts of wastes and residues (leaves, stems, etc.). Sometimes these by-products can reach $50 \%$ of the harvested material as in lettuce production (Llorach et al., 2004).

Llorach et al. (2004) investigated the by-products (mainly external leaves) from lettuce (Lactuca sativa L.) varieties (Romaine, Iceberg and Baby) and one of chicory (Cichorium endivia L.) variety "escarole" to evaluate their polyphenolic content as well as their antioxidant capacity. In this study they found that phenolic profile of lettuce by-products is composed by hydroxycinnamic acids (both caffeoylquinic and caffeoyltartaric acid derivatives) and flavonoids (both flavones and flavonols). The main hydroxycinnamic acid derivative identified was dicaffeoyltartaric acid (chicoric acid) followed by chlorogenic acid (5-Ocaffeoylquinic acid). In addition different 45 isomers of isochlorogenic acid (3,5-O-dicaffeoylquinic acid) were identified. In case of flavonoids, flavone Luteolin-7- $O$-glucuronide was identified, and regarding the quercetin derivatives quercetin-3-O-glucoside, quercetin 3-O-glucuronide and quercetin 3-O-(6-O-malonyl)glucoside have been identified. Regarding chicory by-products the HPLC analyses of raw extracts showed a kaempferol 3-Oglucoside as the main flavonol and this compound has already been reported in chicory. Lettuce by-products have shown a interesting antioxidant capacity both free radical scavenging activity and capacity to reduce Fe(III) to Fe(II) (Llorach et al., 2004). Compared to other vegetables, the phenolic content of lettuce is relatively low, however due to the high consumption of lettuce, it should be considered as major phenolic source (de Ancos et al., 2015).

\subsection{Asparagus}

Asparagus is a plant food highly appreciated by consumer due its organoleptic, nutritional and functional properties, and it is one of the thirty most consumed vegetables in the world (Ulrich et al., 2001). Prior to the commercialisation of both fresh and processed asparagus, the spears are cut to a determined length, and sometimes they are also peeled. It has been reported that asparagus contains flavonoids (mainly rutin) and other phenolic compounds that possess strong antioxidant properties (Makris and Rossiter, 2001; Vinson et al., 1998). In addition to these phytochemicals, asparagus by-products represent a good source of compounds, such as hydroxycinnamic acids derivatives (Rodríguez et al., 2004). Asparagus by-products also serve as a good source of dietary fiber as they are linked to phenolic compounds and form insoluble-bound phenolics. Jaramillo-Carmona et al. (2019) used hydrothermal treatment to release oligosaccharides and phenolic compounds from asparagus by-products and found that the antioxidant activity was increased in a temperature-dependent manner.

\section{By-products of tree nuts}

Tree nuts and their by-products such as skin or testa, hard shell, green leafy cover, hull and leaf are rich sources of phytochemicals that posses multifunctional properties such as antioxidant and free radical scavenging activity (Alasalvar and Shahidi 2009; Chang et al., 2016).

\subsection{Almond}

Almond, scientifically known as Prunus dulcis belongs to the Rosaceae family. It is the number one tree nut produced on a global basis (Alasalvar and Shahidi, 2009). Skin (seed coating), brown hull and green shell cover (mesocarp) are the by-products derived from the industrial processing of almond. The production of almond hulls, which are mainly used in livestock feed, is estimated to exceed 6 million tonnes annually (Takeoka et al., 2000) and this amount reached 4.262 billion pounds (Annual Report of Almond Board of Califonials Almond Almanac, 2017). Almond hulls and shells possess powerful free radical scavenging capacities (Frison-Norrie and Sporns, 2002a; Moosavi et al., 2015) and these activities could be due to the presence of triterpenoids, flavonoids and phenolic acids, or even polysaccharides (Sfahlan et al., 2009; Dammak et al., 2018)

Phenolic compounds such as catechin, protocatechuic acid, chlorogenic acid (5-O-caffeoylquinic acid), cryptochlorogenic acid (4-O-caffeoylquinic acid), neochlorogenic acid (3-O-caffeoylquinic acid) and a prenylated benzoic acid derivative (2-prenyl-4$O$ - $\beta$-D-glucopyranosyloxy-4-hydroxybenzoic acid) have been identified in almond hulls (Sang et al., 2002a) (Figure 26). Green shell cover also serves as a better source of phenolic acids such as caffeic acid, $p$-coumaric, ferulic and sinapic acid (approximately $0.1 \%$ of the extract) compared with whole seed and brown skin extracts. It was found that caffeic acid was the predominant phenolic acid and mostly present in the bound form, but was only in trace amounts in the free form in almond shell. The total amounts of the free phenolic acids in brown skin and green shell cover extracts were 16.28 and $13.99 \mu \mathrm{g} / \mathrm{g}$ extract and the total esterified phenolic acids in the whole seed, brown skin, and green shell cover extracts were 40.34, 279.55, and $967.10 \mathrm{mg} / \mathrm{g}$ extract, respectively (Wijeratne et al., 2006). The comparative study of phenolic contents in different genotype of almond hulls and shells showed that the total phenolic content in hulls was higher than that of the shells (Sfahlan et al., 2009). Almond skin contains flavonoid and phenolic acids include protocatechuic, vanillic, $p$-hydroxybenzoic acid, ferulic and sinapic acid (Milbury et al., 2006; Monagas et al., 2007; Sang et al., 2002b; Wijeratne et al., 2006) (Figures 27 and 28).

Brieskon and Betz (1998) first identified the flavanol monomers $(+)$-catechin and (-)-epicatechin as well as dimmers constituted by these units (procyanidins B1, B3, B4) but not by trihydroxylated ones (i.e. gallocatechin and epigallocatechin). Lazarus et al. (1999) confirmed the presence of B-type procyanidins, at the same time eliminating the presence of A-type procyanidins in almond skins. Later, Amarowicz et al. (2005) reported the presence of procyanidins B2 and B3 as well as delphinidin and cyanidin after n-butanol$\mathrm{HCl}$ hydrolysis. In case of flavonols, the 3-O-glucosides, galactosides, and rutinosides of quercetin, kaempferol, and isorhamnetin, as well as their corresponding aglycones, have been identified in almond skins (Frison-Norrie and Sporns, 2002a, 2002b; Milbury et al., 2006; Sang et al., 2002b; Wijeratne et al., 2006;). Milbury et al. (2006) reported the presence of morin and dihydrokaempferol in almond skin. In addition, flavanones naringenin-7-O-glucoside, 
<smiles>COc1ccc(C(=O)O)cc1CC=C(C)C</smiles>

A<smiles>O=C(/C=C/c1ccc(O)c(O)c1)OC1CC(O)(C(=O)O)CC(O)C1(O)O</smiles>

B

\section{A 3-Prenyl-4-O- $\beta$-D-glucopyranosyloxy-4-hydroxylbenzoic acid \\ B 1. Chlorogenic acid (5-O-caffeoylquinic acid) \\ 2. Cryptochlorogenic acid (4-O-caffeoylquinic acid) \\ 3. Neochlorogenic acid (3-O-caffeoylquinic acid)}

Figure 26. Phenolic compounds in almond hulls. Source: Adapted from Sang et al., (2002a).

eriodictyol-7- $O$-glucoside, and eriodictyol-7- $O$-galactoside and their corresponding aglycones have been identified in almond skin (Milbury et al., 2006; Sang et al., 2002b). Monagas et al. (2007) identified thirty three compounds corresponding to flavanols, flavonols, dihydroflavonols, and flavanones, and other non-flavonoid compounds in almond skin by HPLC-DAD/ESI-MS technique and reported that flavanols and flavonol glycosides were the most abundant phenolic compounds in almond skins, representing up to $38-57 \%$ and $14-35 \%$ of the total quantified phenolics, respectively. In relation to flavonol glycosides, rutinosides and in particular isorhamnetin-3-O-rutinoside (narcissin) were the most abundant forms. Valdés et al (2015) identified a variety of phenolics such as catechin, quercetin-3-O-rutinoside, kaempferol, and their derivatives in almond skin byproducts.

Kornsteiner et al. (2006) found the distribution of almond phenolics to be predominantly within the "seed skin" (80\%). A similar observation has been made by Milbury et al. (2006) who reported that about $60 \%$ of almond phenolics were present in the skin and eight of the 19 flavonoids and three phenolic acids determined were found exclusively in the skin, on average, $94 \%$ of individual flavonoids originated from the skin in their study on California almond skins and kernels among the principal almond varieties. Their study revealed that the differences in total phenolics between almond varieties were primarily due to differences in the content of skin.

Antioxidant potential of almond by-products have been reported in several studies (Sfahlan et al., 2009; Siriwardhana and Shahidi, 2002; Subhashinee et al., 2006; Wijeratne et al., 2006). Brown skin and green shell cover extract possess more potent antioxidant capacity and free radical scavenging activity than that of the whole seed extract (Siriwardhana and Shahidi, 2002). Frison-Norrie and Sporns (2002) also reported that the hulls and shells of almond possess powerful free radical scavenging capacities. Garrido et al. (2007) studied the phenolic contents in the extraction of three byproducts (skin, shell, and hull) of almonds using different solvent/ mixtures. They found that the extracting solution methanol/ $\mathrm{HCl}$ $(1,000: 1, \mathrm{v} / \mathrm{v})$ was the most effective for the almond skin and shell for antioxidant extraction and the acetone/water (50:50, v/v) mixture exhibited the highest content of polyphenols and proanthocyanidins. In this study they reported that the antioxidant capacity of these by products especially the skin and shell is similar to that of grape by-products, which are currently used in the development of antioxidant products. A similar observation has been made by Sfahlan et al. (2009) who reported that methanolic extract of hull and shell showed a strong antioxidant activity. These studies indicate that antioxidant activity of hull and shell would be helpful in preventing or slowing the progress of various oxidative stress-related diseases. Similarly, Meshkini (2016) reported the protective effect on oxidative damage and membrane protein degradation that are caused in human erythrocytes by hydrogen peroxide.

\subsection{Hazelnut}

Hazelnut (Corylus avellana L.), which belongs to the family Betulaceae, is one of the most popular tree nuts on a worldwide basis and ranks fifth in tree nut production after almond, walnut, cashew, and pistachio in 2017 (Statistical Yearbook of INDFC, 2017). Figure 29 shows the hazelnut fruit and its by-products. The hazelnut green leafy covers, occasionally together with hazelnut tree leaves, are mechanically removed from hazelnut hard shells soon after harvesting. The hazelnut hard shell, containing a kernel, is the nut of commerce. After the hazelnut hard shell has been cracked, the hazelnut kernel may be consumed raw (with skin) or preferably roasted (without skin). In brief, hazelnut skin, hazelnut hard shell, and hazelnut green leafy cover as well as hazelnut tree leaf are by-products of roasting, cracking, shelling/hulling, and harvesting processes, respectively.

Shahidi et al. (2007) evaluated the antioxidant efficacies of ethanolic extracts of hazelnut kernel and its by-products and found that the phenolic contents (milligrams of catechin equivalents (CE) per gram) of hazelnut kernel, hazelnut skin, hazelnut hard shell, hazelnut green leafy cover, and hazelnut tree leaf were 13.7, 577.7, $214.1,127.3$, and 134.7 respectively. In this study, they tentatively identified a total of five phenolic acids one of which was a hydroxylated derivative of benzoic acid (gallic acid) and four of which were cinnamic acid derivatives (caffeic acid, $p$-coumaric acid, ferulic acid, and sinapic acid). In addition, there were several unknown compounds in both free and esterified phenolic acids. The same number, but different concentrations, of phenolic acids were also identified in previous study on hazelnut kernel and hazelnut green leafy cover (Alasalvar et al., 2006). The order of total phenolic acid concentration in hazelnut kernel and its by-products is as follows: hazelnut hard shell $>$ hazelnut green leafy cover $>$ hazelnut tree leaf $>$ hazelnut skin $>$ hazelnut kernel. In another study, Amaral et al. (2005) identified and quantified four phenolic acids, namely, 3-caffeoylquinic acid, 5-caffeoylquinic acid, caffeoyltar- 
<smiles>COc1cc(-c2oc3cc(O)cc(O)c3c(=O)c2O)ccc1O</smiles><smiles>O=c1c(O)c(-c2ccc(O)cc2)oc2cc(O)cc(O)c12</smiles><smiles>COc1cc(-c2oc3cc(O)cc(O)c3c(=O)c2OC)ccc1O</smiles><smiles>CCOc1c(-c2ccc(O)c(OC)c2)oc2cc(O)cc(O)c2c1=O</smiles><smiles>COc1c(-c2ccc(O)cc2)oc2cc(O)cc(O)c2c1=O</smiles><smiles>COc1cc(O)c2c(c1)OC(c1ccc(O)cc1)CC2=O</smiles>

Figure 27. Chemical structure of flavonols, flavonol glycosides and flavonone glycoside identified in almond skin (Sang et al., 2002). 1. Quercetin; 2. Kaempferol; 3. Isorhamnetin; 4. 3-O-methylquercetin 3-O-B-d-glucopyranoside; 5. 3-O-methylquercetin 3-O-B-d-galactopyranoside; 6. 3-O-methylquer-

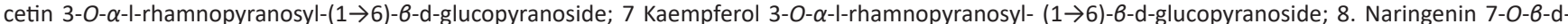
glucopyranoside (prunin).

taric acid, and $p$-coumaroyltartaric acid, in hazelnut leaves from 10 different cultivars grown in Portugal. Recently, 29 phenolics were tentatively identified by HPLC-MS/MS including various derivatives of hydroxylbenzoic acid, hydroxylcinnamic acid as well as other flavanones, flavones, flavanols, and proanthocyanin oligomers (Yuan et al., 2018). The antioxidant capacity of hazelnut by-products shows superiority to hazelnut kernel (Alasalvar et al., 2006; Shahidi et al., 2007). Arcan and Yemenicioğlu (2009) showed that the removal of seed coat reduced almost $36 \%$ of the total antioxidant activity of hazelnuts. Esatbeyoglu et al. (2014) isolated and identified dimeric procyanidins B1-B8 from roasted hazelnut skins.

\subsection{Cashew}

Cashew (Anacardium occidentale) is a tropical tree and one of the most economically important genera in the Anacardiaceae family. Cashew nut shell liquid, cashew nut skin are the important byproducts of cashew nut processing industry. Cashew contains a large quantity of phenolic compounds in a viscous and highly vesicant liquid surrounds the edible kernel known as cashew nut shell 
<smiles>Oc1cc(O)c2c(c1)O[C@H](c1ccc(O)c(O)c1)[C@H](O)C2</smiles>

$(+)$-Catechin<smiles>Oc1cc(O)c2c(c1)O[C@H](c1ccc(O)c(O)c1)[C@H](O)C2</smiles>

(-)-Epicatechin<smiles>O=C(O)c1ccc(O)cc1</smiles><smiles>O=C(O)c1ccc(O)c(O)c1</smiles><smiles>COc1cc(C(=O)O)ccc1O</smiles><smiles>COc1cc(/C=C/C(=O)O)ccc1O</smiles><smiles>COc1cc(/C=C/C(=O)O)cc(OC)c1O</smiles>

Ferulic acid

Sinapic acid<smiles>CCCCOC(O)C1Oc2cc(O)cc(O)c2C(=O)C1c1ccc(O)cc1O</smiles>

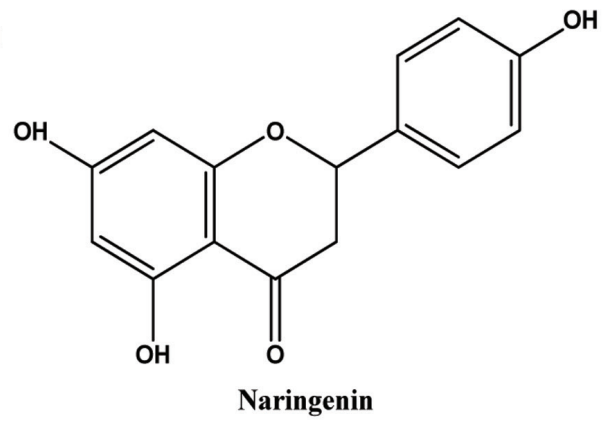

Figure 28. Structures of flavan-3-ols, phenolic acids and flavanones identified in almond skin. Source: Adapted from Shahidi et al., (2009).

liquid (CNSL) (Kubo et al., 1993a; Setianto et al., 2008; Trevisan et al., 2006). The potential annual availability of this material, which accounts for about $32 \%$ of the shell, is enormous (Trevisan et al., 2006).

Trevisan et al. (2006) analyzed the content of anacardic acids, cardanols and cardols in cashew apple, nut (raw and roasted) and cashew nut shell liquid (CNSL) and found that higher amounts $(353.6 \mathrm{~g} / \mathrm{kg})$ of the major alkyl phenols, anacardic acids were in CNSL followed by cashew fibre $(6.1 \mathrm{~g} / \mathrm{kg})$ while the lowest $(0.65$ $\mathrm{g} / \mathrm{kg}$ ) amounts were in roasted cashew nut. Earlier, Tyman et al. (1984) detected 2-methyl cardol on the analysis of natural CNSL by high performance liquid chromatography (HPLC). Kubo et al. (1993a) reported that the anacardic acids, cardols, and cardanols are the principal phenolic components in cashew nut shell liquid (Figure 30). Approximately $50 \mathrm{~mol} \%$ anacardic acids, $29 \mathrm{~mol} \%$ cardols, and $21 \mathrm{~mol} \%$ cardanols including mono-, di-, and triene constituents are present in cashew nut shell liquid (Setianto et al., 2008). In previous study of Trevisan et al. (2006) showed that cardanols were $30 \%$ and cardols were $20 \%$ while Shobha et al. (1994) reported that approximately $70 \%$ of CNSL were anacardic acids and $25 \%$ cardols. Cardanol of CNSL, extracted from cashew nut shells, can be used to produce 3-vinylphenol, which is an important intermediate for synthesizing different drugs such as norfenefrine, rac-phenylephrine, etilefrine, and fenoprofene (Shi 


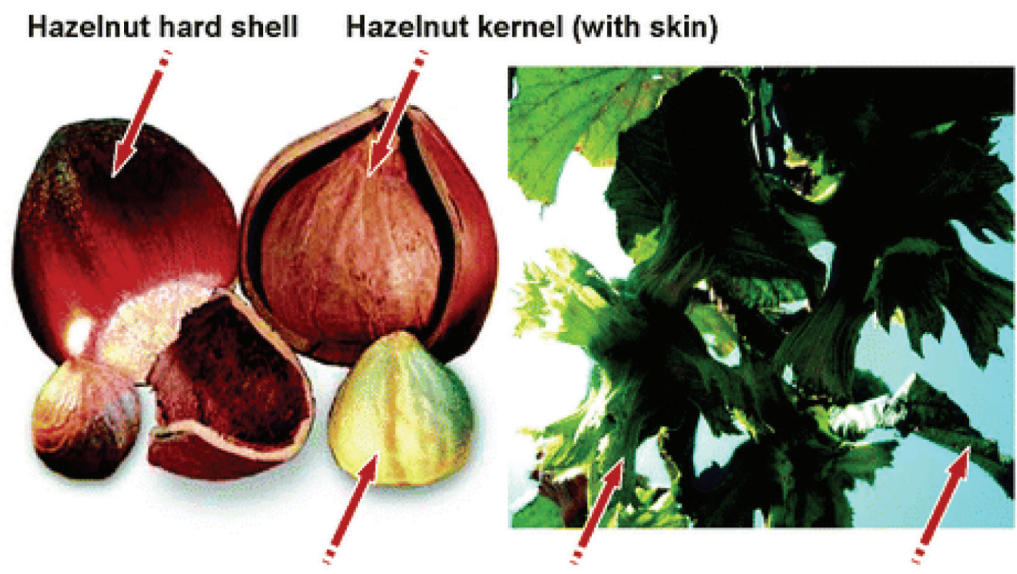

Hazelnut kernel (without skin) Hazelnut green leafy cover Hazelnut tree leaf

Figure 29. Hazelnut kernel and hazelnut by-products (Shahidi et al., 2007).

et al., 2019). Trevisan et al. (2006) reported that hexane extracts $(10 \mathrm{mg} / \mathrm{ml})$ of CNSL displayed significant antioxidant capacity followed by the hexane extract of cashew fibre and apple and antioxidant capacity correlated significantly with the concentration of alkyl phenols, in particularly the concentration of anacardic acids in the extracts. Anacardic acids show pharmacological (Laurens et al., 1997; Toyomizu et al., 1999) antioxidant (Kubo et al., 2006), antibacterial (Hamad and Mubofu, 2015), anti-inflammatory (Yasutake et al., 2013), and antitumor activities (Kubo et al., 1993b; Gény et al., 2016).

Cashew nut skin, an important by-product of cashew processing industry, is a natural source of phenolic compounds. Epicatechin is the major phenolic compound present in the cashew nut skin (Kamath and Rajini, 2007). Earlier studies have shown that cashew nut testa is a good source of hydrolysable tannins (Pillai et al., 1963). More than $40 \%$ of the total polyphenol in the testa is reported to be constituted by $(+)$ catechin and (-) epicatechin (Mathew and Parpia, 1970). Kamath and Rajini (2007) found that the yield of ethanolic extract of the skin powder was quite high $(0.45 \mathrm{~g} / \mathrm{g}$ powder $)$ with a total phenolic content of $243 \mathrm{mg} / \mathrm{g}$ extract. It is indicated that the cashew nut skin/testa contains high amount of phenolics. In the same study, they reported that the ethanolic extract of cashew nut skin has significant antioxidant activity in various antioxidant assay systems. Maia et al. (2015) reported that phenolics such as anacardic acid, cardol, cardanol, 2-methylcardol in cashew nut shell liquid (CNSL), a byproduct in the processing of cashew nuts, inhibits the oxidation of lipids. The presence of the potent bioactive phenolic compounds in the skins of cashew nut could be of interest to both food and pharmaceutical industries, where it could be employed as an economical source of natural antioxidants. Cashew fibre, cashew leaf and bark also got considerable attention due to their role in biological activities.

\subsection{Pistachio}

The pistachio nut is one of the principal tree nuts of the Middle Eastern region and is a significant agricultural export of some of the countries in this area (Vahabzadeh et al., 2004). The pistachio fruit itself consists of a single seed (kernel), encased by a thin soft and edible seed coat (testa), enclosed by a hard inedible shell (endocarp), which is further surrounded by the fleshy hull (meso- carp and epicarp), which is also an inedible. Pistachios are unique among tree nuts in that their endocarp (shell) splits naturally prior to maturity. This allows pistachios to be marketed largely in-shell for fresh consumption. Flavonoids and resveratrol have been mainly reported in pistachio nuts and the skin. Major phenolic constituent of pistachio hull has been reported as gallic acid: the amount was estimated to be 79 and $82 \%$ for the Damghan and Kerman varieties, respectively (Vahabzadeh et al., 2004). Vahabzadeh et al. (2004) extracted the phenolic compounds from two different varieties (Kerman and Damghan) of pistachio hulls using different aqueous solutions of methanol and ethanol and found that aqueous ethanol solution gave the highest yield of phenolic extract. Further, it exhibited strong antioxidant activity, comparable with that of the synthetic antioxidant BHA (i.e., 98 vs. 99\%). A Similar observation has been made on the stability of soybean oil by Goli et al. (2005) who reported that pistachio hull extract was effective in retarding oil deterioration at $60{ }^{\circ} \mathrm{C}$, with activity increasing with concentration in the range $0.02-0.06 \%$ and a concentration of $0.06 \%$, pistachio hull extract was similar in activity to BHA and BHT added at $0.02 \%$. In addition, they found that the amount of phenolic compound in the water extracts (in either solvent or ultrasound-assisted solvent extraction method) was highest when compared to other extraction methods. Arcan and Yemenicioğlu (2009) found that removal of seed coat of pistachio nut caused 55\% reduction in the total antioxidant activity. Farahpour et al. (2015) studied the effect of Pistacia atlantica hull extract on antioxidant and biological activities of pistachios. They reported that the hull extract showed significantly higher antioxidant activity compared to ascorbic acid. Moreover, the hull extract reduced wound healing time which was then suggested to be used in wound healing ointment. Garavand et al. (2015) used ultrasound and microwave to evaluate their effect on the extraction of phenolic compounds from pistachio hulls. They found that ultrasound-assisted extraction increased phenolic compounds (vanillic acid, $p$-coumaric acid, naringenin, and catechin) as determined by using HPLC-MS. In addition, microwave-assisted extraction increased phenolic contents in a power-dependent manner.

\subsection{Walnut}

The walnut tree (Juglans regia L.) is cultivated commercially throughout southern Europe, northern Africa, eastern Asia, the 
<smiles>[R]c1cccc(O)c1C(=O)O</smiles>

\section{Anacardic Acids}

1. $\mathrm{R}=\mathrm{C}_{15: 0}$, 6-pentadecyl salicylic acid

2. $\mathrm{R}=\mathrm{C}_{15: 1}, 6-[8(\mathrm{Z})$-pentadecenyl $]$ salicylic acid

3. $\mathrm{R}=\mathrm{C}_{15: 2,}, 6-[8(\mathrm{Z}), 11(\mathrm{Z})$-pentadecadienyl $]$ salicylic acid

4. $\mathrm{R}=\mathrm{C}_{15: 3,6}$-[8(Z), 11(Z), 14 pentadecatrienyl] salicylic acid<smiles>[R]c1cc(O)cc(O)c1</smiles>

\section{Cardols}

1. $\mathrm{R}=\mathrm{C}_{15: 0,}$ 5-pentadecyl recorcinol

2. $\mathrm{R}=\mathrm{C}_{15: 1}, 5-[8(Z)$-pentadecenyl $]$ recorcinol

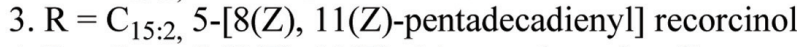

4. $\mathrm{R}=\mathrm{C}_{15: 3}, 5-[8(\mathrm{Z}), 11(\mathrm{Z}), 14$ pentadecatrienyl $]$ recorcinol

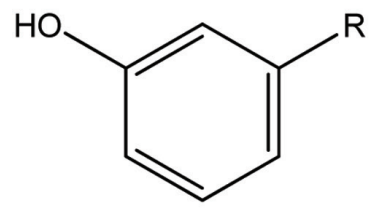

\section{Cardanols}

1. $\mathrm{R}=\mathrm{C}_{15: 0}$, 3-pentadecyl phenol

2. $\mathrm{R}=\mathrm{C}_{15: 1,3}, 3-[8(\mathrm{Z})$-pentadecenyl $]$ phenol

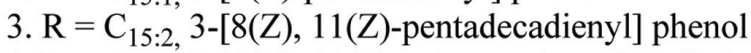

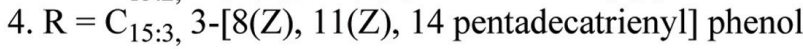

Figure 30. Chemical structures of some of the phenolic compounds contained in CNSL. Source: Adapted from Kubo et al., (1993a).

USA and South America. An edible seed, the kernel forms 42-60\% of the weight of the mature nut, depending mainly on the variety (Labuckas et al., 2008). The slightly astringent flavour of walnut fruit has been associated with the presence of phenolic compounds (Colaric et al., 2005). In walnut, inside the fruit shell a special protective tan-brown skin known as the pellicle, which is only $5 \%$ of the fruit weight, surrounds the kernel. Although the pellicle contributes only $5 \%$ to the fruit weight, its total phenolic is at least 93-97\% higher than that of whole kernels (Labuckas et al., 2008). According to Arcan, and Yemenicioğlu (2009) the removal of the seed coat reduced the total phenolic content and antioxidant activity of fresh walnuts by almost $75 \%$ and $90 \%$ respectively. Fukuda (2009) measured the presence of seven major polyphenols (casuarinin, casuarictin, pedunculagin, rugosin, tellimagrandin 1, tellimagrandin 11, ellagic acid) in American, Chinese, and Japanese variety of five commercial walnuts by HPLC and interestingly noted that no polyphenols were detected in walnuts from which the pellicle was removed.

Most phenolic compounds commonly identified in walnut are phenolic acids, hydrolysable tannins and condensed tannins (Colaric et al., 2005; Fukuda, 2009; Labuckas et al., 2008;) (Figure 31). Thirty-two polyphenolic compounds include hydrolysable tannin monomers (25) and dimmers (4), ellagic acid derivatives (2) and catechin have been reported in walnut (Fukuda, 2009). Fukuda et al. (2004) analyzed the quantity of polyphenols, mainly hydrolysable tannins (ellagitannins) in walnut polyphenol rich extract and found that main polyphenol was pedunculagin $(16 \%$ per walnut polyphenol fraction weight). Colaric et al. (2005) analyzed the ripe fruits of 10 walnut cultivars and identified phenolic acids (chlorogenic, caffeic, $p$-coumaric, ferulic, sinapic, ellagic and syringic acid) and syringaldehyde and juglone. They found that syringic acid was the most abundant phenolic of the ana- lyzed walnut kernel and seed coat (average of all cultivars was $33.83 \mathrm{mg} / 100 \mathrm{~g}$ in kernel and $1003.24 \mathrm{mg} / 100 \mathrm{~g}$ in seed coat) followed by juglone (11.75 and $317.90 \mathrm{mg} / 100 \mathrm{~g}$ in kernel and seed coat, respectively). In seed coat as well as in kernel the lowest contents of ferulic acid and sinapic acid were found among the identified phenolics. According to the study of Colaric et al. (2005), the concentrations of abundant phenolic compounds, juglone, syringic and ellagic acid, in walnut seed coat (pellicle) are over 20 -fold higher than the concentrations of these compounds in the seed. Slatnar et al. (2015) identified catechin, ferulic acid, caffeic acid, and neochlorogenic acid as well as 13 gallic acid oligomers, 4 procyanidin B2 isomers, 18 ellagic acid derivatives, and 3 quercetin glycosides present in common walnuts. More recently, 16 phenolics within black walnut kernels including gallic acid, catechin, 1,3,6-trigalloylglucose, hydroxybenzoic acid, vanillic acid, syringic acid, caffeic acid, rutin, quercetin glucoside, epicatechin gallate, ellagic acid, naringin, coumaric aicd, ferulic acid, and cinnamic acid were accurately quantified through using LC-QqQ-MS/MS (Vu et al., 2018).

Labuckas et al. (2008) examined the phenolic content and antioxidant activity of the kernel and seed coat (hull) of three walnut varieties with two different solvent systems and showed the superiority of seed coat extract. In another study, Samaranayake et al. (2008) investigated the phenolic contents and their antioxidative capacities (using different in vitro model systems) of different fractions (contents of whole nut, skin and kernel) of English walnut (Juglans regia L.) and reported that phenolic compounds extracted ( $95 \%$ ethanol) from walnut skin contained the highest amount of total phenolics and exhibited the highest antioxidative capacity as evaluated by the trolox equivalents antioxidant capacity assay.

Phenolic compounds from walnut fruits have a positive influence on human health such as a decrease of coronary heart dis- 


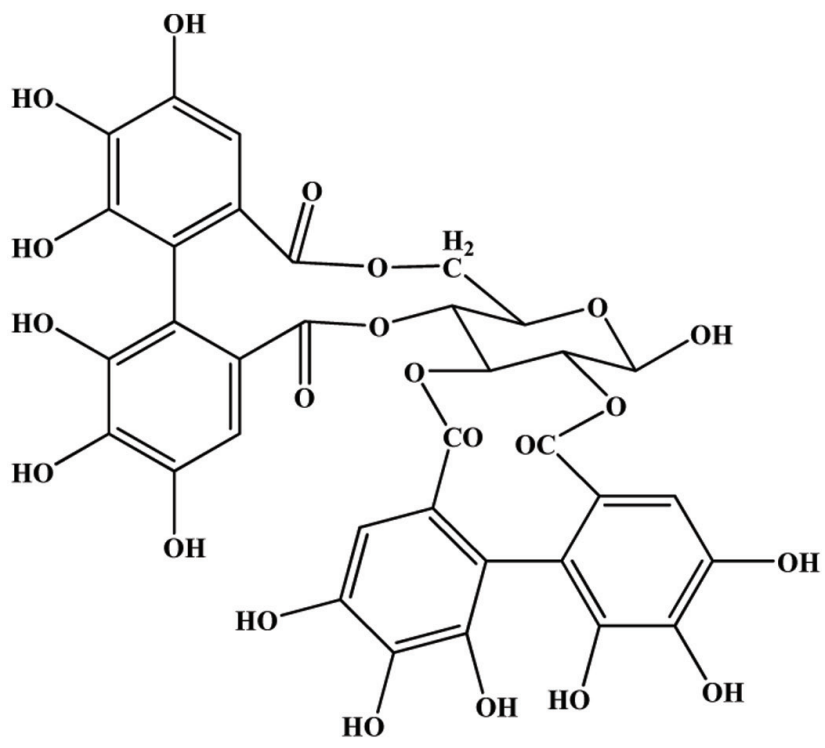

Penducalagin<smiles>O=c1oc2c(O)c(O)cc3c(=O)oc4c(O)c(O)cc1c4c23</smiles>

Ellagic acid

Figure 31. Structure of major polyphenols identified in walnut. Source: Adapted from Alasalvar and Shahidi, (2009).

eases, prevention of several kinds of cancer, and anti-inflammatory and antimutagenic activities (Anderson et al., 2001; Fukuda et al., 2003; Gunduc and El, 2003; Horton et al., 1999; Lavedrine et al., 1999; Zambon et al., 2000). In spite of these beneficial effects, walnut phenolics may adversely influence the protein solubility (Sze-Tao and Sathe, 2000).

\section{By-products of legumes}

\subsection{Peanut}

Peanuts (Arachis hypogaea L.) belong to the leguminosae family and often referred to as groundnuts. The edible parts of peanuts consist of the kernel and protective skin. The kernels are used to make peanut butter, roasted snack peanuts, peanut confections, and peanut oil while the peanut skin, which has a pink-red colour and astringent taste, is typically removed before peanut consumption or inclusion in confectionary and snack products (Yu et al., 2005). Peanut skin is rich in phenolics and potentially other health promoting compounds (Huang et al., 2003; Lou et al., 1999; Nepote et al., 2002; Yen et al., 2005; Yu et al., 2005).

Biologically active components and nutraceuticals in peanuts and related products have been the subject of a review by Isanga and Zhang (2007). Peanut skin contain three classes of phenolics, including phenolic acids (caffeic acid, cinnamic acid, protocatechuic acid, resorcylic acid, salicylic acid, chlorogenic acid, ferulic acid and coumaric acid), flavonoids (catechins and procyanidins), equol, morelloflavone, sciatopitysin, and methylcatechin), naphthoquinione (plmnbagin), coumestrol (psoralidin), coumarin (daplmoretin, dicoumoxyl), and stilbene (resveratrol) (Yu et al., 2005; Toomer, 2018). Earlier, Lou et al. (1999) reported the presence of 6 A-type procyanidins in peanut skin. Yu et al. (2005) found that 1 g dry peanut skin contains $90-125 \mathrm{mg}$ total phenolics depending on the effects of skin removal methods (direct peeling, blanching, and roasting). In another study, total phenolics in methanolic and ethanolic extracts from defatted peanut skins were 158.6 and 144.1 $\mathrm{mg} / \mathrm{g}$, respectively (Nepote et al., 2002).

The major flavonoid compound of peanut hull is luteolin (Daigle et al., 1988; Duh et al., 1992; Yen and Duh, 1995) (Figure 32). Duh et al. (1992) reported that the total phenolic content of peanut hulls were $41.8,19.9$ and $7.3 \mathrm{mg} / \mathrm{g}$ peanut hulls in methanol, ethanol and acetone extraction respectively. Yen and Duh (1995) examined the concentration of phenolic compounds in the peanut hulls of four different cultivars using methanol as extraction solvent and found that the luteolin content ranged from 0.95 to $3.16 \mathrm{mg} / \mathrm{g}$ of hulls and the total phenolic content was 4.2 to $10.2 \mathrm{mg} / \mathrm{g}$ of hulls. These results indicate that peanut skins could be a higher source of phenolic compounds and natural antioxidant components than peanut hulls. The study of Daigle et al. (1988) and Yen et al. (1993) showed that the total phenolic content increased with maturity of peanut hulls.

The compounds found in peanut skin are considered potent antioxidants, particularly, flavonoids resveratrol, and tannins (Yu et al., 2005; Oldoni et al., 2016). In peanut hulls, the presence of luteolin (Duh et al., 1992) and high total phenolic content were associated with a higher the antioxidant activity (Yen et al., 1993). The comparative study of total antioxidant activities of peanut skin extracts and green tea infusions demonstrated that peanut skin extracts had chemically higher antioxidant potential than green tea infusions (Yu et al., 2005). Huang et al. (2003) isolated and identified ethyl protocatechuate (EP), from peanut skin and showed that EP contributed to the antioxidant activity. Keefe and Wang (2006) reported that the phenolic compounds extracted from peanut skins could significantly reduce the oxidation of meat products and extend their storage stability. In an interesting study, de Camargo et al. (2014b) added peanut skin to cookies and evaluated their composition, antioxidant capacities and sensory quality. They found that the insoluble fiber content and the total phenolic contents were increased by up to 52 and $30 \%$, respectively. All antioxidant activity tests showed significant increases by increasing the addition level of the skin. More importantly, the cookies prepared were well accepted by sixty volunteers, thus suggesting that peanut skin might potentially be used as a cookie ingredient which could also improve the health status of consumers. 
<smiles>O=c1cc(-c2ccc(O)c(O)c2)oc2cc(O)cc(O)c12</smiles>

Figure 32. Structure of major flavonoid in peanut hull.

\subsection{Beans}

Beans are a good source of starch, dietary fiber, protein, and minerals and they also serve as a rich source of bioactive constituents (Geil and Anderson, 1994). Beans have gained increasing attention as a functional food item because like other legume seeds, the common bean seed contains a number of bioactive compounds such as enzyme inhibitors, lectins, phytates, oligosaccharides, and phenolic substances that may play metabolic roles in humans or animals that frequently consume this food (Díaz-Batalla et al., 2006). In legume seeds, phenolics are mainly located in the seed coats, but also in cotyledons in bound forms as part of cell walls (Duenas et al., 2006; Madhujith and Shahidi 2005b). Mostly in processing/utilization, dehulling is a pre-requisite for bean, hence the seed coat is a by-product.

Seed coat phenolics like condensed tannins, anthocyanins, and other flavonoids such as quercetin and kaempferol glycosides are reported in bean seed coats (Aparicio-Fernández et al., 2005; DíazBatalla et al., 2006; Espinosa-Alonso et al., 2006; Ranilla et al., 2007; Romani et al., 2004). Beninger and Hosfield (1998) reported that the pigments responsible for seed coat colour in beans are flavonoids. Moreover, the wide variety of seed coat patterns and colour in common beans ( $P$. vulgaris L.) is controlled by a group of well-defined genes that appear to regulate the flavonol and anthocyanin biosynthetic pathways (McClean et al., 2002). Espinosa-Alonso et al. (2006) showed that total phenol and condensed tannin contents in P. vulgaris tended to increase according to clearness of seeds, and conversely, Barampama and Simard (1993) reported that beans with light coloured coats have lower tannin contents than beans with dark pigmented coats such as black beans.

The study of Madhujith and Shahidi (2005b) and Madhujith et al. (2004) showed that beans with coloured seed coat rendered strong antioxidative activity and antiradical capacity. They found that among red, brown, and black beans, red and brown beans exhibited the highest potential in scavenging free radicals. In this study, Madhujith and Shahidi (2005b) analysed the four bean varieties (Phaseolus vulgaris L.) (white kidney, red pinto, swedish brown, and black kidney) and their hull fractions ( $80 \%$ acetone) and found that total phenolic content of bean hulls and whole seed extracts ranged from 6.7 to 270 and 4.9 to $93.6 \mathrm{mg} / \mathrm{g}$ extract as catechin equivalents, respectively.

In another study, Ranilla et al. (2007) analysed the phenolic profile and antioxidant capacity of Brazilian and Peruvian bean cultivars and found that the seed coat colour pattern and the type of cultivar showed an important influence on the variability of phenolic profiles and levels, respectively. Their work exhibited that certain groups of seed coat colours such as brown and red groups tended to have higher levels of total phenolics and condensed tannins than the black group, and in contrast, less coloured coats such as white and yellow types did not exhibit considerable contents of these compounds. Further, they reported that phenolic compounds like condensed tannins were strongly correlated with the antioxidant capacity in seed coats. In this study, the total phenolic contents varied from 0.46 to $86 \mathrm{mg}$ catechin equivalents $/ \mathrm{g}$ seed coat and condensed tannins ranged from 11.5 to $449 \mathrm{mg}$ (catechin equivalents)/g seed coat. In the same study, they reported the presence of high quantity of total anthocyanins, ranging from 265 to $558 \mathrm{mg} / 100 \mathrm{~g}$ (expressed as cyanidin) in black bean seed coat. Salinas-Moreno et al. (2005) found a range from 1,010 to 1,810 $\mathrm{mg} / 100 \mathrm{~g}$ total anthocyanins in black bean seed coat of Mexican cultivars and Takeoka et al. (1997) reported that the content of $2,370 \mathrm{mg} / 100 \mathrm{~g}$ of seed coat from a cultivar developed in an experimental station in the U.S.A. Astadi et al. (2009) identified the individual anthocyanidins such as delphinidin, cyanidin, and pelargonidin by HPLC coupled with diode array detector in black soybean coat and reported that the content of phenolic and anthocyanins ranged from 6.46 to $8.15 \mathrm{~g}$ (gallic acid equivalents) $/ 100 \mathrm{~g}$ fresh weight and 1.36 to $1.45 \mathrm{~g} / 100 \mathrm{~g}$ fresh weight, respectively. Similarly, the black soybean, especially its seed coat with abundant anthocyanins was reported to have the highest antioxidant activity among different soybeans, i.e. green, yellow, and red-brown soybean, owing to its higher total phenolic content in both free and bound form (Furuta et al., 2003; Peng et al., 2018). Meanwhile, around 60 free phenolics and 53 bound phenolics were identified along with the accurate quantification of 28 free/bound phenolics in seed coat and cotyledons of black soybean by using LC-MS/MS (Peng et al., 2018).

In addition to these works, many authors have observed high antioxidant activity in the seed coat of black gram, lentils, faba beans and peas, due to the essential fact that large amounts of phenolic compounds are located in this part of the seed (Amarowicz et al., 2000c; Cardador-Martínez et al., 2002; Girish et al., 2012; Nilsson et al., 2004; Shahidi et al., 2001; Takahata et al., 2001; Troszynska and Ciska, 2002; Zhang et al., 2018). An attempt was made to utilize chickpea and soybean husks as baking additives; increased antioxidant activity was found in DPPH radical and ABTS radical cation scavenging assays (Niño-Medina et al., 2019). Thus, the seed coat of legumes could be used as a valuable source of nutraceuticals.

\section{By-products of cereals}

Phenolic acids commonly found in many cereal grains and higher concentrations of these compounds are found in the outer layers of the kernel (Kim et al., 2006; Liyana-Pathirana and Shahidi, 2007). Hydroxycinnamate esters, particularly ferulate play an important role in strengthening the cell wall structure by cross-linking cell wall polysaccharides (Ishii, 1997; Ralph et al., 2004).

\subsection{Corn}

Corn is the most important cereal produced in the world (Zea mays L.) after wheat and rice. Many phenolics had been determined in different parts of maize plant, however, their concentration was found to be higher in corn flour (White and Xing, 1997). There are mainly two groups of phenolic acids in cereal bran: benzoic and cinnamic acid derivatives. Ferulic acid and other hydroxycinnamic acids (caffeic and $p$-coumaric acid derivatives) have been found to 
have good antioxidant activities (Kim et al., 2006). Hydroxycinnamic acids (ferulic and $p$-coumeric) and vanillin have been reported in corn bran (Barberousse et al., 2008: Bonnin et al., 2002: Buranov and Mazza 2009). Ferulic acid content is more significant in wheat and corn bran amount of 5 and $50 \mathrm{~g} / \mathrm{kg}$, respectively, which is also the precursor of vanillin (Ou and Kwok, 2004). In another study, Bunzel et al. (2005) identified four new phenolic dimers and trimers that contain ferulic acid moieties in corn bran.

Corn tassel, a by-product of corn is the source of phenolic compounds (Mohsen and Ammar, 2009). Mohsen and Ammar (2009) extracted the phenolic compounds using different solvent and reported that ethanol yielded highest extraction followed by methanol and water, where the total phenols were $0.1575,0.1125$ and $0.0737 \%$, respectively. Corncob is one of the most plentiful and important agriculture by-product accounts for up to $50 \%$ of the total corn seed production (Sultana et al., 2007). Sultana et al. (2007) reported that methanolic extract of corn cob had higher phenolic content and best antioxidant activity and the content of total phenolics extracted by different solvent ranged from 1.2 to $4.2 \mathrm{~g}$ (gallic acid equivalents) $/ 100 \mathrm{~g}$ dry matter. The amount of total phenolics $(4.2 \%)$ in corncob is greater than rice bran $(0.36 \%)$ (Iqbal et al., 2006), and wheat bran (0.10\%) (Zhou and Yu, 2004). In a recent study, Vazquez-Olivo et al., (2019) compared total phenolic contents (TPC) of grain, husk, leaf, cob, root, and stem; husk showed the highest TPC whereas grain exhibited the lowest TPC among all tested samples. However, according to antioxidant activity tests, cob and stem showed better antioxidant activity than other corn by-products.

\subsection{Rice}

Rice is the principal cereal in Asia, some countries in Africa, and Latin America and the staple food of nearly half of the world's population. During rice processing rice bran is produced as a by-product of rice milling. The rice bran comprises many health beneficial components such as sterols, higher alcohols, gammaoryzanol, tocopherols, tocotrienols and phenolic compounds (Aguilar-Garcia et al., 2007; Nicolosi et al., 1994).

Chotimarkorn et al. (2008) investigated the antioxidant components of rice bran from five varieties of long grain rice, cultivated in Thailand and found that total phenolic contents were ranged from 2.2 to $3.2 \mathrm{mg}$ (gallic acid equivalents) per gram rice bran and the total flavonoid were content ranged from 0.03 to $0.1 \mathrm{mg}$ (catechin equivalents) per gram rice bran. According to the study of Aguilar-Garcia et al. (2007), total phenolic content of rice bran was ranged between 6.04 and $6.84 \mu \mathrm{mol}$ gallic acid equivalents per gram.

Rice hull contain mainly phenolic acids such as phytic acid, vannillic acid, syringic acid and ferulic acid (Osawa et al., 1985; Ramarathnam et al., 1989; Wu et al., 1994; Asamarai et al., 1996). Ramarathnam et al. (1989) reported the presence of iosvitexin $(C$ glycosyl flavonoid) (Figure 33), which was found to be absent in the hulls of short-life rice seeds.

A study on wild rice hull antioxidants, Lee et al. (2003) identified $p$-coumaric acid, 3-vinyl-1-oxybenzene, $p$-hydroxybenzaldehyde, vanillin, $p$-hydroxybenzoic acid, and 4,7-dihydroxyvanillic acid by GC-Ms analysis in FIR-irradiated rice hull methanolic extract. In this study they found that after 30 min of FIR treatment total phenol contents of rice hull extracts increased from 0.12 to $0.19 \mathrm{mM}$. A study on Sultana et al. (2008) showed the total phenolic content of rice hull $(2 \mathrm{~g} / \mathrm{kg})$ was half the amount of rice bran. Wanyo et al. (2014) investigated the antioxidant activity of rice bran, rice husk, and ground rice husk treated by hot air, far-infra- red radiation, and cellulose. They found that far-infrared radiation treatment showed better antioxidant activity than other treatments. Grounding of rice husk could also improve their antioxidant activity.

\subsection{Wheat}

Wheat bran, an important by-product of the cereal industry, is rich in potentially health-promoting phenolic compounds (Bauer et al., 2012; Wang et al., 2008; Laddomada et al., 2015). The majority of phenolic acid in wheat bran are esterified and bound to cell wall components (Beta et al., 2005; Liyana-Pathirana and Shahidi, 2006; Laddomada et al., 2015). In wheat bran, ferulic acid is predominate phenolic acid accounted for more than $40 \%$ of the total phenolic acids on a per weight basis, along with significant levels of syringic, $p$-hydroxybenzoic, vanillic, and coumaric acids (Graf, 1992, Kim et al., 2006; Onyeneho and Hettiarachchy, 1992; Zhou et al., 2004a, 2004b, 2005; Zhou and Yu, 2004; Pasqualone et al., 2015). In addition, Kim et al. (2006) detected caffeic, salicylic and trans-cinnamic acids. The majority of the phenolic acids in a wheat bran existed in a bound form can be released by hydrolyzing the bran under alkaline or acidic conditions (Kim et al., 2006).

Liyana-Pathirana and Shahidi (2007) reported the presence of higher phenolic content in wheat bran compare to other fractions and found that total phenolic content of wheat bran was 2.3-3.4 $\mathrm{mg}$ ferulic acid equivalents/g defatted material. Similar observation has been reported in ultrasound-assisted extraction by Wang et al. (2008) who found that under the optimum condition $(64 \%$ ethanol; extraction temperature, $60{ }^{\circ} \mathrm{C}$; and extraction time, 25 $\mathrm{min}$ ) total phenolic content of wheat bran was $3.12 \mathrm{mg}$ (gallic acid equivalents $) / \mathrm{g}$.

Verma et al. (2008) measured the free, bound, and total phenolic content in the bran of 51 wheat cultivars. They found that free phenolic (extracted with $80 \%(\mathrm{v} / \mathrm{v})$ aqueous ethanol) content ranged from $854.1 \pm 265.1$ to $1,754.9 \pm 240.3 \mu \mathrm{g} / \mathrm{g}$ of bran gallic acid equivalents (GAE) and bound phenols ranged from 2,304.9 \pm 483.0 to $5,386.1 \pm 927.5 \mu \mathrm{g} / \mathrm{g}$ of bran (GAE), contributing $66-82 \%$ of the total wheat bran phenolic content. According to their study, total phenolic content ranged from $3,406.4 \pm 32.3$ to $6,702.7 \pm 19.6$ $\mu \mathrm{g} / \mathrm{g}$ of bran (GAE), with the average being $5,197.2 \pm 804.9 \mu \mathrm{g} / \mathrm{g}$ of bran (GAE). Pasqualone et al. (2015) investigated the effect of addition of aqueous extract of wheat bran, which was prepared by ultrasound-assisted extraction, to pasta on the antioxidant activity of the product. They found the extract contained ferulic acid and $p$-coumaric acid and the resultant pasta benefited from increased antioxidant activity.

\subsection{Buckwheat}

Watanabe et al. (1997) identified the presence of flavonoids such as quercetin, rutin, hyperin, vitexin and isovitexin and other phenolic compounds namely, protocatechuic acid, 3,4-dihydroxybenzaldehyde and proanthocyanidins in buck wheat hull. Among these, protocatechuic acid is the predominant $(13.4 \mathrm{mg} / 100 \mathrm{~g}$ of dried hulls) phenolic compound (Watanabe et al., 1997). Figure 34 shows the structures of isolated compounds from buckwheat hull. Another study (Hęś et al., 2012) extracted phenolic compounds using acetone, water, and methanol and compared unhulled buckwheat grain (UBG), hulled buckwheat grain (HBG), buckwheat hull $(\mathrm{BH})$, final bran (FB) and bran after grinding (GB). Methanol extract showed the highest total phenolic content. Among samples, buchwheat hull showed the highest total phenolic content when 


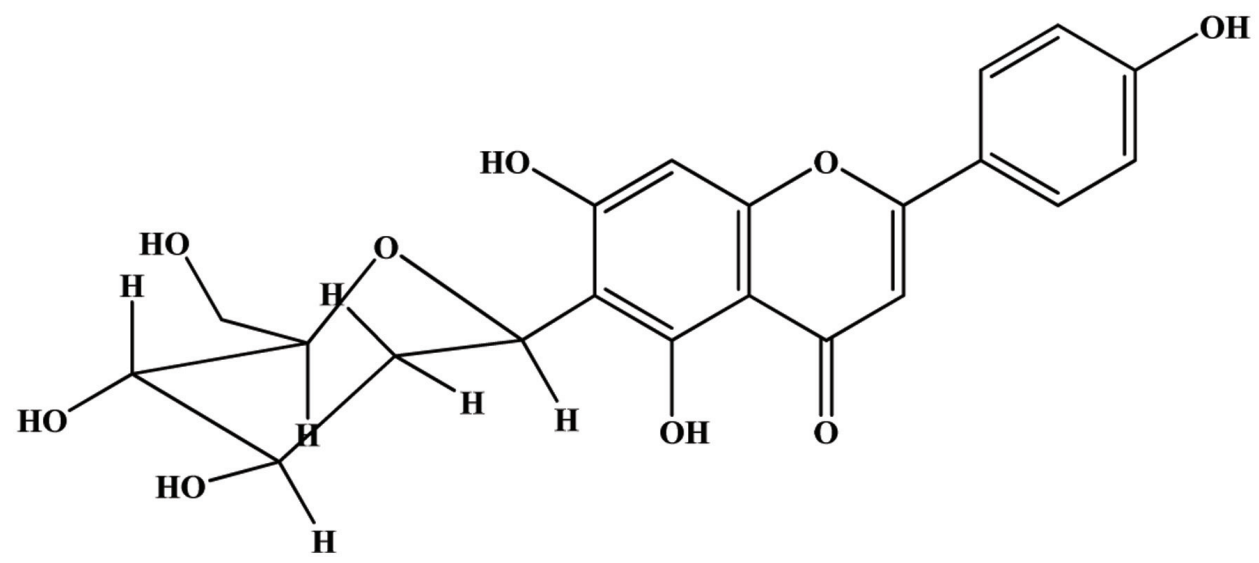

Figure 33. Isovitexin (C-glycosyl flavonoid) in rice hull. Source: Adapted from Ramarathnam et al., (1989).

extracted by both acetone and methanol, whereas water extracted hulled buckwheat grain had the highest total phenolic content. In addition, phenolic compounds from buchwheat hull are known to have antioxidant and cytoprotective capacities and buchwheat bran showed antioxidant and antiproliferative activities (Kabir et al., 2015; Li et al., 2016).

\subsection{Oat}

Oats are a main source of calorie and protein and their production is extensive and high (Hung, 2016). The total phenolic content of oat is $6.53 \pm 0.19 \mu \mathrm{mol}$ of gallic acid equivalent/g of gran (Hung, 2016). On the other hand, phenolic and hydroxyphenolic antioxidant compounds with acids, alcohols sugars or glycerides attached have been reported in oat hulls (Duve and White, 1991). In another study, Xing and White (1997) identified ten monophenolics such as ferulic, $p$-coumaric, $p$-hydroxybenzoic, vanillic, $o$-coumaric, sinapic, 4-hydroxyphenylacetic and salicylic acids, and vanillin and catechol in oat hull (Figure 35). They found that the ferulic acid was the predominant and the total phenolics were $560 \mathrm{mg} /$ $\mathrm{kg}$ oat hull. Another study (Zdunäczyk et al., 2006) reported that total phenolic compounds of husked oat, naked oat, and oat bran with hulls were $1.38 \pm 0.06,1.17 \pm 0.03$, and $1.55 \pm 0.03 \mathrm{mg} / \mathrm{g}$, respectively.

\section{By-products of oil seeds}

\subsection{Canola/rapeseed}

Canola and rapeseed contain 14 to $18 \%$ hulls. The hulls may contain up to $20 \%$ oil, $19.1 \%$ crude proteins $(\mathrm{N} \times 6.25), 4.4 \%$ minerals, and $48 \%$ dietary fibre and other constituents include simple sugars and oligosaccharides, polyphenolics, phytates, and residual polar lipids (Naczk et al., 2005). Phenolic acid such as sinapic acid and their derivatives, as well as soluble and insoluble tannins, are the predominant phenolic compounds found in canola and rapeseed (Amarowicz et al., 2000b).

Canola hulls have been reported to contain $6 \%$ of phenolic compounds (Naczk and Shahidi, 1998). These compounds mainly include phenolic acids (Krygier et al., 1982; Amarowicz et al., 2000a) and soluble and insoluble condensed tannins (Amarowicz et al., 2000b; Naczk et al., 1994, 2000; Naczk and Shahidi, 1998).
The presence of condensed tannins in rapeseed hulls was first reported by Bate-Smith and Ribereau-Gayon (1959). This finding was verified by Durkee (1971), who identified cyanidin, pelargonidin and later Leung et al. (1979) reported that condensed tannins of rapeseed hulls contained leucocyanidin as their basic units.

Mitaru et al. (1982) found that rapeseed hulls contain 0.02 $0.22 \%$ condensed tannins. Rapeseed hulls contain no more than $0.1 \%$ condensed tannin extractable by solvent systems commonly used for isolation of polyphenols (Leung et al. (1979). The content of tannins in rapeseed/canola hulls ranged from 0.014 to 2.3 $\mathrm{g}$ tannin per $100 \mathrm{~g}$ hulls (Amarowicz et al., 2000b; Naczk et al., 1994; Shahidi and Naczk, 1995). Tannin contents within a particular canola cultivar varied by a factor of 9 to 15 and tannin extracts from canola hulls contained approximately $20 \%$ proanthocyanidins (Naczk et al., 1994; and Shahidi and Naczk, 1995). Study of Amarowicz et al. (2000c) and Naczk et al. (2005) showed that total phenolic content of canola hull ranged from 94.3 to $296 \mathrm{mg} / \mathrm{g}$ (sinapic acid equivalents). Both cultivar differences and environmental growing conditions may influence the content of condensed tannins and total phenolics in canola hulls.

Amarowicz et al., (2000a) investigated the antioxidative activities of non-tannin fractions of phenolic compounds in acetone $(70 \%, \mathrm{v} / \mathrm{v})$ extract isolated from canola hulls. They isolated five major fractions according to UV absorption and on the basis of thin layer chromatograms, only the presence of free ferulic acid and sinapine were confirmed in the non-tannin fractions of phenolic compounds. However, all fractions of canola hull non-tannin phenolics exhibited strong antioxidant activities in a $\beta$-carotenelinoleate model system. Further work is required to isolate and identify the active components of canola hull phenolics. Interestingly, Terpinc et al. (2012) observed that a negative correlation between total phenolic content and the antioxidant efficiencies of the extracts obtained from oil cakes and they proposed that phenolic compounds are not the only contributors to the antioxidant activities of the oil cakes. Teh et al. (2015) reported that both microwave and pulsed electric field assisted extraction could be used for phenolic compound extraction from canola seed cake.

\subsection{Olive}

The by-products of the olive industry such as olive mill waste water (OMWW) and olive leaves have attracted considerable interest as a source of phenolic compounds (Balasundram et al., 2006). The olive leaf is a well-known source of biophenols that is marketed 
<smiles>[R10]OC(=O)OCCCCCO</smiles><smiles>[R]c1c(O)c([R2])c2oc(-c3ccc(O)cc3)cc(=O)c2c1O</smiles>

$\mathrm{R}$

$\mathrm{H}$

Galactose

Rutinose

$\begin{array}{lll} & \text { R1 } & \text { R2 } \\ \text { Vitexin } & H & \text { Glucose } \\ \text { Isovitexin } & \text { Glucose } & H\end{array}$

Figure 34. Structure of flavonol, C-glycosyl flavone isolated from buckwheat hulls. Source: Adapted from Watanabe et al., (1997).

under multiple trade names as a nutraceutical. Benavente-Garcia et al. (2000) found that oleuropein was the major phenolic compound in olive leaves, followed by hydroxytyrosol, luteolin-7-glucoside, apigenin-7-glucoside, and verbascoside.

Phenyl acids, phenyl alcohols (3,4-dihydroxyphenyl ethanol and $p$-hydroxyphenyl ethanol), flavonoids (flavonol glycosides, luteolin-7-glucoside, and rutin, and the anthocyanins, cyanidin, and delphinidin glycosides), and secoiridoids (oleuropein, demethyloleuropein) (Figure 36) are the most important classes of phenolic compounds reported in olive fruit (Macheix et al., 1990). The identified phenolic compounds in OMWW and their concentrations vary from one study to another. According to Feki et al. (2006), this could be related to the olive variety, climatic conditions, the period of harvest, and/or to the olive oil extraction system. Visioli and Galli (2003) reported that phenolic content of the OMWW fluctuated between 1.0 and $1.8 \%$ depending on varietal factors and processing effects.

Hydroxytyrosol, tyrosol, oleuropein, and a variety of hydroxycinnamic acids have been reported as the major phenolic constituents in OMWW (Feki et al., 2006; Obied et al., 2005). Casa et al. (2003) identified catechol, 4-hydroxybenzoic acid, 4-methylcat- echol, 3-hydroxyphenylpropionic acid, 3,4,5-trimethoxybenzoic acid and trans-cinnamic acid, as major compounds. Bianco et al. (2003) identified 20 phenolic compounds in OMWW using HPLCMS/MS and they were able to quantify 16 of these compounds. These phenolic compounds may be either naturally occurring in the fruits or formed during processing, where they partition between oil and waste phases.

Servili et al. (1999) compared the phenolic profiles of olive fruit (cv. Frantoio) and the corresponding oil, pomace, and OMWW. The HPLC profiles of the fruit showed similarity to that of the pomace where secoiridoid glycosides such as oleuropein and demethyloleuropein were present in high concentration. In contrast, secoiridoid derivatives, namely, hydroxytyrosol and oleuropeindial, were dominant in OMWW. Feki et al. (2006) studied the changes in polyphenol fraction, particularly hydroxytyrosol of OMWW in fresh and stored samples and found that significant accumulation of hydroxytyrosol in stored OMWW and the concentrations of the other phenolic compounds were markedly decreased. This evolution can be attributed to some hydrolysis reactions of oligomeric polyphenols (hydroxytyrosol derivatives), which are composed of hydroxytyrosol units, attached to<smiles>O=C(O)Cc1ccc(O)cc1</smiles>

4-Hydroxyphenylacetic acid<smiles>O=C(O)c1ccccc1O</smiles>

Salicylic acid<smiles>COc1cc(C=O)ccc1O</smiles>

Vanillin<smiles>Oc1ccccc1O</smiles>

Catechol

Figure 35. Some monophenolic compounds identified in oat hull. 


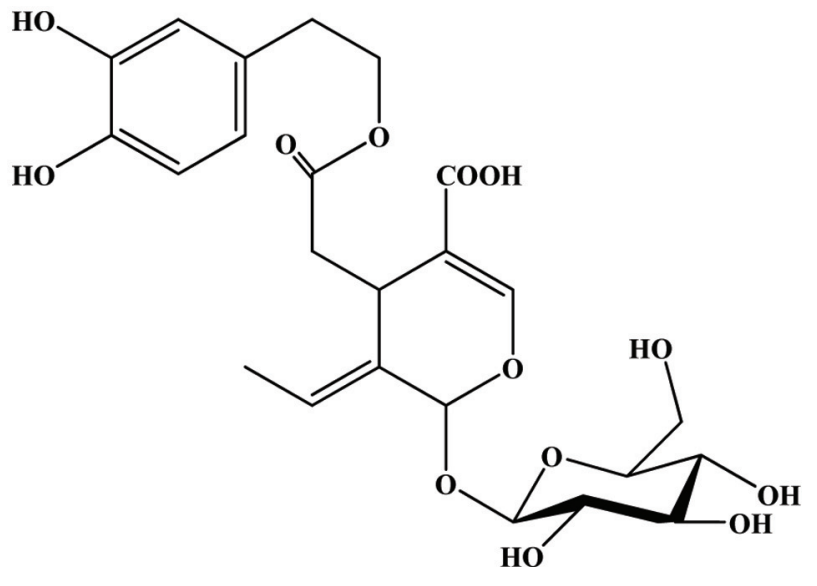

Figure 36. Structure of demethyloleuropein.

other compounds via ester or/and glucosidic linkages (Feki et al., 2006). Yakhlef et al. (2018) compared the phenolic profile of virgin olive oil, pomace, and OMWW of three different cultivars (Blanquette, Rouguette and Sigoise). They found OMWW of all caltivas showing the highest total phenolic contents and this exhibited very strong antimicrobial activity. However, the olive oil comprises only $2 \%$ of the total phenolic content of the olive fruits, with the remaining $98 \%$ being lost in olive mill waste (OMW) (Rodis et al., 2002). Therefore, olive mill wastes are the major potential source of phenolic compounds.

\subsection{Sunflower oil}

While the vibrant, strong sunflower (Helianthus annuus L.) is a recognized worldwide for its beauty, it is also an important source of food. Sunflower oil is a valued and healthy vegetable oil and sunflower seeds are enjoyed as a healthy, tasty snack and nutritious ingredient to many foods. Based on production (13.0 and15.9 million metric tons in 2009 and 2014, $10 \%$ of the total global vegetable oil consumption in 2009) sunflower oil is ranked fourth next to palm, soy and rapeseed oil (FAO-STAT-2010 and 2014). Sunflower seeds, both confectionery and oilseed may be sold as in-shell seeds or dehulled kernels. The seeds can also be sprouted and eaten in salads. The majority of the oilseed varieties of sunflower seed are crushed after the hull is removed. Confectionery sunflower seeds fall into three categories: in shell, kernel, and birdseed. Larger sunflower seeds (in shell) are roasted, salted, and packaged for human consumption and are classified as either large or jumbo. Dehulling is commonly performed by cracking the hull with one's teeth and spitting it out while keeping the kernel in the mouth. Medium-sized seeds (kernel) are de-hulled and packaged for human consumption as well. These are primarily used for the bakery industry. Smaller seeds, known as "striped bird food," are sold in birdseed markets. Shells and residue originating from an oil extraction process are the major by-products of sunflower industry. Chlorogenic acid (5-O-caffeoylquinic acid) is the predominant phenolic acid reported in sunflower seed, meal and shell (Aramendia et al., 2000; Dreher and Holm, 1983; Leonardis et al., 2005; Pedrosa et al., 2000; Weisz et al., 2009).

With regard to the phenolic composition, Leonardis et al. (2005) isolated seven different compounds from sunflower seed shell by HPLC analysis. Among those, six were phenolic acids (chlorogenicacid, $o$-cinnamic acid, protocatechuic acid, caffeic acid, ferulic acid, and syringic acid) and one was not identified. In addition, their study deduced that the effectiveness of the sunflower antioxidant product was essentially similar to that of the caffeic acid standard, but $15-20 \%$ lower than that of propyl gallate.

In another study, Weisz et al. (2009) extracted and characterized eleven phenolic compounds such as 3-O-caffeoylquinic acid, 5- $O$ caffeoylquinic acid (chlorogenic acid), 4- $O$-caffeoylquinic acid, caffeic acid, caffeoylquinic acid, 5-O-p-coumaroylquinic acid, 5- $O$-feruloylquinic acid ferulic acid, 3,4-di- $O$-caffeoylquinic acid, 3,5-di- $O$-caffeoylquinic acid, and 4,5-di- $O$-caffeoylquinic acid from European origin non-oil seed and oil seed sunflower kernel and shell. According to their study, total phenolic content of shells ranged from $40.8 \mathrm{mg} / 100 \mathrm{~g}$ to $86.0 \mathrm{mg} / 100 \mathrm{~g}$ dry matter and sunflower kernels were up to 100 times higher than those determined in the shells. Although quantitative amounts markedly differed, phenolic subclasses in both shell and kernel were similar. In addition, they found that total phenolic content of press residue originating from oil extraction was $3 \%(2,940 \mathrm{mg} / 100 \mathrm{~g}$ dry matter $)$ in weight basis. This indicates oil press residue is an rich source of phenolic compounds and has the potential to be utilized in industrial level as per the rate of current production of sunflower seed.

\subsection{Sesame}

Sesame (Sesamum indicum L.) is cultivated in several countries such as India, Sudan, China and Burma, which are considered as the major producers $(60 \%$ of its total world production) (AbouGharbia et al., 2000). The conventional process for preparing sesame oil involves cleaning, optional dehulling, roasting, grinding, cooking, and preparing (Fukuda and Namiki, 1988). After dehulling, the sesame coat is commonly disposed of or used as animal feed. However, Abou-Gharbia et al. (1997) reported that the sesame oil from seeds with coat was more stable than those extracted from dehulled seeds. This observation may indicate that antioxidative components may exist in sesame coat. Therefore, use of sesame coat, after dehulling, as a source of natural antioxidants, may provide a means for utilization. Sesame cake is another byproduct of the oil industry is currently used as cattle feed. Suja et al. (2005) analysed the lignans (Sesamin, sesamolin and sesamol) in sesame cake qualitatively and quantitatively and reported the potency of antioxidant.

Lignans such as sesamin, sesamolin have been reported in sesame coat (Chang et al., 2002) while sesamol has not been detected in the same study. Shahidi et al. (2006) have shown that sesame coats possess high polyphenolic content, especially black sesame coats compare to sesame seeds. In this study the total phenolic content in black sesame and white sesame coat were $146.6 \mathrm{mg}$ and $29.7 \mathrm{mg}$ catechin equivalents per gram crude extract respectively. In another study, the total phenolics of sesame coat, derived as a by-product in dehulling and roasting process shown 598 and 260 mg per 100 gram dry matter, respectively (Elleuch et al., 2007).

A recent study (Görgüç et al., 2019) compared phenolic contents from sesame bran by using enzyme and ultrasound. They found that Alcalase was more suitable than viscozyme L. In addition, they also reported that $43{ }^{\circ} \mathrm{C}, 98 \mathrm{~min}, \mathrm{pH} 9.8,1.248 \mathrm{AU} / 100$ $\mathrm{g}$ (enzyme concentration), and $836 \mathrm{~W}$ (ultrasound power) were the optimum condition.

\subsection{Soybean cake}

Soybean cake is an important by-product produced during soybean oil processing, and has been shown to be a rich source of isofla- 
vone (Kao et al., 2008). Isoflavones in soybean and soybean products, present in four chemical forms: aglycone (daidzein, genistein and glycitein) (Figure 37), glucoside (daidzin, genistin and glycitin), acetylglucoside (acetyldaidzin, acetylgenistin and acetylglycitin), and malonylglucoside (malonyldaidzin, malonylgenistin and malonylglycitin) (Kao et al., 2008) (Figure 38).

Yang et al. (2019) recently studied the effect of fermentation of soybean meal on antioxidant activity. They reported that chlorogenic acid, vanillic acid, syringic acid, and $p$-coumaric acid were identified after steaming and fermentation, whereas these were not found in untreated soybean meal. Moreover, the phenolic contents were significantly increased upon fermentation.

\subsection{Flax shive}

Flax, Linum usitatissimum, is abundant in North America and is grown mainly for the production of seed used as a source of linseed oil. The stalk, which can be processed into fibre and ligninrich residue, is referred to as flax shive (Sharma, 1989). Generally, lignin contains considerable amounts of phenolic acids, which play a major role in the linkage of polysaccharides with lignin by ester and ether bonds (Saulnier et al., 1995). Furthermore, flax shives are known to be rich in guaiacyl units (75\%) (Buranov and Mazza 2008; Day et al., 2005) that are the precursors of vanillin. Linseed meal has been reported to contain hydroxycinnamic acid derivatives, $p$-coumaric acid glucoside and ferulic acid glucoside (Johnsson et al., 2002) and the flavonoids herbacetin diglucoside and kaempferol diglucoside (Qiu et al., 1999).

Phenolic constituents of flax shives are mainly consist of $p$ coumaric acid and ferulic acid (Buranov and Mazza, 2009). In addition, $p$-hydroxybenzaldehyde, vanillic acid, syringic acid, vanillin and acetovanillone have been identified in flax shive (Kim and Mazza, 2006; Buranov and Mazza, 2009). Ferulic acid is crosslinked with lignin and polysaccharides via ester and ether bonds, forming lignin/phenolics-carbohydrate complexes, which could be released with, dilute $(0.1-4 \mathrm{M}) \mathrm{NaOH}$ solution at $50-70{ }^{\circ} \mathrm{C}$ (Fry, 1982; Sun et al., 2002; Barberousse et al., 2008) or enzymatically by feruloyl esterases (Bonnin et al., 2002; Mathew and Abraham, 2004). Figure 39 shows the cleavage of lignin/phenolics-carbohydrate complexes in flax shives with alkali.

A detailed analysis of Buranov and Mazza, (2009) on flax shive, corn bran, and wheat bran showed that flax shives contained more p-coumaric acid $(61 \mathrm{mg} / 100 \mathrm{~g})$ than ferulic acid $(25 \mathrm{mg} / 100 \mathrm{~g})$ and ferulic acid in flax shive $(25 \mathrm{mg} / 100 \mathrm{~g})$ was much lower than wheat $(391 \mathrm{mg} / 100 \mathrm{~g})$ and corn $(2,510 \mathrm{mg} / 100 \mathrm{~g})$ bran, but the vanillin content of flax shives $(48 \mathrm{mg} / 100 \mathrm{~g}$ ) was comparable to corn bran $(55 \mathrm{mg} / 100 \mathrm{~g})$ and higher than wheat bran $(11 \mathrm{mg} / 100$ g). However, their result showed that overall content of hydroxycynnamic acids (ferulic and $p$-coumaric acids) in flax shives was lower $(86 \mathrm{mg} / 100 \mathrm{~g})$ than in wheat $(402 \mathrm{mg} / 100 \mathrm{~g})$ and corn bran $(2,860 \mathrm{mg} / 100 \mathrm{~g})$.

\section{By-products of beverages}

\subsection{Cocoa}

The cocoa tree belongs to the genus Theobroma (meaning "the food of the Gods") in the family sterculiaceae. Cocoa powder, cocoa nib and cocoa shell, cocoa leaves, which are normally wasted during frequent pruning, are the by-products derived from cocoa industry. Azizah et al. (1999) suggested that flavonoid compounds might be responsible for the antioxidative activities in cocoa powder, cocoa nib and cocoa shell based on their study on antioxidant property of cocoa by products using different solvents.

The presence of catechin-polyphenols such as epicatechin (EC), epigallocatechin gallate (EGCG), epigallocatechin (EGC), gallic acid (GA), and epicatechin gallate (ECG) has been reported in cocoa leaves (Osman et al., 2004). The comparative study of cocoa shoots, cocoa young leaves and tea leaves conducted by Osman et al. (2004) revealed that the phenolic content is significantly higher in cocoa leaves $(28.4 \%)$, than in cocoa shoots $(19.0 \%)$ and tea leaves $(17.3 \%)$. Further, the concentrations of epicatechin in cocoa shoots $(59.3 \mathrm{mg} / \mathrm{g})$ and in cocoa leaves $(28.2 \mathrm{mg} / \mathrm{g})$ were significantly higher that those found in green tea $(6.5 \mathrm{mg} / \mathrm{g})$ and the performance of cocoa shoots was very similar to the antioxidant activity of BHA. Based on this observation they suggested that the higher concentration of total polyphenols in cocoa leaves $(28.4 \%)$ could be due to the presence of other phenolics since the fresh cocoa leaves are reddish brown in color, indicating the presence of cyanidins. HernándezHernández et al. (2019) tested thermal treatment $\left(50-200{ }^{\circ} \mathrm{C}\right)$ to extract more phenolic compounds from cocoa bean husk. They found that temperatures of $150{ }^{\circ} \mathrm{C}$ and higher significantly increased total phenolic contents recoverable from cocoa bean husk.

\subsection{Tea [Camellia sinensis]}

Both green and black teas are manufactured from young shoots, mainly the first 2-4 leaves and a bud. Old tea leaves (OTL), which are not used in tea manufacture, and black tea wastes (BTW) are considered as agricultural by products. Green tea leaves (GTL) (Camellia sinensis L.) contain 10-30\% (dry leaf weight) of polyphenols, including catechins, flavonols, flavanones, phenolic acids, glycosides and the aglycones of plant pigments (Pan et al., 2003). Tea polyphenols are natural antioxidants (Tanizawa et al., 1984) and considered to be responsible for the anticarcinogenic and antimutagenic properties of tea, as well as protective action against cardiovascular diseases (Shahidi and Wanasundara, 1992; Tijburg et al., 1997; Wiseman et al., 1997).

Zandi and Gordon (1999) showed that the extracts from old tea leaves (OTL) have potential as natural antioxidants. The extraction yield and antioxidant activity of old tea leaves and black tea waste (BTW) extracts were reported by using various extraction methods with compared to green tea leaves (Farhoosh et al., 2007). In this study they found that BTW extracts had antioxidant activities comparable with or even better than those of OTL extracts while extraction yield decrease with increasing age of tea leaves. Tea extracts are powerful antioxidants, mainly owing to the presence of $(+)$-catechin, $(-)$-epicatechin, (-)-epigallocatechin, (-)-epigallocatechin gallate (Figure 40) and (-)-epicatechin gallate (Salah et al., 1995). Catechins are known to be non-volatile taste compounds of green tea (Nwuha et al., 1999) and present at 8-15\% of dry leaf weight (Goto et al., 1996). These compounds are effective free radical-scavengers (Salah et al., 1995) and also effective by metal chelation (Shahidi and Wanasundara 1992). Green tea and black tea wastes display antimicrobial and antioxidant activities due to their high content of tannin and catechin (Faustino et al., 2019; Farhoosh et al., 2007).

\section{Other bioactive compounds in by-products}

\subsection{Carotenoids}

Carotenoids are red, yellow, and orange pigments that are widely 


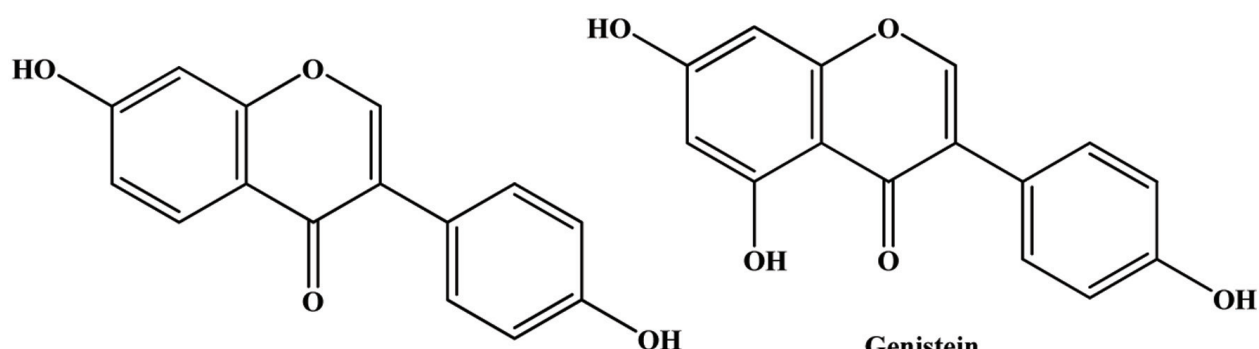

Daidzein

Genistein

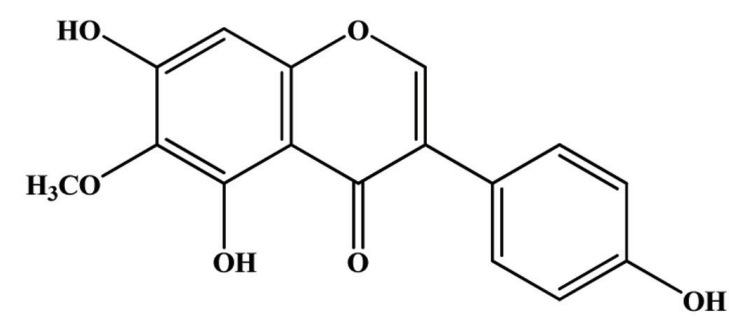

Glycitein

Figure 37. Structure of soy isoflavones identified in soybean cake.

distributed in nature. More than seven hundred naturally occurring carotenoids have been identified; of which as many as fifty may be absorbed and metabolized by the human body (Goodwin, 1994). However, only 14 carotenoids have been identified in human serum, with lycopene being the abundant (Krinsky, 1994). Carrots and tomatoes are the major dietary source of carotenoids.

Chemically, carotenoids can be divided into two major classes. The first class contains highly unsaturated hydrocarbon carotenoids such as lycopene, $\alpha$-carotene, $\beta$-carotene, $\gamma$-carotene, and $\zeta$ carotene, which have no oxygen and are usually orange and red in colour. Because they are highly unsaturated, they are particularly susceptible to oxidation. The second class contains the xanthophylls (lutein, zeaxanthin), which are oxygenated derivatives and have one or more oxygenated group substituents at particular sites on the terminal rings (Krinsky, 1994; Shi and Le Maguer, 2000; Young and Lowe, 2001).

Lycopene is an acyclic open-chain polyene with 13 double bonds and a molecular formula of $\mathrm{C}_{40} \mathrm{H}_{56}$. There are 11 conjugated double bonds arranged in a linear array, making it longer than any other carotenoid. Lycopene is more soluble in chloroform, benzene, and other organic solvents than in water. In aqueous systems, lycopene tends to aggregate and to precipitate as crystals. This behavior is suspected to lower lycopene bioavailability in humans (Zumbrunn et al. 1985). Lycopene is the red colored pigment abundantly found in red colored fruits and vegetables such as tomato, papaya, pink grapefruit, pink guava and watermelon (Choudhary et al., 2009; Kong et al., 2010). In ripe tomato fruits, lycopene exists as elongated, needlelike crystals. Lycopene is regarded as one of the most efficient singlet oxygen quencher and peroxyl radical scavenger of all the carotenoids and may represent an important defense mechanism in the human body. Singlet oxygen and free radicals can cause biological damage to important macromolecules and membrane constituents, and the presence of natural antioxidants may help to control these degradative reactions. Lycopene reacts synergistically with many other natural antioxidants to enhance their overall antioxidant activity (Kong et al., 2010). Lycopene is currently being added to products in the form of nutraceuticals, such as a concentrate in multivitamins or functional food products. Studies on the synergistic properties of lycopene are important and can be benefit for functional food and nutraceutical manufacturers and consumers.

\subsubsection{By-products of carrot}

Carrot (Daucus carota) is a good source of natural antioxidants, especially carotenoids (provitamin A and $\alpha$ - and $\beta$-carotenes) phenolic compounds (Chantaro et al., 2008; Zhang and Hamauzu, 2004). The $\beta$-carotene content in fresh carrot peels are similar to that reported in carrot flesh (Negi and Roy, 2001). Carrot byproduct (peel) possesses many bioactive compounds including $\beta$ carotene $(1.57 \mathrm{mg} / \mathrm{g} \mathrm{DW})$, lutein $(0.17 \mathrm{mg} / \mathrm{g} \mathrm{DW})$ and lycopene $(0.78 \mathrm{mg} / \mathrm{g} \mathrm{DW})$, which contribute to their potent antioxidant capacity (Lau et al., 2018).Juice yield is reported to be $60-70 \%$, and up to $80 \%$ of carotene may be lost with the pomace (Sims et al., 1993). According to Stoll et al. (2001), total carotene content of pomace may be up to $2 \mathrm{~g} / \mathrm{kg}$ dry matter, depending on processing conditions. Beta-carotene contents in carrot peels under different process conditions was examined by Chantaro et al. (2008) and they observed during blanching, a decrease in $\beta$-carotene content occurred as a result of thermal degradation and a significant loss of $\beta$-carotene was also observed after drying. Further, they noticed that the higher loss of $\beta$-carotene content was observed undergoing lower drying temperatures. Therefore, more focus is needed to optimizing the extraction process of $\beta$-carotene.

\subsubsection{By-products of tomato}

Tomato (Lycopersicon esculentum) contains large amounts carotenoids (around 90-180 mg per $\mathrm{kg}$ fresh weight), mainly in the form of lycopene (80-90\% of total carotenoids), which accumulates during the final stages of fruit ripening and provides the intense red fruit colour, and $\beta$-carotene, which accounts for around $2-3 \%$ 


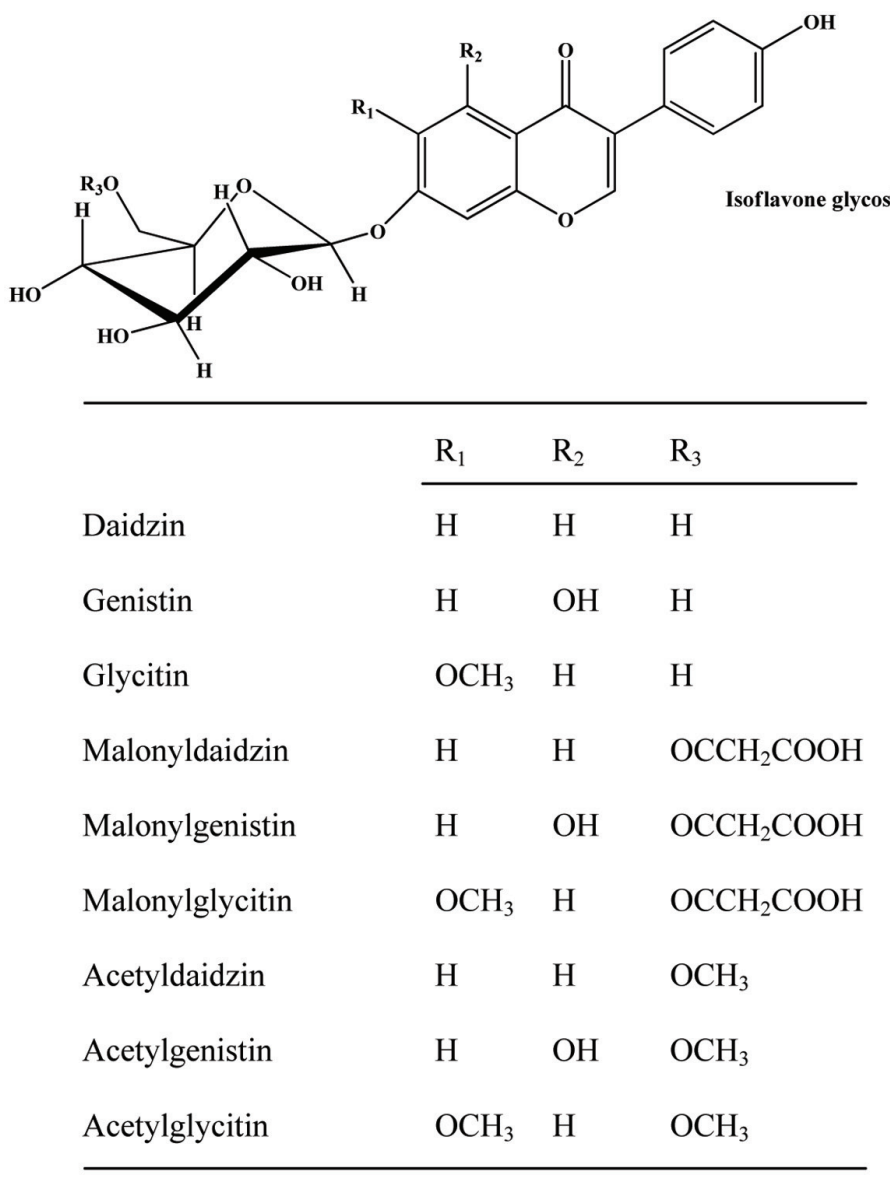

Figure 38. Structure of soy isoflavone glucosides.

of total carotenoids (Dumas et al., 2003; Riggi and Avola, 2008; Shi and LeMaguer, 2000).

The major by-product generated during tomato processing are tomato pomace, consists of the dried and crushed skins and seeds of the fruit (Avelino et al., 1997). The seeds contribute approximately $10 \%$ of the fruit and $60 \%$ of the total waste in weight basis and large quantity of carotenoids is also lost as waste in tomato processing (Baysal et al., 2000). Tomato processing waste (skin and seeds) contains high amounts of bioactive compounds such as $\beta$-carotene $(32.6 \mathrm{mg} / \mathrm{kg})$, ascorbic acids $(111.89 \mathrm{mg} / \mathrm{kg})$, and lycopene $(174$ $\mathrm{mg} / \mathrm{kg}$ ) (Nour et al., 2015). Baysal et al. (2000) studied the supercritical $\mathrm{CO}_{2}$ extraction of lycopene and $\beta$-carotene from by-product of tomato paste and found that recovery was up to $50 \%$ when ethanol was added. Another study showed that enzymatic treatment of tomato marc enhanced lycopene extractability (Böhm et al., 2000).

Lycopene is the principal carotenoid causing the characteristic red hue of tomatoes. Most of the lycopene is associated with the water-insoluble fraction and the skin (Sharma and Maguer, 1996). Tomato skin contains high levels of lycopene compared to the pulp and seeds (George et al., 2004; Toor and Savage 2005; Wandawi et al., 1985). Therefore, skin extracts are especially rich in lycopene. George et al. (2004) studied the antioxidant components in 12 field grown tomato genotypes and reported that, on average, the tomato skin had 2.5 times higher lycopene levels than the pulp. According their findings, the extent of variation was 4.8 to $14.1 \mathrm{mg} / 100 \mathrm{~g}$ in peels and 2.0 to $6.9 \mathrm{mg} / 100 \mathrm{~g}$ in pulp on fresh weight basis. A similar observation has been made in previous study by Al-Wandawi et al. (1985) who reported a three times higher lycopene content in the peel fraction.

\subsection{Betalains}

Beetroot is the major dietary source of betalains. Like anthocyanins, betalains play important roles both in plant physiology, visual attraction for pollinators and seed dispersers. They are usually dissolved in the vacuolar sap as bi-anions (Wyler, 1969). Structurally, the betaxanthins are condensation products of betalamic acid and various amino compounds, whereas the betacyanins are characterized by a cyclo-dopa structure with additional substitutions through varying glycosylation and acylation patterns at C-5 or C-6. Their most common representative is betanidin $5-O-\beta$-glucoside (betanin) from red beet (Beta vulgaris ssp.) (Stintzing and Carle, 2004). Therefore, the presence of aromatic amino compound moieties both in betacyanins and betaxanthins exhibit the antioxidant properties by stabilizing free radicals in plant tissues. Dearth of literature available on in vivo and in vitro studies of antioxidant and antiradical potency of betalains.

\subsubsection{By-products of beetroot}

The pomace from the beetroot juice industry accounting for 15 $30 \%$ of the raw material (Otto and Sulc, 2001) is disposed as feed 


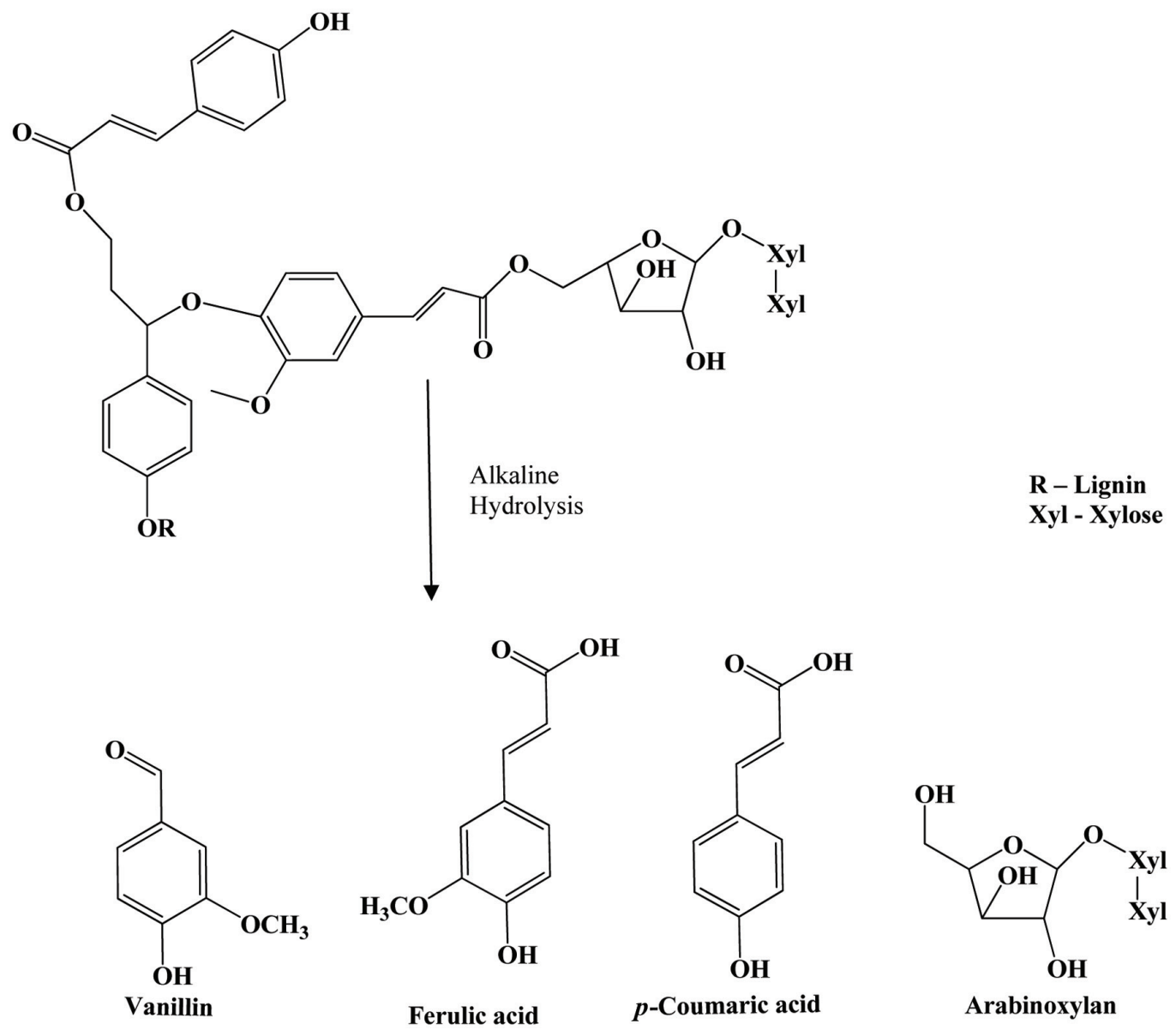

Figure 39. Cleavage of lignin/phenolics-carbohydrate complexes in flax shives with alkali. Source: Adapted from Buranov and Mazza, (2008).

or manure. Peel, carried the main portion of betalains with up to $54 \%$, being lower in crown (32\%) and flesh (14\%) (Kujala et al., 2000). Whereas the coloured fraction consisted of betalains (betacyanins and betaxanthins) such as betanin, prebetanin, isobetanin, neobetanin, vulgaxanthin I, vulgaxanthin II, and indicaxanthin, the phenolic portion of the peel showed $l$-tryptophan, $p$-coumaric and ferulic acids, as well as cyclodopa glucoside derivatives (Kujala et al., 2001). Vulić et al. (2014) found three bioactive compounds, namely betanin, isobetanin, and vulgaxanthin I in beetroot pomace extract and some phenolics, including catechin, rutin, and caffeic acid.

Betalains and anthocyanins are mutually exclusive in their natural occurrence, but other flavonoids are often produced in betalainbearing plants (Stafford, 1994). The total phenolics distribution in red beetroot (Beta vulgaris) root appears to be quite similar to that reported for the potato. Kujala et al. (2001) showed that total phenolics decreased in the order peel (50\%), crown (37\%), and flesh $(13 \%)$. Therefore, the exploitation of peel and pomace for phenolics and betalains is a real need. Temperature- and $\mathrm{pH}$-dependent in vitro antiradical activities have been reported for betanin and betanidin carrying phenolic hydroxyl groups (Escribano et al., 1998; Pedreno and Escribano, 2001), thus being more efficient than the betaxanthins vulgaxanthin I and II (Escribano et al., 1998). However, betaxanthins that bear phenolic structures in their amino acid moiety, e.g. portulacaxanthin II, miraxanthin III, miraxanthin $\mathrm{V}$ and dopaxanthin (Stintzing et al., 2001) may act in a similar way.
Little is known about the in vivo absorption of betalains. Betacyanins were demonstrated to be strong antioxidants in various model systems, and their positive charge may increase their affinity to biological membranes, which are the preferred targets of oxidation (Kanner et al., 2001). Literature data imply a low rate of betalain absorption, and a critical concentration for the bioactivity of these compounds in human plasma has yet to be established.

\section{Bioavailability of phenolic compounds}

Bioavailability refers to the proportion of an active moiety, which enters systemic circulation unchanged, thereby accessing the site of action, after a particular route of administration (Ververidis et al., 2007). Potential health effects (sec 12) of many phenolic compounds have been extensively investigated using in vitro methods and some have been supported by in vivo studies (Williamson and Manach, 2005; Shahidi and Ambigaipalan, 2015). Although the health effects of phenolics depend on their respective intake, there is a need to assess whether in vitro effects could be obtained in vivo, because of the bioaccessibility and bioavailability of phenolics largely differs reflected by the actual human pharmacokinetic studies of phenolics (Manach et al., 2005a). Bioavailability of these compounds varies considerably according to their structural diversity and is also affected by various external factors such as the food matrix and the health condition of the host. In addition to 
<smiles>O=C(O[C@H]1Cc2c(O)cc(O)cc2O[C@@H]1c1cc(O)c(O)c(O)c1)c1cc(O)c(O)c(O)c1</smiles>

Figure 40. Structure of (-)-epigallocatechin-3-O-gallate tea catechins.

structural diversity (physicochemical properties) of the molecule, enzymate and microbial-mediated biotransformation, the limited expression of phenolic transporters and rapid detoxification process also limit the bioavailability of dietary phenols (Lambert and Yang, 2003; Manach et al., 2004; Scalbert et al., 2002; Shahidi and Peng, 2018). Therefore, most abundant dietary phenolics do not necessarily reflect a highest concentration of active metabolites functioning in target tissues, the phenolic structures with the highest in vitro bioactivity also may not accurately reflect an actual in vivo bioactive property. The bioavailability involved with digestion, absorption, metabolism, distribution, transporting, excretion, and colonic fermentation have to be considered.

Most of the polyphenols, however, are present in food in the form of esters, glycosides, or polymers which is difficult to pass through enterocytes depending on no matter paracellular transport or intracellular transport (passive transport, active transport, and facilitated transport), thus must be hydrolyzed by intestinal enzymes (lactase-phlorizin hydrolase and cinnamoyl esterases) or catabolized by the colonic microbiota before absorption (Shahidi and Peng, 2018). Many of the studies have investigated the kinetics and extent of polyphenol absorption by measuring plasma concentrations and/or urinary excretion among adults after the ingestion of a single dose of polyphenol, provided as pure compound, plant extract, or whole food/beverage. Detailed reviews on the bioavailability of polyphenols in humans have been published (Crozier et al., 2009; Karakaya, 2004; Manach et al., 2005a; Yang et al., 2008;). This review will focus on intestinal absorption and metabolism of selected dietary phenols in humans, from different dietary sources.

\subsection{Flavonoids}

According to the rule-of-five prediction model, compounds with relative molecular mass of $>500 \mathrm{Da}$ and prone to form hydrogen bond interactions are generally unable to cross biological membranes by passive diffusion (Lipinski et al., 2001), and thus most of dietary flavonoids with low lipophilicity have less chance to be passively absorbed at the gastrointestinal (GI) level. Flavonoids and their metabolites may be absorbed in the large intestine but will be subjected to the anaerobic degradation including peripheral and central aromatic catabolism of the colonic microflora, which decomposes the heterocyclic ring of backbones, as well as other ester/ether bonds with the conjugating moieties (Shahidi and Peng, 2018). Apart from colonic catabolism, the conjugation reactions occurring in human cells such as enterocytes, hepatocytes, and neurocytes are very efficient and rapid so that the predominated forms of flavonoids in plasma and urine were found as various conjugates (Shahidi and Peng, 2018). Thus it is difficult to detect flavonoid aglycones in plasma, because they occur mostly at below the detection limit of the analytical methods used (Hollman, 2004). However, most studies have showed that bioavailability of flavonoid is often $<1 \%$ of the administered dose (Manach et al., 2005a).

\subsubsection{Flavonols}

Flavonols, especially quercetin, are widely distributed in plant foods. In general, studies have demonstrated that bioavailability of quercetin glucosides from onions is superior to other sources of quercetin glycoside. According to Hollman et al. (1997), bioavailability of various quercetin glycosides from apples and of pure quercetin rutinoside is only $30 \%$ of that from onions. When compared to other major dietary sources, namely wine and black tea, the plasma concentration of quercetin after wine consumption is lower than that after ingesting onions and not different from that of black tea, while urinary excretion of quercetin after wine intake did not differ from that of onions, but was higher than that after drinking black tea (de Vries et al., 2001). In addition, the same study showed that the sum of the three flavonols, quercetin, kaempferol and isorhamnetin, was lowest in both plasma and urine after red wine consumption. Based on this finding, the authors reported that the bioavailability of flavonols from red wine was not better than that from onions or black tea in the diet because one glass $(125 \mathrm{~mL})$ of red wine provides the lower amounts of available flavonols than one portion of onions $(15 \mathrm{~g})$ or one glass of tea $(125 \mathrm{~mL})$. The fact that certain flavonol glycosides are absorbed more rapidly than others suggests the importance of the attached sugar moiety on their rate of absorption (Hollman et al., 1999). Further, the same authors suggested that quercetin glucoside is absorbed by active/facilitated transportation to the small intestine by several transporters including 3 glucose transporters (GLUT 1/2/4) and sodium-dependent glucose transporter (SGLT 1) summarized by latter studies (Shahidi and Peng, 2018), whereas quercetin rutinoside is absorbed from the colon after deglycosylation. Olthof et al. (2000) investigated whether the position of the glucose moiety affected quercetin bioavailability in humans and suggested that the quercetin glucosides were rapidly absorbed irrespective of the position of the glucose moiety. Major conjugates of quercetin after onion supplementation were the 3 '-sulfate, the 3'-methoxy-3-glucuronide, and the 3-glucuronide (Day et al., 2001).

\subsubsection{Flavonone}

The bioavailability of flavanone conjugates and their aglycones after the intake of orange juice, grapefruit juice or pure compounds in humans has been reported by a number of studies (Ameer et al., 1996; Erlund et al., 2001; Fuhr and Kummert, 1995; Gardana et al., 2007; Ishii et al., 2000; Kanaze et al., 2007; Lee and Reidenberg, 1998; Manach et al., 2003; Nielsen et al., 2006). In most analyses, researchers collected plasma and urine after ingestion and cleaved putative hesperetin and naringenin glucuronide conjugates by 
treatment with glucuronidase before analysis of the released aglycones. It was shown that absorption of flavanones occurs in the colon due to their binding to a rutinose or neohesperidose moiety in food sources. Mullen et al. (2008) used HPLC-MS to analyse flavanone bioavailability in humans after the intake of orange juice and reported that there was a substantial metabolism of orange juice flavanone in the gastrointestinal tract, most notably the large intestine, where colonic bacteria play a major role. The specific colonic biotransformation pattern of flavonones and known related bacterial species were specifically described by other reviews (Mosele et al., 2015; Sánchez-Patán et al., 2012; Shahidi and Peng, 2018). When compared to other flavonoids, bioavailability of flavanone seems to be equivalent to that of flavonols and flavanols and the elimination of flavanones in urine is not as rapid as that of catechins, but slightly faster than that of quercetin (Manach et al., 2003).

\subsubsection{Flavan-3-ols}

As outlined earlier, tea, chocolate, apples, pears, grapes, and red wines are rich sources of flavan-3-ols. Bioavailability of flavan3-ols (proanthocyanidins and tea catechins) is very poor due to their limited absorption and rapid elimination (Manach et al., 2005a). Absorption of catechins is believed to occur through the small intestine because bacterial degradation within the colon is hypothesized to break down the flavonoids into smaller phenolic acids including hydroxyphenyl acetic acid, hydroxyphenylpropionic acid, phenylpropionic acid, and hydroxyphenylvaleric acid (Déprez et al., 2000; Rechner et al., 2004). Catechins are metabolized and circulated as sulfated, methylated, or glucuronidated derivatives, as well as various phenolic catabolites absorbed in the colon (Lee et al., 2002; Meng et al., 2002; Piskula and Terao, 1998; Sun et al., 2017; Shahidi and Peng, 2018).

\subsubsection{Isoflavones}

Isoflavones exhibit the highest bioavailability of all subclasses of dietary flavonoids with urinary excretion of metabolites typically at $20-50 \%$ of their intake level (Donovan et al., 2006; Hollman, 2004; Manach et al., 2005a). Their high bioavailiability is mainly attributed to the relatively moderate lipophlicity of most of isoflavone aglycones which is in accord with Lipinski's rule of five that the molecules with octanol-water partition coefficient log P ranging from $2-5 \%$ have better bioavailability (Lipinski et al., 2001). Isoflavones such as daidzein, genistein, $O$-desmethylangolensin and equol are the main flavonoids and their metabolites in an ordinary Japanese diet and are found mainly in the conjugated form in the plasma samples of Japanese men (Karakaya, 2004), but the absorption of isoflavones differs between Japanese and Americans because intestinal microflora, dietary habits, and ethnic background all have an effect (Zubik and Meydani, 2003). However, there is still controversy about the bioavailability of aglycones vs glucosides. The intestinal absorption of most isoflavones is thought to require the release of aglycones from glucoside conjugates (Manach et al., 2004). Zheng et al. (2004) demostrated that isoflavone aglycones were absorbed more rapidly than glucosides as reflected in plasma $3 \mathrm{~h}$ after feeding and did not differ after $24 \mathrm{~h}$. Based on this observation they suggested that the glucoside moiety prevented absorption of the isoflavone, and therefore absorption of isoflavones from their glucosidic form, depended, at least in part, on lower gut microbes which cleave the glucose moiety. Thus, the intestinal absorption of glucosides is thought to be delayed (Izumi et al., 2000; Zheng et al., 2004). However, Richelle et al. (2002) and Zubik et al. (2003) reported that the absorbability of isoflavones does not differ when ingested as either corresponding aglycones or glucosides.

In a study by Rüfer et al. (2008), seven male volunteers were given either pure daidzein or pure daidzein-7-O-glucoside, both at a dose of $3.9 \mu \mathrm{mol} / \mathrm{kg}$ body weight. They demonstrated that the plasma Cmax, at ca. 8-9 h, was three to six times longer after consumption of the glucoside, which is dominant in soy compared with the aglycone, the main component in fermented soy products. The bioavailability reported in this study contrasts markedly with the results of Izumi et al. (2000) and Kano et al. (2006). Izumi et al. (2000) investigated the tablets containing a crude preparation of soy saponins and either daidzein and genistein aglycone or their mixed glycosides, which were given to eight volunteers weighing $51-87 \mathrm{~kg}$ at doses of $0.11(30 \mathrm{mg}$ aglycone and $50 \mathrm{mg}$ glycosides) and 1.7 (450 mg aglycone and $760 \mathrm{mg}$ glycosides) mmol. They found that at the higher dose, the isoflavone aglycone mixture produced plasma Cmax concentrations up to five times higher than diadzein and genistein glycosides. The Tmax in this study was ca. $4 \mathrm{~h}$, which is much shorter than the $8-9 \mathrm{~h}$ reported by Rüfer et al. (2008). Crozier et al. (2009) suggested that saponins might play a role in these differences. According to the study of Kano et al. (2006) in humans, isoflavone aglycones are absorbed faster and in greater amounts than glucosides when ingested in the form of a beverage like soymilk. On the other hand, the colonic catabolism and in vivo metabolism of isoflavonoids greatly alter their absorption and distribution. Along with the deglycosidation in colon, more biotransformations may happen. For example, equol, one of the colonic catabolites of isoflavone aglycones, possesses a much higher lipophilicity and less hydrogen donor ability which may result in its better absorption (Setchel et al., 2002). Besides equol, various simple phenolic acids derived by cleavage of heterocyclic ring of isoflavones also affect the actual form of isoflavones occurring in the plasma rather than only the original isoflavones and their conjugates which has been discussed in other publications (Braune and Blaut, 2016; Gaya et al., 2016; Shahidi and Peng, 2018).

\subsubsection{Anthocyanins}

Studies have shown that mainly cyanidin-based anthocyanins conjugated with glucose are absorbed like some other phenolics frequently found in the diet such as catechin and quercetin glycosides (McGhie et al., 2003). However, there are several important differences in the absorption and the bioavailability of anthocyanins when compared with other flavonoids (McGhie and Walton, 2007). At dietary relevant doses, it seems that essentially all of the absorbed catechin or quercetin was present as metabolites, that is, conjugates of glucuronic acid or sulfate or methyl group (Donovan et al., 1999; Sesink et al., 2001). In contrast, anthocyanins were also absorbed and excreted largely unmetabolized in both rats and humans. Therefore, they do not appear to undergo extensive metabolism of the parent glycosides to glucurono, sulpho or, methylderivatives (Cao et al., 2000; Cooney et al., 2004; Felgines et al., 2002; Ichiyanagi et al., 2005; Matsumoto et al., 2001; McGhie et al., 2003; Milbury et al., 2002; Miyazawa et al.,1999; Murkovic et al., 2001; Tsuda et al., 1999; Wu et al., 2002). This complex array of information about anthocyanin bioavailability in humans and animals revealed that the determinants of absorption and excretion are influenced by the nature of the sugar moiety and the structure of the anthocyanidin aglycone (Prior and $\mathrm{Wu}, 2006$; Wu et al., 2005). Anthocyanins exist in the cationic form, but their stability is 
dependent on $\mathrm{pH}$. With the $\mathrm{pH}$ change from acid to alkaline during the digestion and colonic fermentation, the backbone of anthocyanins would change from flavylium to be pseudobase or chalcone (Aura et al., 2005, Hidalgo et al., 2012). Therefore, pH conditions may contribute to the difference of absorption, transportation and metabolism between anthocyanins and other phenolics.

\subsection{Tannins}

Serrano et al. (2009) have reported the bioavailability of tannins in a detailed review. Very few studies on bioavailability of polymeric tannins in humans have been reported and the results are controversial. However, theoretically, polymeric tannins could not penetrate the enterocytes and be absorbed without any decomposition process. Most studies in humans have been conducted after the intake of pomegranate juice. It has been reported that ellagitannins release ellagic acid upon in vivo hydrolysis (Daniel et al., 1991), and pure ellagic acid has poor bioavailability compared with ellagitannins (Lei et al., 2003). Seeram et al. (2006) demonstrated that consuming $180 \mathrm{~mL}$ of pomegranate juice concentrate was associated with maximum plasma concentration $(0.06 \mu \mathrm{mol} / \mathrm{L})$ of ellagic acid after $1 \mathrm{~h}$; ellagic acid metabolites, total urolithin A of 0.14 $\mu \mathrm{mol} / \mathrm{L}$ and total urolithin B of 0.01 at $6 \mathrm{~h}$. Cerda et al. (2004) reported that neither punicalagin nor ellagic acid were detected in plasma or urine following daily intake of $1 \mathrm{~L}$ of pomegranate juice containing $4.37 \mathrm{~g}$ punicalagins over 5 days. They detected microbial ellagitannin-derived metabolites, urolithins in fasting plasma and urine. Urolithins, arise from biotransformation by the intestinal microflora on ellagic acid, which is derived by degradation of ellagitannin by human microbiota via hydrolysis (Cerda et al., 2005a). After consumption of other ellagitannin-containing foodstuffs' namely strawberries, raspberries, walnuts, and oak-aged wine metabolite excretion (urolithin B) ranged from 2.8 to $16.6 \%$ of the ingested ellagitannins.

The study of Cerda et al. (2005b) showed that metabolite excretion after the consumption of common strawberries, raspberries, walnuts, and oak-aged wine ranged from 2.8 to $16.6 \%$ of the injested ellagitannins. These findings suggest that ellagitannin metabolism in humans depends on the colonic microflora of each individual.

\subsection{Phenolic acids}

Zhao and Moghadasian (2009) have reviewed the in vivo and in vitro investigation on hydroxycinnamates. Caffeic acid, ferulic acid and as well as the two groups of chlorogenic acids (caffeoylquinic acids and dicaffeoylquinic acids) were mainly reported in bioavailable studies. Relatively, few bioavailability data have been reported on hydroxybenzoic acids due to their limited distribution in foods (Shahidi and Naczk, 2003; Fernandez de Simon et al., 1992; Tomas-Barberan and Clifford, 2000). Most studies have been on the absorption and metabolism of gallic acid and have shown that they are well absorbed (Lafay and Gil-Izquierdo, 2008). The compound 4-O-methylgallic acid has been identified as a primary metabolite of gallic acid in human plasma and urine (Mohsen et al., 2002; Shahrzad and Bitsch, 1998).

As discussed in section 2.2, the major representative of hydroxycinnamic acids in food is caffeic acid. It occurs largely conjugated with quinic acid, as in chlorogenic acid. In experiments with volunteers who had undergone colonic ablation, chlorogenic acid absorption was also three times lower than that of caffeic acid (Olthof et al., 2001). A similar observation has been reported by Lafay et al. (2006) in a rat model. Chlorogenic acid has been identified by several authors in urine after the ingestion of coffee or pure chlorogenic acid, suggesting its direct absorption in the gut (Cremin et al., 2001; Gonthier et al., 2003; Ito, et al., 2005; Olthof et al., 2001, 2003). However, in many studies, chlorogenic acid was not detected in the plasma or bile (Azuma et al., 2000; Booth et al., 1957; Bourne and Rice-Evans, 1998; Choudhury et al., 1999; Lafay et al., 2006; Nardini et al., 2002). Furthermore, Farah et al. (2008) confirmed that caffeoylquinic acids and dicaffeoylquinic acids, which are major chlorogenic acid compounds in coffee, are absorbed in the human body, being differentially absorbed and/ or metabolized throughout the whole gastrointestinal tract. Their study also evidenced that urine is not a major excretion pathway of intact chlorogenic acids and their metabolites and identified sinapic, gallic, $p$-hydroxybenzoic, and dihydrocaffeic acids as major urinary metabolites of chlorogenic acid in humans. In addition, their study showed that the main chlorogenic acids present in the green coffee matrix were highly bioavailable in humans.

Ferulic acid, quantified as urinary excretion, shows low to high bioavailability (0.4-98\%) depending on the food source (Anson et al., 2009; Bourne et al., 2000; Heleno et al., 2015; Karakaya, 2004; Manach, et al., 2005a). For instance, in wheat grain, most of ferulic acid is bound to arabinoxylans and other indigestible polysaccharides restricting its release in the small intestine (Anson et al., 2009). Kern et al. (2003) have also shown that only a small proportion $(<3 \%)$ of ferulic acid and sinapic acid have been recovered in the urine in relation to the total amount of ingested commercial high-bran breakfast cereal. The bioavailability of ferulic acid was somewhat higher from other food matrices such as tomato (1125\%; Bourne and Rice-Evans, 1998), rye bread, $(<28 \%$; Harder et al., 2004), or beer (19-98\%; Bourne et al., 2000). Therefore, appropriate processing techniques should be identified to increase free ferulic acid in the cereal matrix in order to improve its bioavailability. However, the exact mechanism for the absorption of hydroxycinnamic acids is not clear. Konishi et al. (2003, 2004) showed that hydroxycinnamic acids might be transported across the intestinal epithelial cells by monocarboxylic acid transporters. Studies of Poquet et al. (2008), Adam et al. (2002) and Zhao et al. (2004) and (2008) suggest that a passive diffusion mechanism may be involved in the absorption of hydroxycinnamic acids. The metabolism of hydroxycinnamic acids may occur in the liver, intestinal mucosa, kidney, and/or by intestinal microflora (Chesson et al., 1999; Zhao and Moghadasian 2008). During metabolism, hydroxycinnamic acids may undergo a number of enzymatic reactions including dehydroxylation, demethylation, dehydrogenation, hydrogenation, $O$-methylation, sulphation, glucuronization, GSH conjugation, and/or glycination (Zhao and Moghadasian 2009). As for colonic fermentation, the $\alpha / \beta$-oxidation usually breaks the $\mathrm{C}-\mathrm{C}$ covalent bonds of the side chain of hydroxycinnamic acids, leading to the shortening of the side chains with the formation of a carboxyl group. Meanwhile, dehydroxylation and decarboxylation also degrade hydroxycinnamic acids into simpler phenols, which may contribute a higher absorption of catabolites of hydroxycinnamic acids in the colon (Knockaert et al., 2012; Ludwig et al., 2013). Conjugation of phenolic acids, the colonic metabolites of flavonoids, also seems to occur less efficiently, with conjugation percentages ranging from 13 to $100 \%$, depending on the type of phenolic acid involved (Olthof et al., 2003).

\subsection{Tyrosol/hydroxytyrosol}

Olive oil and its production wastes are the major sources of oleuropein, hydroxytyrosol and tyrosol. Several human studies have 
shown that olive oil phenols are bioavailable (Caruso et al., 2001; Miró-Casas et al., 2001, 2003a, 2003b; Visioli et al., 2000, 2003; Vissers et al., 2002). In a human ileostomy study, it was shown that up to $66 \%$ of the ingested olive oil phenols were absorbed in the small intestine (Vissers et al., 2002).

Urinary recoveries as high as $80 \%$ of the ingested amounts of hydroxytyrosol have been reported in humans (Miró-Casas et al., 2003a, 2003b), with considerably lower excretions of tyrosol (20-25\% of the initial doses) (Miró-Casas et al., 2001). Over $90 \%$ of the urinary metabolites were conjugates (Caruso et al., 2001; Miró-Casas et al., 2001, 2003a, 2003b; Visioli et al., 2000; Vissers et al., 2002), mainly glucuronidated metabolites, yet free phenols and methyl conjugates, with or without glucuronidation, were also excreted in the human urine. Along with the phase II metabolism, the phase I also influences the bioavailability of tyrosol. Actually, tyrosol can be transformed to hydroxytyrosol depending on the hydroxylation of CYP-450, which is proven to be CYP2A6/CY$\mathrm{P} 2 \mathrm{D} 6$. In addition, enthanolin the wine could significantly increase the bioavailability of tyrosol (Rodríguez-Morató et al., 2016a). More information about different analytical approaches that have been developed to measure the plasma and urinary levels of both hydroxytyrosol and tyrosol and the details of their absorption, distribution, metabolism and excretion processes have been provided by Rodríguez-Morató et al. (2016b).

\subsection{Stilbene (trans-resveratrol)}

Oral absorption of trans-resveratrol is high but its bioavailability is very low because it is rapidly and extensively biotransformed by first-pass metabolism (Walle et al., 2004; Wenzel and Somoza 2005). Meng et al. (2004) reported that free and conjugated resveratrol were detectable in human plasma after a high-dose administration of the pure compound $(0.5-1 \mathrm{mg} / \mathrm{kg})$ to fasting subjects. However, it was not detectable in human plasma after its ingestion from grape juice (0.6-1.8 $\mathrm{mg}$ of total resveratrol). These results suggested the lower bioavailability of resveratrol glycosides in grape juice in comparison with its pure aglycone and/or possible influence of the matrix sugar content on resveratrol bioavailability. Walle et al. (2004) demonstrated that the absorption of a dietary relevant $25 \mathrm{mg}$ oral dose of resveratrol was at least $70 \%$ and that in vivo the molecule was metabolized not only through sulphate and glucuronic acid conjugation of the phenolic groups but also through hydrogenation of the aliphatic double bond, probably due to the intestinal microflora. Vitaglione et al. (2005) showed that trans-resveratrol in humans can be actually absorbed after moderate wine consumption ( 300 or $600 \mathrm{ml}$ ). In this study, 25 subjects were involved and it was clearly demonstrated that resveratrol absorption after wine consumption is highly variable. Further, the same study revealed that trans-resveratrol was found in the serum of roughly half of the subjects participating in the experiments, in the free or in the glucuronidated form and in very different concentrations. In the colon, the double bond of $\mathrm{C} 2$ structure between two aromatic rings of stilbene would be hydrogenated. Meanwhile, hydroxylation, dehydroxylation, and various conjugation would also modify stilbene aglycone into different products. Therefore, much more derivatives of stilbene were found in actual human pharmacokinetic study (Rotches-Ribalta et al., 2012a).

\section{Health benefits of phenolic compounds}

As discussed earlier, the by-products of the agri-food industries constitute an interesting source of phenolic compounds. Potential health benefits of many phytochemicals have been demonstrated by in vitro and in vivo studies and excellent reviews and works on this topic have been presented and published (Biedrzycka and Amarowicz, 2008; Boris, 1996; Bravo, 1998; Chandrasekara and Shahidi, 2011; Dillard and German, 2000; Halliwell 1996; Heinecke 1997; Hertog et al., 1993a,b; Huang et al., 1992; Isanga and Zhang, 2007; Lakhanpal and Rai, 2007; Lampe, 1999; Nichenametla et al., 2006; Oomah and Mazza, 1999; Shahidi and Chanderasekara, 2013; Shahidi, 1997, 2000 and 2007; Shahidi and Ho 2007; Stintzing and Carle, 2004; Weichselbaum et al., 2010; Yang, et al., 2008). Furthermore, Fraga et al. (2010) reported the possible mechanisms involved in rendering the health benefits of polyphenols. However, this contribution mainly reviews the studies on health benefits related to the phenolic constituents extracted from by-products of agri-food industry.

The health benefits of phenolics are primarily in several areas, including cancer, atherosclerosis and other cardiovascular disease, the aging process and immune response-enhancing effect, diabetes, mental health, spasmodic conditions, ulcers, and among others (Dillard and German, 2000; Shahidi 2007). Oxidation of low-density lipoprotein (LDL) is a key event leading to the formation of atherosclerotic plaques (de Vries et al., 1998). It is generally reactive oxygen species (ROS) as well as enzymes, such as lipoxygenase and myeloperoxidase, are involved in the oxidation of LDL (Heinecke, 1997; Morton et al., 2000). Some studies have shown support for the role of dietary phenolics against cardiovascular disease due to their antioxidant effects (Hertog et al., 1993a; Nardini et al., 1995; Renaud and Lorgeril, 1992). Flavonoids have also been reported to exert positive effects on the cardiovascular system through modulation of the nitric oxide synthase system (Fitzpatrick et al., 1998; Park et al., 2000; Visioli et al., 1998). However, not all epidemiological studies have found a protective effect of dietary phenolic compounds against heart disease (Rimm et al., 1996; Hertog et al., 1997).

Phenolic compounds in nuts and their by-products, mainly ellagic acid, resveratrol, and flavonoids provide protection against harmful free radicals and are known to reduce the risk of coronary heart disease and atherosclerosis. Cashew nut shell phenolics are used in traditional medicine as anti-inflammatory, antidiarrheal, anti-asthmatic and depurative agents. Previous studies have confirmed their biological properties, including antibacterial, anti-inflammatory, antiproliferative, antioxidant and enzymatic inhibitory activities (de Sousa Leite et al., 2016; de Souza et al., 2018). Chen et al. (2005) reported on the extracted almond skin flavonoids and demonstrated their antioxidant capacity in vitro. These flavonoids were found to be bioavailable and acted synergistically with vitamins C and $\mathrm{E}$ to protect low-density lipoprotein (LDL) cholesterol against oxidation in hamsters and humans. Walnut skin phenolics also have been shown to inhibit oxidation of low-density lipoprotein (LDL) cholesterol, a key step in atherogenesis (Anderson et al., 2001).

Phenolic compounds in red wine and wine by-products, particularly the anthocyanins, hydroxycinnamic acids, proanthocyanidins other flavonoids and stilbenes, are hypothesized to be the beneficial components responsible for inhibiting the oxidation of lowdensity lipoprotein (LDL) and thus, providing protection against atherosclerosis (Frankel et al., 1993; Tebib et al., 1994; Yamakoshi et al., 1999).

Resveratrol has been reported to exhibit antioxidant (Soares et al., 2003; Oh and Shahidi, 2017; Oh and Shahidi, 2018), cardioprotective (Chen and Pace-Asciak, 1996; Wang et al., 2002) and anti-inflammatory properties (Subbaramaiah, et al., 1998; Martin et al., 2004). Other studies have shown that resveratrol can inhibit cellular events associated with carcinogenesis-cancer chemo- 
preventive activity including tumour initiation, promotion and progression (Jian et al., 1999). In vivo studies have indicated that resveratrol has anticancer effects against intestinal (Ziegler et al., 2004), colon (Tessitore, et al., 2000) and skin cancer (Nichenametla et al., 2006). In contrast, other studies reported no anticancer effect and even carcinogenic properties at high dosage levels of resveratrol (Bove et al., 2002; Sato et al., 2003). Furthermore, Scalbert and Williamson (2000) noted that the very low concentration of resveratrol in red wine $(0.3$ to $2.0 \mathrm{mg} / \mathrm{L})$ (Frankel et al., 1995) makes the attribution of protective effects to this compound unlikely. In addition to that, it acts as modulator of lipoprotein metabolism (Goldberg et al., 1995; Soleas et al., 1997), inhibitor of platelet aggregation (Bertelli et al., 1995; Pace-Asciak et al., 1995) and vaso-relaxing agent (Chen, and Pace-Asciak, 1996; Jager and Nguyen-Duong, 1999).

Proanthocyanidin-rich extracts from grape seeds display anticataract activity in rats (Yamakoshi et al., 2002). Since oxidative stress is responsible for cataract formation (Taylor et al., 1995) the inhibition of cataract progression was suggested to be due to the antioxidant activity of the grape seed proanthocyanidins (Yamakoshi et al., 2002). In other studies, grape seed extracts were found to possess antiulcer properties in rats (Saito et al., 1998). It was suggested that the antiulcer property was due to the radical scavenging activity of the grape seed proanthocyanidins and their ability to bind proteins. Studies in animal models show that procyanidins from grape seeds inhibit chemically induced lipid peroxidation, DNA fragmentation, and subsequent apoptosis in a dose-dependent manner (Bagchi et al., 1998). Rats fed with a diet enhanced with procyanidins had higher plasma antioxidant activity and their hearts were less susceptible to ischemia/perfusion damage induced by iron and copper ions (Facino et al., 1999). Quiñones et al. (2013) reported that ingestion of procyanidins had an antihypertensive effect in spontaneously hypertensive male rats with a significant decrease in systolic and diastolic blood pressure. The antihypertensive effect of procyanidins for spontaneously hypertensive rats was dose-dependent up to $375 \mathrm{mg} / \mathrm{kg}$ (maximum decrease $6 \mathrm{~h}$ post-administration), although the procyanidins-fed group did not show any effect on blood pressure of Wistar-Kyoto rats (Quiñones et al., 2013). Besides, procyanidins could reduce not only animal weight and plasma lipid but also inflammation tumor necrosis factor- $\alpha$ (TNF- $\alpha$ ) and C-reactive protein (CRP) (Terra et al., 2011; Caimari et al., 2013). More recently, Shi et al. (2019) fed high-fat diet to one hundred male C57BL/6 mice with/ without proanthocyanidins extracts for 180 days. It was found that the proanthocyanidins extracts could significantly decrease the weight gain, serum levels of triacylglycerols, total cholesterol, and LDL cholesterol in the high-fat diet group but increased their HDL cholesterol level. The differential effect of grape seed extract and its active constituent procyanidin B2 3,3"-di-O-gallate against prostate cancer stem cells was also investigated by Tyagi (2019).

Human studies also show that diet rich in procyanidins decrease/inhibit lipid peroxidation of LDL cholesterol and increase free radical scavenging capacity (Natella et al., 2002; Fuhrman et al., 1995). Procyanidins were reported to selectively inhibit protein kinase C (Takahashi et al., 2000) and intensively promote hair growth by enhancing proliferation of mouse hair epithelial cells in vitro and activating hair follicle growth in vivo (Takahashi et al., 1999). Procyanidins were also found to slow the proliferation and decrease apoptosis of pancreas $\beta$-cells induced by hydrogen peroxide and promote proliferation of normal pancreas $\beta$-cells (Zhong, 2003).

Berries peel and pomace are the good source of anthocyanins. The anthocyanins extracted from bilberry (Vaccinium myrtillus) juice have been reported to protect the vascular system in vivo by increasing the permeability of capillary blood vessels (Azar et al., 1987). Anthocyanins have been shown to have stimulatory effect on the secretion of tumour necrosis factor- $\alpha$ in macrophages in vitro (Wang and Mazza, 2002) which are responsible for cytostatic and cytotoxic activities on malignant cells (Camussi et al., 1991). Inhibitory effects of apple polyphenols and related compounds on cariogenicity of streptococci suggest their possible application in dentifrices (Yanagida et al., 2000).

Dried citrus fruit (Citrus reticulata) peels are widely used as remedies to alleviate coughs and reduce phlegm in the respiratory tract in Chinese medicine (Ho and Lin, 2008; Ou, 1999).Citrus flavonoids attract considerable attention, for their significant biological activities, including anti-inflammatory, anticancer, and antiatherogenic activities (Tripoli et al., 2007). The anti-inflammatory activity of citrus peel extract highly correlated with the content of nobiletin and tangeretin (Ho and Lin, 2008). A study demonstrated that onion and potato peels also can serve as a natural source of anti-inflammatory substances (Albishi et al., 2013b).

Tea phenolic compounds have been reported to exhibit a very broad spectrum of medicinal activities. Zandi and Gordon (1999) showed that the extracts from old tea leaves (OTL) have potential as natural antioxidants. Farhoosh, et al. (2007) proved that black tea waste extracts had antioxidant activities comparable with or even better than those of old tea leave extracts. It has been demonstrated that flavonoids compounds in tea have very strong antioxidant and free radical scavenging activities, and are much more effective than vitamins $\mathrm{C}$ and $\mathrm{E}$ at protecting cells from free radical damage (Vinson et al., 1995; Wiseman et al., 1997). Green tea phenolic components inhibit intestinal uptake of glucose through rabbit intestinal epithelial cells and thus may contribute to the reduction of blood glucose levels (Kobayashi et al., 2000). Epigallocatechingallate, a phenolic component of green tea, has been found to reduce the incidence of chemically induced tumours in the oesophagus, liver, lungs, skin and stomach of experimental animals (Huang et al., 1992; Shahidi and Naczk, 2004). Epigallocatechingallate was also reported to possess effective antioxidant properties and can provide protection in vitro against both peroxyl radical and hydroxyl radical-induced oxidation of DNA (Hu and Kitts, 2001). This radical scavenging activity suggests that phenolic compounds may provide protection against carcinogens. Yen and Chen (1994) showed that aqueous extracts of black, green, oolong and pouchong tea inhibit $>90 \%$ of the mutagenicity of a selected number of chemicals toward S. typhimurium TA98 and TA100. Numerous papers have reported effects on coronary heart disease (Au Kono et al., 1996; Hertog et al., 1993a; Tijburg et al., 1997) and inhibition of carcinogenesis (Dreostic et al., 1997; Jankun et al., 1997; Majima et al., 1998; Naasani et al., 1998; Stoner and Mukhtar, 1995; Wang et al., 1991; Wiseman et al., 1997; Yang, 1997) in experimental animals by tea or tea catechins. These inhibitions were observed at all three levels of cancer progression, namely initiation, promotion, and transformation (Mitscher et al., 1997). This raises the possibility that tea drinking may reduce the incidence of both cancer and heart disease in humans. Residents of Shizuoka, Japan, where green tea is produced and consumed, were shown to have lower mortality rates from stomach, lung and liver cancers than comparable populations in non-green tea consuming area (Oguni et al., 1992). In addition they prevent teeth from decaying by inhibiting the biological activities of the cariogenic streptococci, Streptococcus mutans and Streptococcus sobrinus (Cao, 1995; Ishigami, 1991; Sakanaka, 1991, 1995). Various in vitro studies have reported that flavonols inhibit many pathogenic microorganisms including Vibrio cholerae (Boris, 1996), Streptococcus mutans (Sakanaka et al., 1992) and Shigella (Vijaya et al., 1995).

Mammalian lignans are recognized as phytoestrogens due to 
their estrogen moderating activity (Ososki and Kennelly, 2003). There is evidence that lignans from flaxseed can reduce mammary tumor size and number in rats (Thompson et al., 1996a, 1996b). Hibasami et al. (1996) reported that catechin-rich persimmon extract induced programmed cell death (apoptosis) in human lymphoid leukemia cells. Citrus flavonoids, in particular, flavanones have been shown to display anticarcinogenic and antiallergenic (Noguchi et al., 1999) activity in vitro. Wang et al. (2005) reported that lingonberries contained potent free radical scavenging activities for DPPH, peroxyl, hydroxyl and superoxide radicals. Extracts of the lingonberries also applied to cell cultures induced cancer cell apoptosis and suppressed cell damage from UV radiation. The authors suggested that the antioxidant compounds in lingonberry may make them possible candidates for a role in cancer chemoprevention and treatment.

Caffeic acid is known to selectively block the biosynthesis of leukotrienes, components involved in immunoregulation diseases, asthma and allergic reactions (Koshihara et al., 1984). Other studies have suggested that caffeic acid and some of its esters possess antitumour activity against colon carcinogenesis (Rao et al., 1993; Olthof et al., 2001). Caffeic acid derivatives have also been shown to be potent and selective inhibitors of human immunodeficiency virus type 1 (HIV-1) integrase which catalyzes the integration of the viral DNA into the host cell. Caffeic and ferulic acid derivatives have also been suggested to be potential protective agents against photooxidative skin damage (Saija et al., 1999).

Quercetin provides many health promoting benefits, including improvement of cardiovascular health, eye diseases, allergic disorders, arthritis, reducing risk for cancers and many more chronic health problems (Lakhanpal and Rai, 2007). Kerry and Abbey (1997) showed that quercetin can inhibit LDL oxidation in vitro. This action protects against atherosclerosis. Quercetin has been reported to be a potent inhibitor of human immunodeficiency virus (HIV)-1 protease (Xu et al., 2000). This compound has also been shown to decrease the infectivity of herpes simplex virus type I, poliovirus type I and parainfluenza virus in vitro (Kaul et al., 1985). Phenolic extracts from by-products (peel) of onion and potato has been reported to exhibit a potent in inhibition of LDL cholesterol oxidation, DNA scission and cyclooxygenase-2 expression (Albishi et al., 2013b).

Numerous studies on the antioxidant activity have shown that pomegranate juice contains high levels of antioxidants - higher than most other fruit juices and beverages (Gil et al., 2000; Seeram et al., 2008). The major contribution to the total antioxidant capacity of pomegranate juice is attributed to punicalagin originating from the peels (Gil et al., 2000; Tzulker et al., 2007). Clinical research studies suggest that pomegranate juice changes the blood parameters such as LDL, HDL, and cholesterol (Aviram and Dornfeld, 2001; Aviram et al., 2000, 2004; Kaplan et al., 2001), slow the rate of prostate cancer (Pantuck et al., 2006), and may be helpful against heart disease (Sumner et al., 2005), alzheimer's disease (Singh et al., 2008), cancer (Khan et al., 2007; Malik and Mukhtar, 2006; Seeram et al., 2007) improvement of sperm quality (Türk et al., 2008) and erectile dysfunction in male patients (Forest et al., 2007).

Pomegranate leaf tannins containing abundant ellagitannins, mainly ellagic acid (Lan et al. (2009), which had a potent white fat depot-lowering action in obese mice induced by a high-fat diet, and also decreased the cellular levels of triglycerides (TG) by a single orally administered lipid emulsion (Lei et al., 2007). Meng et al. (2005) observed that ellagic acid has a noticeable activity of modulating lipid metabolite of high-lipid rat and inhibiting HMG$2 \mathrm{CoA}$ reductase in vitro.

The protective role of a cashew tree leaf extract against streptozotocin-induced diabetes in both mice and rat is reported (Kamtchouing et al., 1998; Swanston-Flatt et al., 1989). The as- tringent cashew tree bark is rich in tannins and popularly used for healing wounds. It's also indicated for combating hypertension, in the treatment of gastric disturbances, and for anti-inflammatory and bactericidal treatment (Alasalvar and Shahidi, 2009).

The mango peel extract shows protective effect on hydrogen peroxide induced hemolysis, lipid peroxidation, degradation of membrane proteins and its morphological changes. (Ajila and Prasada Rao, 2007). Aqueous stem bark extract from selected species of mango, which was used in pharmaceutical formulations and used as a food supplement in Cuba under the brand name of Vimang, has been reported to display a potent in vitro and in vivo antioxidant activity and anti-inflammatory activity, and also used to prevent age-associated oxidative stress (Garrido et al., 2004; Pardo-Andreu et al., 2006; Martinez et al., 2000; Rodriguez et al., 2006; Sanchez et al., 2000).

Hydroxytyrosol in olive mill waste water (OMWW) is considered as natural antioxidants with considerable commercial and economic interest. It has high added value, due to its antioxidant and potentially beneficial (to human health) properties. Results of in vitro research demonstrate that hydroxytyrosol inhibits human LDL oxidation, scavenges free radicals, inhibits platelet aggregation, the production of nitric oxide and prostaglandin E2 for RAW264.7 mouse macrophages and the production of leucotriene for human neutrophils, and confers cell protection (Plastina et al., 2019). It also acts against both gram (+) and gram (-) bacteria. In addition, phenolic substances are the major contributors to OMWW's antimicrobial properties (Granados-Principal et al., 2010).

\section{Future considerations}

The commercial development of natural antioxidants such as polyphenols from by-products of food industry for health promoting purposes is of major interest. Before final recommendations are made it is necessary to further confirm the lack of toxicity from extracts of such by-products and to investigate further bioavailability and dosage. In addition to these considerations, more attention is needed in the following areas to add value the by-products as nutraceuticals.

Role of individual phenolic compounds;

Optimum processing conditions for by-products and their economic parameters;

Extraction parameters for high and non-toxic recoveries of phenolics;

Mode of action of different phenolic compounds in specific body organs;

The synergistic action of variety of phenolic antioxidants;

Bioavailability and bioefficacy studies in human.

Further, the bioactive components in ethnic foods and their byproducts have not been studied extensively. Therefore, more work on these areas would be helpful for the better understanding of diversified role of phenolic compounds.

\section{Conclusions}

Agro-industrial by-products serve as valuable sources of phenolic compounds such as phenolic acids, flavonoids, tannins, lignans, stilbenes, coumarins, and other nutraceuticals including carotenoids and betalains. Substantial evidence suggests that phenolic compounds and other nutraceuticals in by-products may provide one or more health beneficial effects. Phenolic compounds of varying structures possibly act as antioxidants, free radical scavengers, 
anticarcinogens, cadiopreventatives, antimicrobials, antivirals and may play a significant role in maintaining the health of humans. Therefore, importance of nutraceuticals and other natural health products has been well recognized in connection with health promotion and disease risk reduction. Growth in the use of nutraceuticals from by-products is expected to continue.

\section{References}

Abou-Gharbia, H.A., Shahidi, F., Shehata, A.A.Y., and Youssef, M.M. (1997). Effects of processing on oxidative stability of sesame oil extracted from intact and dehulled seeds. J. Am. Oil Chem. Soc. 74: 215-221.

Abou-Gharbia, H.A., Shehata, A.A.Y., and Shahidi, F. (2000). Effect of processing on oxidative stability and lipid classes of sesame oil. Food Res. Int. 33: 331-340.

Adam, A., Crespy, V., Levrat-Verny, M.A., Leenhardt, F., Leuillet, M., Demigne, C., and Remesy, C. (2002). The bioavailability of ferulic acid is governed primarily by the food matrix rather than its metabolism in intestine and liver in rats. J. Nutr. 132: 1962-1968.

Adlercreutz, C.H.T., Goldin, B.R., Gorbach, S.L., Hockerstedt, K.A.V., Watanabe, S., Hamalainen, E.K., Markkanen, M.H., Makela, T.H., Wahala, K.T., Hase, T.A., and Fotsis, T. (1995). Soybean phytoestrogen intake and cancer risk. J. Nutr. 125: 757-770.

Aguilar-Garcia, C., Gavino, G., Baragaño-Mosqueda, M., Hevia, P., and Gavino, V. (2007). Correlation of tocopherol, tocotrienol, $\gamma$-oryzano and total polyphenol content in rice bran with different antioxidant capacity assays. Food Chem. 102: 1228-1232.

Ajila, C.M., and Prasada Rao, U.J.S. (2008). Protection against hydrogen peroxide induced oxidative damage in rat erythrocytes by Mangifera indica L. peel extract. Food Chem. Toxicol. 46: 303-309.

Ajila, C.M., Bhata, S.G., and Prasada Rao, U.J.S. (2007). Valuable components of raw and ripe peels from two Indian mango varieties. Food Chem. 102: 1006-1011.

Alasalvar, C., and Bolling, B.W. (2015). Review of nut phytochemicals, fatsoluble bioactives, antioxidant components and health effects. Br. J. Nutr. 113(S2): S68-S78.

Alasalvar, C., Karamać, M., Amarowicz, R., and Shahidi, F. (2006). Antioxidant and antiradical activities in extracts of hazelnut kernel and hazelnut green leafy cover. J. Agric. Food Chem. 54: 4826-4832.

Alasalvar, C., and Shahidi, F. (2009). Tree nuts: Composition, Phytochemicals, and Health effects: An Overview. Tree nuts: Composition, Phytochemicals, and Health effects. CRC press, pp. 1-36.

Albishi, T., John, J.A., Al-Khalifa, A.S., and Shahidi, F. (2013a). Phenolic content and antioxidant activities of selected potato varieties and their processing by-products. J. Funct. Foods 5: 590-600.

Albishi, T., John, J.A., Al-Khalifa, A.S., and Shahidi, F. (2013b). Antioxidant, anti-inflammatory and DNA scission inhibitory activities of phenolic compounds in selected onion and potato varieties. J. Funct. Foods 5: 930-939.

Alongi, M., Melchior, S., and Anese, M. (2019). Reducing the glycemic index of short dough biscuits by using apple pomace as a functional ingredient. LWT-Food Sci. Tech. 100: 300-305.

Alonso, A.M., Guillén, D.A., Barroso, C.G., Puertas, B., and García, A. (2002). Determination of antioxidant activity of wine by products and its correlation with polyphenolic content. J. Agric. Food Chem. 50: 5832-5836.

Al-Weshahy, A., El-Nokety, M., Bakhete, M., and Rao, V. (2013). Effect of storage on antioxidant activity of freeze-dried potato peels. Food Res. Int. 50: 507-512.

Amakura, Y., Okada, M., Tsuji, S., and Tonogai, Y. (2000). High performance liquid chromatographic determination with photodiode array detection of ellagic acid in fresh and processed fruits. J. Chromatogr. 896: 87-93.

Amaral, J.S., Ferreres, F., Andrade, P.B., Valentão, P., Pinheiro, C., Santos, A., and Seabra, R. (2005). Phenolic profile of hazelnut (Corylus avella$n a$ L.) leaves cultivars grown in Portugal. Nat. Prod. Res. 19: 157-163.

Amarowicz, R., Naczk, M., and Shahidi, F. (2000a). Antioxidant Activity of Various Fractions Fractions of Non-tannin Phenolics of Canola Hulls. J. Agric. Food Chem. 48: 2755-2759.
Amarowicz, R., Naczk, M., and Shahidi, F. (2000b). Antioxidant activity of crude tannins of canola and rapeseed hulls. J. Am. Oil Chem. Soc. 77: 957-961.

Amarowicz, R., Naczk, M., Zadernowski, R., and Shahidi, F. (2000c). Antioxidant activity of condensed tannins of beach pea, canola hulls, evening primrose, and faba bean. J. Food Lipids 7: 195-205.

Amarowicz, R., Troszynska, A., and Shahidi, F. (2005). Antioxidant activity of almond seed extract and its fractions. J. Food Lipids. 12: 344-358.

Ambigaipalan, P., de Camargo, A.C., and Shahidi, F. (2016). Phenolic compounds of pomegranate byproducts (outer skin, mesocarp, divider membrane) and their antioxidant activities. J. Agric. Food Chem. 64 6584-6604

Ambigaipalan, P., de Camargo, A.C., and Shahidi, F. (2017). Identification of phenolic antioxidants and bioactives of pomegranate seeds following juice extraction using HPLC-DAD-ESI-MS ${ }^{n}$. Food Chem. 221: 1883-1894.

Ameer, B., Weintraub, R.A., Johnson, J.V., Yost, R.A., and Rouseff, R.L. (1996). Flavanone absorption after naringin, hesperidin, and citrus administration. Clin. Pharmac. Ther. 60: 34-40.

Anastasiadi, M., Chorianopoulos, N.G., George-John, E.N., and Serkos, A. (2009). Haroutounian antilisterial activities of polyphenol-rich extracts of grapes and vinification byproducts. J. Agric. Food Chem. 57: 457-463.

Anderson, K.J., Teuber, S.S., Gobeille, A., Cremin, P., Waterhouse, A.L., and Steinberg, F.M. (2001). Walnut polyphenolics inhibit in vitro human plasma and LDL oxidation. J. Nutr. 131: 2837-2842.

Andrade, P.B., Carvalho, A.R.F., Seabra, R.M., and Ferreira, M.A. (1998). A previous study of phenolic profiles of quince, pear, and apple purees by HPLC diode array detection for the evaluation of quince puree genuineness. J. Agric. Food Chem. 46: 968-972.

Andreasen, M.F., Christensen, L.P., Meyer, A.S., and Hansen, A. (2000). Content of phenolic acids and ferulic acid dehydrodimers in 17 rye (Secale cereale L.) varieties. J. Agric. Food Chem. 48: 2837-2842.

(2017). Annual Report of Almond Board of Califonials Almond Almanachttps://www.almonds.com/sites/default/files/2017\%20Almanac\%20 Final\%20-\%20updated\%207.5.18.pdf.

Anson, N.M., Berg, R., Havenaar, R., Bast, A., and Haenen, G.R.M.M (2009). Bioavailability of ferulic acid is determined by its bioaccessibility. J. Cereal Sci. 49: 296-300.

Aparicio-Fernández, X., Yousef, G.G., Loarca-Piña, G., De Mejia, E., and Lila M.A. (2005). Characterization of polyphenolics in the seed coat of black Jamapa bean (Phaseolus vulgaris L.). J. Agric. Food. Chem. 53 4615-4622.

Arabbi, P.R., Genovese, M.I., and Lajolo, F.M. (2004). Flavonoids in vegetable foods commonly consumed in Brazil and estimated ingestion by the Brazilian population. J. Agric. Food Chem. 52: 1124-1131.

Aramendia, M.A., García, I.M., Lafont, F., Lizaso, J., Marinas, J.M., and Urbano, F.J. (2000). Rapid determination of chlorogenic acid and related compounds in sunflower seeds by high performance liquid chromatography/atmospheric pressure chemical ionization mass spectrometry. Rapid Comm. Mass Spectrom. 14: 1019-1022.

Arcan, I., and Yemenicioğlu, A. (2009). Antioxidant activity and phenolic content of fresh and dry nuts with or without the seed coat. J. Food Comp. Anal. 22: 184-188.

Arogba, S.S. (2000). Mango (Mangifera indica) kernel: chromatographic analysis of the tannin, and stability study of the associated polyphenol oxidase activity. J. Food Comp. Anal. 13: 149-156.

Asamarai, A.M., Addis, P.B., Epley, R.J., and Krick, T.P. (1996). Wild rice hull antioxidants. J. Agric. Food Chem. 44: 126-130.

Astadi, I.R., Astuti, M., Santoso, U., and Nugraheni, P.S. (2009). In vitro antioxidant activity of anthocyanins of black soybean seed coat in human low density lipoprotein (LDL). Food Chem. 112: 659-663.

Au Kono, S., Shinchi, K., Wakabayashi, K., Honjo, S., Todoroki, I., Sakura, Y., Imanishi, K., Nishikawa, H., Ogawa, S., and Katsurada, M. (1996). Relation of green tea consumption to serum lipids and lipoproteins in Japanese men. J. Epidemiol. 6: 128-133.

Aubert, S., and Foury, C. (1981). Couleur et pigmentation antohicyanique de l'artichaut (Cynara scolymus L.). Studi sul Carciofo. In: Marzi, V., and Lattanzio, V. (Ed.). Laterza, Bari, Italy, pp. 57-76.

Aura, A.-M., Martin-Lopez, P., O'Leary, K.A., Williamson, G., Oksman-Caldentey, K.-M., Poutanen, K., and Santos-Buelga, C. (2005). In vitro 
metabolism of anthocyanins by human gut microflora. Eur. J. Nutr. 44(3): 133-142.

Avelino, A., Avelino, H.T., Roseiro, J.C., and Collaco, M.T.A. (1997). Saccharification of tomato pomace for the production of biomass. Bioresour. Technol. 61: 159-162.

Aviram, M., and Dornfeld, L. (2001). Pomegranate juice consumption inhibits serum angiotensin converting enzyme activity and reduces systolic blood pressure. Atherosclerosis 158: 195-198.

Aviram, M., Dornfeld, L., Rosenblat, M., Volkova, N., Kaplan, M., Hayek, T., Presser, D., and Fuhrman, B. (2000). Pomegranate juice consumption reduces oxidative stress, atherogenic modifications to LDL, and platelet aggregation: studies in humans and in the atherosclerotic apolipoprotein E-deficient mice. Am. J. Clin. Nutr. 71: 1062-1076.

Aviram, M., Rosenblat, M., Gaitini, D., Nitecki, S., Hoffman, A., Dornfeld, L., Volkova, N., Presser, D., Attias, J., and Liker, H. (2004). Pomegranate juice consumption for 3 years by patients with carotid artery stenosis reduces common carotid intima-media thickness, blood pressure and LDL oxidation. Clin. Nutr. 23: 423-433.

Aviram, M., Volkova, N., Coleman, R., Dreher, M., Reddy, M.K., Ferreira, D., and Rosenblat, M. (2008). Pomegranate phenolics from the peels, arils, and flowers are antiatherogenic: studies in vivo in atherosclerotic apolipoprotein e-deficient $(\mathrm{e} 0)$ mice and in vitro in cultured macrophages and lipoproteins. J. Agric. Food Chem. 56: 1148-1157.

Awad, M.A., de Jager, A., and van Westing, L.M. (2000). Flavonoid and chlorogenic acid levels in apple fruit: characterisation of variation. Scientia Horticul. 83: 249-263.

Ayed, N., Lu, H.L., and Lacroix, M. (1999). Improvement of anthocyanin yield and shelf-life extension of grape pomace by gamma irradiation. Food Res. Int. 32: 539-543.

Ayoub, M., de Camargo, A.C., and Shahidi, F. (2016). Antioxidants and bioactivities of free, esterified and insoluble-bound phenolics from berry seed meals. Food Chem. 197: 221-232.

Azar, M., Verette, E., and Brun, S. (1987). Identification of some phenolic compounds in bilberry juice Vaccinium myrtillus. J. Food Sci. 52: 1255-1257.

Azizah, A.H., Ruslawati, N.M., and Tee, S.T. (1999). Extraction and characterization of antioxidant from cocoa by-products. Food Chem. 64: 199-202.

Azuma, K., Ippoushi, K., Nakayama, M., Ito, H., Higashio, H., and Terao, J. (2000). Absorption of chlorogenic acid and caffeic acid in rats after oral administration. J. Agric Food Chem. 48: 5496-5500.

Bagchi, D., Garg, A., Krohn, R.L., Bagchi, M., Bagchi, D.J., Balmoori, J., and Stohs, S.J. (1998). Protective effects of grape seed proanthocyanidins and selected antioxidants against TPA-induced hepatic and brain lipid peroxidation and DNA fragmentation, and peritoneal macrophage activation in mice. Gen. Pharmacol. 30: 771-776.

Bai, X., Pan, R., Li, M., Li, X., and Zhang, H. (2019). HPLC profile of longan (cv. Shixia) pericarp-sourced phenolics and their antioxidant and cytotoxic effects. Molecules 24: 619.

Balasundram, N., Sundram, K., and Samman, S. (2006). Phenolic compounds in plants and agri-industrial by-products: Antioxidant activity, occurrence, and potential uses. Food Chem. 99: 191-203.

Barampama, Z., and Simard, R.E. (1993). Nutrient composition, protein quality and antinutritional factors of some varieties of dry beans (Phaseolus vulgaris L.) grown in Burundi. Food Chem. 47: 159-167.

Barberousse, H., Roiseux, O., Robert, C., Paquot, M., Deroanne, C., and Blecker, C. (2008). Review. Analytical methodologies for quantification of ferulic acid and its oligomers. J. Sci. Food Agric. 88: 14941511.

Bate-Smith, E.C., and Ribereau-Gayon, P. (1959). Leucoanthocyanins in seeds. Qual. Plant. Mater. Veg. 5: 189-198.

Bauer, J.L., Harbaum-Piayda, B., and Schwarz, K. (2012). Phenolic compounds from hydrolyzed and extracted fiber-rich by-products. LWT - Food Sci. Technol. 47: 246-254.

Baysal, T., Ersus, S., and Starmans, D.A.J. (2000). Supercritical CO2 extraction of beta-carotene and lycopene from tomato paste waste. J. Agric. Food Chem. 48: 5507-5511.

Beckman, C.H. (2000). Phenolic-storing cells: keys to programmed cell death and periderm formation in wilt disease resistance and in general defence responses in plants? Physiol. Mol. Plant Pathol. 57: 101-110.
Beecher, G.R. (2003). Overview of dietary flavonoids: Nomenclature, occurrence and intake supplement: proceedings of the third international scientific symposium on tea and human health. J. Nutr. 133: 3248S-3254S.

Beecher, G.R. (1998). Nutrient content of tomatoes and tomato products. Proceed. Soc. Exp. Biol. Med. 218: 98-100.

Ben, N.C., Ayed, N., and Metche, M. (1996). Quantitative determination of the polyphenolic content of pomegranate peel. Zeitschrift fur Lebensmittel-Untersuchung Und -Forschung 203: 374-378.

Benakmoum, A., Abbeddou, S., Ammouche, A., Kefalas, P., and Gerasopoulos, D. (2008). Valorisation of low quality edible oil with tomato peel waste. Food Chem. 110: 684-690.

Benavente-Garcia, O., Castillo, J., Marin, F.R., Ortuno, A., and Del Rio, J.A. (1997). Uses and properties of citrus flavonoids. J. Agric. Food Chem. 45: 4505-4515.

Benavente-Garcia, O., Castillo, J., Lorente, J., Ortuňo, A., and Del Rio, J.A. (2000). Antioxidant activity of phenolics extracted from Olea europe L. leaves. Food Chem. 68: 457-462.

Beninger, C.W., and Hosfield, G.L. (1998). Flavonol glycosides from the seed coat of a new manteca-type dry bean (Phaseolus vulgaris L.). J. Agric. Food Chem. 46: 2906-2910.

Bertelli, A.E., Giovannini, L., Gianness, D., Miglior, M., Bernini, W., Fregoni, M., and Bertelli, A. (1995). Antiplatelet activity of synthetic and natural resveratrol in red wine. Inter. J. Tissue Reactions 17: 1-3.

Beta, T., Nam, S., Dexter, J.E., and Sapirstein, H.D. (2005). Phenolic content and antioxidant activity of pearled wheat and roller-mill fractions. Cereal Chem. 82: 390-393.

Bianco, A., Buiarelli, F., Cartoni, G., Coccioli, F., Jasionowska, R., and Margherita, P. Analysis by liquid chromatography-tandem mass spectrometry of biophenolic compounds in olives and vegetation waters, Part I. J. Separation Sci. 26: 409-416.

Biedrzycka, E., and Amarowicz, R. (2008). Diet and health: apple polyphenols as antioxidants. Food Rev. Int. 24: 235-251.

Blaut, M., and Clavel, T. (2007). Metabolic diversity of the intestinal microbiota: implications for health and disease. J. Nutr. 137(3): 751S-755S

Bocco, A., Cuvelier, M.E., Richard, H., and Berset, C. (1998). Antioxidant activity and phenolic composition of citrus peel and seed extracts. J. Agric. Food Chem. 46: 2123-2129.

Böhm, V., Tiemeni, B., and Otto, K. (2000). Tomato marc-a good source of the efficient antioxidant lycopene? Nutritionists meet food scientist and technologists. Abstract book (p. 26). Porto, Portugal, 12-14 April 2000.

Bonilla, F., Mayen, M., Merida, J., and Medina, M. (1999). Extraction of phenolic compounds from red grape marc for use as food lipid antioxidants. Food Chem. 66: 209-215.

Bonnin, E., Saulniera, L., Brunela, M., Marota, C, Lesage-Meessenb, L., Astherb, M., and Thibault, J.F. (2002). Release of ferulic acid from agroindustrial by-products by the cell wall-degrading enzymes produced by Aspergillus niger I-1472. Enzym. Microb. Tech. 31: 1000-1005.

Booth, A.N., Emerson, O.H., Jones, F.T., and DeEds, F. (1957). Urinary metabolites of caffeic and chlorogenic acids. J. Biol. Chem. 229: 51-59.

Borie, B., Jeandet, P., Parize, A., Bessis, R., and Adrian, M. (2004). Resveratrol and stilbene synthase mRNA production in grapevine leaves treated with biotic and abiotic phytoalexin elicitors. Am. J. Enol. Vitic. 55: 60-64.

Boris, R.P. (1996). Natural products research: perspectives from a major pharmaceutical company. J. Ethnopharmacology 51: 29-38.

Bourne, L.C., and Rice-Evans, C.A. (1998). Urinary detection of hydroxycinnamates and flavonoids in humans after high dietary intake of fruit. Free Radic. Res. 28: 429-438.

Bourne, L., Paganga, G., Baxter, D., Hughes, P., and Rice-Evans, C. (2000). Absorption of ferulic acid from low-alcohol beer. Free Radical Res. 32: $273-280$

Bove, K., Lincoln, D.W., and Tsan, M.F. (2002). Effect of resveratrol on growth of 4T1 breast cancer cells in vitro and in vivo. Biochem. Biophys. Res. Comm. 291: 1001-1005.

Boyer, J., and Liu, R.H. (2004). Apple phytochemicals and their health benefits. Nutr. J. 3: 5-19.

Braddock, R.J. (1995). By-products of citrus fruit. Food Technol. 49: 74-77.

Braune, A., and Blaut, M. (2016). Bacterial species involved in the conversion of dietary flavonoids in the human gut. Gut Microbes. 7(3): 
216-234.

Bravo, L. (1998). Polyphenols: Chemistry, dietary sources, metabolism and nutritional significance. Nutr. Rev. 56: 317-333.

Brieskon, C.H., and Betz, R. (1998). Procyanidin polymers crucial to the structure of the almond seed coat. Z. Lebensm-Unters-Forsch. 187: 347-353.

Budowski, P. (1950). Sesame oil. III. Antioxidant properties of sesamol. J. Am. Oil Chem. Soc. 27: 264-267.

Bunzel, M., Ralph, J., Marita, J.M., Hatfield, R.D., and Steinhart, H. (2005) Structural elucidation of new ferulic acid-containing phenolic dimers and trimers isolated from maize bran. Tetrahedron Letters 46: 58455850.

Buranov, A.U., and Mazza, G. (2009). Extraction and purification of ferulic acid from flax shives, wheat and corn bran by alkaline hydrolysis and pressurized solvents. Food Chem. 115: 1542-1548.

Buranov, A.U., and Mazza, G. (2008). Lignin in straw of herbaceous crops: A Review. Ind. Crops Prod. 28: 237-259.

Burda, S., Oleszek, W., and Lee, C.Y. (1990). Phenolic compounds and their changes in apples during maturation and cold storage. J. Agric. Food Chem. 38: 945-948.

Caimari, A., Del Bas, J.M., Crescenti, A., and Arola, L. (2013). Low doses of grape seed procyanidins reduce adiposity and improve the plasma lipid profile in hamsters. Int. J. Obes. 37(4): 576

Çam, M., Hışıl, Y., and Durmaz, G. (2009). Classification of eight pomegranate juices based on antioxidant capacity measured by four methods. Food Chem. 112: 721-726.

Campone, L., Celano, R., Piccinelli, A.L., Pagano, I., Carabetta, S., Sanzo, R.D., Russo, M., Ibañez, E., Cifuentes, A., and Rastrelli, L. (2018). Response surface methodology to optimize supercritical carbon dioxide/cosolvent extraction of brown onion skin by-product as source of nutraceutical compounds. Food Chem. 269: 495-502.

Camussi, G., Albano, E., Tetta, C., and Bussolino, F. (1991). The molecular action of tumor necrosis factor-alpha. Eur. J. Biochem. 202: 3-14.

Cao, G. (1995). Influence of tea catechins on the synthesis of extracellular glucan and the adherence of streptococcus mutans bacteria. J. Tea Sci. 15: 57-60.

Cao, G., Muccitelli, H.U., Sanchez-Moreno, C., and Prior, R.L. (2000). Anthocyanins are absorbed in glycated forms in elder woman: a pharmacokinetic study. Am. J. Clin. Nutr. 73: 920-926.

Cardador-Martínez, A., Loarca-Piña, G., and Oomah, B.D. (2002). Antioxidant activity in common beans (Phaseolus vulgaris L.). J. Agric. Food Chem. 50: 6975-6980.

Carle, R., Keller, P., Schieber, A., Rentschler, C., Katzschner, T., Rauch, D., Fox, G.F., and Endress, H.U. (2001). Method for obtaining useful materials from the by-products of fruit and vegetable processing. Patent application WO 01/78859 A1.

Carson, K.J., Collins, J.L., and Penfield, M.P. (1994). Unrefined, dried apple pomace as a potential food ingredient. J. Food Sci. 59: 1213-1215.

Caruso, D., Visioli, F., Patelli, R., Galli, C., and Galli, G. (2001). Urinary excretion of olive oil phenols and their metabolites in humans. Metab. Clin. Exp. 50: 1426-1428.

Casa, R., Annibale, A.D., Pieruccetti, F., Stazi, S.R., Giovannozzi, S.G., and Lo Cascio, B. (2003). Reduction of the phenolic components in olive-mil wastewater by enzymatic treatment and its impact on durum wheat (Triticum durum Desf.) germinability. Chemosphere 50: 959-966.

Celotti, E., Ferrarini, R., Zironi, R., and Conte, L.S. (1996). Resveratrol content of some wines obtained from dried Valpolicella grapes: Recioto and Amarone. J. Chromatogr. A. 730: 47-52.

Cerda, B., Espin, J.C., Parra, S., Martinez, P., and Tomas-Barberan, F.A. (2004). The potent in vitro antioxidant ellagitannins from pomegranate juice are metabolised into bioavailable but poor antioxidant hydroxy-6H-dibenzopyran-6-one derivatives by the colonic microflora of healthy humans. Eur. J. Nutr. 43: 205-220.

Cerda, B., Periago, P., Espin, J.C., and Tomas-Barberan, F.A. (2005a). Identification of urolithin $A$ as a metabolite produced by human colon microflora from ellagic acid and related compounds. J. Agric. Food Chem. 53: 5571-5576.

Cerda, B., Tomas-Barberan, F.A., and Espin, J.C. (2005b). Metabolism of antioxidant and chemopreventive ellagitannins from strawberries, raspberries, walnuts, and oak-aged wine in humans: identification of biomarkers and individual variability. J. Agric. Food Chem. 53
227-235

Ćetković, G., Čanadanović-Brunet, J., Djilas, S., Savatović, S., Mandić, A., and Tumbas, V. (2008). Assessment of polyphenolic content and in vitro antiradical characteristics of apple pomace. Food Chem. 109: 340-347.

Chandrasekara, A., and Shahidi, F. (2011). Bioactivities and Antiradical Properties of Millet Grains and Hulls. J. Agri. Food Chem. 59: 95639571

Chang, L.W., Yen, W.J., Huang, S.C., and Duh, P.D. (2002). Antioxidant activity of sesame coat. Food Chem. 78: 347-354.

Chang, S.K., Alasalvar, C., Bolling, B.W., and Shahidi, F. (2016). Nuts and their co-products: The impact of processing (roasting) on phenolics, bioavailability, and health benefits-A comprehensive review. J. Funct. Foods 26: 88-122.

Chantaro, P., Devahastin, S., and Chiewchan, N. (2008). Production of antioxidant high dietary fiber powder from carrot peels. LWT - Food Sci. Technol. 41: 1987-1994.

Chen, C.K., and Pace-Asciak, C.R. (1996). Vasorelaxing activity of resveratrol and quercetin in isolated rat aorta. Gen. Pharmacol. 27: 363-366.

Chen, C.Y., Milbury, P.E., Lapsley, K., and Blumberg, J.B. (2005). Flavonoids from almond skinsare bioavailable and act synergistically with vitamins $C$ and $E$ to enhance hamster and human LDL resistance to oxidation. J. Nutr. 135: 1366-1373.

Chesson, A., Provan, G.J., Russell, W.R., Scobbie, L., Richardson, A.J., and Stewart, C. (1999). Hydroxycinnamic acids in the digestive tract of livestock and humans. J. Sci. Food Agric. 79: 373-378.

Cheynier, V., and Rigaud, J. (1986). HPLC separation and characterization of flavonols in the skins of Vitis vinifera var. Cinsault. Am. J. Enol. Viticulture 37: 248-252.

Chotimarkorn, S., Benjakul, S., and Silalai, N. (2008). Antioxidant components and properties of five long-grained rice bran extracts from commercial available cultivars in Thailand. Food Chem. 111: 636-641.

Choudhary, R., Bowser, T.J., Weckler, P., Maness, N.O., and McGlynn, W. (2009). Rapid estimation of lycopene concentration in watermelon and tomato puree by fibre optic visible reflectance spectroscopy. Post. Biol. Technol. 52: 103-109.

Choudhury, R., Srai, S.K., Debnam, E., and Rice-Evans, C.A. (1999). Urinary excretion of hydroxycinnamates and flavonoids after oral and intravenous administration. Free Radic. Biol. Med. 27: 278-286.

Clifford, M. (2001). A nomenclature for phenols with special reference to tea. Crit. Rev. Food Sci. Nutr. 41(Suppl.): 393-397.

Clifford, M.N. (1999). Chlorogenic acids and other cinnamatesnature, occurrence and dietary burden. J. Sci. Food Agric. 79: 362-372.

Clifford, M.N. (2000). Chlorogenic acids and other cinnamates - nature, occurrence, dietary burden, absorption and metabolism. J. Sci. Food Agric. 80: 1033-1042.

Clifford, M.N., Johnston, K.L., Susan, K., and Kuhnert, N. (2003). Hierarchical Scheme for LC-MSn Identification of Chlorogenic Acids. J. Agric. Food Chem. 51: 2900-2911.

Colaric, M., Veberic, R., Solar, A., Hudina, M., and Stampar, F. (2005). Phenolic acids, syringaldehyde, and juglone in fruits of different cultivars of Juglans regia L. J. Agric. Food Chem. 53: 6390-6396.

Coll, M.D., Coll, L., Laencine, J., and Tomas-Barberan, F.A. (1998). Recovery of flavanons from wastes of industrially processed lemons. Zeitschrift fur Lebensmittel-Untersuchung und-Forschung A 206: 404-407.

Cooney, J.M., Jensen, J.D., and McGhie, T.K. (2004). LC-MS identification of anthocyanins in boysenberry extract and anthocyanin metabolites in human urine following dosing. J. Sci. Food Agric. 84: 237-245.

Cornwell, D.G., and Ma, J. (2008). Nutritional benefit of olive oil: The biological effects of hydroxytyrosol and its arylating quinone adducts. J. Agric. Food Chem. 56: 8774-8786.

Cowan, M.M. (1999). Plant products as antimicrobial agents. Clin. Microbiol. Rev. 12: 564-582

Cremin, P., Kasim-Karakas, S., and Waterhouse, A.L. (2001). LC/ES-MS detection of hydroxycinnamates in human plasma and urine. J. Agric. Food Chem. 49: 1747-1750.

Crozier, A., Jaganath, I.B., and Clifford, M.N. (2009). Dietary phenolics: chemistry, bioavailability and effects on health. Nat. Prod. Rep. 26: 1001-1043.

Crozier, A., Jensen, E., Lean, M.E.J., and McDonald, M.S. (1997a). Quantitative analysis of flavonoids by reversed phase high performance liquid 
chromatography. J. Chromatogr. A 761: 315-321.

Crozier, A., Lean, M.E.J., McDonald, M.S., and Black, C. (1997b). Quantitative analysis of the flavonoid content of commercial tomatoes, onions, lettuce and celery. J. Agric. Food Chem. 43: 590-595.

Daigle, D.J., Conkerton, E.J., Sanders, T.H., and Mixon, A.C. (1988). Peanut hull flavonoids-their relationship with peanut maturity. J. Agric. Food Chem. 36: 1179-1181.

Dammak, M.I., Chakroun, I., Mzoughi, Z., Amamou, S., Mansour, H.B., Cerf, D.L., and Majdoub, H. (2018). Characterization of polysaccharides from Prunus amygdalus peels: Antioxidant and antiproliferative activities. Int. J. Biol. Macromol. 119: 198-206.

Daniel, E.M., Ratnayake, S., Kinstle, T., and Stoner, G.D. (1991). The effects of $\mathrm{pH}$ and rat intestinal contents on the liberation of ellagic acid from purified and crude ellagitannins. J. Nat. Prod. 54: 946-52.

Day, A.J., Mellon, F., Barron, D., Sarrazin, G., Morgan, M.R., and Williamson, G. (2001). Human metabolism of dietary flavonoids: Identification of plasma metabolites of quercetin. Free Radic. Res. 35: 941-952.

Day, A., Ruel, K., Neutelings, G., Crônier, D., David, H., Hawkins, S., and Chabbert, B. (2005). Lignification in the flax stem: evidence for an unusual lignin in bast fibers. Planta 222: 234-245.

de Ancos, B., Colina-Coca, C., González-Peña, D., and Sánchez-Moreno, C. (2015). Bioactive compounds from vegetable and fruit by-products. Biotechnology of Bioactive Compounds: Sources and applications. In: Gupta, V.K., and Touhy, M.G. (Ed.). John Wiley \& Sons, pp. 1-36.

de Camargo, A.C., Regitano-d'Arce, M.A.B., Biasoto, A.C.T., and Shahidi, F. (2014a). Low molecular weight phenolics of grape juice and winemaking byproducts: antioxidant activities and inhibition of oxidation of human low-density lipoprotein cholesterol and DNA strand breakage. J. Agric. Food Chem. 62: 12159-12171.

de Camargo, A.C., Regitano-d'Arce, M.A.B., Biasoto, A.C.T., and Shahidi, F. (2016). Enzyme-assisted extraction of phenolics from winemaking by-products: antioxidant potential and inhibition of alpha-glucosidase and lipase activities. Food Chem. 212: 395-402.

de Camargo, A.C., Vidal, C.M.M., Canniatti-Brazaca, S.G., and Shahidi, F. (2014b). Fortification of cookies with peanut skins: effects on the composition, polyphenols, antioxidant properties, and sensory quality. J. Agric. Food Chem. 62: 11228-11235.

de Oliveira, W.P., Biasoto, A.C.T., Marques, V.F., dos Santos, I.M., Magalhães, K., Correa, L.C., Negro-Dellacqua, M., Miranda, M.S., de Camargo, A.C., and Shahidi, F. (2017). Phenolics from winemaking by-products better decrease VLDL-cholesterol and triacylglycerol levels than those of red wine in wistar rats. J. Food Sci. 82: 2432-2437.

Déprez, S., Brezillon, C., Rabot, S., Philippe, C., Mila, I., Lapierre, C., and Scalbert, A. (2000). Polymeric proanthocyanidins are catabolized by human colonic microflora into low-molecular-weight phenolic acids. J. Nutr. 130(11): 2733-2738.

de Sousa Leite, A., Islam, M.T., Júnior, A.L.G., Sousa, J.M.D.C.E., de Alencar, M.V.O.B., Paz, M.F.C.J., Rolim, H.M.L., de Medeiros, M.D.G.F., Melo-Cavalcante, A.A.D.C., and Lopes, J.A.D. (2016). Pharmacological properties of cashew (Anacardium occidentale). African J. Biotech. 15(35): 1855-1863.

de Souza, M.Q., Teotônio, I.M.S.N., de Almeida, F.C., Heyn, G.S., Alves, P.S., Romeiro, L.A.S., and and Pratesi, C.B. (2018). Molecular evaluation of anti-inflammatory activity of phenolic lipid extracted from cashew nut shell liquid (CNSL). BMC Complement. Altern. Med. 18(1): 181.

de Vries, H.E., Ronken, E., Reinders, J.H., Buchner, B., van berkel, T.J.C., and Kuiper, J. (1998). Acute effects of oxidized low density lipoprotein on metabolic responses in macrophages. FASEB J. 12: 111-118.

de Vries, J.H.M., Hollman, P.C.H., Van Amersfoort, I., Olthof, M.R., and Katan, M.B. (2001). Red wine is a poor source of bioavailable flavonols in men. J. Nutr. 131: 745-748.

Dercks, W., and Creasy, L.L. (1989). Influence of fosetyl-Al on phytoalexin accumulation in the Plasmopara viticola-grapevine interaction. Physiol. Mol. Plant Pathol. 34: 203-213.

Díaz-Batalla, L., Widholm, J.M., Fahey, G.C. Jr, Castaño-Tostado, E., and Paredes-López, O. (2006). Chemical components with health implications in wild and cultivated Mexican common bean seeds (Phaseolus vulgaris L.). J. Agric. Food Chem. 54: 2045-2052.

Dillard, C.J., and German, J.B. (2000). Phytochemicals: nutraceuticals and human health. J. Sci. Food Agric. 80: 1744-1756.

Dimitrios, B. (2006). Sources of natural phenolic antioxidants. Trends Food
Sci. Technol. 17: 505-512.

Donnelly, L.E., Newton, R., Kennedy, G.E., Fenwick, P.S., Leung, R.H.F., Ito, K., Russell, R.E.K., and Barnes, P.J. (2004). Anti-inflammatory effects of resveratrol in lung epithelial cells: molecular mechanisms. Am. J. Physiol. Lung Cell. Mol. Physiol. 287: 774-783.

Donovan, J.L., Bell, J.R., Kasim-Karakas, S., German, J.B., Walzem, R.L., Hansen, R.J., and Waterhouse, A.L. (1999). Catechin is present as metabolites in human plasma after consumption of red wine. J. Nutr. 129: 1662-1668

Donovan, J.L., Manach, C., Faulks, R.M., and Kroon, P. (2006). Occurrence, Structure and Role in the Human Diet. Plant Secondary Metabolites. In: Crozier, A.Clifford, M.N., and Ashihara, H. (Ed.). Blackwell Publishing, Oxford, UK, pp. 303-351.

Drabińska, N., Ciska, E., Szmatowicz, B., and Krupa-Kozaka, U. (2018). Broccoli by-products improve the nutraceutical potential of gluten-free mini sponge cakes. Food Chem. 267: 170-177.

Dreher, M.L., Schantz, R.M., Holm, E.T., and Frazier, R.A. (1983). Sunflower butter: Nutritional evaluation and consumer acceptance. J. Food Sci. 48: 237.

Dreostic, I.E., Wargovich, M.J., and Yang, C.S. (1997). Inhibition of carcinogenesis by tea: The evidence from experimental studies. Crit. Rev. Food Sci. Nutr. 37: 761-770.

Duenas, M., Hernandez, T., and Estrella, I. (2006). Assessment of in-vitro antioxidant capacity of the seed coat and the cotyledon of legumes in relation to their phenolic contents. Food Chem. 98: 95-103.

Duh, P.D., Yeh, D.B., and Yen, G.C. (1992). Extraction and Identification of an Antioxidative Component from Peanut Hulls. J. Am. Oil Chem. Soc. 69: 814-818.

Dumas, Y., Dadomo, M., Di Lucca, G., and Pascal, G. (2003). Effects of environmental factors and agricultural techniques on antioxidant content of tomatoes. J. Sci. Food Agric. 83: 369-382.

Dupont, S., Mondi, Z., Willamson, G., and Price, K. (2000). Effect of variety, processing, and storage on the flavonoid glycoside and composition of lettuce and chicory. J. Agric. Food Chem. 48: 3957-3964.

Durkee, A.B. (1971). The nature of tannins in rapeseed (Brassica campestris). Phytochemistry 10: 1583-1585.

Duve, K.J., and White, P.J. (1991). Extraction and identification of antioxidants in oats. J. Am. Oil Chem. Soc. 68: 365-370.

Eberhardt, M.V., Lee, C.Y., and Liu, R.H. (2000). Nutrition: Antioxidant activity of fresh apples. Nature 405: 903-904.

Elleuch, M., Besbes, S., Roiseux, O., Blecker, C., and Attia, H. (2007). Quality characteristics of sesame seeds and by-products. Food Chem. 103: 641-650.

Erlund, I., Meririnne, E., Alfthan, G., and Aro, A. (2001). Plasma kinetics and urinary excretion of the flavanones naringenin and hesperetin in humans after ingestion of orange juice and grapefruit juice. J. Nutr. 131: $235-241$.

Esatbeyoglu, T., Juadjur, A., Wray, V., and Winterhalter, P. (2014). Semisynthetic preparation and isolation of dimeric procyanidins B1-B8 from roasted hazelnut skins (Corylus avellana L.) on a large scale using countercurrent chromatography. J. Agric. Food Chem. 62: 71017110.

Escarpa, A., and Gonzalez, M.C. (1998). High-performance liquid chromatography with diode-array detection for the determination of phenolic compounds in peel and pulp from different apple varieties. J. Chromatogr. 823: 331-337.

Escribano, J., Pedreno, M.A., Garcia-Carmona, F., and Munoz, R. (1998) Characterization of the antiradical activity ofbetalains from Beta vulgaris L. roots. Phytochem. Anal. 9: 124-127.

Espín, J.C., García-Conesa, M.T., and Tomás-Barberán, F.A. (2007). Nutraceuticals: Facts and fiction. Phytochemistry 68: 2986-3008.

Espinosa-Alonso, L.G., Lygin, A., Widholm, J.M., Valverde, M.E., and Paredes-Lopez, O. (2006). Polyphenols in wild and weedy Mexican common beans (Phaseolus vulgaris L.). J. Agric. Food Chem. 54: 44364444.

Facino, M.R., Carini, M., Aldini, G., Berti, F., Rossoni, G., Bombardelli, E., and Morazzoni, P. (1999). Diet enhanced with procyanidins enhances antioxidant activity and reduces myocardial post-ischaemic damage in rats. Life Science 64: 627-662.

FAO Products Year Report. (2004). Food and Agriculture Organization, Rome. 
FAOSTAT. (2007). FAO Statistical Database, http://www.fao.org.

FAOSTAT. (2010). http://www.fao.org/3/al375e/al375e.pdf.

FAOSTAT. (2014). http://www.fao.org/faostat/en/\#data/QD.

Farah, A., Monteiro, M., Donangelo, C.M., and Lafay, S. (2008). chlorogenic acids from green coffee extract are highly bioavailable in humans. J. Nutr. 138: 2309-2315.

Farahpour, M.R., Mirzakhani, N., Doostmohammadi, J., and Ebrahimzadeh, M. (2015). Hydroethanolic Pistacia atlantica hulls extract improved wound healing process: Evidence for mast cells infiltration, angiogenesis and RNA stability. Int. J. Surg. 17: 88-98.

Farhoosh, R., Golmovahhed, G.A., and Khodaparast, M.H.H. (2007). Antioxidant activity of various extracts of old tea leaves and black tea wastes (Camellia sinensis L.). Food Chem. 100: 231-236.

Faustino, M., Veiga, M., Sousa, P., Costa, E.M., Silva, S., and Pintado, M. (2019). Review: Agro-food byproducts as a new source of natural food additives. Molecules 24: 1056

Feki, M., Allouche, N., Bouaziz, M., Gargoubi, A., and Sayadi, S. (2006). Effect of storage of olive mill wastewaters on hydroxytyrosol concentration. Eur. J. Lipid Sci.Technol. 108: 1021-1027.

Felgines, C., Texier, O., Besson, C., Fraisse, D., Lamaison, J.-L., and Rémésy, C. (2002). Blackberry anthocyanins are slightly bioavailable in rats. J. Nutr. 132: 1249-1253.

Feng, S., and Wang, Y. (2018). Citrus phytochemicals and their potential effects on the prevention and treatment of obesity: review and progress of the past 10 years. J. Food Bioact. 4: 99-106.

Ferguson, L.R. (2001). Role of plant polyphenols in genomic stability. Mutation Res. 475: 89-111.

Fernandez de Simon, B., Perez-Ilzarbe, J., Hernandez, T., Gomez-Cordoves, C., and Estrella, I. (1992). Importance of phenolic compounds for the characterization of fruit juices. J. Agric. Food. Chem. 40: 1531-1535.

Figuerola, F., Hurtado, M.L., Estevez, A.M., Chiffelle, I., and Asenjo, F. (2005). Fibre concentrates from apple pomace and citrus peel as potential source for food enrichment. Food Chem. 91: 395-401.

Fischer, U.A., Carle, R., and Kammerer, D.R. (2011). Identification and quantification of phenolic compounds from pomegranate (Punica granatum L.) peel, mesocarp, aril and differently produced juices by HPLC-DAD-ESI/MS(n). Food Chem. 127: 807-821.

Fitzpatrick, D.F., Bing, B., and Rohdewald, P. (1998). Enothelium-dependent vascular effects of Pycnogenol. J. Cardio. Pharmacol. 32: 509515.

Foo, L.Y., and Lu, Y. (1999). Isolation and identification of procyanidins in apple pomace. Food Chem. 64: 511-518.

Foo, L.Y., Lu, Y., and Wong, H. (1998). Biphenyl-linked biflavonoids from grape pomace. Phytochemistry 47: 1137-1140.

Forest, C.P., Padma-Nathan, H., and Liker, H.R. (2007). Efficacy and safety of pomegranate juice on improvement of erectile dysfunction in male patients with mild to moderate erectile dysfunction: a randomized, placebo-controlled, double-blind, crossover study. Int. J. Impot. Res. 19: 564-567.

Fraga, C.G., Galleano, M., Verstraeten, S.V., and Oteiza, P.I. (2010). Basic biochemical mechanisms behind the health benefits of polyphenols. Mol. Aspects. Med. 31: 435-445.

Fragopoulou, E., Nomikos, T., Karantonis, H.C., Apostolakis, C., Pliakis, E., Samiotaki, M., Panayotou, G., and Antonopoulou, S. (2007). Biological activity of acetylated phenolic compounds. J. Agric. Food Chem. 55: 80-89.

Frankel, E.N., Kanner, J., German, J.B., Parks, E., and Kinsella, J.E. (1993). Inhibition of oxidation of human low-density lipoprotein by phenolic substances in red wine. Lancet 341: 454-457.

Frankel, E.N., Waterhouse, A.L., and Teissedre, P.L. (1995). Principal phenolic phytochemicals in selected California wines and their antioxidant activity in inhibiting oxidation of human low-density lipoproteins. J. Agric. Food Chem. 43: 890-894.

Friedman, M. (1997). Chemistry, biochemistry, and dietary role of potato polyphenols. A review. J. Agric. Food Chem. 45: 1523-1540.

Frison-Norrie, S., and Sporns, P. (2002a). Identification and quantification of flavonol glycosides in almond seedcoats using MALDI-TOF MS. J. Agric. Food Chem. 50: 2782-2787.

Frison-Norrie, S., and Sporns, P. (2002b). Variation in the flavonol glycoside composition of almond seedcoats as determined by MALDI-TOF mass spectrometry. J. Agric. Food Chem. 50: 6818-6822.
Fromm, M., Bayha, S., Carle, R., and Kammerer, D.R. (2012). Characterization and quantitation of low and high molecular weight phenolic compounds in apple seeds. J. Agric. Food. Chem. 60: 1232-1242.

Fromm, M., Loos, H.M., Bayha, S., Carle, R., and Kammerer, D.R. (2013). Recovery and characterisation of coloured phenolic preparations from apple seeds. Food Chem. 136: 1277-1287.

Fry, S. (1982). Phenolic components of the primary cell wall. Biochem. J. 203: 493-504.

Fuhr, U., and Kummert, A.L. (1995). The fate of naringin in humans: A key to grapefruit juice-drug interactions? Clin. Pharmac. Ther. 58: 3653737.

Fuhrman, B., Lavy, A., and Aviram, M. (1995). Consumption of Red Wine with Meals Reduces the Susceptibility of Human Plasma and Lowdensity Lipoprotein to Lipid Peroxidation. Am. J. Clin. Nutr. 61: 549554.

Fukuda, T. (2009). Walnut polyphenols: Structures and Functions. Tree nuts: composition, phytochemicals, and health effects. In: Alasalvar, C., and Shahidi, F. (Ed.). CRC press, 305-318.

Fukuda, T., Ito, H., and Yoshida, T. (2003). Antioxidative polyphenols from walnut (Juglans regia L.). Phytochemistry 63: 795-801.

Fukuda, T., Ito, H., and Yoshida, T. (2004). Effect of the walnut polyphenol fraction on oxidative stress in type 2 diabetes mice. Biofactors 21: 251-253.

Fukuda, Y., and Namiki, M. (1988). Recent studies on sesame seed oil. J. Jpn. Soc. Food Sci. Technol. 35: 552-562.

Fukuda, Y., Nagata, T., Osawa, T., and Namiki, M. (1986). Contribution of lignan analogues to antioxidative activity of refined unroasted sesame seed oil. J. Am. Oil Chem. Soc. 63: 1027-1031.

Fukuda, Y., Osawa, T., Namiki, M., and Ozaki, T. (1985). Studies on antioxidative substances in sesame seed. Agric. Biol. Chem. 49: 301-306.

Fuleki, T., and da Silva, J.M.R. (1997). Catechin and procyanidins composition of seeds from grape cultivars grown in Ontario. J. Agric. Food Chem. 45: 1156-1160.

Furuta, S., Takahashi, M., Takahata, Y., Nishiba, Y., Oki, T., Masuda, M., Kobayashi, M., and Suda, I. (2003). Radical-scavenging activities of soybean cultivars with black seed coats. Food Sci. and Technol. Res. 9(1): 73-75

Garavand, F., Madadlou, A., and Moini, S. (2017). Determination of phenolic profile and antioxidant activity of pistachio hull using HPLC DAD-ESI-MS as affected by ultrasound and microwave. Int. J. Food Prop. 20: 19-29.

Gardana, C., Guarnieri, S., Riso, P., Simonetti, P., and Porrini, M. (2007) Flavanone plasma pharmacokinetics from blood orange juice in human subjects. Br. J. Nutr. 98: 165-172.

Garrido, G., González, D., Lemus, Y., Garcia, D., Lodeiro, L., and Quintero, G. (2004). In vivo and in vitro anti-inflammatory activity of Mangifera indica L. extract (VIMANG ${ }^{\circ}$ ). Pharmacol. Res. 50: 143-149.

Garrido, P.I., Monagas, M., Gómez-Cordovés, C., and Bartolomé, B. (2007). Extraction of antioxidants from almond processing by-products. Grasas aceites 58: 130-135.

Gaya, P., Arqués, J.L., Medina, M., Álvarez, I., and Landete, J.M. (2016). A new HPLC-PAD/HPLC-ESI-MS method for the analysis of phytoestrogens produced by bacterial metabolism. Food Anal. Methods 9(2): 537-547.

Gebhardt, R. (1997). Antioxidative and protective properties of extract from leaves of the artichoke (Cynara scolymnus L.) against hydroperoxide-induced oxidative stress in cultured rat hepatocytes. Toxicol. Appl. Pharmacol. 144: 279-286.

Gebhart, R. (1998). Inhibition of cholesterol biosynthesis in primary cultured rat hepatocytes by artichoke (Cynara scolymnus L.) extracts. J. Pharmacol. Exp. Ther. 286: 1122-1128.

Geil, P.B., and Anderson, J.W. (1994). Nutrition and health implications of dry beans: a review. J. Am. Col. Nutr. 13: 549-558.

Gény, C., Rivière, G., Bignon, J., Birlirakis, N., Guittet, E., Awang, K., Litaudon, M., Roussi, F., and Dumontet, V. (2016). Anacardic acids from Knema hookeriana as modulators of $\mathrm{BCl}-\mathrm{xL} / \mathrm{Bak}$ and $\mathrm{Mcl}-1 / \mathrm{Bid}$ interactions. J. Nat. Prod. 79(4): 838-844.

George, B., Kaur, C., Khurdiya, D.S., and Kapoor, H.C. (2004). Antioxidants in tomato (Lycopersicon esculentum) as a function of genotype. Food Chem. 84: 45-51.

Ghasemian, A., Mehrabian, S., and Majd, A. (2006). Peel extracts of two 
Iranian cultivars of pomegranate (Punica granatum) have antioxidant and antimutagenic activities. Pakistan J. Biol. Sci. 9: 1402-1405.

Gil, M.I., Tomas-Berberan, A., Hess-Pierce, B., Holcroft, D.M., and Kader, A.A. (2000). Antioxidant activity of pomegranate juice and its relationship with phenolic composition and processing. J. Agric. Food Chem. 48: 4581-4589.

Girish, T.K., Pratape, V.M., and Prasada Rao, U.J.S. (2012). Nutrient distribution, phenolic acid composition, antioxidant and alpha-glucosidase inhibitory potentials of black gram (Vigna mungo L.) and its milled by-products. Food Res. Int. 46: 370-377.

Goldberg, D.M., Hahn, S.E., and Parkes, J.G. (1995). Beyond alcohol: beverage consumption and cardiovascular mortality. Clinica Chimica Acta 237: $155-187$.

Golding, J.B., McGlasson, W.B., Wyllie, S.G., and Leach, D.N. (2001). Fate of apple peel phenolics during cool storage. J. Agric. Food Chem. 49: 2283-2289.

Goli, A.H., Barzegar, M., and Sahari, M.A. (2005). Antioxidant activity and total phenolic compounds of pistachio (Pistachia vera) hull extracts. Food Chem. 92: 521-525.

Gonthier, M.P., Verny, M.A., Besson, C., Remesy, C., and Scalbert, A. (2003). Chlorogenic acid bioavailability largely depends on its metabolism by the gut microflora in rats. J. Nutr. 133: 1853-1859.

Goodwin, T.W. (1994). The Biochemistry of the Carotenoids. Chapman and Hall, New York, 2nd ed.

Görgüç, A., Bircan, C., and Yılmaz, F.M. (2019). Sesame bran as an unexploited by-product: Effect of enzyme and ultrasoundassisted extraction on the recovery of protein and antioxidant compounds. Food Chem. 283: 637-645.

Gorinstein, S., Martin-Belloso, O., Park, Y.S., Haruenkit, R., Lojek, A., Ciz, M., Caspi, A., Libman, I., and Trakhtenberg, S. (2001). Comparison of some biochemical characteristics of different citrus fruits. Food Chem. 74: 309-315.

Goto, T., Yoshida, Y., Amano, I., and Horie, H. (1996). Chemical composition of commercially available Japanese green tea. Foods Food Ingredients. J. Jpn. 170: 46-51.

Graf, E. (1992). Antioxidant potential of ferulic acid. Free Rad. Biol. Med. 13: 435-448.

Granados-Principal, S., Quiles, J.L., Ramirez-Tortosa, C.L., Sanchez-Rovira, P., and Ramirez-Tortosa, M.C. (2010). Hydroxytyrosol: from laboratory investigations to future clinical trials. Nutr Rev. 68: 191-206.

Gu, L., Kelm, M.A., Hammerstone, J.F., Beecher, G., Holden, J., Haytowitz, D., and Prior, R.L. (2003). Screening foods containing proanthocyanidins and their structural characterization using LC-MS/MS and thiolytic degradation. J. Agric. Food Chem. 51: 7513-7521.

Guandalini, B.B.V., Rodrigues, N.P., and Marczak, L.D.F. (2019). Sequential extraction of phenolics and pectin from mango peel assisted by ultrasound. Food Res. Int. 119: 455-461.

Gulcan, O., Osman, S., Nilgun, G.B., and Zehra, K. (2004). Antibacterial activities and total phenolic contents of grape pomace extracts. J. Food Sci. Agric. 84: 1807-1811.

Gülsen, A., Makris, D.P., and Kefalas, P. (2007). Biomimetic oxidation of quercetin: isolation of a naturally occurring quercetin heterodimer and evaluation of its in vitro antioxidant properties. Food Res. Int. 40: 7-14.

Gunduc, N., and El, S.N. (2003). Assesing antioxidant activities of phenolic compounds of common Turkish food and drinks on in vitro low-density lipoprotein oxidation. J. Food Sci. 68: 2591-2595.

Guo, C., Yang, J., Wei, J., Li, Y., Xu, J., and Jiang, Y. (2003). Antioxidant activities of peel, pulp and seed fractions of common fruits as determined by FRAP assay. Nutr. Res. 23: 1719-1726.

Hakim, I.A., Alsaif, M.A., Alduwaihy, M., Al-Rubeaan, K., Al-Nuaim, A.R., and Al-Attas, O.S. (2003). Tea consumption and the prevalence of coronary heart disease in Saudi adults: results from a Saudi national study. Prev. Med. 36: 64-70.

Halliwell, B. (1996). Oxidative stress, nutrition and health. Experimental strategies for optimization of nutritional antioxidant intake in humans. Free Rad. Res. 25: 57-74.

Hamad, F., and Mubofu, E. (2015). Potential biological applications of biobased anacardic acids and their derivatives. Int. J. Mol. Sci. 16(4): 8569-8590.

Harborne, J.B. (1982). Introduction to Ecological Biochemistry, second ed.
Academic Press, New York, NY.

Harborne, J.B. (1989). General procedures and measurement of total phenolics. Methods in plant biochemistry: Volume 1 Plant Phenolics. In: Harborne, J.B. (Ed.). Academic Press, London, UK, pp. 1-28.

Harborne, J.B. (1999). Classes and functions of secondary products from plants. Chemicals from Plants-erspectives on Plant Secondary Products. In: Walton, J.N., and Brown, D.E. (Ed.). Imperial College Press, London, UK, pp. 1-25.

Harborne, J.B., and Simmonds, N.W. (1964). Biochemistry of phenolic compounds. Academic press, London, UK, p. 101.

Harborne, J.B., and Williams, C.A. (2000). Advances in flavonoid research since 1992. Phytochem. 55: 481-504.

Harborne, J.B.Baxter, H., and Moss, G.P. (Ed.). (1999). Phytochemical dictionary: Handbook of bioactive compounds from plants, 2nd ed. Taylor and Francis, London, UK.

Harder, H., Tetens, I., Let, M.B., and Meyer, A.S. (2004). Rye bran bread intake elevates urinary excretion of ferulic acid in humans, but does not affect the susceptibility of LDL to oxidation ex vivo. Eur. J.Nutr. 43: 230-236.

Harding, V.K., and Heale, J.B. (1980). Isolation and identification of the antifungal compounds accumulating in the induced resistance response of carrot root slices to Botrytis cinerea. Physiol. Plant Pathol. 17: 277-289.

He, L., Xu, H., Liu, X., He, W., Yuan, F., Hou, Z., and Gao, Y. (2011). Identification of phenolic compounds from pomegranate (Punica granatum $\mathrm{L}$.) seed residues and investigation into their antioxidant capacities by HPLC-ABTS ${ }^{+}$assay. Food Res. Int. 44: 1161-1167.

Heinecke, J.W. (1997). Mechanisms of oxidative damage of low density lipoprotein in human atherosclerosis. Curr. Opin. Lipidol. 8: 268-274.

Heleno, S.A., Martins, A., Queiroz, M.J.R., and Ferreira, I.C. (2015). Bioactivity of phenolic acids: Metabolites versus parent compounds: A review. Food Chem. 173: 501-513.

Hernández-Hernández, C., Morales-Sillero, A., Fernández-Bolaños, J., Bermúdez-Oria, A., Morales, A.A., and Rodríguez-Gutiérreza, G. (2019) Cocoa bean husk: industrial source of antioxidant phenolic extract. J. Sci. Food Agric. 99: 325-333.

Herrmann, K. (1989). Occurrence and content of hydroxycinnamic and hydroxybenzoic acid compounds in food. Crit Rev Food Sci Nutr. 28: 315-347.

Hertog, M.G.L., Feskens, E.J.M., Hollman, P.C.H., Katan, M.B., and Kromhout, D. (1993a). Dietary antioxidant flavonoids and risk of coronary heart disease. The Zutphen elderly Study. The Lancet. 342: 10071011.

Hertog, M.G.L., Hollman, P.C.H., and Katan, M.B. (1992). Content of potentially anticarcinogenic flavonoids of 28 vegetables and 9 fruits commonly consumed in the Netherlands. J. Agric. Food Chem. 40: 2379-2381.

Hertog, M.G.L., Hollman, P.C.H., and van de Putte, B. (1993b). Content of Potentially Anticarcinogenic Flavonids of Tea Infusions, Wines, and Fruit Juices. J. Agric. Food Chem. 41: 1242-1246.

Hertog, M.G.L., Sweetman, P.M., Fehily, A.M., Elwood, P.C., and Kromhout, D. (1997). Antioxidant flavonols and ischemic heart disease in a Welsh population of men: The Caerphilly Study. Am. J. Clin. Nutr. 65: 1489-1494.

Hęś, M., Górecka, D., and Dziedzic, K. (2012). Antioxidant properties of extracts from buckwheat by-products. Acta Sci. Pol., Technol. Aliment. 11(2): 167-174.

Hibasami, H., Achiwa, Y., Fujikawa, T., and Komiya, T. (1996). Induction of programmed cell death (apoptosis) in human lymphoid leukemia cells by catechin compounds. Anticancer Res. 16: 1943-1946.

Hidalgo, M., Oruna-Concha, M.J., Kolida, S., Walton, G.E., Kallithraka, S., Spencer, J.P., and de Pascual-Teresa, S. (2012). Metabolism of anthocyanins by human gut microflora and their influence on gut bacterial growth. J. Agric. Food Chem. 60(15): 3882-3890.

Ho, S.C., and Lin, C.C. (2008). Investigation of heat treating conditions for enhancing the anti-inflammatory activity of citrus fruit (Citrus reticulata) peels. J. Agric. Food Chem. 56: 7976-7982.

Hollman, P.C.H. (2004). Absorption, bioavailability, and metabolism of flavonoids. Pharm. Biol. 42: 74-83.

Hollman, P.C.H., van Trijp, J.M.P., Buysman, M.N.C.P., v.d.Gaag, M.S., Mengelers, M.J.B., de Vries, J.H.M., and Katan, M.B. (1997). Relative 
bioavailability of the antioxidant flavonoid quercetin. FEBS Lett. 418 152-156.

Hollman, P.C., Bijsman, M.N., van Gameren, Y., Cnossen, E.P., de Vries, J.H., and Katan, M.B. (1999). The sugar moiety is a major determinant of the absorption of dietary flavonoid glycosides in man. Free Radic. Res. 31: 569-573.

Horton, K.L., Morgan, J.M., Uhrin, L.E., Boyle, M.R., Altomare, L.P. Laskowsky, C., Walter, K.E., Stanton, M.M., Newman, L.M., and Capuzzi, D.M. (1999). The effect of walnut on serum lipids consumed as part of the national cholesterol educational panel step I diet. J. Am. Dietetic Assoc. 99: 109-112.

Hu, C., and Kitts, D.D. (2001). Evaluation of antioxidant activity of epigallocatechin gallate in biphasic model systems in vitro. Mol. Cell. Biochem. 218: 147-155.

Huang, M.T., Ho, C.T., and Lee, C.Y. (1992). Phenolic compounds in food and their effects on health II. ACS symposium series 507. American Chemical Society, Washington, DC.

Huang, S.C., Yen, G.C., Chang, L.W., Yen, W.J., and Duh, P.D. (2003). Identification of an antioxidant, ethyl protocatechuate, in peanut seed testa. J. Agric. Food Chem. 51: 2380-2383.

Hung, P.V. (2016). Phenolic compounds of cereals and their antioxidant capacity. Crit. Rev. Food Sci. Nutr. 56: 25-35.

Ichiyanagi, T., Shida, Y., Rahman, M.M., Hatano, Y., and Konishi, T. (2005). Extended glucuronidation is another major path of cyanidin 3-Obeta-d-glucopyranoside metabolism in rats. J. Agric. Food Chem. 53 7312-7319.

International Nut and Dried Fruit Council (INDFC). (2017). https:// www.nutfruit.org/files/tech/1524481168_INC_Statistical_Yearbook 2017-2018.pdf.

Iqbal, S., Bhanger, M.I., Akhtar, M., Anwar, F., Ahmed, K.R., and Anwer, T. (2006). Antioxidant properties of methanolic extracts from leaves of Rhazya stricta. J. Med. Food 9: 270-275.

Iqbal, S., Haleem, S., Akhtar, M., Zia-ul-Haq, M., and Akbar, J. (2008). Efficiency of pomegranate peel extracts in stabilization of sunflower oil under accelerated conditions. Food Res. Int. 41: 194-200.

Isanga, J., and Zhang, G.N. (2007). Biologically active components and nutraceuticals in peanuts and related products: Review. Food Rev. Int 23: $123-140$.

Ishigami, T. (1991). Antibacterial activity of tea polyphenols against foodborne, cariogenic and phytopathogenic bacteria. Proc. of Int. Symp. on Tea Science Shizuoka, Japan, pp. 248-252, 26-29 August 1991.

Ishii, K., Furuka, T., and Kasuya, Y. (2000). Mass spectrometric identification and high-performance liquid chromatography determination of a flavonoid glycoside naringin in human urine. J. Agric. Food Chem. 46: $56-59$.

Ishii, T. (1997). Structure and functions of feruloylated polysaccharides. Plant Sci. 127: 111-127

Ismail, A., Marjan, Z.M., and Foong, C.W. (2004). Total antioxidant activity and phenolic content in selected vegetables. Food Chem. 87 581-586.

Ito, H., Gonthier, M.P., Manach, C., Morand, C., Mennen, L., Rémésy, C., and Scalbert, A. (2005). Polyphenol levels in human urine after intake of six different polyphenol-rich beverages. Br. J. Nutr. 94: 500-509.

IUPAC. (1976). Nomenclature of cyclitols. Biochem. J. 153: 23-31.

Izumi, T., Piskula, M.K., Osawa, S., Obata, A., Tobe, K., Saito, M., Kataoka, S., Kubota, Y., and Kikuchi, M. (2000). Soy isoflavone aglycones are absorbed faster and in higher amounts than their glucosides in humans. J. Nutr. 130: 1695-1699.

Jager, U., and Nguyen-Duong, H. (1999). Relaxant Effect of Trans-resveratrol on Isolated Porcine Coronary Arteries. Arzneimittel-Forschung/ Drug Res. 49: 207-211.

Jang, M., Cai, L., Udeani, G.O., Slowing, K.V., Thomas, C.F., Beecher, C.W.W., Fong, H.H.S., Farnsworth, N.R., Kinghorn, A.D., Mehta, R.G., Moon, R.C., and Pezzuto, J.M. (1997). Cancer chemopreventive activity of resveratrol, a natural product derived from grapes. Science 275 218-220.

Jankun, J., Selman, S.H., Swiercz, R., and Skrzypczak-Jankun, E. (1997). Why drinking green tea could prevent cancer. Nature 387: 561.

Jaramillo-Carmona, S., Rodríguez-Arcos, R., Guillén-Bejarano, R., and Jiménez-Araujo, A. (2019). Hydrothermal treatments enhance the solubilityand antioxidant characteristics of dietary fiberfrom asparagus by-products. Food Bioprod. Process. 114: 175-184.

Jayaprakasha, G.K., Singh, R.P., and Sakariah, K.K. (2001). Antioxidant activity of grape seed (Vitis vinifera) extracts on peroxidation models in vitro. Food Chem. 73: 285-290.

Jeong, S.M., Kim, S.Y., Kim, D.R., Jo, S.C., Nam, K.C., Ahn, D.U., and Lee, S.C. (2004). Effect of heat treatment on the antioxidant activity of extracts from citrus peels. J. Agric. Food Chem. 52: 3389-3393.

Jham, G.N. (1996). High-performance liquid chromatographic quantitation of phloridzin in apple seed, leaf and callus. J. Chromatogr. A 719: 444-449.

Johnsson, P., Peerlkamp, N., Kamal-Eldin, A., Andersson, R.E., Andersson, R., Lundgren, L.N., and Aman, P. (2002). Polymeric fractions containing phenol glucosides in flaxseed. Food Chem. 76: 207-212.

Jian, H.Y., An, C.C., Feng, J., Ci, Y.X., Li, Y., Sugisawa, A., Izumitani, M., and Chen, Z.L. (1999). Effect of caffeic acid on the tumor cells U937 evaluated by an electrochemical voltammetric method. Chinese Chem. Lett. 10: 781-782.

Kabir, F., Tow, W.W., Hamauzu, Y., Katayama, S., Tanaka, S., and Nakamura, S. (2015). Antioxidant and cytoprotective activities of extracts prepared from fruit and vegetable wastes and by-products. Food Chem. 167: 358-362.

Kabuki, T., Nakajima, H., Arai, M., Ueda, S., Kuwabara, Y., and Dosako, S. (2000). Characterization of novel antimicrobial compounds from mango (Mangifera indica L.) kernel seeds. Food Chem. 71: 61-66.

Kähkönen, M.P., Heinämäki, J., Ollilainen, V., and Heinonen, M. (2003). Berry anthocyanins: Isolation, identification and antioxidant activities. J. Sci. Food Agric. 83: 1403-1411.

Kähkönen, M.P., Hopia, A.I., Vuorela, H.J., Rauha, J.P., Pihlaja, K., and Kujala, T.S. (1999). Antioxidant activity of plant extracts containing phenolic compounds. J. Agric. Food Chem. 47: 3954-3962.

Kallithraka, S., Garcia-Viguera, C., Bridle, P., and Bakker, J. (1995). Survey of solvents for the extraction of grape seed phenolics. Phytochem. Anal. 6: 265-267.

Kamal-Eldin, A., Pettersson, D., and Appelqvist, L.A. (1995). Sesamin (a compound from sesame oil) increases tocopherol levels in rats fed ad libitum. Lipids 30: 499-505.

Kamath, V., and Rajini, P.S. (2007). The efficacy of cashew nut (Anacardium occidentale L.) skin extract as a free radical scavenger. Food Chem. 103: 428-433.

Kamiloglu, S., Capanoglu, E., Bilen, F.D., Gonzales, G.B., Grootaert, C., de Wiele, T.V., and Van Camp, J. (2016). Bioaccessibility of polyphenols from plant-processing byproducts of black carrot (Daucus carota L.) J. Agric. Food Chem. 64: 2450-2458.

Kammerer, D., Claus, A., Carle, R., and Schieber, A. (2004). Polyphenol screening of pomace from red and white grape varieties (Vitis vinifera L.) by HPLC-DAD-MS/MS. J. Agric. Food Chem. 52: 4360-4367.

Kamtchouing, P., Sokeng, S.D., Moundipa, P.F., Watcho, P., Jatsa, H.B., and Lontsi, D. (1998). Protective role of Anacardium occidentale extract against streptozotocin-induced diabetes in rats. J. Ethnopharmacol. 62: 95-99.

Kanazawa, K., and Sakakibara, H. (2000). High content of dopamine, a strong antioxidant, in cavendish banana. J. Agric. Food Chem. 48 844-848.

Kanaze, F.I., Bounartzi, M.I., Georgarakis, M., and Niopas, I. (2007). Pharmacokinetics of the citrus flavanone aglycones hesperetin and naringenin after single oral administration in human subjects. Eur. J. Clin Nutr. 61: 472-477.

Kang, M.H., Katsuzaki, H., and Osawa, T. (1998a). Inhibition of 2,2'-azobis (2,4-dimethylvaleronitrile) induced lipid peroxidation by sesaminols. Lipids 33: 1031-1036.

Kang, M.H., Naito, M., Tsujihara, N., and Osawa, T. (1998b). Sesamolin inhibits lipid peroxidation in rat liver and kidney. J. Nutr. 128: 10181022

Kang, M.H., Yoshichika, K., Michitaka, N., and Toshihiko, O. (1999). Dietary defatted sesame flour decreases susceptibility to oxidative stress in hypercholesterolemic rabbits. J.Nutr. 129: 1885-1890.

Kanner, J., Harel, S., and Granit, R. (2001). Betalains - a new class of dietary cationized antioxidants. J. Agric. Food Chem. 49: 5178-5185.

Kano, M., Takayanagi, T., Harada, K., Sawada, S., and Ishikawa, F. (2006). Bioavailability of Isoflavones after Ingestion of Soy Beverages in Healthy Adults. J. Nutr. 136: 2291-2296. 
Kao, T.H., Chien, J.T., and Chen, B.H. (2008). Extraction yield of isoflavones from soybean cake as affected by solvent and supercritical carbon dioxide. Food Chem. 107: 1728-1736.

Kaplan, M., Hayek, T., Raz, A., Coleman, R., Dornfeld, L., Vaya, J., and Aviram, M. (2001). Pomegranate juice supplementation to atherosclerotic mice reduces macrophage lipid peroxidation, cellular cholesterol accumulation and development of atherosclerosis. J. Nutr. 131: 2082-2089.

Karakaya, S. (2004). Bioavailabilty of phenolic compounds. Crit. Rev. Food Sci. Nutr. 44: 453-464.

Kaul, T.N., Middletown, E.J., and Ogra, P.L. (1985). Antiviral effect of flavonoids. Antiviral effect of flavonoids on human viruses. J. Med. Virol. 15: 71-79.

Keefe, S.F., and Wang, H. (2006). Effect of Peanut Skin Extract on Quality and Storage Stability of Beef Products. Meat Sci. 73: 278-286.

Kern, S.M., Bennett, R.N., Mellon, F.A., Kroon, P.A., and Garcia-Conesa, M.T. (2003a). Absorption of hydroxycinnamates in humans after high-bran cereal consumption. J. Agric. Food Chem. 51: 6050-6055.

Kerry, N.L., and Abbey, M. (1997). Red wine and fractionated phenolic compounds prepared from red wine inhibits low density lipoprotein oxidation in vitro. Atherosclerosis 135: 93-102.

Khan, N., Afaq, F., Kweon, M.H., Kim, K., and Mukhtar, H. (2007). Oral consumption of pomegranate fruit extract inhibits growth and progression of primary lung tumors in mice. Cancer Res. 67: 3475-3482.

Kim, J.W., and Mazza, G. (2006). Optimization of extraction of phenolic compounds from flax shives by pressurized low-polarity water. J. Agric. Food Chem. 54: 7575-7584.

Kim, D.O., Padilla-Zakour, O.I., and Griffiths, P.D. (2004). Flavonoids and antioxidant capacity of various cabbage genotypes at juvenile stage. J. Food. Sci. 69: 685-689.

Kim, K.H., Yang, R.T.R., and Cui, S.W. (2006). Phenolic acid profiles and antioxidant activities of wheat bran extracts and the effect of hydrolysis conditions. Food Chem. 95: 466-473.

King, A., and Young, G. (1999). Characteristics and occurrence of phenolic phytochemicals. J. Am. Dietetic Association 99: 213-218.

Knockaert, D., Raes, K., Wille, C., Struijs, K., and Van Camp, J. (2012). Metabolism of ferulic acid during growth of Lactobacillus plantarum and Lactobacillus collinoides. J. Sci. Food Agric. 92(11): 2291-2296.

Kobayashi, Y., Suzuki, M., Satsu, H., Arai, S., Hara, Y., Suzuki, K., Miyamoto, Y., and Shimizu, M. (2000). Green tea polyphenols inhibit the sodiumdependent glucose transportre of intestinal epithelial cells by a competitive mechanism. J. Agric. and Food Chem. 48: 5618-5623.

Kong, K.W., Khoo, H.E., Prasad, K.N., Ismail, A., Tan, C.P., and Rajab, N.F. (2010). Revealing the power of the natural red pigment lycopene. Molecules 15: 959-987.

Konishi, Y., Hitomi, Y., and Yoshioka, E. (2004). Intestinal absorption of $p$ coumaric and gallic acids in rats after oral administration. J. Agric Food Chem. 52: 2527-2532.

Konishi, Y., Kobayashi, S., and Shimizu, M. (2003). Transepithelial transport of $p$-coumaric acid and gallic acid in caco- 2 cell monolayers. Biosci. Biotechnol. Biochem. 67: 2317-2324.

Kornsteiner, M., Wagner, K.H., and Elmadfa, I. (2006). Tocopherols and total phenolics in 10 different nut types. Food Chem. 98: 381-387.

Koshihara, Y., Neichi, T., Murota, S.I., Lao, A.N., Fujimoto, Y., and Tatsuno, T. (1984). Caffeic acid is a selective inhibitor for leukotriene biosynthesis. Biochem. Biophy. Acta 792: 92-97.

Kovac, V., Alonso, E., and Revilla, E. (1995). The effect of adding supplementary quantities of seeds during fermentation on the phenolic composition of wines. Americ. J. Enol. Viticulture 46: 363-367.

Krinsky, N.I. (1994). The biological properties of carotenoids. Pure and Appl. Chem. 66: 1003-1010.

Kris-Etherton, P.M., and Keen, C.L. (2002). Evidence that the antioxidant flavonoids in tea and cocoa are beneficial for cardiovascular health. Curr. Opin. Lipidology 13: 41-49.

Kroon, P.A., and Williamson, G. (1999). Hydroxycinnamates in plants and food: Current and future perspectives. J. Sci. Food Agric. 79: 355-361.

Krygier, K., Sosulski, F., and Hogge, L. (1982). Free, esterified and insoluble phenolic acids. 2. Composition of phenolic in rapeseed flour and hulls. J. Agric. Food Chem. 30: 334-336.

Kubo, I., Masuoka, N., Ha, T.J., and Tsujimoto, K. (2006). Antioxidant activity of anacardic acids. Food Chem. 99: 555-562.
Kubo, I., Muroi, H., Himejima, M., Yamagiwa, Y., Mera, H., Tokushima, K., Ohta, S., and Kamikawa, T. (1993a). Structure-antibacterial activity relationships of anacardic acids. J. Agric. Food Chem. 41: 1016-1019.

Kubo, I., Ochi, M., Vieira, P.C., and Komatsu, S. (1993b). Antitumor agents from the cashew (Anacardium occidentale) apple juice. J. Agric. Food Chem. 41: 1012-1015.

Kujala, T.S., Loponen, J.M., Kika, K.D., and Pihlaja, K. (2000). Phenolics and betacyanins in red beetroot (Beta vulgaris) root: distribution and effect of cold storage on the content of total phenolics and three individual compounds. J. Agric. Food Chem. 48: 5338-5342.

Kujala, T., Loponen, J., and Pihlaja, K. (2001). Betalains and phenolics in red beetroot (Beta vulgaris) peel extracts: extraction and characterisation. Zeitschrift fur Naturforschung C 56: 343-348.

Kulkarni, A.P., Aradhya, S.M., and Divakar, S. (2004). Isolation and identification of a radical scavenging antioxidant - punicalagin from pith and carpellary membrane of pomegranate fruit. Food Chem. 87: 551-557.

Kumar, A., Pundhir, V.S., and Gupta, K.C. (1991). The role of phenols in potato tuber resistance against soft rot by Erwinia carotovora ssp. carotovora. Potato Research 34: 9-16.

Kuo, S.M. (1996). Antiproliferative potency of structurally distinct dietary flavonoids on human colon cancer cells. Cancer Lett. 110: 41-48.

Labuckas, D.O., Maestri, D.M., Perelló, M., Martínez, M.L., and Lamarque, A.L. (2008). Phenolics from walnut (Juglans regia L.) kernels: Antioxidant activity and interactions with proteins. Food Chem. 107: 607-612.

Laddomada, B., Caretto, S., and Mita, G. (2015). Wheat bran phenolic acids: bioavailability and stability in whole wheat-based foods. Molecules 20: 15666-15685.

Lafay, S., and Gil-Izquierdo, A. (2008). Bioavailability of phenolic acids. Phytochem Rev. 7: 301-311.

Lafay, S., Morand, C., Manach, C., Besson, C., and Scalbert, A. (2006). Absorption and metabolism of caffeic acid and chlorogenic acid in the small intestine of rats. Br. J. Nutr. 96: 39-46.

Lafka, T., Sinanoglou, V., and Lazos, E.S. (2007). On the extraction and antioxidant activity of phenolic compounds from winery wastes. Food Chem. 104: 1206-1214.

Lakhanpal, P., and Rai, D.K. (2007). Quercetin: A versatile flavonoid. Int. J. Med. 2: 20-35.

Lakshminarayana, S., Subhadra, N.V., and Subramanyam, N. (1979). Some aspects of developmental physiology of mango fruit. J. Horticul. Sci. 45: 133-142.

Lambert, J.D., and Yang, C.S. (2003). Cancer chemopreventive activity and bioavailability of tea and tea polyphenols. Mutat. Res. 523-524: 201-208.

Lampe, J. (1999). Health effects of vegetables and fruit: Assessing mechanisms of action in human experimental studies. Am. J. Clin. Nutr. 70 475S-490S.

Lan, J., Lei, F., Hua, L., Wang, Y., Xing, D., and Du, L. (2009). Transport behavior of ellagic acid of pomegranate leaf tannins and its correlation with total cholesterol alteration in HepG2 cells. Biomed. Chromatogr 23: 531-536.

Langcake, P., and Pryce, R.J. (1976). The production of resveratrol by Vitis vinifera and other members of the Vitaceae as a response to infection or injury. Physiol. Plant Pathol. 9: 77-86.

Langcake, P., and Pryce, R.J. (1977a). The production of resveratrol and the viniferins by grapevines in response to ultraviolet irradiation. Phytochem. 16: 1193-1196.

Langcake, P., and Pryce, R.J. (1977b). A new class of phytoalexins from grapevines. Experientia 32: 151-152.

Langcake, P., Cornford, C.A., and Pryce, R.J. (1979). Identification of pterostilbene as a phytoalexin from Vitis vinifera leaves. Phytochem. 18: 1025-1027.

Larrauri, J.A., Ruperez, P., and Saura-Calixto, F. (1997a). Effect of drying temperature on the stability of polyphenols and antioxidant activity of red grape pomace peels. J. Agric. Food Chem. 45: 1390-1393.

Larrauri, J.A., Ruperez, P., and Saura-Calixto, F. (1997b). Mango peels with high antioxidant activity. Z. Lebensm-Untersuch und-Forsch. 205: 39-42.

Larrauri, J.A., Ruperez, P., Borroto, B., and Saura-Calixto, F. (1996). Mango peels as a new tropical fibre: preparation and characterization. LWT 
- Food Sci. Technol. 29: 729-733.

Larrauri, J.A., Sanchez-Moreno, C., and Saura-Calixto, F. (1998). Effect of temperature on the free radical scavenging capacity of extracts from red and white grape pomace peels. J. Agric. Food Chem. 46: 2694-2697.

Lau, W.K., Chuyen, H.V., and Vuong, Q.V. (2018). Physical properties, carotenoids and antioxidant capacity of carrot (Daucus carota L.) peel as influenced by different drying treatments. Int. J. Food Eng.

Lattanzio, V., Cardinali, A., di Venere, D., Linsalata, V., and Palmieri, S. (1994). Browing phenomena in stored artichoke (Cynara scolymus L.) heads: enzymatic or chemical reactions? Food Chem. 50: 1-7.

Lattanzio, V., and van Sumere, C.F. (1987). Changes in phenolic compounds during the development and cold storage of artichoke (Cynara scolymus L.). Food Chem. 24: 37-50.

Laurens, C., Fourneau, R., Hocquemiller, A., Cave, C., and Bories and Loiseau, P.M. (1997). Antivectorial activities of cashew nut shell extracts from Anacardium occidentale L. Phyto. Res. 11: 145-146.

Lavedrine, F., Zmirou, D., Ravel, A., Balducci, F., and Alary, J. (1999). Blood cholesterol and walnut consumption: A cross-sectional survey in France. Preventive Med. 28: 333-339.

Lazarus, S.A., Adamson, G.E., Hammerstone, J.F., and Schmitz, H.H. (1999). High performance liquid chromatography/mass spectrometry analysis of proanthocyanidins in food and beverages. J. Agric. Food Chem. 47: 3693-3701.

Lee, M.-J., Maliakal, P., Chen, L., Meng, X., Bondoc, F.Y., Prabhu, S., Lambert, G., Mohr, S., and Yang, C.S. (2002). Pharmacokinetics of tea catechins after ingestion of green tea and (-)-epigallocatechin-3-gallate by humans: Formation of different metabolites and individual variability. Cancer Epidemiol. Biomarkers Prev. 11: 1025-1032.

Lee, S., CKim, J.H., Jeong, S.M., Kim, D.R., Ha, J.U., Nam, K.C., and Ahn, D.U. (2003). Effect of far-infrared radiation on the antioxidant activity of rice hulls. J. Agric. Food Chem. 51: 4400-4403.

Lee, Y.S., and Reidenberg, M.M. (1998). A method for measuring naringenin in biological fluids and its disposition from grapefruit juice by man. Pharmacology 56: 314-317.

Lei, F., Xing, D.-M., Xiang, L., Zhao, Y.-N., Wang, W., Zhang, L.J., and Du, L.J. (2003). Pharmacokinetic study of ellagic acid in rat after oral administration of pomegranate leaf extract. J. Chromatogr. B. 796: 189-94.

Lei, F., Zhang, X.N., Wang, W., Xing, D.M., Xie, W.D., Su, H., and Du, L.J. (2007). Evidence of anti-obesity effects of the pomegranate leaf extract in high-fat diet induced obese mice. Int. J. Obesity (London) 31 1023-1029.

de Leonardis, A., Macciola, V., and di Domenico, N. (2005). A first pilot study to produce a food antioxidant from sunflower seed shells ( $\mathrm{He}$ lianthus annuus). Eur. J. Lipid Sci. 107: 220-227.

Lesage-Meesen, L., Navarro, D., Maunier, S., Sigoillot, J.C., Lorquin, J., Delattre, M., Simon, J-L., Asther, M., and Labat, M. (2001). Simple phenolic content in olive oil residues as a function of extraction systems. Food Chem. 75: 501-507.

Leung, J., Fenton, T.W., Mueller, M.M., and Clandinin, D.R. (1979). Condensed tannins of rapeseed meals. J. Food Sci. 44: 1313-1316.

Li, B.B., Smith, B., and Hossain, M.M. (2006a). Extraction of phenolics from citrus peels: I. Solvent extraction method. Sep. Pur. Technol. 48: 182-188.

Li, B.B., Smith, B., and Hossain, M.M. (2006b). Extraction of phenolics from citrus peels. II. Enzyme-assisted extraction method. Sep. Sci. Technol. 48: 189-196.

Li, F., Zhang, X., Zheng, S., Lu, K., Zhao, G., and Ming, J. (2016). The composition, antioxidant and antiproliferative capacities of phenolic compounds extracted from tartary buckwheat bran [Fagopyrum tartaricum (L.) Gaerth]. J. Funct. Foods 22: 145-155

Li, Y., Guo, C., Yang, J., Wei, J., Xu, J., and Cheng, S. (2006c). Evaluation of antioxidant properties of pomegranate peel extract in comparison with pomegranate pulp extract. Food Chem. 96: 254-260.

Liggins, J., Grimwood, R., and Bingham, S.A. (2000). Extraction and quantification of lignan phytoestrogens in food and human samples. Anal. Biochem. 287: 102-109.

Lin, L.Z., and Harnly, J.M. (2007). A screening method for the identification of glycosylated flavonoids and other compounds using a standard analytical approach for all plant materials. J. Agric. Food Chem. 55 1084-1096.
Liorach, R., Espin, J.C., Tomas-Barberan, F.A., and Ferreres, F. (2002). Artichoke by-products as a potential source of health-promoting antioxidant phenolics. J. Agric. Food Chem. 50: 3458-3464.

Lipinski, C.A., Lombardo, F., Dominy, B.W., and Feeney, P.J. (2001). Experimental and computational approaches to estimate solubility and permeability in drug discovery and development settings. Adv. Drug Deliv. Rev. 46(1-3): 3-26.

Lisinska, G., and Leszczynski, W. (1987). Potato tubers as raw material for processing and nutrition. Potato Science and Technology. In: Lisinska, G., and Lesaczynski, W. (Ed.). Elsevier Applied Science, London, UK, 34-38.

Liyana-Pathirana, C.M., and Shahidi, F. (2006). Importance of insolublebound phenolics to antioxidant properties of wheat. J. Agric. Food Chem. 54: 1256-1264.

Liyana-Pathirana, C.M., and Shahidi, F. (2007). Antioxidant and free radical scavenging activities of whole wheat and milling fractions. Food Chem. 101: 1151-1157.

Llorach, R., Espín, J.C., Tomás-Barberán, F.A., and Ferreres, F. (2002). Artichoke (Cynara scolymus L.) byproducts as a potential source of health-promoting antioxidant phenolics. J. Agric. Food Chem. 50: 3458-3464.

Llorach, R., Espín, J.C., Tomás-Barberán, F.A., and Ferreres, F. (2003). Valorization of cauliflower (Brassica oleracea L. var. botrytis) by-products as a source of antioxidant phenolics. J. Agric. Food Chem. 51: 2181-2187.

Llorach, R., Tomás-Barberán, F.A., and Ferreres, F. (2004). Lettuce and chicory byproducts as a source of antioxidant phenolic extracts. J. Agric. Food Chem. 52: 5109-5116.

Loelillet, D. (1994). The European mango market: a promising tropical fruit. Fruit 49: 332-334.

Lommen, A., Godejohann, M., Venema, D.P., Hollman, P.C.H., and Spraul, M. (2000). Application of directly coupled HPLC-NMR-MS to the identification and confirmation of quercetin glycosides and phloretin glycosides in apple peel. Anal. Chem. 72: 1793-1797.

Lou, H.X., Yamazaki, Y., Sasaki, T., Uchida, M., and Tanaka, H. (1999). A-type procyanidins from peanut skin. Phytochemistry 51: 297-308.

Lu, Y., and Foo, L.Y. (1997). Identification and quantification of major polyphenols in apple pomace. Food Chem. 59: 187-194.

Lu, Y., and Foo, L.Y. (1998). Constitution of some chemical components of apple seed. Food Chem. 61: 29-33.

Lu, Y., and Foo, L.Y. (1999). The polyphenol constituents of grape pomace. Food Chem. 65: 1-8.

Lu, Y., and Foo, L. (2000). Antioxidant and radical scavenging activities of polyphenols from apple pomace. Food Chem. 68: 81-85.

Ludwig, I.A., Paz de Peña, M., Concepción, C., and Alan, C. (2013). Catabolism of coffee chlorogenic acids by human colonic microbiota. Biofac tors 39(6): 623-632.

Lyon, G.D., and Barker, H. (1984). The measurement of chlorogenic acid in potato leaf extracts by high pressure liquid chromatography. Potato Res. 27: 291-295.

Ma, Y.Q., Chen, J.C., Liu, D.H., and Ye, X.Q. (2009). Simultaneous extraction of phenolic compounds of citrus peel extracts: Effect of ultrasound. Ultrasonics Sonochem. 16: 57-62.

Macheix, J.J., Fleuriet, A., and Billot, J. (1990). Fruit Phenolics. CRC Press, Boca Raton, FL, pp. 1-126.

Madhujith, T., and Shahidi, F. (2005a). Antioxidant potential of pea beans (Phaseoul vulgaris L). J. Food Sci. 70: S85-S90.

Madhujith, T., and Shahidi, F. (2005b). Beans: A source of natural antioxidants. Phenolic Compounds in Food and Natural Health Products. In Shahidi, F., and Ho, C.T. (Ed.). American Chemical Society, USA, pp. 83-93.

Madhujith, T., Naczk, M., and Shahidi, F. (2004). Antioxidant activity of common beans (Phaseoul vulgaris L). J. Food Lipids 11: 220-233.

Maia, F.J.N., Ribeiro, F.W.P., Rangel, J.H.G.D., Lomonaco, D., Luna, F.M.T., de Lima-Neto, P., Correia, A.N., and Mazzetto, S.E. (2015). Evaluation of antioxidant action by electrochemical and accelerated oxidation experiments of phenolic compounds derived from cashew nut shell liquid. Indust. Crops and Prod. 67: 281-286.

Maier, T., Schieber, A., and Kammerer, D.R. (2009). Reinhold Carle Residues of grape (Vitis vinifera L.) seed oil production as a valuable source of phenolic antioxidants. Food Chem. 112: 551-559. 
Majima, T., Tsutsumi, M., Nishino, H., Tsunoda, T., and Konishi, Y. (1998). Chempreventive effects of green tea polyphenols. Pancreas 16: 1318.

Makris, D.P., and Rossiter, J.T. (2001). Domestic processing of onion bulbs and asparagus spears: Effect of flavonol content and antioxidant status. J. Agric. Food Chem. 49: 3216-3222.

Malik, A., and Mukhtar, H. (2006). Prostate cancer prevention through pomegranate fruit. Cell Cycle 5: 371-373.

Malmberg, A.G., and Theander, O. (1985). Determination of chlorogenic acid in potato tubers. J. Agric. Food Chem. 33: 549-551.

Manach, C., Mazur, A., and Scalbert, A. (2005b). Polyphenols and prevention of cardiovascular diseases. Curr. Opin. Lipidol. 16: 77-84.

Manach, C., Morand, C., Gil-Izquierdo, A., Bouteloup-Demange, C., and Remesy, C. (2003). Bioavailability in humans of the flavanones hesperidin and narirutin after the ingestion of two doses of orange juice. Eur. J. Clin. Nutr. 57: 235-242.

Manach, C., Morand, C., Gil-Izquierdo, A., Bouteloup-Demange, C., and Rémésy, C. (2003). Bioavailability in humans of the flavanones hesperidin and narirutin after the ingestion of two doses of orange juice. Eur. J. Clin. Nutr. 57: 235-242.

Manach, C., Scalbert, A., Morand, C., Rémésy, C., and Jiménez, L. (2004). Polyphenols: food sources and bioavailability. Am. J. Clin. Nutr. 79: 727-747.

Manach, C., Williamson, G., Morand, C., Scalbert, A., and Rémésy, C. (2005a). Bioavailability and bioefficacy of polyphenols in humans. I. Review of 97 bioavailability studies. Am. J. Clin. Nutr. 81: 230S-242S.

Mandalari, G., Bennett, R.N., Bisignano, G., Saija, A., Dugo, G., Curto, R.B.L., Faulds, C.B., and Waldron, K.W. (2006). Characterization of flavonoids and pectins from bergamot peel, a major by-product of essential oil extraction. J. Agric. Food Chem. 54: 197-203.

Manthey, A., and Grohmann, K. (1996). Concentrations of hesperidin and other orange peel flavonoids on citrus processing by-products. J. Agric. Food Chem. 44: 811-814.

Manthey, A., and Grohmann, K. (2001). Phenols in citrus peel byproducts. Concentrations of hydroxycinnamates and polymethoxylated flavones in citrus peel molasses. J. Agric. Food Chem. 49: 3268-3273.

Manthey, J.A., Grohmann, K., and Guthrie, N. (2001). Biological properties of citrus flavonoids pertaining to cancer and inflammation. Curr. Med. Chem. 8: 135-153.

Marais, J.P., Deavours, B., Dixon, R.A., and Ferreira, D. (2006). The Stereochemistry of the Flavonoids. The science of flavonoids. In: Grotewold, E. (Ed.). Springer science and Business Media, Inc, Columbus, Ohio, USA, pp. 1-46.

Martin, A.R., Villegas, I., La Casa, C., and de la Lastra, C.A. (2004). Resveratrol, a polyphenol found in grapes, suppresses oxidative damage and stimulates apoptosis during early colonic inflammation in rats. Biochem. Pharmacol. 67: 1399-1410.

Martin-Carron, N., Garcia-Alonso, A., Goni, I., and Saura-Calixto, F. (1997). Nutritional and physiological properties of grape pomace as a potential food ingredient. Americ. J. Enol. Viticulture 48: 328-332.

Martinez, G., Delgado, R., Perez, G., Garrido, G., Nunez-Selles, A.J., and Leon, O.S. (2000). Evaluation of the in vitro antioxidant activity of Mangifera indica L. extract (VIMANG). Phyto. Res. 14: 424-427.

Martínez-Valverde, I., Periago, M.J., Provan, G., and Chesson, A. (2002). Phenolic compounds, lycopene and antioxidant activity in commercial varieties of tomato (Lycopersicon esculentum). J. Sci. Food Agric. 82: $323-330$.

Mathew, A.G., and Parpia, H.A.B. (1970). Polyphenols of cashew skin. J. Food Sci. 35: 140-143.

Mathew, S., and Abraham, T.E. (2004). Ferulic acid: An antioxidant found naturally in plant cell walls and feruloyl esterases involved in its release and their applications. Crit. Rev. Biotechnol. 24: 59-83.

Matsumoto, H., Inaba, H., Kishi, M., Tominaga, S., Hirayama, M., and Tsuda, T. (2001). Orally administered delphinidin 3-rutinoside and cyanidin 3-rutinoside are directly absorbed in rats and humans and appear in the blood as the intact forms. J. Agric. Food Chem. 49: 1546-1551.

Mattila, P., and Hellström, J. (2007). Phenolic acids in potatoes, vegetables, and some of their products. J. Food Compos. Anal. 20: 152-160.

Mazur, W.M. (1998). Phytoestrogen content in foods. Bailliêre's clinical endocrinology and metabolism: phyto-oestrogens. In: Adlercreutz, H. (Ed.). Baillière Tindall, London, UK, pp. 729-742.
Mazur, W., Wähälä, K., Rasku, S., Salakka, A., Hase, T., and Adlercreutz, H. (1998). Lignan and isoflavonoid concentrations in tea and coffee. Brit. J. Nutrition 79: 37-45.

Mazza, G. (1995). Anthocyanins in grapes and grape products. Crit. Rev. Food Sci. Nutr. 35: 341-371.

Mazza, G., and Miniati, E. (1993). Grapes. Anthocyanins in fruits, vegetables, and grains. RC Press, Boca Raton, FL, pp. 149-199.

McClean, P.E., Lee, R.K., Otto, C., Gepts, P., and Bassett, M.J. (2002). Molecular and phenotypic mapping of genes controlling seed coat pattern and colour in common bean (Phaseolus vulgaris L.). J. Hered. 93: 148-152.

McDougall, B., King, P.J., Wu, B.W., Hostomsky, Z., Reinecke, M.G., and Robinson, W.E. (1998). Dicaffeoylquinic and dicaffeoyltartaric acids are selective inhibitors of human immunodeficiency virus type 1 integrase. Antimicrob. Agents Chemother. 42: 140-146.

McGhie, T.K., and Walton, M.C. (2007). The bioavailability and absorption of anthocyanins: Towards a better understanding. Mol. Nutr. Food Res. 51: 702-713.

McGhie, T.K., Ainge, G.D., Barnett, L.E., Cooney, J.M., and Jensen, J.D. (2003). Anthocyanin glycosides from berry fruit are absorbed and excreted unmetabolized by both humans and rats. J. Agric. Food Chem. 51: 4539-4548.

Meng, X., Maliakal, P., Lu, H., Lee, M.J., and Yang, C.S. (2004). Urinary and plasma levels of resveratrol and quercetin in humans, mice, and rats after ingestion of pure compounds and grape juice. J. Agric. Food Chem. 52: 935-942.

Meng, X., Sang, S., Zhu, N., Lu, H., Sheng, S., Lee, M.J., Ho, C.T., and Yang, C.S. (2002). Identification and characterization of methylated and ring-fission metabolites of tea catechins formed in humans, mice, and rats. Chem. Res. Toxicol. 15: 1042-1050.

Meng, Z., Sun, L.H., Chen, Y.Y., Ye, M., Su, H., Xing, D.M., and Du, L.J. (2005). The effect of pomegranate leaf tannide on hyperlipidemic models. Chin. J. Experi. Trad. Med. Formulae 11: 22-24.

Merken, H.M., and Beecher, G.R. (2000). Measurement of food flavonoids by high-performance liquid chromatography: a review. J. Agric. Food. Chem. 48: 577-599.

Meshkini, A. (2016). Acetone extract of almond hulls provides protection against oxidative damage and membrane protein degradation. J. Acupunct. Meridian Stud. 9(3): 134-142.

Meyer, A.S., Jepsen, S.M., and Sorensen, N.S. (1998). Enzymatic release of antioxidants for human low-density lipoprotein from grape pomace. J. Agric. Food Chem. 46: 2439-2446.

Middleton, E., Kandaswami, C., and Theoharides, T.C. (2000). The effects of plant flavonoids on mammalian cells: implications for inflammation, heart disease and cancer. Pharmacol. Rev. 52: 673-751.

Milbury, P.E., Cao, G., Prior, R.L., and Blumberg, J. (2002). Bioavailability of elderberry anthocyanins. Mech. Ageing Develop. 123: 997-1006.

Milbury, P., Chen, C.Y., Dolnikowski, G., and Blumberg, J.B. (2006). Determination of flavonoids and phenolics and their distribution in almonds. J. Agric. Food Chem. 54: 5027-5033.

Miró-Casas, E., Covas, M.-I., Farré, M., Fito, M., Ortuño, J., Weinbrenner, T., Roset, P., and Torre, R. (2003a). Hydroxytyrosol disposition in humans. Clin. Chem. 49: 945-952.

Miró-Casas, E., Covas, M.-I., Fito, M., Farré-Albaladejo, M., Marrugat, J., and Torre, R. (2003b). Tyrosol and hydroxytyrosol are absorbed from moderate and sustained doses of virgin olive oil in humans. Eur. J. Clin. Nutr. 57: 186-190.

Miró-Casas, E., Farré-Albaladejo, M., Covas, M.-I., Ortuño-Rodriguez, J., Menoyo-Colomer, E., Lamuela-Raventós, R.M., and Torre, R. (2001). Capillary gas chromatography-mass spectrometry quantitative determination of hydroxytyrosol and tyrosol in human urine after olive oil intake. Anal. Biochem. 294: 63-72.

Mitaru, B.N., Blair, R., Bell, J.M., and Reichert, R.D. (1982). Tannin and fiber contents of rapeseed and canola hulls. Can. J. Anim. Sci. 62: 661-663.

Mitscher, L., Jung, M., Shankel, D., Dou, J.H., Steele, L., and Pillai, S.P. (1997). Chemoprotection: A review of the potential therapeutic antioxidant properties of green tea and certain of its constituents. Med. Res. Rev. 17: 327-365.

Miyake, Y., Yamamoto, K., Morimitsu, Y., and Osawa, T. (1997). Isolation of C-glucosylflavone from lemon peel and antioxidative activity of flavonoid compounds in lemon fruit. J. Agric. Food Chem. 45: 4619-4623. 
Miyazawa, T., Nakagawa, K., Kudo, M., Muraishi, K., and Someya, K. (1999). Direct intestinal absorption of red fruit anthocyanins, cyanidin-3-glucoside and cyanidin-3,5-diglucoside, into rats and humans. J. Agric. Food Chem. 47: 1083-1091.

Mohsen, A.E.M.M., Kuhnle, G., Rechner, A.R., Schroeter, H., Rose, S., Jenner, P., and Rice-Evans, C.A. (2002). Uptake and metabolism of epicatechin and its access to the brain after oral ingestion. Free Radic Bio Med. 33: 1693-1702.

Mohsen, S.M., and Ammar, A.S.M. (2009). Total phenolic contents and antioxidant activity of corn tassel extracts. Food Chem. 112: 595-598.

Monagas, M., Garrido, L., Lebron-Aguilar, R., Bartolome, B., and GomezCordoves, C. (2007). Almond skins as a potential source of bioactive polyphenols. J. Agric. Food Chem. 55: 8498-8507.

Moosavi, K.D., Dehghan, G., Hosseini, S., and Jahanban, A.E. (2015). Effect of five year storage on total phenolic content and antioxidant capacity of almond (Amygdalus communis L.) hull and shell from different genotypes. Avicenna J. Phytomed. 5: 26-33.

Morello, M.J., Shahidi, F., and Ho, C.T. (2002). Free radicals in food: Chemistry, nutrition and health effect. ACS symposium series 807: 1-9.

Morton, L.W., Caccetta, R.A., Puddey, I.B., and Croft, K.D. (2000). Chemistry and biological effects of dietary phenolic compounds: Relevance to cardiovascular disease. Clin. Exp. Pharmacol. Physiol. 27: 152-159.

Mosele, J.I., Macià, A., and Motilva, M.-J. (2015). Metabolic and microbial modulation of the large intestine ecosystem by non-absorbed diet phenolic compounds: A review. Molecules 20(9): 17429-17468.

Moss, G.P. (2000). Nomenclature of Lignans and Neolignans. Pure Appl. Chem. 72: 1493-1523.

Mouly, P.P., Arzouyan, C.R., Gaydou, E.M., and Estienne, J.M. (1994). Differentiation of citrus juices by factorial discriminant analysis using liquid chromatography of flavanone glycosides. J. Agric. Food Chem. 42: 70-79.

Moure, A., Cruz, J.M., Franco, D., Domínguez, J.M., Sineiro, J., Domínguez, H., Núñez, M.J., and Parajó, J.C. (2001). Natural antioxidants from residual sources, a review. Food Chem. 72: 145-171.

Mullen, W., Archeveque, M.-A., Edwards, C.A., Matsumoto, H., and Crozier, A. (2008). Bioavailability and metabolism of orange juice flavanones in humans: Impact of a full-fat yogurt. J. Agric. Food Chem. 56: 11157-11164.

Murkovic, M., Mülleder, U., Adam, U., and Pfannhauser, W. (2001). Detection of anthocyanins from elderberry juice in human urine. J. Sci. Food Agric. 81: 934-937.

Murphy, K.J., Chronopoulos, A.K., Singh, I., Maureen, A.F., Helen, M., Marilyn, P., Alan, H.T., Neil, J.M., and Andrew, J.S. (2003). Dietary flavanols and procyanidins oligomers from cocoa (Theobroma cacao) inhibit platelet function. Am. J. Clin. Nutr. 77: 1466-1473.

Murthy, K.N.C., Jayaprakasha, G.K., and Singh, R.P. (2002). Studies on antioxidant activity of pomegranate (Punica granatum) peel extract using in vivo models. J. Agric. Food Chem. 50: 4791-4795.

Naasani, I., Seimiya, H., and Tsuruo, T. (1998). Telomerase Inhibition, Telomere Shortening, and Senescence of Cancer Cells by Tea Catechins. Biochem. Biophys. Res. Commun. 249: 391-396.

Naczk, M., Amarowicz, R., Pink, D., and Shahidi, F. (2000). Insoluble condensed tannins of canola and rapeseed hulls. J. Agric. Food Chem. 48: 1758-1762.

Naczk, M., Nichols, T., Pink, D., and Sosulski, F. (1994). Condensed Tannins in Canola Hulls. J. Agric. Food Chem. 42: 2196-2200.

Naczk, M., Oickle, D., Pink, D., and Shahidi, F. (1996). Protein precipitating capacity of crude canola tannins: effect of $\mathrm{pH}$, tannin, and protein concentrations. J. Agric. Food Chem. 44: 2144-2148.

Naczk, M., Pegg, R.B., Zadernowski, R., and Shahidi, F. (2005). Radical scavenging activity of canola hull phenolics. J. Am. Oil Chem. Soc. 82: $255-260$.

Naczk, M., and Shahidi, F. (1998). Insoluble condensed tannins of canola. Book of Abstracts of the 58th Annual IFT Meeting. Atlanta, GA

Narasimha Char, B.L., and Azeemoddin, G. (1989). Edible fat from mango stones. Acta Horticulture 231: 744-748.

Nardini, M., Cirillo, E., Natella, F., and Scaccini, C. (2002). Absorption of phenolic acids in humans after coffee consumption. J. Agric. Food Chem. 50: 5735-5741.

Nardini, M., D’Aquino, M., Tomassi, G., Gentilli, V., Di Felice, M., and Scaccini, C. (1995). Inhibition of human low-density lipoprotein oxidation by caffeic acid and other hydroxycinnamic acid derivatives. Free Radic. Biolog. Med. 19: 541-552.

Natella, F., Belelli, F., and Gentili, V. (2002). Grape Seed Proanthocyanidins Prevent Plasma Postprandial Oxidative Stress in Humans. J. Agri. Food Chem. 50: 7720-7725.

Naurato, N., Wong, P., Lu, Y., Wroblewski, K., and Bennick, A. (1999). Interaction of tannin with human salivary histatins. J. Agric. Food Chem 47: 2229-2234.

Nawaz, H., Shi, J., Mittal, G.S., and Kakuda, Y. (2006). Extraction of polyphenols from grape seeds and concentration by ultrafiltration. Sep. Purifi. Technol. 48: 176-181.

Negi, P.S., and Roy, S.K. (2001). The effect of blanching on quality attributes of dehydrated carrots during long-term storage. Eur. Food Res. and Tech. 212: 445-448.

Negi, P.S., Jayaprakasha, G.K., and Jena, B.S. (2003). Antioxidant and antimutagenic activities of pomegranate peel extracts. Food Chem. 80 393-397.

Nepote, V., Grosso, N.R., and Guzman, C.A. (2002). Extraction of antioxidant components from peanut skins. Grasas Y. Aceites 53: 391-395.

Nesbitt, P.D., and Thompson, L.U. (1997). Lignans in homemade and com mercial products containing flaxseed. Nutr. Cancer 29: 222-227.

Nichenametla, S.N., Taruscio, T.G., Barney, D.L., and Exon, J.H. (2006). A review of the effects and mechanisms of polyphenolics in cancer. Crit. Rev. Food Sci. Nutr. 46: 161-183.

Nicholson, R.L., and Hammerschmidt, R. (1992). Phenolic compounds and their role in disease resistance. Annu. Rev. Phytopathol. 30: 369-389.

Nicolosi, R.J., Rogers, E.J., Ausman, L.M., and Orthoefer, F.T. (1994). Rice bran oil and its health benefits. Rice Science and Technology. In: Marshall, W.E., and Wadsworth, J.I. (Ed.). Marcel Dekker, New York, NY, 350-421.

Nielsen, I.L.F., Chee, W.S.S., Poulsen, L., Offord-Cavin, E., Rasmussen, S.E. Frederiksen, H., Enslen, M., Barron, D., Horcajada, M.N., and Williamson, G. (2006). Bioavailability is improved by enzymatic modification of the citrus flavonoid hesperidin in humans: A randomized, double-blind, crossover trial. J. Nutr. 136: 404-408.

Nilnakara, S., Chiewchan, N., and Devahastin, S. (2009). Production of antioxidant dietary fibre powder from cabbage outer leaves. Food Bioprod. Process. 87: 301-307.

Nilsson, J., Stegmark, R., and Akesson, B. (2004). Total antioxidant capacity in different pea (Pisum sativum) varieties after blanching and freezing. Food Chem. 86: 501-507.

Ninfali, P., and Angelino, D. (2013). Nutritional and functional potential of Beta vulgaris cicla and rubra. Fitoterapia 89: 188-199.

Niño-Medina, G., Muy-Rangel, D., de la Garza, A.L., Rubio-Carrasco, W., Pérez-Meza, B., Araujo-Chapa, A.P., Gutiérrez-Álvarez, K.A., and Urías-Orona, V. (2019). Dietary fiber from chickpea (Cicer arietinum) and soybean (Glycine max) husk byproducts as baking additives: Functional and nutritional properties. Molecules 24: 991.

Noguchi, Y., Fukuda, K., Matsushima, A., Haishi, D., Hiroto, M., Kodera, Y., Nishimura, H., and Inada, Y. (1999). Inhibition of Df-protease associated with allergic diseases by polyphenol. J. Agric. Food Chem. 47: 2969-2972.

Nunez-Selles, A.J. (2005). Antioxidant therapy: myth or reality? J. Brazil. Chem. Soc. 16: 699-710.

Nurmi, T., Heinonen, S., Mazur, W., Deyama, T., Nishibe, S., and Adlercreutz, H. (2003). Lignans inselected wines. Food Chem. 83: 303-309.

Nwuha, V., Nakajima, M., Tong, J., and Ichikawa, S. (1999). Solubility study of green tea extracts in pure solvents and edible oils. J. Food Eng. 40: 161-165.

Obied, H.K., Allen, M.S., Bedgood, D.R., Prenzler, P.D., Robards, K., and Stockmann, R. (2005). Bioactivity and analysis of biophenols recovered from olive mill waste. J. Agric. Food Chem. 53: 823-837.

Oguni, I., Chen, S.J., Lin, P.Z., and Hara, Y. (1992). Protection Against Cancer Risk by Japanese Green Tea. Prev. Med. 21: 332-333.

Oh, W.Y., and Shahidi, F. (2017). Lipophilization of resveratrol and effects on antioxidant activities. J. Agric. Food Chem. 65: 8617-8625.

Oh, W.Y., and Shahidi, F. (2018). Antioxidant activities of resveratrol ester derivatives in food and biological model systems. Food Chem. 261: 267-273

Oldoni, T.L., Melo, P.S., Massarioli, A.P., Moreno, I.A., Bezerra, R.M., Rosalen, P.L., da Silva, G.V., Nascimento, A.M., and Alencar, S.M. (2016) 
Bioassay-guided isolation of proanthocyanidins with antioxidant activity from peanut (Arachis hypogaea) skin by combination of chromatography techniques. Food Chem. 192: 306-312.

Olthof, M.R., Hollman, P.C.H., and Katan, M. (2001). Chlorogenic acid and caffeic acid are absorbed in humans. Human Nutr. Metabol. 131: 66-71.

Olthof, M.R., Hollman, P.C.H., Buijsman, M.N.C.P., van Amelsfoort, J.M., and Katan, M.B. (2003). Chlorogenic acid, quercetin-3-rutinoside, and black tea phenols are extensively metabolized in humans. J. Nutr. 133: 1806-1814.

Olthof, M., Hollman, P.C.H., Vree, T.B., and Katan, M.B. (2000). Bioavailabilities of quercetin-3-glucoside and quercetin-4'-glucoside do not differ in humans. J. Nutr. 130: 1200-1203.

Onyeneho, S.N., and Hettiarachchy, N.S. (1992). Antioxidant activity of durum wheat bran. J. Agric. Food Chem. 40: 1496-1500.

Onyeneho, S.N., and Hettiarachchy, N.S. (1993). Antioxidant activity, fatty acids and phenolic acids composition of potato peels. J. Sci. Food Agric. 62 : $345-350$.

Oomah, B.D., and Mazza, G. (1999). Health benefits of phytochemicals from selected Canadian crops. Trends Food Sci. Technol. 10: 193-198.

Osawa, T., Narasimhan, R., Kawakishi, S., Namiki, M., and Tashiro, T. (1985). Antioxidant defense system in rice hull against damage caused by oxygen radicals. Agric. Biol. Chem. 10: 3085-3087.

Osman, H., Nasarudin, R., and Lee, S.L. (2004). Extracts of cocoa (Theobroma cacao L.) leaves and their antioxidation potential. Food Chem. 86: 41-46.

Ososki, A.L., and Kennelly, E.J. (2003). Phytoestrogens: a review of the present state of research. Phytother-Res. 17: 845-869.

Otto, K., and Sulc, D. (2001). Herstellung von Gemusesaften. Frucht- und Gemusesafte. In: Schobinger, U. (Ed.). Stuttgart, 278-297.

Ou, M. (1999). Regular Chinese Medicine Handbook. Warmth Publishing Ltd., Taiwan.

Ou, S.Y., and Kwok, K.C. (2004). Review. Ferulic acid: pharmaceutical functions, preparation and applications in foods. J. Sci. Food Agric. 84: 1261-1269.

Oufedjikh, H., Mahrouz, M., Amiot, M.J., and Lacroix, M. (2000). Effect of gamma-irradiation on phenolic compounds and phenylalanine ammonia-lyase activity during storage in relation to peel injury from peel of Citrus clementina Hort. ex. Tanaka. J. Agric. Food Chem. 48: 559-565.

Pace-Asciak, C.R., Hahn, S.E., Diamandis, E.P., Soleas, G.J., and Goldberg, D.M. (1995). The red wine phenolics trans-resveratrol and quercetin block human platelet aggregation and eicosanoid synthesis: Implications for protection against coronary heart disease. Clinica Chimica Acta 235: 207-219.

Palma, M., and Taylor, L.T. (1999). Extraction of polyphenolic compounds from grape seeds with near critical carbon dioxide. J. Chromatogr. A 849: 117-124.

Palomo, I., Concha-Meyer, A., Lutz, M., Said, M., Sáez, B., Vásquez, A., and Fuentes, E. (2019). Chemical characterization and antiplatelet potential of bioactive extract from tomato pomace (byproduct of tomato paste). Nutrients 11: 456

Pan, X., Niu, G., and Liu, H. (2003). Microwave-assisted extraction of tea polyphenols and tea caffeine from green tea leaves. Chem. Eng. Process. 42: 129-133.

Pantuck, A.J., Leppert, J.T., Zomorodian, N., Aronson, W., Hong, J., Barnard, R.J., Seeram, N., Liker, H., Wang, H., Elashoff, R., Heber, D., Aviram, M., Ignarro, L., and Belldegrun, A. (2006). Phase II study of pomegranate juice for men with rising prostatespecific antigen following surgery or radiation for prostate cancer. Clin. Cancer Res. 12: 40184026 .

Pardo-Andreu, G.L., Philip, S.J., Riano, A., Sanchez, C., Viada, C., NunezSelles, A.J., and Delgado, R. (2006). Mangifera indica L. (Vimang) protection against serum oxidative stress in elderly humans. Arch. Med. Res. 37: 158-164.

Park, Y.C., Rimbach, G., Saliou, C., Valacchi, G., and Packer, L. (2000). Activity of monomeric, dimeric, and trimeric flavonoids on NO production, TNF-alpha secretion, and NF-kappaB-dependent gene expression in RAW 264.7 macrophages. FEBS Letters 465: 93-97.

Pasqualone, A., Delvecchio, L.N., Gambacorta, G., Laddomada, B., Urso, V., Mazzaglia, A., Ruisi, P., and di Miceli, G. (2015). Effect of supplemen- tation with wheat bran aqueous extracts obtained by ultrasoundassisted technologies on the sensory properties and the antioxidant activity of dry pasta. Nat. Prod. Commun. 10: 1739-1742.

Pazmino-Duran, E.A., Giusti, M.M., Wrolstad, R.E., and Gloria, M.B.A. (2001). Anthocyanins from banana bracts (Musa paradisiaca) as potential food colourant. Food Chem. 73: 327-332.

Pedreno, M.A., and Escribano, J. (2001). Correlation between antiradical activity and stability of betanine from Beta vulgaris $L$ roots under different $\mathrm{pH}$, temperature and light conditions. J. Sci. Food Agric. 81: 627-631.

Pedrosa, M.M., Muzquiz, M., Garcia-Vallejo, C., Burbano, C., Cuadrado, C., Ayet, G., and Robredo, L.M. (2000). Determination of caffeic and chlorogenic acids and their derivatives in different sunflower seeds. J. Sci. Food Agric. 80: 459-464.

Peng, H., Deng, Z., Chen, X., Sun, Y., Zhang, B., and Li, H. (2018). Major chemical constituents and antioxidant activities of different extracts from the peduncles of Hovenia acerba Lindl. Int. J. Food Prop. 21(1): 2135-2155.

Peng, H., Li, W., Li, H., Deng, Z., and Zhang, B. (2017). Extractable and nonextractable bound phenolic compositions and their antioxidant properties in seed coat and cotyledon of black soybean (Glycine max (L.) merr). J. Funct. Foods 32: 296-312.

Peričin, D., Krimer, V., Trivić, S., and Radulović, L. (2009). The distribution of phenolic acids in pumpkin's hull-less seed, skin, oil cake meal, dehulled kernel and hull. Food Chem. 113: 450-456.

Peschel, W., Sanchez-Rabaneda, F., Diekmann, W., Plescher, A., Gartzı, I., Jimenez, D., Lamuela-Ravento, R., Buxaderas, S., and Codina, C. (2006). An industrial approach in the search of natural antioxidants from vegetable and fruit wastes. Food Chem. 97: 137-150.

Peterson, J.J., Beecher, G.R., Bhagwat, S.A., Dwyer, J.T., Gebhardt, S.E., Haytowitz, D.B., and Holden, J.M. (2006). Flavanones in grapefruit, lemons, and limes: A compilation and reviewof the data from the analytical literature. J. Food Comp. Anal. 19: S74-S80.

Pezet, R., and Pont, P. (1988). Mise en evidence de pterostilbene dans les grappes de Vitis vinifera. Plant Physiol. Biochem. 26: 603-607.

Pezet, R., Perret, C., Jean-Denis, J.B., Tabacchi, R., Gindro, K., and Viret, O. (2003). $\delta$-Viniferin, a resveratrol dehydrodimer: One of the major stilbenes synthesized by stressed grapevine leaves. J. Agric. Food Chem. 51: 5488-5492.

Pihlanto, A., Akkanen, S., and Korhonen, H.J. (2008). ACE-inhibitory and antioxidant properties of potato (Solanum tuberosum). Food Chem. 109: 104-112.

Pillai, M.K.S., Kedlaya, K.J., and Selvarangan, R. (1963). Cashew seed skin as a tanning material. Leather Sci. 10: 317.

Piskula, M.K., and Terao, J. (1998). Accumulation of (-)-epicatechin metabolites in rat plasma after oral administration and distribution of conjugation enzymes in rat tissues. J. Nutr. 128: 1172-1178.

Plastina, P., Benincasa, C., Perri, E., Fazio, A., Augimeri, G., Poland, M., Witkamp, R., and Meijerink, J. (2019). Identification of hydroxytyrosyl oleate, a derivative of hydroxytyrosol with anti-inflammatory properties, in olive oil by-products. Food Chem. 279: 105-113.

Poquet, L., Clifford, M.N., and Williamson, G. (2008). Transport and metabolism of ferulic acid through the colonic epithelium. Drug Metab. Dispos. 36: 190-197.

Porter, L.J. (1989). Tannins. Methods in Plant Biochemistry: Vol. 1. Plant Phenolics. In: Harborne, J.B. (Ed.). Academic press, London, UK, pp. 389-419.

Porter, L.J. (1993). Flavans and proanthocyanidins. The Flavonoids. Advances in Research since 1986 1993. In: Harborne, J.B. (Ed.). Chapman and Hall, London, UK, pp. 23-55.

Prakash, D., Singh, B.N., and Upadhyay, G. (2007). Antioxidant and free radical scavenging activities of phenols from onion (Allium cepa). Food Chem. 102: 1389-1393.

Prgomet, I., Gonçalves, B., Domínguez-Perles, R., Pascual-Seva, N., and Barros, A. (2017). Valorization challenges to almond residues: Phytochemical composition and functional application. Molecules 22(10): 1774.

Price, K.R., and Rhodes, M.J.C. (1997). Analysis of the major flavonol glycosides present in four varieties of onion (Allium cepa) and changes in composition resulting from autolysis. J. Sci. Food Agric. 74: 331-339.

Pridham, J.B. (1960). Phenolics in Plants in Health and Disease. Pergamon 
Press, New York, NY

Prieur, C., Rigaud, J., Cheynier, V., and Moutounet, M. (1994). Oligomeric and polymeric procyanidins from grape seeds. Phytochemistry 36 781-784.

Prior, R.L., and Wu, X. (2006). Anthocyanins: structural characteristics that result in unique metabolic patterns and biological activities. Free Rad. Res. 40: 1014-1028.

Püssa, T., Floren, J., Kuldkepp, P., and Raal, A. (2006). Survey of grapevine stem polyphenols by liquid chromatography-diode array detectiontandem mass spectrometry. J. Agric. Food Chem. 54: 7488-7494.

Puravankara, D., Boghra, V., and Sharma, R.S. (2000). Effect of antioxidant principles isolated from mango (Mangifera indica L.) seed kernels on oxidative stability of buffalo ghee (butter-fat). J. Sci. Food Agric. 80 522-526.

Puupponen-Pimiä, R., Nohynek, L., Meier, C., Kähkönen, M., Heinonen, M., Hopia, A., and Oksman-Caldentey, K.M. (2001). Antimicrobia properties of phenolic compounds from berries. J. App. Microbiol. 90: 494-507.

Pyo, Y.H., Lee, T.C., Logendra, L., and Rosen, R.T. (2004). Antioxidant activity and phenolic compounds of Swiss chard (Beta vulgaris subspecies cycla) extracts. Food Chem. 85(1): 19-26.

Qiu, S.-X., Lu, Z.-Z., Luyengi, L., Lee, S.K., Pezzuto, J.M., Farnsworth, N.R., Thompson, L.U., and Fong, H.H.S. (1999). Isolation and Characterization of Flaxseed (Linum usitatissimum) Constituents. Pharm. Biol. 37: $1-7$.

Quiñones, M., Guerrero, L., Suarez, M., Pons, Z., Aleixandre, A., Arola, L., and Muguerza, B. (2013). Low-molecular procyanidin rich grape seed extract exerts antihypertensive effect in males spontaneously hypertensive rats. Food Res. Int. 51(2): 587-595.

Ralph, J., Bunzel, M., Marita, J.M., Hatfield, R.D., Lu, F., Kim, H., Schatz, P.F., Grabber, J.H., and Steinhart, H. (2004). Peroxidasedependent crosslinking reactions of $p$-hydroxycinnamates in plant cell walls. Phytochem. Rev. 3: 79-96.

Ramamurthy, M., Maiti, B., Thomas, P., and Nair, M. (1992). High performance liquid chromatography determination of phenolic acids in potato tubers (Solanum tuberosum) during wound healing. J. Agri. Food Chem. 40: 569-572.

Ramarathnam, N., Osawa, T., Namiki, M., and Kawakishi, S. (1989). Chemical studies on novel rice hull antioxidants. 2. Identification of isovitexin, a c-glycosyl flavonoid. J. Agric. Food Chem. 37: 316-319.

Ramos-Cormenzana, A., Juarez-Jimenez, B., and Garcia-Pareja, M.P. (1996). Antimicrobial activity of olive-mill waste waters (alpechin) and biotransformed olive oil mill wastewater. Int. Biodeterior. Biodegradation 38: 283-290.

Rangkadilok, N., Sitthimonchai, S., Worasuttayangkurn, L., Mahidol, C., Ruchirawat, M., and Satayavivad, J. (2007). Evaluation of free radical scavenging and antityrosinase activities of standardized longan fruit extract. Food Chem. Toxicol. 45: 328-336.

Ranilla, L.G., Genovese, M.I., and Lajolo, F.M. (2007). Polyphenols and antioxidant capacity of seed coat and cotyledon from Brazilian and Peruvian bean cultivars (Phaseolus vulgaris L.). J. Agric. Food Chem. 55: 90-98.

Rao, C.V., Desai, D., Simi, B., Kulharni, N., Amin, S., and Reddy, B.S. (1993). Inhibitory effect of caffeic acid esters on azoxymethane-induced biochemical changes and aberrent crytp foci, formation in rat colon Cancer Res. 53: 4182-4188.

Rechner, A.R., Smith, M.A., Kuhnle, G., Gibson, G.R., Debnam, E.S., Srai, S.K.S., Moore, K.P., and Rice-Evans, C.A. (2004). Colonic metabolism of dietary polyphenols: influence of structure on microbial fermentation products. Free Radical. Bio. Med. 36: 212-225.

Reis, S.F., Rai, D.K., and Abu-Ghannam, N. (2012). Water at room temperature as a solvent for the extraction of apple pomace phenolic compounds. Food Chem. 135: 1991-1998.

Renaud, S., and De Lorgeril, M. (1992). Wine, alcohol, platelets, and the French Paradox for coronary heart disease. Lancet 339: 1523-1526.

Richelle, M., Prodmore-Merten, S., Bodenstab, S., Enslen, M., and Offord, E.A. (2002). Hydrolysis of isoflavone glycosides to aglycones by $\beta$-glycosidase does not alter plasma and urine isoflavone pharmacokinetics in postmenopausal women. J. Nutr. 132: 2587-2592.

Riemersma, R.A., Rice-Evans, C.A., Tyrell, R.M., Clifford, M.N., and Lean, M.E. (2001). Tea flavonoids and cardiovascular health. Q. J. M. 94
277-282.

Riggi, E., and Avola, G. (2008). Fresh tomato packinghouses waste as high added-value biosource Resources. Conserv. Recy. 53: 96-106.

Rimm, E.B., Katan, M.B., Ascherio, A., Stampfer, M.J., and Willett, W.C. (1996). Relation between intake of flavonoids and risk of coronary heart disease in male health professionals. Ann Intern. Med. 125 384-389.

Robbins, R.J. (2003). Phenolic acids in foods: An overiew of analytical methodology. J. Agric. Food Chem. 51: 2866-2887.

Rockenbach, I.I., Gonzaga, L.V., Rizelio, V.M., Goncalves, A.E.d.S.S., Genovese, M.I.s., and Fett, R. (2011). Phenolic compounds and antioxidant activity of seed and skin extracts of red grape (Vitis vinifera and Vitis labrusca) pomace from Brazilian winemaking. Food Res. Int 44: 897-901.

Rodis, P.S., Karathanos, V.T., and Mantzavinou, A. (2002). Partitioning of olive oil antioxidants between oil and water phases. J. Agric. Food Chem. 50: 596-601.

Rodriguez, J., Pierro, D.D., Gioia, M., Monaco, S., Delgado, R., Coletta, M. and Marini, S. (2006). Effects of natural extract from Mangifera indica L., and its active compound, mangiferin, on energy state and lipid peroxidation of red blood cells. Biochemica et Biophysica Acta 1760: 1333-1342.

Rodríguez, R., Jiménez, A., Jaramillo, S., Guillén, R., Fernández-Bolaños, J., Rodríguez, G., and Heredia, A. (2004). Asparagus By-Product as Source of Functional Compounds. Total Food: Exploiting Co-ProductsMinimising Waste. In: Waldron, K.W. (Ed.). Institute of Food Research, Norwich, UK, pp. 110-115.

Rodriguez de Sotillo, D., Hadley, M., and Holm, E.T. (1994a). Phenolics in aqueous potato peel extract: extraction, identification and degradation. J. Food Sci. 59: 649-651.

Rodriguez de Sotillo, D., Hadley, M., and Holm, E.T. (1994b). Potato peel waste: Stability and antioxidant activity of a freeze-dried extract. J. Food Sci. 59: 1031-1033.

Rodriguez de Sotillo, D., Hadley, M., and Wolf-Hall, C. (1998). Potato peel extract a non-mutagenic antioxidant with potential antimicrobial activity. J. Food Sci. 63: 907-910.

Rodríguez Montealegre, R., Romero Peces, R., Chacón Vozmediano, J.L. Martínez Gascueña, J., and García Romero, E. (2006). Phenolic compounds in skins and seeds of ten grape Vitis vinifera varieties grown in a warm climate. J. Food Comp. Anal. 19: 687-693.

Rodríguez-Morató, J. (2016a). Metabolism of natural antioxidants: evaluation of the pathways involved in the in vivo biotransformation of tyrosol into hydroxytyrosol (Doctoral dissertation, Universitat Pompeu Fabra).

Rodríguez-Morató, J., Boronat, A., Kotronoulas, A., Pujadas, M., Pastor, A. Olesti, E., Perez-Mana, C., Khymenets, O., Fitó, M., Farré, M., and de la Torre, R. (2016b). Metabolic disposition and biological significance of simple phenols of dietary origin: Hydroxytyrosol and tyrosol. Drug Metab. Rev. 48: 218-236

Rodríguez-García, C., Sánchez-Quesada, C., Toledo, E., Delgado-Rodríguez, M., and Gaforio, J.J. (2019). Naturally lignan-rich foods: a dietary tool for health promotion? Molecules 24: 917.

Rodriguez-Saona, L.E., Giusti, M.M., and Wrolstad, R.E. (1998). Anthocyan pigment composition of red-fleshed potatoes. J. Food Sci. 63 458-465.

Rodriguez-Saona, L.E., Wrolstad, R.E., and Pereira, C. (1999). Glycoalkaloid content and anthocyanin stability to alkaline treatment of redfleshed potato extracts. J. Food Sci. 64: 445-450.

Rodsamrana, P., and Sothornvit, R. (2019). Extraction of phenolic compounds from lime peel waste using ultrasonicassisted and microwave-assisted extractions. Food Biosci. 28: 66-73.

Romani, A., Vignolini, P., Galardi, C., Mulinacci, N., Benedettelli, S., and Heimler, D. (2004). Germoplasm characterization of Zolfino landraces (Phaseolus vulgaris L.) by flavonoid content. J. Agric. Food Chem. 52 3838-3842.

Rotches-Ribalta, M., Andres-Lacueva, C., Estruch, R., Escribano, E., and Urpi-Sarda, M. (2012a). Pharmacokinetics of resveratrol metabolic profile in healthy humans after moderate consumption of red wine and grape extract tablets. Pharmacol. Res. 66(5): 375-382.

Rouseff, R.L., Martin, S.F., and Youtsey, C.O. (1987). Quantitative survey of narirutin, naringin, hesperidin and neohesperidin in Citrus. J. Agric. 
Food Chem. 35: 1027-1030

Rüfer, C.E., Bub, A., Möseneder, J., Winterhalter, P., Stürtz, M., and Kulling, S.E. (2008). Pharmacokinetics of the soybean isoflavone daidzein in its aglycone and glucoside form: a randomized, double-blind, crossover study. Am. J. Clin. Nutr. 87: 1314-1323.

Ruiz-Cano, D., Pérez-Llamas, F., Frutos, M.J., Arnao, M.B., Espinosa, C., López-Jiménez, J.A., Castillo, J., and Zamora, S. (2014). Chemical and functional properties of the different by-products of artichoke $(\mathrm{Cy}$ nara scolymus L.) from industrial canning processing. Food Chem. 160: $134-140$

Saavedra, M.J., Aires, A., Dias, C., Almeida, J.A., de Vasconcelos, M.C.B.M., Santos, P., and Rosa, E.A. (2015). Evaluation of the potential of squash pumpkin by-products (seeds and shell) as sources of antioxidant and bioactive compounds. J. Food Sci. Technol. 52: 1008-1015.

Saija, A., Tomaino, A., Lo Cascio, R., Trombetta, D., Proteggente, A., De Pasquale, A., Uccella, N., and Bonina, F. (1999). Ferulic and caffeic acids as potential protective agents against photooxidative skin damage. J. Sci. Food Agriculture 79: 476-480.

Saito, M., Hosoyama, H., Ariga, T., Kataoka, S., and Yamaji, N. (1998). Antiulcer activity of grape seed extract and procyanidins. J. Agric. Food Chem. 46: 1460-1464.

Sakanaka, S. (1991). Prevention Effects of Tea Polyphenols Against Dental Caries. Proc. of Intern. Symp. on Tea Science, pp. 243-247, 26-29 August, Shizuoka, Japan.

Sakanaka, S. (1995). Anti-caries and Anti-periodontal Disease Effects of Green Tea Polyphenols. Proc. of Intern. Symp. on Tea Quality-Human Health, pp. 97-106, 7-10 December 1995, Shanghai, China.

Sakanaka, S., Shimura, N., Aizawa, M., Kim, M., and Yamamoto, T. (1992). Preventive effect of green tea polyphenols against dental caries in conventional rats. Biosci. Biotechnol. Biochem. 56: 592-594.

Salah, N., Miller, N.J., Parganga, G., Tifburg, L., Bolwell, G.P., and ice-Evan, C. (1995). Polyphenolic flavonols as scavengers of aqueous phase radicals and as chain-breaking antioxidants. Arch. Biochem. Biophys. 322: 339-346.

Salinas-Moreno, Y., Rojas-Herrera, L., Sosa Montes, E., and Pérez-Herrera, P. (2005). Anthocyanin composition in black bean (Phaseolus vulgaris L.) varieties grown in Mexico. Agrocienc. 39: 385-394.

Samaranayake, A.G.P., John, J.A., and Shahidi, F. (2008). Antioxidant activity of English walnut (Juglans Regia L.). J. Food Lipids. 15: 384-397.

Samman, S., Lyons, W.P.M., and Cook, N.C. (1998). Flavonoids and coronary heart disease: Dietary perspectives. Flavonoids in health and disease. In: Rice-Evans, C.A., and Packer, L. (Ed.). Marcel Dekker, New York, NY, pp. 469-482.

Sanchez, G.M., Re, L., Giuliani, A., Nunez-Selles, A., Davison, G.P., and Leon-Hernandez, O.S. (2000). Protective effects of Mangifera indica L. extract mangiferin and selected antioxidants against TPA-induced biomolecules oxidation and peritoneal macrophage activation in mice. Pharmacol. Res. 42: 565-573.

Sánchez-Patán, F., Cueva, C., Monagas, M., Walton, G.E., Gibson, M., G, R. Quintanilla-López, J.S.E., Lebrón-Aguilar, R., Martin-Alvarez, P., Moreno-Arribas, M.V., and Bartolomé, B.A. (2012). In vitro fermentation of a red wine extract by human gut microbiota: changes in microbial groups and formation of phenolic metabolites. J. Agric. Food Chem. 60(9): 2136-2147.

Sanchez-Rabaneda, F., Jauregui, O., Lamuela-Raventos, R.M., Bastida, J. Viladomat, F., and Codina, C. (2003). Identification of phenolic compounds in artichoke waste by high-performance liquid chromatography-tandem mass spectrometry. J. Chromatogr. A 1008: 57-72.

Sang, S., Lapsley, K., Jeong, W.S., Lachance, P.A., Ho, C.T., and Rosen, R.T. (2002b). Antioxidant phenolic compounds isolated from almond skins (Prunus amygdalus Batsch). J. Agric. Food Chem. 50: 24592463.

Sang, S., Lapsley, K., Rosen, R.T., and Ho, C.T. (2002a). New prenylated benzoic acid and other constituents from almond hulls (Prunus amygdalus Batsch). J. Agric. Food Chem. 50: 607-609.

Santos-Buelga, C., Francia-Aricha, E.M., and Escribano-Bailon, M.T. (1995). Comparative flavon-3-ol composition of seeds from different grape varieties. Food Chem. 53: 197-201.

Sato, M., Pei, R.-J., Yuri, Y., Danbara, N., Nakane, Y., and Tsubura, A. (2003) Prepubertal resveratrol exposure accelerates $N$-methyl- $N$-nitrosoureainduced mammary carcinoma in female Sprague-Dawley rats.
Cancer Lett. 202: 137-145.

Saulnier, L., Marot, C., Chanliaud, E., and Thibault, J.E. (1995). Cell wall polysaccharide interaction in maize bran. Carbohydr. Polym. 26: 279-287.

Saura-Calixto, F. (1998). Antioxidant dietary fibre product: a new concept and a potential food ingredient. J. Agric. Food Chem. 46: 4303-4306.

Scalbert, A., and Williamson, G. (2000). Dietary intake and bioavailability of polyphenols. J. Nutr. 130: 2073S-2085S

Scalbert, A., Morand, C., Manach, C., and Remesy, C. (2002). Absorption and metabolism of polyphenols in the gut and impact on health. $\mathrm{Bi}-$ omed. Pharmacother. 56: 276-282.

Schieber, A., Berardini, N., and Carle, R. (2003b). Identification of flavonol and xanthol glycosides from mango peels by HPLC. J. Agric. Food Chem. 51: 5006-5011.

Schieber, A., Hilt, P., Streker, P., Endreß, H.U., Rentschler, C., and Carle, R. (2003a). A new process for the combined recovery of pectin and phenolic compounds from apple pomace. Innovat. Food Sci. Emerg. Tech. 4: 99-107.

Schieber, A., Keller, P., and Carle, R. (2001a). Determination of phenolic acids and flavonoids of apple and pear by high-performance liquid chromatography. J. Chromatogr. A 910: 265-273.

Schieber, A., Stintzing, F.C., and Carle, R. (2001b). By-products of plant food processing as a source of functional compounds-recent developments. Trends Food Sci. Technol. 12: 401-413.

Schmidtlein, H., and Herrmann, K. (1975). Quantitative analysis for phenolic acids by thin-layer chromatography. J. Chromatogr. 115: 123127.

Seeram, N.P., Aronson, W.J., Zhang, Y., Henning, S.M., Moro, A., Lee, R.P., Sartippour, M., Harris, D.M., Rettig, M., Suchard, M.A., Pantuck, A.J., Belldegrun, A., and Heber, D. (2007). Pomegranate ellagitannin-derived metabolites inhibit prostate cancer growth and localise to the mouse prostate gland. J. Agric. Food Chem. 55: 7732-7737.

Seeram, N.P., Aviram, M., Zhang, Y., Henning, S.M., Feng, L., Dreher, M., and Heber, D. (2008). Comparison of antioxidant potency of commonly consumed polyphenol-rich beverages in the United States. J. Agric. Food Chem 56: 1415-1422.

Seeram, N.P., Henning, S.M., Zhang, Y., Suchard, M., Li, Z., and Heber, D. (2006). Pomegranate juice ellagitannin metabolites are present in human plasma and some persist in urine for up to 48 hours. J. Nutr. 136: 2481-2485

Seeram, N., Lee, R., Hardy, M., and Heber, D. (2005). Rapid large scale purification of ellagitannins from pomegranate husk, a by-product of the commercial juice industry. Sep. Pur. Technol. 41: 49-55.

Sekhon-Loodu, S., Warnakulasuriya, S.N., Rupasinghe, H.P.V., and Shahidi, F. (2013). Antioxidant ability of fractionated apple peel phenolics to inhibit fish oil oxidation. Food Chem. 140: 189-196.

Senevirathne, M., Jeon, Y.Y., Ha, J.H., and Kim, S.H. (2009). Effective drying of citrus by-product by high speed drying: A novel drying technique and their antioxidant activity. J. Food Eng. 92: 157-163.

Serrano, J., Puupponen-Pimiä, R., Dauer, A., Aura, A-M., and Saura-Calixto, F. (2009). Tannins: Current knowledge of food sources, intake, bioavailability and biological effects. Mol. Nutr. Food Res. 53: S310-S329.

Servili, M., Baldioli, M., Selvaggini, R., Miniati, E., Macchioni, A., and Montedoro, G. (1999). High-performance liquid chromatography evaluation of phenols in olive fruit, virgin olive oil, vegetation waters, and pmace and 1D-and 2D-nuclear magnetic resonance characterization. J. Am. Oil Chem. Soc. 76: 873-882.

Sesink, A.L.A., O'Leary, K.A., and Hollman, P.C.H. (2001). Quercetin glucuronides but not glucosides are present in human plasma after consumption of quercetin-3-glucoside or quercetin-4'-glucoside. J. Nutr. 131: 1938-1941.

Setchell, K.D.R., and Adlercreutz, H. (1988). Mammalian lignans and phyto-estrogens: Recent studies on their formation, metabolism and biological role in health and disease. Role of the Gut Flora in Toxicity and Cancer. In: Rowland, I. (Ed.). Academic Press, London, UK, pp. 315-345.

Setchell, K.D., Brown, N.M., and Lydeking-Olsen, E. (2002). The clinical importance of the metabolite equol-a clue to the effectiveness of soy and its isoflavones. J. Nutr. 132(12): 3577-3584.

Setianto, W.B., Yoshikawa, S., Smith Jr, R.L., Inomata, H., Florusse, L.J., and Peters, C.J. (2008). Pressure profile separation of phenolic liq- 
uid compounds from cashew (Anacardium occidentale) shell with supercritical carbon dioxide and aspects of its phase equilibria. The J. Supercritic. Fluids 48: 203-210.

Sfahlan, A.J., Mahmoodzadeh, A., Hasanzadeh, A., Heidari, R., and Jamei, R. (2009). Antioxidants and antiradicals in almond hull and shell (Amygdalus communis L.) as a function of genotype. Food Chem. 115: 529-533.

Shahidi, F. (1997). Natural antioxidants: an overview. Natural antioxidants: Chemistry, health effects and applications. In: Shahidi, F. (Ed.). AOCS Press, Champaign, IL, pp. 1-7.

Shahidi, F. (2000). Antioxidant in food and food antioxidants. Nahrung 44: 158-163.

Shahidi, F. (2009). Nutraceuticals and functional foods: Whole versus processed foods. Trends Food Sci.Technol. 20: 376-387.

Shahidi, F., Alasalvar, C., and Liyana-Pathirana, C. (2007). Antioxidant phytochemicals in hazelnut kernel and hazelnut by-products. J. Agric. Food Chem. 55: 1212-1220.

Shahidi, F., and Ambigaipalan, P. (2015). Phenolics and polyphenolics in foods, beverages and spices: Antioxidant activity and health effectsA review. J. Funct. Foods 18: 820-897

Shahidi, F., and Chandrasekara, A. (2013). Millet grain phenolics and their role in disease risk reduction and health promotion: A review. J. Funct. Foods 5: 570-581.

Shahidi, F., Chavan, U.D., Naczk, M., and Amarowicz, R. (2001). Nutrient distribution and phenolic antioxidants in air-classified fractions of beach pea (Lathyrus maritimus L.). J. Agric. Food Chem. 49: 926-933.

Shahidi, F., and Peng, H. (2018). Bioaccessibility and bioavailability of phenolic compounds. J. Food Bioact. 4: 11-68.

Shahidi, F., and Ho, C.-T. (2007). Antioxidant measurement and applications. ACS Symposium Series 956. American Chemical Society, Washington DC.

Shahidi, F., Bagchi, D., and Losso, J.N. (2007). Anti-angiogenic functional and medicinal foods. CRC Press.

Shahidi, F., and Naczk, M. (2003). Phenolics in food and nutraceuticals CRC Press,

Shahidi, F., and Naczk, M. (1995). Food phenolics: Sources, chemistry, effects, applications. Technomic Publishing Company Inc, Lancaster PA

Shahidi, F., Liyana-Pathirana, C.M., and Wall, D.S. (2006). Antioxidant activity of white and black sesame seeds and their hull fraction. Food Chem. 99: 478-483.

Shahidi, F., and Naczk, M. (1995). Food phenolics: Sources, chemistry, effects, applications Technomic Publishing Company Inc, Lancaster PA

Shahidi, F., and Naczk, M. (2004). Phenolics in food and nutraceuticals: Sources, applications and health effects CRC Press, Boca Raton, FL, 131-155.

Shahidi, F., and Wanasundara, P.K.J.P.D. (1992). Phenolic antioxidants. Crit. Rev. Food Sci. Nutr. 32: 67-103.

Shahidi, F., and Yeo, J.-D. (2016). Insoluble-bound phenolics in food. Molecules 21: 1216

Shahidi, F., and Yeo, J.D. (2018). Bioactivities of phenolics by focusing on suppression of chronic diseases: A review. Int. J. Mol. Sci. 19: 1573

Shahidi, F., Zhong, Y., Wijeratne, S.S.K., and Ho, C.T. (2009). Almon and Almond Products: Nutraceutical Components and Health Effects. Tree nuts: Composition, Phytochemicals, and Health effects. In: Alasalvar, C., and Shahidi, F. (Ed.). CRC press, Boca Raton, FL, pp. 127-138.

Shahrzad, S., and Bitsch, I. (1998). Determination of gallic acid and its metabolites in human plasma and urine by high-performance liquid chromatography. J. Chromatogr B. 705: 87-95.

Sharma, H.S.S. (1989). Thermogravimetric analysis of flax shives degraded by Pleurotus ostreatus and Ceraceomyces sublae Vis. Thermochim. Acta 128: 347-357.

Sharma, S.K., and Le Maguer, M. (1996). Lycopene in tomatoes and tomato pulp fractions. Ital. J. Food Sci. 2: 107-113.

Shi, J., and LeMaguer, M. (2000). Lycopene in tomatoes: Chemical and physical properties affected by food processing. Crit. Rev. Food Sci. Nutr. 40: 1-42.

Shi, J., Yu, J., Pohorly, J.E., and Kakuda, Y. (2003). Phenolic in grape seedsBiochemistry and functionality. J. Med. Food 6: 291-299.

Shi, Y., Jia, M., Xu, L., Fang, Z., Wu, W., Zhang, Q., Chung, P., Lin, Y., Wang, S., and Zhang, Y. (2019). miR-96 and autophagy are involved in the beneficial effect of grape seed proanthocyanidins against high fat diet induced dyslipidemia in mice. Phytother. Res. In press.

Shi, Y., Kamer, P.C.J., and Cole-Hamilton, D.J. (2019). Synthesis of pharmaceutical drugs from cardanol derived from cashew nut shell liquid. Green Chem. 21: 1043-1053.

Shin, P.K., Zoh, Y., Choi, J., Kim, M.S., Kim, Y., and Choi, S.W. (2019). Walnut phenolic extracts reduce telomere length and telomerase activity in a colon cancer stem cell model. Nutr. Res. Pract. 13(1): 58-63.

Shixian, Q., Dai, Y., Kakuda, Y., Shi, J., Mittal, G., Yeung, D., and Jiang, Y. (2005). Synergistic Anti-Oxidative Effects of Lycopene with Other Bioactive Compounds. Food Rev. Int. 21: 295-311.

Shobha, S.V., Ramdoss, C.S., and Ravindranath, B. (1994). Inhibition of soybean lipoxygenase- 1 byanacardic acids, cardols, and vardanols. J. Nat. Prod. 57: 1755-1757.

Simmonds, N.W. (1962). Anthocyanins in wild bananas. The evolution of bananas. Longmans, London, UK, pp. 23-25.

Sims, C.A., Balaban, M.O., and Matthews, R.F. (1993). Optimization of carrot juice color and cloud stability. J. Food Sci. 58: 1129-1131.

Singh, B.N., Singh, B.R., Singh, R.L., Prakash, D., Singh, D.P., Sarma, B.K., Upadhyay, G., and Singh, H.B. (2009). Polyphenolics from various extracts/fractions of red onion (Allium cepa) peel with potential antioxidant and antimutagenic activities. Food Chem. Toxicol. 47: 1161-1167.

Singh, J., Upadhyay, A.K., Bahadur, A., Singh, B., Singh, K.P., and Rai, M (2006). Antioxidant phytochemicals in cabbage (Brassica oleracae L. var. capitata). Sci Hortic Amsterdam 108: 233-237.

Singh, M., Arseneault, M., Sanderson, T., Morthy, V., and Ramassamy, C. (2008). Challenges for research on polyphenols from foods in Alzeheirmer's disease: Bioavailability, metabolism and cellular and moleculer mechanism. J. Agric. Food Chem. 56: 4855-4873.

Singh, N., and Rajini, P.S. (2008). Antioxidant-mediated protective effect of potato peel extract in erythrocytes against oxidative damage. Chemico-Biol. Inter. 173: 97-104.

Singh, R.P., Chidambara Murthy, K.N., and Jayaprakasha, G.K. (2002). Studies on the antioxidant activity of pomegranate (Punica granatum) peel and seed extracts using in vitro models. J. Agric. Food Chem. 50: 81-86.

Siriwardhana, S.S.K.W., and Shahidi, F. (2002). Antiradical activity of extracts of almond and its by-products. Am. J. Oil Chem. Soc. 79: 903908.

Slatnar, A., Mikulic-Petkovsek, M., Stampar, F., Veberic, R., and Solar, A. (2015). Identification and quantification of phenolic compounds in kernels, oil and bagasse pellets of common walnut (Juglans regia L.) Food Res. Int. 67: 255-263.

Soares, D.G., Andreazza, A.C., and Salvador, M. (2003). Sequestering ability of butylated hydroxytoluene, propyl gallate, resveratrol, and vitamins $C$ and $E$ against ABTS, DPPH, and hydroxyl free radicals in chemical and biological systems. J. Agric. Food Chem. 51: 1077-1080.

Soleas, G.J., Diamandis, E.P., and Goldberg, D.M. (1997). Resveratrol: A molecule whose time has come? And gone? Clin. Biochem. 30: 91113.

Soong, Y.Y., and Barlow, P.J. (2004). Antioxidant activity and phenolic content of selected fruit seeds. Food Chem. 88: 411-417.

Soong, Y.Y., and Barlow, P.J. (2005). Isolation and structure elucidation of phenolic compounds from longan (Dimocarpus longan Lour.) seed by high-performance liquid chromatography-electrospray ionization mass spectrometry. J. Chromatogr. A 1085: 270-277.

Soong, Y.Y., and Barlow, P.J. (2006). Quantification of gallic acid and ellagic acid from longan (Dimocarpus longan Lour.) seed and mango (Mangifera indica L.) kernel and their effects on antioxidant activity. Food Chem. 97: 524-530.

Soong, Y.Y., Barlow, P.J., and Perera, C.O. (2004). A cocktail of phytonutrients: identification of polyphenols, phytosterols and tocopherols from mango (Mangifera indica L.) seed kernel. IFT annual meeting, Las Vegas.

Souquet, J.M., Cheynier, V., and Moutounet, M. (2000). Les proantocyanidines du raisin, Bulletin of the OIV 835-836, pp. 601-609.

Souquet, J.M., Cheynier, V., Brossaud, F., and Moutounet, M. (1996). Polymeric proanthocyanidins from grape skins. Phytochemistry 43 : 509-512.

Ssonko, U.L., and Xia, W. (2005). Food phenolics, pros and cons: review. 
Food Rev. Int. 21: 367-388

Stafford, H.A. (1994). Review article. Anthocyanins and betalains: evolution of the mutually exclusive pathways. Plant Science 101: 91-98.

Stewart, A.J., Bozonnet, S., Mullen, W., Jenkins, G.I., Lean, M.E.J., and Crozier, A. (2000). Occurrence of flavonols in tomatoes and tomatobased products. J. Agric. Food Chem. 48: 2663-2669.

Stintzing, F.C., and Carle, R. (2004). Functional properties of anthocyanins and betalains in plants, food and in human nutrition. Trends Food Sci. Technol. 15: 19-38.

Stintzing, F.C., Schieber, A., and Carle, R. (2001). Phytochemical and nutritional significance of cactus pear. Eur. Food Res. Technol. 212: 396-407.

Stoll, T., Schieber, A., and Carle, R. (2001). Carrot pomace-an underestimated by-product? Biologically-active phytochemicals in food: analysis, metabolism, bioavailability and function. In: Pfannhauser, W.Fenwick, G.R., and Khokhar, S. (Ed.). Royal Society of Chemistry, Cambridge, UK, pp. 525-527.

Stoner, G.D., and Mukhtar, H. (1995). Polyphenols as cancer chemopreventive agents. J. Cellular Biochem. 22: 169-180.

Su, M.S., and Silva, J.L. (2006). Antioxidant activity, anthocyanins, and phenolics of rabbiteye blueberry (Vaccinium ashei) by-products as affected by fermentation. Food Chem. 97: 447-451.

Suarez-Valles, B., Santamaria-Victorero, J., Mangas-Alonso, J.J., and Blanco-Gomis, D. (1994). High-performance liquid chromatography of the neutral phenolic compounds of low molecular weight in apple juice. J. Agric. Food Chem. 42: 2732-2736.

Subbaramaiah, K., Chung, W.J., Michaluart, P., Telang, N., Tanabe, T., Inoue, H., Jang, M., Pezzuto, J.M., and Dannenberg, A.J. (1998). Resveratrol inhibits cyclooxygenase-2 transcription and activity in phorbol ester-treated human mammary epithelial cells. J. Biol. Chem. 273: 21875-21882.

Subhashinee, S.S.K.W., Amarowicz, R., and Shahidi, F. (2006). Antioxidant activity of almonds and their by-products in food model systems. J. Am. Oil Chem. Soc. 83: 223-230.

Sudha, M.L., Baskaran, V., and Leelavathi, K. (2007). Apple pomace as a source of dietary fiber and polyphenols and its effect of the rheological characteristics and cake making. Food Chem. 104: 686-692.

Suja, K.P., Jayalekshmy, A., and Arumughan, C. (2005). Antioxidant activity of sesame cake extract. Food Chem. 91: 213-219.

Sultana, B., Anwar, F., and Przybylski, R. (2007). Antioxidant potential of corncob extracts for stabilization of corn oil subjected to microwave heating. Food Chem. 104: 997-1005.

Sultana, B., Anwar, F., Asi, M.R., and Chatha, S.A.S. (2008). Antioxidant potential of extracts from different agro wastes: Stabilization of corn oil. Grasas aceites 59: 205-217.

Sumner, M.D., Elliott-Eller, M., Weidner, G., Daubenmier, J.J., Chew, M.H., Marlin, R., Raisin, C.J., and Ornish, D. (2005). Effects of pomegranate juice consumption on myocardial perfusion in patients with coronary heart disease. Am. J. Cardiol. 96: 810-814.

Sun, J., Shi, J., Jiang, Y., Xue, S.J., and Wei, X. (2007). Identification of two polyphenolic compounds with antioxidant activities in longan pericarp tissues. J. Agric. Food Chem. 55: 5864-5868.

Sun, R.C., Sun, X.F., Wang, S.Q., Zhu, W., and Wang, X.Y. (2002). Ester and ether linkages between hydroxycinnamic acids and lignins from wheat, rice, rye, and barley straws, maize stems, and fast-growing poplar wood. Ind. Crops Prod. 15: 179-188.

Sun, Y., Tsao, R., Chen, F., Li, H., Wang, J., Peng, H., Zhang, K., and Deng, Z (2017). The phytochemical composition, metabolites, bioavailability and in vivo antioxidant activity of Tetrastigma hemsleyanum leaves in rats. J. Funct. Foods 30: 179-193.

Sun, Y., Zhou, D., and Shahidi, F. (2018). Antioxidant properties of tyrosol and hydroxytyrosol saturated fatty acid esters. Food Chem. 245 1262-1268.

Swanston-Flatt, S.K.D.A.Y., C. Bailey, C.J., and Flatt, P.R. (1989). Evaluation of traditional plant treatments for diabetes: Studies in streptozotocin diabetic mice. Acta Diabetol Lat 26: 51-55

Sze-Tao, K.W.C., and Sathe, S.K. (2000). Walnut (Juglans regia L.): Proximate composition, protein solubility, protein amino acid composition and protein in vitro digestability. J. Sci. Food Agric. 80: 1393-1401.

Takahashi, T., Kamimura, A., Shirai, A., and Yokoo, Y. (2000). Several Selective Protein Kinase C Inhibitors Including Procyanidins Promote Hair
Growth, Skin. Pharmaco. Appl. Skin Physiol. 13: 133-142.

Takahashi, T., Kamiya, T., Hasegawa, A., and Yokoo, Y. (1999). Procyanidins oligomers selectively and intensively promote proliferation of mouse hair epithelial cells in vitro and activate hair follicle growth in vivo. J. Investig. Dermatol. 112: 310-316.

Takahata, Y., Ohnishi-Kameyana, M., Furuta, S., Takahasi, M., and Suda, I. (2001). Highly polymerized procyanidins in brown soybean seed coat with a high radical-scavenging activity. J. Agric. Food Chem. 49: 5743-5747.

Takaya, Y., Yan, K.X., Terashima, K., He, Y.H., and Niwa, M. (2002). Biogenetic reactions of stilbenetetramers from Vitaceous plants. Tetrahedron 58: 9265-9271.

Takeoka, G.R., Dão, L.T., Full, G.H., Wong, R.Y., Harden, L.A., Edwards, R.H., and Berrios, J.D.J. (1997). Characterization of black bean (Phaseolus vulgaris L.) anthocyanins. J. Agric. Food Chem. 45: 3395-3400.

Takeoka, G., Dao, L., Teranishi, R., Wong, R., Flessa, S., Harden, L., and Edwards, R. (2000). Identification of three triterpenoids in almond hulls. J. Agric. Food Chem. 48: 3437-3439.

Tanaka, T., Makita, H., Kawabata, K., Mori, H., Kakumoto, M., Satoh, K., Hara, A., and Sumida, T. (1997). Chemoprevention of azoxymethaneinduced rat colon carcinogenesis by the naturally occurring flavonoids, diosmin and hesperidin. Carcinogenesis 18: 957-965.

Tanizawa, H., Toda, S., Sazuka, Y., Taniyama, T., Hayashi, T., Arichi, S., and Takino, Y. (1994). Natural antioxidants. I. Antioxidative components of tea leaf (Thea sinensis L.). Chem. Pharm. Bull. 32: 2011-2014.

Taylor, A., Jacques, P.F., and Epstein, E.M. (1995). Relationship among aging, antioxidant status and cataract. Am. J. Clin. Nutr. 62: 1439S1447S.

Tebib, K., Bitri, L., Besanncon, P., and Rouanet, J.-M. (1994). Polymeric grape seed tannins prevent plasma cholesterol changes in high-cholesterol-fed rats. Food Chem. 49: 403-406.

Teh, S.-S., Niven, B.E., Bekhit, A.E.-D.A., Carne, A., and Birch, E.J. (2015). Microwave and pulsed electric field assisted extractions of polyphenols from defatted canola seed cake. Int. J. Food Sci. Technol. 50: 1109-1115.

Teissedre, P.L., Frankel, E.N., Waterhouse, A.L., Peleg, H., and German, J.B. (1996). Inhibition of in vitro human LDL oxidation by phenolic antioxidants from grapes and wines. J. Sci. Food Agric. 70: 55-61.

Terpinc, P., Ceh, B., Ulrih, N.A.P., and Abramoviç, H. (2012). Studies of the correlation between antioxidant properties and the total phenolic content of different oil cake extracts. Ind. Crop. Prod. 39: 210-217.

Terra, X., Pallarés, V., Ardèvol, A., Bladé, C., Fernández-Larrea, J., Pujadas, G., Salvadó, J., Arola, L., and Blay, M. (2011). Modulatory effect of grape-seed procyanidins on local and systemic inflammation in dietinduced obesity rats. J. Nutr. Biochem. 22(4): 380-387.

Tessitore, L., Davit, A., Sarotto, I., and Caderni, G. (2000). Resveratrol de presses the growth of colorectal aberrant crypt foci by affecting bax and p21 (CIP) expression. Carcinogenesis 21: 1619-1622.

Thanaraj, S.N.S., and Seshadri, R. (1990). Influence of polyphenol oxidase activity and polyphenol content of tea shoot on the quality of black tea. J. Sci. Food Agric. 51: 57-69.

Thompson, L.U. (1993). Potential health benefits and problems associated with antinutrients in foods. Food Res. Intnl. 26: 131-149.

Thompson, L.U., Rickard, S.E., Orcheson, L.J., and Fong, H.H.S. (1996a). Flaxseed and its lignan and oil components reduce mammary tumor growth at a late stage of carcinogenesis. Carcinogenesis 17: 13731376 .

Thompson, L.U., Seidl, M.M., Rickard, S., Orcheson, L., and Fong, H.H.S (1996b). Antitumorigenic effect of a mammalian lignan precurso from flaxseed. Nutr. Cancer 26: 159-165.

Tijburg, L.B.M., Mattern, T., Folts, J.D., Weisgerber, U.M., and Katan, M.B. (1997). Tea flavonoids and cardiovascular diseases: A review. Crit. Rev. Food Sci. Nutr. 37: 771-785.

Tomás-Barberán, F.A., and Clifford, M.N. (2000). Dietary hydroxybenzoic acid derivatives-nature, occurrence and dietary burden. J. Sci. Food Agric. 80: 1024-1032.

Tomás-Barberán, F.A., Llorach, R., Espín, J.C., and Ferreres, F. (2004). Agrifood residues as a source of phytochemicals. Total Food. In: Keith, W.Craig, F., and Andrew, S. (Ed.). Institute of Food Research, Proceedings, pp. 42-48.

Toomer, O.T. (2018). A comprehensive review of the value-added uses of 
peanut (Arachis hypogaea) skins and by-products. Crit. Rev. Food Sci. Nutr. 1-10.

Toor, R.K., and Savage, G.P. (2005). Antioxidant activity in different fractions of tomatoes. Food Res. Int. 38: 487-494.

Toyomizu, M., Okamoto, K., Ishibashi, T., Chen, Z., and Nakatsu, T. (1999). Uncoupling effect of anacardic acids from cashew nut shell oil on oxidative phosphorylation of rat liver mitochondria. Life Sci. 66: 229-234.

Trevisan, M.T.S., Pfundstein, B., Haubner, R., Würtele, G., Spiegelhalder, B. Bartsch, H., and Owen, R.W. (2006). Characterization of alkyl phenols in cashew (Anacardium occidentale) products and assay of their antioxidant capacity. Food Chem. Toxicol. 44: 188-197.

Tripoli, E., Guardia, M.L., Giammanco, S., Majo, D.D., and Giammanco, M. (2007). Citrus flavonoids: molecular structure, biological activity and nutritional properties: A review. Food Chem. 104: 466-479.

Troszynska, A., and Ciska, E. (2002). Phenolic compounds of seed coats of white and coloured varieties of pea (Pisum sativum L.) and their total antioxidant activity. Czech. J. Food Sci. 20: 15-22.

Tsuda, T., Horio, F., and Osawa, T. (1999). Absorption and metabolism of cyanidin 3-O-B-D-glucoside in rats. FEBS Lett. 449: 179-182.

Türk, G., Sönmez, M., Aydin, M., Yüce, A., Gür, S., Yüksel, M., Aksu, E., and Aksoy, H. (2008). Effects of pomegranate juice consumption on sperm quality, spermatogenic cell density, antioxidant activity and testosterone level in male rats. Clin. Nutr. 27: 289-296.

Tyagi, A., Kumar, S., Raina, K., Wempe, M.F., Maroni, P.D., Agarwal, R., and Agarwal, C. (2019). Differential effect of grape seed extract and its active constituent procyanidin B2 3, 3"-di-O-gallate against prostate cancer stem cells. Mol. Carcinog.

Tyman, J.H.P., Tychopoulos, V., and Chan, P. (1984). Long-chain phenols: XXV. Quantitative analysis of natural cashew nut-shell liquid (Anacardium occidentale) by high-performance liquid chromatography. J. Chromatogr. 303: 137-150.

Tyug, T.S., Johar, M.H., and Ismail, A. (2010). Antioxidant properties of fresh, powder, and fiber products of mango (Mangifera foetida) fruit. Intern.I J. Food Proper. 13: 682-691.

Tzulker, R., Glazer, I., Bar-llan, I., Holland, D., Aviram, M., and Amir, R. (2007). Antioxidant activity, polyphenol content, and related compounds in different fruit juices and homogenates prepared from 29 different pomegranate accessions. J. Agric. Food Chem. 55: 95599570.

Ueda, M., Sasaki, K.S., Utsunimiya, N., Inaba, K., and Bayashi, Y.S. (2000) Variation of total polyphenol and polyphenol oxidase activity during maturation of mango fruit (Mangifera Indica L. Irwin) cultured in plastic green house. Food Sci. Tech. Res. 6: 299-305.

Ulrich, D., Hoberg, E., Bittner, T., Engewald, W., and Meilchen, K. (2001) Contribution of voaltile compounds to the flavor of cooked asparagus. Eur. Food Res. Technol. A 213: 200-204.

Vahabzadeh, F., Mehranian, M., and Mofarrah, E. (2004). Antioxidant activity of pistachio hulls. J. Am. Oil Chem. Soc. 81: 621-622.

Valdés, A., Vidal, L., Beltran, A., Canals, A., and Garrigos, M.C. (2015). Microwave-assisted extraction of phenolic compounds from almond skin byproducts (Prunus amygdalus): A multivariate analysis approach. J. Agric. Food Chem. 63: 5395-5402.

van der Sluis, A.A., Dekker, M., de Jager, A., and Jongen, W.M.F. (2001). Activity and concentration of polyphenolic antioxidants in apple: effect of cultivar, harvest year, and storage conditions. J. Agric. Food Chem. 49: 3606-3613.

Vazquez-Olivo, G., López-Martínez, L.X., Contreras-Angulo, L., and Heredia, J.B. (2019). Antioxidant capacity of lignin and phenolic compounds from corn stover. Waste Biomass. Valorization 10: 95-102.

Velioglu, Y.S., Mazza, G., Gao, L., and Oomah, B.D. (1998). Antioxidant activity and total phenolics in selected fruits, vegetables, and grain products. J. Agric. Food Chem. 46: 4113-4117.

Verma, B., Hucl, P., and Chibbar, R.N. (2008). Phenolic content and antioxidant properties of bran in 51 wheat cultivars. Cereal Chem. 85 544-549.

Ververidis, F., Trantas, E., Douglas, C., Vollmer, G., Kretzschmar, G., and Panopoulos, N. (2007). Biotechnology of flavonoids and other phenylpropanoid-derived natural products. Part I: Chemical diversity, impacts on plant biology and human health. Biotechnol. J. 2: 12141234.
Vijaya, K., Ananthan, S., and Nalini, R. (1995). Antibacterial effect of theaflavin, polyphenon 60 (Camellia sinensis) and Euphoribia hirta on Shigella spp. - a cell culture study. J. Ethnopharmacol. 49: 115118.

Vinson, A., Dabbagh, Y.A., Serry, M.M., and Jang, J. (1995). Plant flavonoids, especially tea flavonoids, are powerful antioxidants using an in vitro oxidation model for heart disease. J. Agric. Food Chem. 43: 2800-2802

Vinson, J.A., Hao, Y., Su, X., and Zubik, L. (1998). Phenol antioxidant quantity and quality in foods: Vegetables. J. Agric. Food Chem. 46: 36303634.

Nour, V., Ionica, M.E., and Trandafir, I. (2015). Bread enriched in lycopene and other bioactive compounds by addition of dry tomato waste. J. Food Sci. Technol. 52: 8260-8267.

Visioli, F., and Galli, C. (2003). Olives and their production waste products as sources of bioactive compounds. Curr. Topics Nutr. Res. 1: 85-88.

Visioli, F., Bellosta, S., and Galli, C. (1998). Oleuropein, the bitter principle of olives, enhances nitric oxide production by mouse macrophages. Science 62: 541-546.

Visioli, F., Galli, C., Bornet, F., Mattei, A., Patelli, R., Galli, G., and Caruso, D. (2000). Olive oil phenolics are dose-dependently absorbed in humans. FEBS Lett. 468: 159-60.

Visioli, F., Galli, C., Grande, S., Colonnelli, K., Patelli, C., Galli, G., and Caruso, D. (2003). Hydroxytyrosol excretion differs between rats and humans and depends on the vehicle of administration. J. Nutr. 133 2612-2615.

Vissers, M.H., Zock, P.L., Roodenburg, A.J., Leenen, R., and Katan, M.B. (2002). Olive oil phenols are absorbed in humans. J. Nutr. 132: 409417.

Vitaglione, P., Sforza, S., Galaverna, G., Ghidini, C., Caporaso, N., Vescovi, P.P., Fogliano, V., and Marchelli, R. (2005). Bioavailability of trans-resveratrol from red wine in humans. Mol. Nutr. Food Res. 49: 495-504.

Vrhosek, U., Rigo, A., Tonan, D., and Mattivi, F. (2004). Quantitation of polyphenols in different apple varieties. J. Agric. Food Chem. 52: 6532-6538.

Vu, D.C., Vo, P.H., Coggeshall, M.V., and Lin, C.H. (2018). Identification and characterization of phenolic compounds in black walnut kernels. J. Agric. Food Chem. 66(17): 4503-4511.

Vu, H.T., Scarlett, C.J., and Vuong, Q.V. (2019). Maximising recovery of phenolic compounds and antioxidant properties from banana peel using microwave assisted extraction and water. J. Food Sci. Technol. 56: $1360-1370$

Vulić, J.J., Ćebović, T.N., Canadanović-Brunet, J.M., Ćetković, G.S., Ćanadanović, V.M., Djilasa, S.M., and Šaponjac, V.T.T. (2014). In vivo and in vitro antioxidant effects of beetroot pomace extracts. J. Funct. Food 6: 168-175.

Wacker, W.E.C., Margoshes, M., Bartholomay, A.F., and Vallee, B.L. (1958) Banana as a low sodium dietary staple. N. Engl. J. Med. 259: 901.

Waldron, K. (2001). Useful ingredients from onion waste. Food Sci. Technol. 15: 38-39.

Walle, T., Hsieh, F., De Legge, M.H., Oatis, J.E., and Walle, U.K. (2004). High absorption but very low bioavailability of oral resveratrol in humans. Drug. Metab. Dispos. 32: 1377-1382.

Al-Wandawi, H., Abdul-Rahman, M., and Shaikhly, K. (1985). Tomato processing wastes as essential raw material sources. J. Agric. Food Chem. 33: 804-807.

Wang, J., and Mazza, G. (2002). Effects of anthocyanins and other phenolic compounds on the production of tumor necrosis factor alpha in LPS/ IFN-gamma-activated RAW 264.7 macrophages. J. Agric. Food Chem. 50: 4183-4189.

Wang, J., Sun, B., Cao, Y., Tian, Y., and Li, X. (2008). Optimisation of ultrasound-assisted extraction of phenolic compounds from wheat bran. Food Chem. 106: 804-810.

Wang, S.Y., Feng, R., Bowman, L., Penhallegon, R., Ding, M., and Lu, Y (2005). Antioxidant activity in lingonberries (Vaccinium vitis-idaea L.) and its inhibitory effect on activator protein-1, nuclear factor-kap$\mathrm{paB}$, and mitogen-activated protein kinases activation. J. Agric. Food Chem. 53: 3156-3166.

Wang, Z.Y., Agarwal, R., Bickers, D.R., and Mukhtar, H. (1991). Protection against ultraviolet $b$ radiation-induced photocarcinogensis in hairless mice by green tea polyphenols. Carcinogenesis 12: 1527-1530. 
Wang, Z., Huang, Y., Zou, J., Cao, K., Xu, Y., and Wu, J.M. (2002). Effects of red wine and wine polyphenol resveratrol on platelet aggregation in vivo and in vitro. Int. J. Mol. Med. 9: 77-79.

Wanyo, P., Meeso, N., and Sirithon Siriamornpun, S. (2014). Effects of different treatments on the antioxidant properties and phenolic compounds of rice bran and rice husk. Food Chem. 157: 457-463.

Watanabe, M., Ohshita, Y., and Tsushida, T. (1997). Antioxidant compounds from buckwheat (Fagopyrum esculentum Möench) hulls. J. Agric. Food Chem. 45: 1039-1044.

Weichselbaum, E., Wyness, L., and Stanner, S. (2010). Apple polyphenols and cardiovascular disease - a review of the evidence. Nutr. Bulletin 35: 92-101.

Weisz, G.M., Kammerer, D.R., and Carle, R. (2009). Identification and quantification of phenolic compounds from sunflower (Helianthus annuus L.) kernels and shells by HPLC-DAD/ESI-MSn. Food Chem. 115: 758-765.

Wennberg, M., Engqvist, G., and Nyman, E. (2004). Effects of boiling on dietary fibre components in fresh and stored white cabbage (Brassica oleracea var. capitata). J. Food Sci. 68: 1615-1621.

Wenzel, E., and Somoza, V. (2005). Metabolism and bioavailability of trans-resveratrol Review. Mol. Nutr. Food Res. 49: 472-481.

Whale, S.K., and Zora, S. (2007). Endogenous ethylene and color development in the skin of 'Pink Lady' apple. J. Am. Soc. Hortic. Sci. 132: 20-28.

White, P.J., and Xing, Y. (1997). Antioxidants from cereals and legumes. Natural antioxidant, chemistry, health effects and applications. In: Shahidi, F. (Ed.). Champaign, IL, USA, pp. 25-45.

Wijeratne, S.S.K., Abou-Zaid, M.M., and Shahidi, F. (2006). Antioxidant polyphenols in almond and its coproducts. J. Agric. Food Chem. 54: 312-318.

Wijngaard, H.H., Christian, R., and Nigel, B. (2009). A survey of Irish fruit and vegetable waste and by-products as a source of polyphenolic antioxidants. Food Chem. 116: 202-207.

Williams, C.A., and Grayer, R.J. (2004). Anthocyanins and other flavonoids. Nat. Prod. Rep. 21: 539-573.

Williamson, G., and Manach, C. (2005). Bioavailability and bioefficacy of polyphenols in humans. II. Review of 93 intervention studies. Am. J. Clin. Nutr. 81(1): 243S-255S.

Wiseman, S.A., Balentine, D.A., and Frei, B. (1997). Antioxidants in tea. Crit. Rev. Food Sci. Nutr. 37: 705-718.

Woldedecke, M., and Herrmann, K. (1974). Flavanole und flavone der Gemusearten III. Flavanole und flavone der tomaten und des gemusepaprikas. Z. Lebensm. Unters. Forsch. 155: 216-219.

Wolfe, K.L., and Liu, R.H. (2003). Apple peels as a value-added food ingredient. J. Agric. Food Chem. 51: 1676-1683.

Wolfe, K., Xianzhong, W.U., and Liu, R.H. (2003). Antioxidant activity of apple peels. J. Agric. Food Chem. 51: 609-614.

Wrolstad, R.E. (2005). Handbook of food analytical chemistry. Wiley, Hoboken, NJ.

Wu, K., Zhang, W., Addis, P.B., Epley, R.J., Salih, A.M., and Lehrfeld, J. (1994). Antioxidant properties of wild rice. J. Agric. Food Chem. 42: 34-37.

Wu, T., Guan, Y., and Ye, J. (2007). Determination of flavonoids and ascorbic acid in grapefruit peel and juice by capillary electrophoresis with electrochemical detection. Food Chem. 100: 1573-1579.

Wu, X., Cao, G., and Prior, R.L. (2002). Absorption and metabolism of anthocyanins in elderly women after consumption of elderberry or blueberry. J. Nutr. 132: 1856-1871.

Wu, X., Pittman, H.E., McKay, S., and Prior, R.L. (2005). Aglycones and sugar moieties alter anthocyanin absorption and metabolism after berry consumption in weanling pigs. J. Nutr. 135: 2417-2424.

Wyler, H. (1969). Die betalaine. Chemie in unserer Zeit 3: 146-151.

Xing, Y., and White, P. (1997). Identification and function of antioxidants from oat groats and hulls. J. Am. Oil Chem. Soc. 74: 303-307.

Xu, G.H., Chen, J.C., Liu, D.H., Zhang, Y.H., Jiang, P., and Ye, X.Q. (2008) Minerals, phenolic compounds, and antioxidant capacity of citrus peel extract by hot water. J. Food Sci. 73: C11-C18.

Xu, G., Ye, X., Chen, J., and Liu, D. (2007). Effect of heat treatment on the phenolic compounds and antioxidant capacity of citrus peel extract. J. Agric. Food Chem. 55: 330-335.

Xu, H.X., Wan, M., Dong, H., But, P.P.H., and Foo, L.Y. (2000). Inhibitory activity of flavonoids and tannins against HIV-1 protease. Biol. Pharmacol. Bulletin 23: 1072-1076.

Yakhlef, W., Arhab, R., Romero, C., Brenes, M., de Castro, A., and Medina, E. (2018). Phenolic composition and antimicrobial activity of Algerian olive products and by-products. LWT-Food Sci. Tech. 93: 323-328.

Yamakoshi, J., Kataoka, S., Koga, T., and Ariga, T. (1999). Proanthocyanidinrich extract from grape seeds attenates the development of aortic atherosclerosis in cholesterol-fed rabbits. Atherosclerosis 142: 139149.

Yamakoshi, J., Saito, M., Kataoka, S., and Tokutake, S. (2002). Procyanidinrich extract from grape seeds prevents cataract formation in hereditary cataractous (ICR)/f) rats. J. Agric. Food Chem. 50: 4983-4988.

Yamashita, K., lizuka, Y., Imai, T., and Namiki, M. (1995). Sesame seed and its lignans produce marked enhancement of vitamin $E$ activity in rats fed a low-tocopherol diet. Lipids 30: 1019-1028.

Yanagida, A., Kanda, T., Tanabe, M., Matsudaira, F., and Oliveira Cordeiro, J.G. (2000). Inhibitory effects of apple polyphenols and related compounds on cariogenic factors of mutans streptococci. J. Agric. Food Chem. 48: 5666-5671.

Yang, C.S. (1997). Inhibition of carcinogenesis by tea. Nature 389: 134135.

Yang, C.S., Sang, S., Lambert, J.D., and Lee, M.-J. (2008). Bioavailability is sues in studying the health effects of plant polyphenolic compounds. Mol. Nutr. Food Res. 52: S139-S151.

Yang, J., Wu, X.B., Chen, H.L., Sun-waterhouse, D., Zhong, H.B., and Cui, C. (2019). A value-added approach to improve the nutritional quality of soybean meal byproduct: Enhancing its antioxidant activity through fermentation by Bacillus amyloliquefaciens SWJS22. Food Chem. 272: 396-403.

Yasutake, T., Wada, H., Higaki, M., Nakamura, M., Honda, K., Watanabe, M., Ishii, H., Kamiya, S., Takizawa, H., and Goto, H. (2013). Anacardic acid, a histone acetyltransferase inhibitor, modulates LPS-induced IL-8 expression in a human alveolar epithelial cell line A549. F1000Research 2: 78.

Yen, G.C., and Chen, H.Y. (1994). Comparison of antimutagenic effect of various tea extracts (green, oolong, pouchong, and black tea). J. Food Prot. 57: 54-58.

Yen, G.C., and Duh, P.D. (1995). Antioxidant activity of methanolic extracts of peanut hulls from various cultivars. J. Am. Oil Chem. Soc. 72: 1065-1067.

Yen, G.C., Duh, P.D., and Tsai, C.L. (1993). Relationship between antioxidant activity and maturity of peanut hulls. J. Agric. Food Chem. 41: 67-70.

Yen, W.J., Chang, L.W., and Duh, P.N. (2005). Antioxidant activity of peanut seed testa and its antioxidative component, ethyl protocatechuate. Food Sci. Tech. 38: 193-200.

Yeo, J.D., and Shahidi, F. (2017). Effect of hydrothermal processing on changes of insoluble-bound phenolics of lentils. J. Funct. Foods 38 : 716-722.

Yi, C., Shi, J., Kramer, J., Xue, S., Jiang, Y., Zhang, M., Ma, Y., and Pohorly, J. (2009). Fatty acid composition and phenolic antioxidants of winemaking pomace powder. Food Chem. 114: 570-576.

Young, A.J., and Lowe, G.M. (2001). Antioxidant and prooxidant properties of carotenoids. Arch. Biochem. Biophys. 385: 20-27.

Yu, J., Ahmedna, M., and Goktepe, I. (2005). Effects of processing methods and extraction solvents on concentration and antioxidant activity of peanut skin phenolics. Food Chem. 90: 199-206.

Yu, J., Ahmedna, M., Goktepe, I., and Dai, J. (2006). Peanut skin procyanidins: Composition and antioxidant activities as affected by process ing. J. Food Comp. Anal. 19: 364-371.

Yuan, B., Lu, M., Eskridge, K.M., Isom, L.D., and Hanna, M.A. (2018). Extraction, identification, and quantification of antioxidant phenolics from hazelnut (Corylus avellana L.) shells. Food Chem. 244: 7-15.

Zambon, D., Sabate, J., Muñoz, S., Campero, B., Casals, E., Merlos, M., Laguna, J.C., and Ros, E. (2000). Substituting walnuts for monounsaturated fat improves the serum lipid profile of hypercholesterolemic men and women: A randomised crossover trial. Annals Int. Med. 132: 538-546.

Zandi, P., and Gordon, M.H. (1999). Antioxidant activity of extracts from old tea leaves. Food Chem. 64: 285-288.

Zanotti, I., Dall'Asta, M., Mena, P., Mele, L., Bruni, R., Ray, S., and Del Rio, 
D. (2015). Atheroprotective effects of (poly) phenols: a focus on cell cholesterol metabolism. Food Funct. 6(1): 13-31.

Zdunäczyk, Z., Flis, M., Zielinäski, H., Wroäblewska, M., Antoszkiewicz, Z., and Jusäkiewicz, J. (2006). In vitro antioxidant activities of barley, husked oat, naked oat, triticale, and buckwheat wastes and their in fluence on the growth and biomarkers of antioxidant status in rats. J. Agric. Food Chem. 54: 4168-4175.

Zhang, B., Peng, H., Deng, Z., and Tsao, R. (2018). Phytochemicals of lentil (Lens culinaris) and their antioxidant and anti-inflammatory effects. J. Food Bioact. 1: 93-103.

Zhang, D., and Hamauzu, Y. (2004). Phenolic compounds and their antioxidant properties in different tissues of carrots. (Daucus carota L.). Food Agri. Environ. 2: 95-100

Zhao, Z., Egashira, Y., and Sanada, H. (2004). Ferulic acid is quickly absorbed from rat stomach as the free form and then conjugated mainly in liver. J. Nutr. 134: 3083-3088.

Zhao, Z., and Moghadasian, M.H. (2008). Chemistry, natural sources, dietary intake and pharmacokinetic properties of ferulic acid: a review. Food Chem. 109: 691-702.

Zhao, Z., and Moghadasian, M.H. (2009). Bioavailability of hydroxycinnamates: A brief review of in vivo and in vitro studies. Phytochemistry Reviews 9: 133-145.

Zheng, Y., Lee, S.O., Verbruggen, M.A., Murphy, P.A., and Hendrich, S. (2004). The apparent absorptions of isoflavone glucosides and aglucons are similar in women and are increased by rapid gut transit time and low fecal isoflavone degradation. J. Nutr. 134: 2534-2539.

Zhong, J. (2003). Effect of grape procyanidins on proliferation and apoptosis of pancreas beta cells. Endocrine Abstracts 5: 82.

Zhou, D., Sun, Y., and Shahidi, F. (2017). Preparation and antioxidant activity of tyrosol and hydroxytyrosol esters. J. Funct. Foods 37: 66-73.
Zhou, K., and Yu, L. (2004). Antioxidant properties of bran extracts from Trego wheat grown at different locations. J. Agric. Food Chem. 52: 1112-1117.

Zhou, K., Laux, J.J., and Yu, L. (2004a). Comparison of Swiss red wheat grain and fractions for their antioxidant properties. J. Agric. Food Chem. 52: 1118-1123.

Zhou, K., Su, L., and Yu, L. (2004b). Phytochemicals and antioxidant properties in wheat bran. J. Agric. Food Chem. 52: 6108-6114.

Zhou, K., Yin, J., and Yu, L. (2005). Phenolic acid, tocopherol and carotenoid compositions, and antioxidant functions of hard red winter wheat bran. J. Agric. Food Chem. 53: 3916-3922.

Zhou, S., Fang, Z., Lü, Y., Chen, J., Liu, D., and Ye, X. (2009). Phenolics and antioxidant properties of bayberry (Myrica rubra Sieb. et Zucc.) pomace. Food Chem. 112: 394-399.

Zhu, X., Zhang, H., and Lo, R. (2004). Phenolic compounds from the leaf extract of artichoke (Cynara scolymus L.) and their antimicrobial activities. J. Agric. Food Chem. 52: 7272-7278.

Ziegler, C.C., Rainwater, L., Whelan, J., and McEntee, M.F. (2004). Dietary resveratrol does not affect intestinal tumorigenisis in Apc ( $\mathrm{min} /+)$ mice. J. Nutr. 134: 5-10.

Zobel, A.M. (1997). Phytochemistry of Fruit and Vegetables. In: TomásBarberán, F.A., and Robbins, R.J. (Ed.). Clanderon Press, Oxford, UK, pp. 173-204.

Zubik, L., and Meydani, M. (2003). Bioavailability of soybean isoflavones form aglycone and glucoside from in American women. Am. J. Clin. Nutr. 77: 1459-1465.

Zumbrunn, A., Uebelhart, P., and Eugster, C.H. (1985). HPLC of carotenes with end groups and (Z)-configuration at terminal conjugated double bonds, isolation of (5Z)-lycopene from tomatoes. Helv. Chim. Acta. 68: 1540-1542. 